University of Louisville

ThinkIR: The University of Louisville's Institutional Repository

$12-2014$

\title{
Novel analytical tools for studies in molecular assemblies : I. Electro-active single-mode integrated optical waveguides ; II. Coupled plasmon waveguide resonances.
}

Xue Han

University of Louisville

Follow this and additional works at: https://ir.library.louisville.edu/etd

Part of the Physics Commons

\section{Recommended Citation}

Han, Xue, "Novel analytical tools for studies in molecular assemblies : I. Electro-active single-mode integrated optical waveguides ; II. Coupled plasmon waveguide resonances." (2014). Electronic Theses and Dissertations. Paper 1743.

https://doi.org/10.18297/etd/1743

This Doctoral Dissertation is brought to you for free and open access by ThinkIR: The University of Louisville's Institutional Repository. It has been accepted for inclusion in Electronic Theses and Dissertations by an authorized administrator of ThinkIR: The University of Louisville's Institutional Repository. This title appears here courtesy of the author, who has retained all other copyrights. For more information, please contact thinkir@louisville.edu. 
NOVEL ANALYTICAL TOOLS FOR STUDIES IN MOLECULAR ASSEMBLIES:

I) ELECTRO-ACTIVE SINGLE-MODE INTEGRATED OPTICAL WAVEGUIDES;

II) COUPLED PLASMON WAVEGUIDE RESONANCES

By

Xue Han

B.S., Dalian University of Technology, 2006

M.S., Dalian University of Technology, 2009

M.S., University of Louisville, 2014

\begin{abstract}
A Dissertation
Submitted to the Faculty of the

College of Arts and Science of the University of Louisville in Partial Fulfillment of the Requirements for the Degree of
\end{abstract}

\author{
Doctor of Philosophy \\ Department of Physics and Astronomy \\ University of Louisville \\ Louisville, Kentucky
}

December, 2014 
Copyright 2014 by Xue Han

All rights reserved 

NOVEL ANALYTICAL TOOLS FOR STUDIES IN MOLECULAR ASSEMBLIES:

I) ELECTRO-ACTIVE SINGLE-MODE INTEGRATED OPTICAL WAVEGUIDES;

II) COUPLED PLASMON WAVEGUIDE RESONANCES

By

Xue Han

B.S., Dalian University of Technology, 2006

M.S., Dalian University of Technology, 2009

M.S., University of Louisville, 2014

A Dissertation Approved on

November 20, 2014

by the following Dissertation Committee:

Dr. Sergio B. Mendes

Dr. Gamini Sumanasekera

Dr. Christopher L. Davis

Dr. Francis P. Zamborini

Dr. Robert S. Keynton

Dr. Kyung Aih Kang 


\section{ACKNOWLEDGEMENTS}

I would like to gratefully and sincerely thank Dr. Sergio B. Mendes. Without his patience, understanding and mentoring over the years, I would not be able to finish the research work and this dissertation. His philosophy has influenced me not only on academic research, but also on my attitude toward living a better life. Thank you!

By working in this multicultural research environment, my perspectives have been extended and broadened. I really appreciate the friendship that I obtained from the research group members, Scott Smith, Dr. Rodrigo S. Wiederkehr, Courtney Byard, Nathan Webster, Jennifer Burnett, Farzaneh Hoveyda, Jafar Ghitan, Ulyana Salgaeva, Adam Stewart, and Joseph Lynch.

I am also really grateful that Dr. Christopher L. Davis and Dr. Gamini Sumanasekera constantly gave me guidance, encouragement and help during my graduate period here.

At last I want to thank the finical support from the National Science Foundation and University of Louisville. 


\section{DEDICATION}

I want to specially thank Drs. Shi-yu Wu, Ming Yu and Shu-dun Liu. All the small talks made me feel I was home. I really appreciate that you all open the door for me when I needed suggestions.

I want to thank my dear friends. You became my family while I was away from home. Thank you all for listening and reaching out to help when I was having troubles, cheering me up when I felt blue and celebrating with me when I got even a small achievement. Thanks to Hongwei Gao, Li Zhou, Pin Zhang, Yan Ling, Yan Chen, Peng Xu, Qian Wang, Quiqi Liu, Baodong Hu and Xin Xu.

At last, I want to thank my parents. Their understanding and support provided me all the strength to go through the hard times. I don't have strong enough words to express my love and gratitude to them. 


\begin{abstract}
NOVEL ANALYTICAL TOOLS FOR STUDIES IN MOLECULAR ASSEMBLIES:

I) ELECTRO-ACTIVE SINGLE-MODE INTEGRATED OPTICAL WAVEGUIDES;

II) COUPLED PLASMON WAVEGUIDE RESONANCES
\end{abstract}

Xue Han

November 20, 2014

Two optical analytical tools were developed for studying molecular assemblies at solid/liquid interfaces. Electro-active single-mode integrated optical waveguide (EA-SM-IOW) technology was developed as a platform for spectroelectrochemical investigations on redox adsorbates at the sub-monolayer level. With an optimized ultra-thin indium tin oxide film combined with a singlemode integrated optical waveguide, for the first time, a more than 14,000 times higher sensitivity (compared to conventional potential-modulated transmittance) was achieved. From optical signals, this technique was able to reconstruct electrochemical information of redox adsorbates, including the formal potential and the electron transfer rate. A few major advantages were achieved with the developed EA-SM-IOW technology. I) As low as $10^{-15} \mathrm{~mol} / \mathrm{cm}^{2}$ electrochemically active surface coverage of redox species could be detected; II) A new analytical methodology was developed to combine optical impedance spectroscopy (OIS) with 
electrochemical impedance measurements to retrieve the electron transfer rate of redox process. This approach bypasses specific knowledge of every electrical element in the electrochemical flow cell; and III) The novel technique of OIS based on EA-SM-IOW platform was applied to examine the electron transfer processes of cytochrome c proteins under different environments that the surface densities were well below the limits of detection in conventional techniques. For molecular assemblies without a convenient absorbance band, a complementary analytical tool, coupled plasmon waveguide resonance (CPWR), was developed to detect surface binding events through changes in the real part of the refractive index and shown to be superior to the surface plasmon resonance (SPR), which has been considered as a gold standard technique. The advantages of CPWR included. I) When compared to SPR theoretically, the designed CPWR sensor was featured more than 30 times better resolution for the bulk solution changes and approximately 2 times superior for probing molecular surface adsorption; II) The smaller (and better) resolution of CPWR was demonstrated experimentally for the first time; and III) The CPWR was applied in an angle-multiplexed configuration to measure the fluorescence decay of a Ru-complex molecular assembly in the nano-second time scale. These two outstanding optical analytical tools provide unprecedented information and reach new detection limits that are expected to enable novel fundamental investigations and technological applications in molecular assemblies. 


\section{TABLE OF CONTENTS}

PAGE

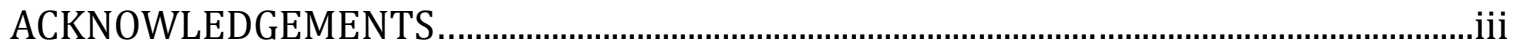

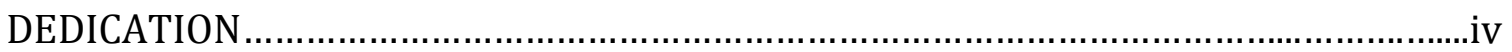

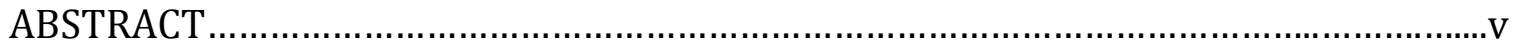

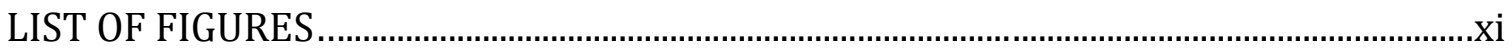

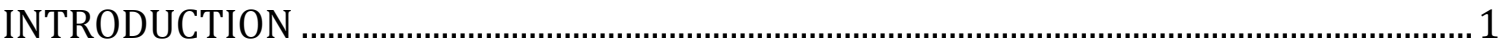

Challenges in Molecular Assembly Studies ........................................................ 1

Electro-Active, Single-Mode, Integrated Optical Waveguide Platform................. 2

Coupled Plasmon Waveguide Resonance Sensors ................................................... 7

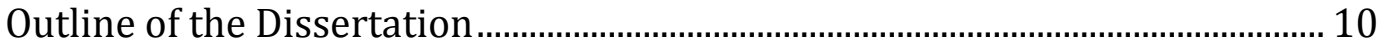

DESIGN, FABRICATION, AND OPTIMIZATION OF AN ELECTRO-ACTIVE, SINGLEMODE, INTEGRATED OPTICAL WAVEGUIDE ................................................................... 12

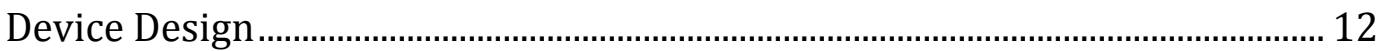

Deposition of Aluminum Oxide and Silicon Dioxide Layers ............................... 16

Indium Tin Oxide Deposition and Properties Characterization.......................... 17 
Device Performance Characterization

Conclusions 40

SPECTROSCOPIC FEATURES OF ULTRA-THIN ITO FILM UNDER POTENTIAL

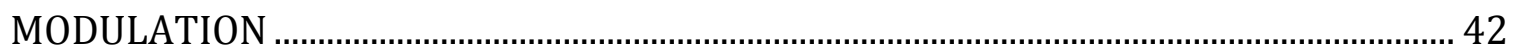

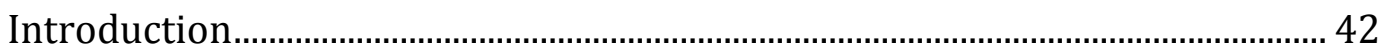

Spectroelectrochemical Flow Cell........................................................................... 43

Ultra-thin ITO under Potential Modulations........................................................... 45

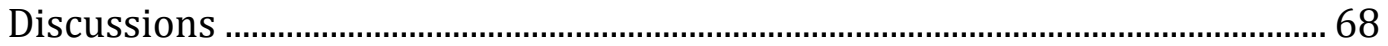

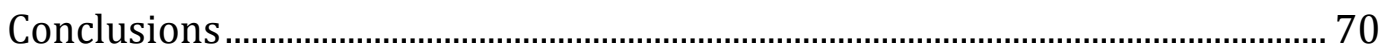

APPLICATIONS OF THE ELECTRO-ACTIVE, SINGLE-MODE, INTEGRATED OPTICAL WAVEGUIDE FOR STUDYING REDOX REACTIONS UNDER CYCLIC VOLTAMMETRY

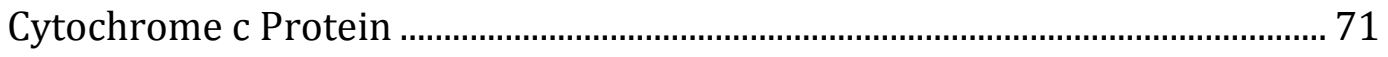

Experimental Processes and Results ......................................................................... 74

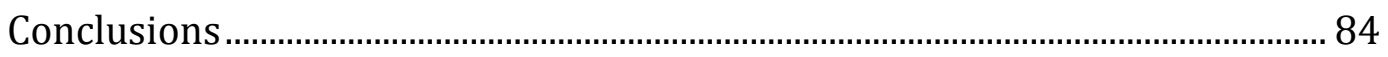

OPTICAL IMPEDANCE SPECTROSCOPY FOR KINETIC STUDIES OF REDOX

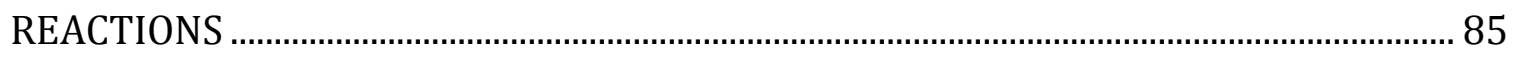

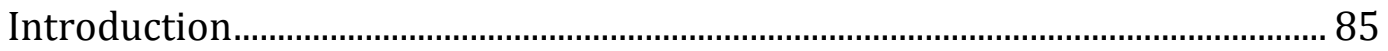

A Novel Analytical Methodology for Optical Impedance Spectroscopy …....... 92

Results from the New Optical Impedance Spectroscopy Analysis ..................100 
Conclusions

APPLICATIONS OF THE ELECTRO-ACTIVE, SINGLE-MODE, INTEGRATED OPTICAL WAVEGUIDE

Impact of Total Surface Coverage on Cytochrome c Adsorbates

Impact of Ionic Strength on Properties of Cytochrome c Adsorbates

Measurements of Electron Transfer Rate with Incident Light at Orthogonal

Polarization 140

Conclusions 144

COUPLED PLASMON WAVEGUIDE RESONANCE SENSOR: DESIGN AND FABRICATION 145

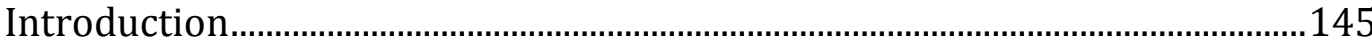

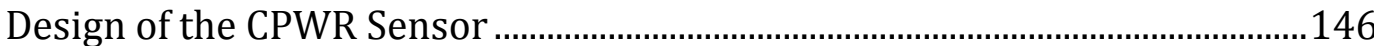

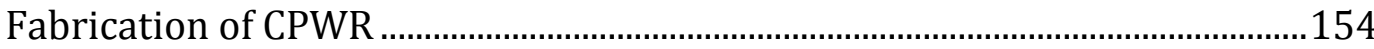

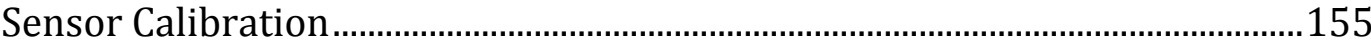

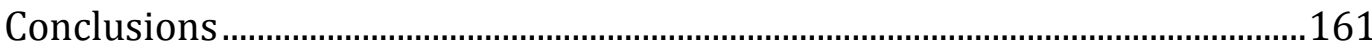

APPLICATION OF THE COUPLED PLASMON WAVEGUIDE RESONANCE....................163

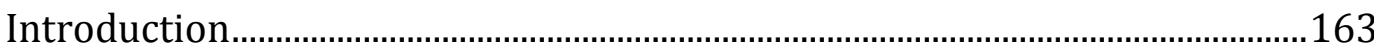

Angle-Multiplexed System ................................................................................... 163

Fluorescence Intensity Decay Experiment............................................................167 


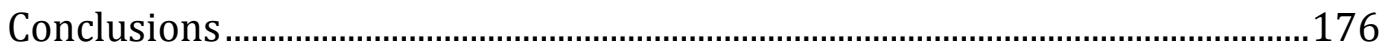

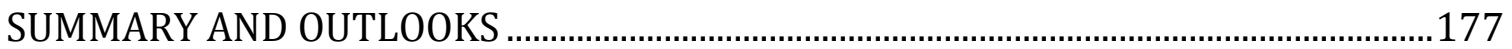

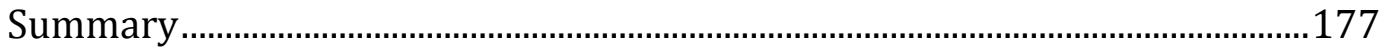

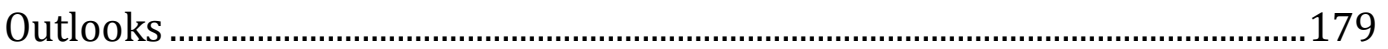

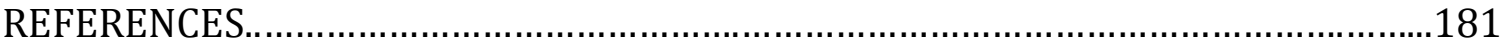

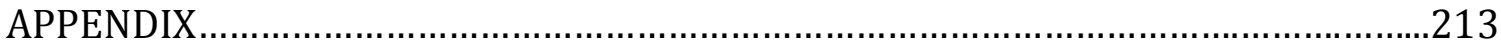

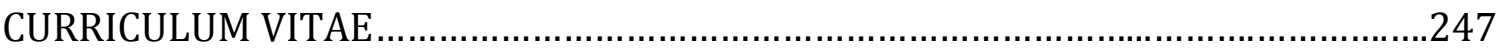




\section{LIST OF FIGURES}

Figure 1-1: Schematic figure for the potential-modulated attenuated total reflection.

Figure 2-1: The architecture of an electro-active single-mode integrated optical waveguide (not in scale). Two surface relief gratings with $323 \mathrm{~nm}$ pitch size are used as the light couplers. The structure of the single-mode multi-layer waveguide is formed by a $400 \mathrm{~nm}$ aluminum oxide, a $16 \mathrm{~nm}$ silicon dioxide, and a $13 \mathrm{~nm}$ transparent conductive indium tin oxide. Different probing wavelengths can be coupled into the system at its own coupling angle. With $2.54 \mathrm{~cm}$ probing length, the guided light is coupled out at the right side grating. A real out-coupled spectrum is shown in the inset. 15

Figure 2-2: Match of the experimental and the simulated transmittance curves to determine the thickness of the aluminum oxide and the silicon dioxide layers. The fitting parameters for the alumina film were $n_{A l_{2} O_{3}}=1.65$ and $t_{A l_{2} O_{3}}=409 \mathrm{~nm}$, and for silicon dioxide were $n_{\mathrm{Si}_{2}}=1.51$ and $t_{\mathrm{SiO}_{2}}=16 \mathrm{~nm}$.

Figure 2-3: Transmittance curves for ITO films as deposited under various $\mathrm{O}_{2}$ flow rate. The higher the $\mathrm{O}_{2}$ flow rate, the higher the transmittance is. At $0.7 \mathrm{sccm}$ and 0.8 sccm, the transmittance reaches saturation. 19 
Figure 2-4: Transmittance curves for ITO films as deposited with $0.6 \mathrm{sccm} \mathrm{O}_{2}$ flow rate and after the inert annealing process. After the annealing process, the film is more transparent in the visible range. 20

Figure 2-5: Extinction coefficient of the ITO films as sputtered and after the inert annealing process at various $\mathrm{O}_{2}$ flow rate during sputtering deposition. At $0.6 \mathrm{sccm}$, the lowest extinction coefficient is observed for the ITO film after the inert annealing process. 22

Figure 2-6: Resistivity of the ITO films as sputtered and after the inert annealing process with various $\mathrm{O}_{2}$ flow rate during sputtering deposition. A lower $\mathrm{O}_{2}$ flow rate during the sputtering resulted in a lower resistivity after the inert annealing process.

Figure 2-7: Real refractive index of thick ITO films before and after the inert annealing with $0.6 \mathrm{sccm} \mathrm{O}_{2}$ flow rate during the sputtering. Lower real refractive index after the inert annealing was observed. 24

Figure 2-8: Demonstration of the propagation loss and the coupling efficiency measurement set-up 25

Figure 2-9: Simulations of transmittance profile to determine the thickness of the ultra-thin ITO film. The fitting parameters for the alumina film were $n_{\mathrm{Al}_{2} \mathrm{O}_{3}}=$ 1.65 and $t_{\mathrm{Al}_{2} \mathrm{O}_{3}}=409 \mathrm{~nm}$, for silicon dioxide were $n_{\mathrm{SiO}_{2}}=1.51$ and $t_{\mathrm{SiO}_{2}}=16 \mathrm{~nm}$, and for the ITO film were $n_{\text {ITO }}=1.88$ and $t_{\text {ITO }}=18 \mathrm{~nm}$. Matches were observed between the simulation and the experimental results. 27 
Figure 2-10: Schematic representation of the experimental set-up for broadband light coupling. Two identical sets of optical elements are used to couple light into and out of the EA-SM-IOW. For the signal detection, an ICCD connected with a

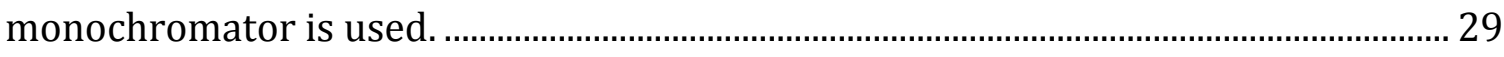

Figure 2-11: Broadband coupling for an EA-SM-IOW by using a tungsten-halogen lamp. A dark signal is plotted as the red curve. 30

Figure 2-12: Simulation results of the effective refractive index of the device in an aqueous environment 31

Figure 2-13: Simulation result of the field distribution of the device in the cross section. The interface of the alumina/glass slide locates at $z=0$, and red bar represents the layer of the $13 \mathrm{~nm}$ ITO film. 32

Figure 2-14: Absorbance measurement of a molecular thin film with (a) IOW probing structure and (b) transmittance probing structure. 34

Figure 2-15: Plot of Sensitivity against probing wavelength at TE polarization. For this simulation, the same parameters of the EA-SM-IOW used for the effective refractive index and electrical field distribution simulations were applied. 35

Figure 2-16: (a) Structure of the reactive blue dye; and (b) Structure of the dextran unit. 36

Figure 2-17: Absorbance curves of various bulk concentrations of blue dextran in DI water measured with the transmission probing structure. 37 
Figure 2-18: Absorbance curves for various bulk concentration of blue dextran in DI water measured with the EA-SM-IOW probing structure. 38

Figure 2-19: Absorbance plot against bulk concentration of blue dextran measured by EA-SM-IOW at $560 \mathrm{~nm}$ and $570 \mathrm{~nm}$. Linear fitting is used. The slope of the fitted results is used to calculate the sensitivity factor. 39

Figure 3-1: Cross section of the electrical contacts for an EA-SM-IOW in the spectroelectrochemical flow cell. Different materials used for the electrical connection package are demonstrated with different colors.

Figure 3-2: Experimental set-up with the stationary coupling set-up for the CV potential scan. A CV potential modulation with $0.02 \mathrm{~V} / \mathrm{s}$ scan speed is shown in the inset on top. 46

Figure 3-3: Out-coupled spectra at different potential points during the CV scan. ... 47

Figure 3-4: Rotation coupling set-up for the cyclic voltammetry potential scan with a laser beam as the light source. The coupling angle for each laser beam is achieved by rotating the stage. One trace of the out-coupled intensity against time collected by the ICCD camera is shown in the top inset. 48

Figure 3-5: (a) Normalized transmittance for different laser beams under the same $\mathrm{CV}$ potential scan. Potential modulation is plotted in the same graph against time as in black curve. Each curve is shifted by one unit. (b) Folded 5-cycles of normalized transmittance against the applied potential is plotted to show the high repeatability of the spectroscopic features. 50 
Figure 3-6: Electrical current curves under the same the CV potential scan when various laser beams are used as probing light sources. For the same device, the same CV scan results similar current curve. Anodic current is set as the positive current.

Figure 3-7: Out-coupled intensity against incident angle profiles by using (a) 514 $\mathrm{nm}$ and (b) $594 \mathrm{~nm}$ laser beams as probing light sources at different constant potential steps 54

Figure 3-8: Effective refractive index for an EA-SM-IOW electrode in neutral buffer solution at various constant potential steps. 55

Figure 3-9: Normalized transmittance comparison between the CV scan and the constant potential steps for (a) $514 \mathrm{~nm}$ and (b) $594 \mathrm{~nm}$. 57

Figure 3-10: Experiment set-up for the AC potential modulation measurement. A super continuum fiber laser source is used as the light source and an acousto-optical filter is used to select probing wavelength. The out-coupled light intensity is collected by a PMT and amplified by a pre-current amplifier. The potential modulation signal and the optical response from the spectroelectrochemical flow cell are recorded simultaneously by an oscilloscope. 58

Figure 3-11: (a) AC potential modulation signal at - $0.8 \mathrm{~V}$ DC bias and (b) the optical out-coupled intensity signal with $550 \mathrm{~nm}$ probing wavelength. 60

Figure 3-12: Optical DC component intensity for $550 \mathrm{~nm}$ and $580 \mathrm{~nm}$. 61 
Figure 3-13: The comparison of normalized transmittance between the optical DC components from the $\mathrm{AC}$ impedance potential modulation and the $\mathrm{CV}$ potential scan used a tungsten-halogen white light source. A match is observed for each probing wavelength (a) $550 \mathrm{~nm}$ and (b) $580 \mathrm{~nm}$. 62

Figure 3-14: The optical AC amplitude against the potential oscillation frequency for (a) $550 \mathrm{~nm}$ and (b) $580 \mathrm{~nm}$ at different potential DC biases. 64

Figure 3-15: Complex planes from different potential DC biases for (a) $550 \mathrm{~nm}$ and (b) $580 \mathrm{~nm}$. 66

Figure 3-16: Electrical impedance results of AC potential modulation measured by the potentiostat. (a) The total impedance amplitude and (b) the phase delay against the oscillation frequency. 67

Figure 3-17: Picture of thicker ITO films under a CV potential modulation in $4.5 \mathrm{pH}$ acid solution for sputtered sample with $0.55 \mathrm{sccm}$ and $0.6 \mathrm{sccm}$. 69

Figure 4-1: Calibrated molar absorptivity for the oxidized and the reduced states of cytochrome c proteins in buffer solution. 73

Figure 4-2: (a) CV potential scan of 5 cycles between $-0.4 \mathrm{~V}$ and $+0.4 \mathrm{~V}$ with 0.02 $\mathrm{V} / \mathrm{s}$ speed, (b) the optical reference signal, and (c) the optical signal from cytochrome c proteins contained system under the same CV potential scan from 550 nm probing wavelength. 76

Figure 4-3: Absorbance curves from three wavelengths $550 \mathrm{~nm}, 557 \mathrm{~nm}$ and $580 \mathrm{~nm}$ for cytochrome c proteins under a CV potential scan. 78 
Figure 4-4: The 5-cycle of faradaic current density reconstructed by the optical absorbance signal from $550 \mathrm{~nm}$. Both potential scan directions are included. ..........8 80

Figure 4-5: Gaussian fitting for one segment of the reconstructed faradaic current dentistry against the potential curve. 81

Figure 4-6: Electrical current density calculated by using the potentiostat measured electrical current signal and an effective area of the EA-SM-IOW surface. No difference can be observed between the reference and the protein signals. 83

Figure 5-1: An equivalent electrical circuit for an electrochemical flow cell with a molecular thin film immobilized on the working electrode surface. The electrolyte solution has a resistance, $R_{s}$. The equivalent electrical circuit for the redox reaction of the surface-adsorbates is represented by a charge transfer resistance, $R_{c t}$, in series with a pseudo capacitance, $C_{a}$. And this circuit is in parallel with the double layer capacitance, $C_{d}$ 86

Figure 5-2: Electrochemical impedance measurements and simulation results for flow cell with and without protein presence. In A, both the real and imaginary components of the current are plotted against potential. In B, experimental results of the real and imaginary components of impedance spectroscopies for the electrochemical flow cell without and with redox species are plotted against modulation frequency. The simulation results by using the equivalent electrical circuits are plotted to match the experiment results. 91

Figure 5-3: Generalized equivalent electrical circuit for the spectroelectrochemical flow cell. Impedance of the electrochemical flow cell is generalized with $Z_{1}$ and $Z_{2}$, 
which includes the resistance of the electrolyte solution and other possible

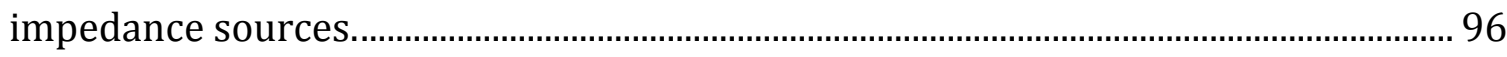

Figure 5-4: Optical response at $550 \mathrm{~nm}$ from cytochrome c protein contained system under AC impedance potential modulations for various potential DC biases.

Figure 5-5: DC component of the optical out-coupled intensity at each DC potential bias with average over the frequencies for optical baseline and cytochrome c protein contained optical signals.

Figure 5-6: (a) DC component of absorbance against potential DC bias. (b) Reconstructed relative faradaic current density profile with a Gaussian fit. .104

Figure 5-7: Complex planes comparison between the optical reference signal and the proteins signal. The blue diamonds are for the reference signal. The red squares are for cytochrome c proteins signal. All 6 graphs are plotted with the same scale to show differences. The oscillating frequency increases in the clockwise direction, as shown in the plot of the $-0.2 \mathrm{~V}$ potential DC bias. The AC component of the absorbance is represented by a black arrow the drawn from optical baseline to the proteins signal with the same frequency. The real component and imaginary component of the AC absorbance are represented by a purple and a green arrow. .106

Figure 5-8: With the left side y-axis, the active surface coverage of cytochrome c proteins under $15 \mathrm{mV} \mathrm{AC}$ amplitude of impedance potential modulations for each potential DC bias is plotted against angular modulation frequency. With the right side $y$-axis, the AC absorbance results are plotted. .108 
Figure 5-9: Reconstructed faradaic current density for each potential DC bias is plotted against the angular frequency. 109

Figure 5-10: Reconstructed faradaic current density against the potential by selecting faradaic current value at $50 \mathrm{rad} / \mathrm{s}$ angular frequency as the blue dots. Gaussian fit is used as in the red solid line.

Figure 5-11: Electron transfer rate calculation for potential DC bias - $0.08 \mathrm{~V}$ (a) and $0.12 \mathrm{~V}$ (b). Linear fit is used, and the slope represents the electron transfer rate..112 Figure 5-12: Complex planes of the experimental and simulation results of faradaic admittance at $-0.8 \mathrm{~V}$ and $-0.12 \mathrm{~V}$ potential DC biases. Match is observed. 113

Figure 5-13: Comparison of electrical impedance results between the electrochemical flow cell without (blue diamond) and with cytochrome c (red square) presence. (a) Real component of the total electrical impedance. (b) Imaginary component of the total electrical impedance. (c) Phase delay. Data from $0.08 \mathrm{~V}$ potential DC bias is selected.

Figure 5-14: The comparison between the reference system and the cytochrome c contained system in optical complex planes for AC absorbance examination. Blue diamond represents the reference optical single and the red square represents the protein contained system for (a) $550 \mathrm{~nm}$ and (b) $570 \mathrm{~nm}$. 118

Figure 5-15: Linear fitting for electron transfer rate calculation for (a) $550 \mathrm{~nm}$ and (b) $570 \mathrm{~nm}$ using optical impedance spectroscopy results. 120

Figure 6-1: Experimental set-up for optical impedance measurement. 123 
Figure 6-2: Total surface coverage during the CV potential scans monitored by an isosbestic wavelength, $557 \mathrm{~nm}$. The higher the bulk concentration of the cytochrome c protein, the higher total surface coverage is resulted. For one bulk concentration of cytochrome c proteins, the total surface coverage is a constant during the potential modulation. 125

Figure 6-3: Experimental results of the surface coverage under different bulk concentrations with a Langmuir fitting. 126

Figure 6-4: (a) Absorbance data from $550 \mathrm{~nm}$ wavelength during CV scan for each total surface coverage, (b) reconstructed faradaic current density profiles by using absorbance signals from $550 \mathrm{~nm}$, and (c) average of faradaic current density peaks against total surface coverage under the same CV scan.

Figure 6-5: Reactive percentage of the surface immobilized cytochrome c against the total surface coverage. 130

Figure 6-6: (a) Formal potential and (b) full width at half maximum of the faradaic current density profiles of cytochrome c proteins under different total surface coverage. The average of 6 segments of the CV scan is used with the standard devotion as the error bars. 131

Figure 6-7: Reconstructed faradaic current density profiles from (a) DC and (b) AC components of absorbance data from OIS measurements. The dotted curves are from experimental results and the solid curves are Gaussian fits. 132

Figure 6-8: Formal potential for cytochrome $\mathrm{c}$ proteins redox reactions against total surface coverage. The DC (blue diamond) and AC components (red square) of the 
optical impedance data at $4 \mathrm{~Hz}$ are used to reconstruct the faradaic current density profile and examined with Gaussian fitting.

Figure 6-9: Full width at half maximum of the reconstructed faradaic current profiles from (a) DC and (b) AC components of absorbance data by selecting optical impedance data at $4 \mathrm{~Hz}$ and Gaussian fitting....... 135

Figure 6-10: Electron transfer rates for different total surface coverage of cytochrome c proteins redox reactions.

Figure 6-11: Electron transfer rate of cytochrome c protein in different ionic strength solutions. The $\mathrm{x}$-axis is the common logarithm of the ratio the concentration of salt over the bulk concentration of cytochrome $c$. the $x$ value is set as - 1 for the case that pure phosphate buffer is used.

Figure 6-12: Electron transfer rate calculation for both (a) TM and (b) TE polarized incident light. The electron transfer rate is represented by the slope of the linear fit.

Figure 7-1: Demonstration of the parameters in the definition of resolution for both techniques. 147

Figure 7-2: Calculated reflectivity curves for CPWR and SPR working in water solution. CPWR results a much narrower curve than the SPR. The best configuration for each sensor is used. 148

Figure 7-3: Calculation results of reflectivity against incident angle for (a) SPR and (b) CPWR under a 0.001 refractive index change in bulk solution. 150 
Figure 7-4: Calculation results of reflectivity curves for $0 \mathrm{~nm}, 1 \mathrm{~nm}, 2 \mathrm{~nm}$ and $3 \mathrm{~nm}$ molecular thin film adsorption on the surface of (a) SPR and (b) CPWR devices...152 Figure 7-5: Picture of the final CPWR sensor. Half of the area was coated with silver as the CPWR sensing area. The other half area was used to collect the total reflection intensity beyond the critical incident angle. 155

Figure 7-6: Traditional angular interrogation method for the CPWR device performance test. 156

Figure 7-7: Thickness examination of silver and silicon dioxide layers. TM polarized incident light is used to excite SP wave. 157

Figure 7-8: The thickness examination for the layer of silver and silicon dioxide. The blue dots are experimental results from the angular interrogation measurement. The pink solid line is simulation result with a $40.6 \mathrm{~nm}$ silver layer and a $6.9 \mathrm{~nm}$ silicon dioxide layer in air. 158

Figure 7-9: Thickness examination of aluminum oxide layer. TE polarized incident light is used to excite waveguide resonance. 159

Figure 7-10: Reflectivity against incident angular profile of the CPWR device in air. The blue dots are experimental results from the angular interrogation measurement. And the yellow solid line is the simulation result with a $40.6 \mathrm{~nm}$ silver layer, a 6.9 $\mathrm{nm}$ silicon dioxide layer and a $151.6 \mathrm{~nm}$ aluminum oxide layer in air. .160 Figure 7-11: Reflectivity against incident angle of CPWR device working in water solution. Blue dots are experimental results from the angular interrogation 
measurement. And the red solid line is the simulation result with a $40.6 \mathrm{~nm}$ silver layer, a $6.9 \mathrm{~nm}$ silicon dioxide layer and a $151.6 \mathrm{~nm}$ aluminum oxide layer in water.

Figure 8-1: Set-up of the angle-multiplexed system.

Figure 8-2: Pictures of the ICCD images for the angular resonance when a CPWR sensor working in an aqueous solution. 165

Figure 8-3: Reflected intensity against the pixel number profiles for the CPWR sensing area and the reference area. 166

Figure 8-4: Reflectivity curves measured by the angle-multiplexed system compared to the experimental result from the conventional angular interrogation and simulation result for the same CPWR device in an aqueous environment

Figure 8-5: Chemical expression of Ru-complex.

Figure 8-6: Top view of the flow cell mounted on a rotation stage. The blue arrow represents the excitation pulsed laser beam. This beam is aligned with the He-Ne laser beam at the same spot on the CPWR sensor. 170

Figure 8-7: Resonance angle shift for the Ru-complex molecule adsorption monitoring. 172

Figure 8-8: Fluorescence intensity profiles for TE polarized incident light at different time delay. 174

Figure 8-9: Exponential fitting for the time constant of Ru-complex fluorescence decay by using the height of the dip from each time delay. 175 
Figure 8-10: Fluorescence intensity profiles for TM polarization at different time

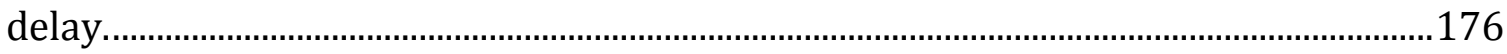




\section{CHAPTER 1}

\section{INTRODUCTION}

\section{Challenges in Molecular Assembly Studies}

For biological processes at the molecular level, the lipid membrane layer/cell fluids interface can be modeled by a solid/liquid interface. The targeted molecules are immobilized on the surface of an analytical tool, and their behaviors can be studied with or without stimulations for fundamental research and technological applications. Based on this model, the structural modification of a protein during folding and unfolding processes, the electron transfer rates and pathways for redox proteins, and the electrical potential difference between the membrane layers for ions transportation can be studied [1-7].

For molecular assembly studies, the weak signal is a major challenge. The concentration of molecules of interest can be extremely low in bio-systems, and many only have a subtle structural change during the biological processes. In diagnosing the early stage of diseases invariably the concentrations of the targeted molecules are extremely low, and these features are critical. Recognition elements, such as antibodies, can be used to increase the selectivity and affinity to surface binding events. Even with these elements, enough sensitivity for detection of bio- 
recognition events is still a challenge in bio-analytical chemistry [8]. For biomolecular structural-function studies, surface enhanced techniques have been applied to increase the sensitivity [9-14]. Surface enhanced infrared absorbance spectroscopy (SEIRAS) has the ability to detect approximately $10^{-12} \mathrm{M}$ concentration of targeted molecules [1]. Commercial tools, based on surface plasmon resonance (SPR) (e.g. BIAcore 4000) and a diffraction grating (SRU biosystems, BIND), have the sensitivity at $0.01 \mathrm{ng} / \mathrm{mm}^{2}[15,16]$. For a typical protein, this sensitivity is equivalent to approximately $10^{-13} \mathrm{~mol} / \mathrm{cm}^{2}$. For molecular assemblies at sub-monolayer concentrations, analytical tools with higher sensitivity are needed to differentiate the minute signal over the background noise.

To overcome this challenge, a single-mode optical waveguide is a suitable base for the architecture of optical analytical tools. A single-mode optical waveguide has a tight confinement of the guided wave. Due to the long path length of the interaction between the evanescent field and the surface adsorbed species, a very high sensitivity can be achieved. Here, two optical analytical tools featured with single-mode optical waveguide architecture are developed. Their capability in detecting and characterizing molecular assemblies at sub-monolayer concentration levels are demonstrated.

\section{Electro-Active, Single-Mode, Integrated Optical Waveguide Platform}

Electrochemical studies of electron transfer processes for redox proteins in bio-systems are crucial to understand their biological roles [17-27]. For example, 
cytochrome c protein transfers electrons between the cytochrome c reductase and oxidase complexes in the respiratory system.

For the surface immobilized redox species, when a potential modulation is applied to an electrode, an electrically driven chemical reaction (faradaic process) can occur with electrons exchanges between the electrode and the adsorbed molecules. This electron transfer process generates a specific electrical current, referred to as the faradaic current. The traditional electrochemical techniques (amperometric, voltammetric, and impedimetric) directly analyze the electrical current signal, and spectroelectrochemical techniques analyze the transduced optical signal.

\section{Traditional electrochemical techniques}

In traditional electrochemistry, the detection sensitivity is usually limited by a strong background current signal from the flow of electrolyte ions under potential modulations. For a sub-monolayer of redox proteins, the faradaic current signal is too small and is buried in the background, as the cyclic voltammetry (CV) cannot resolve the faradaic current peaks at such low concentrations for redox species undergoing electrochemical reactions.

Electrochemical impedance spectroscopy (EIS) is a widely used technique because of its sensitivity [28-32]. A series of alternating current (AC) potential modulations are used to drive the electrochemical flow cell, and only the current signal at the same frequency as the driving potential modulation is recorded. By this way, noise is substantially reduced and the detection sensitivity is improved. Other 
modulation techniques such as the square wave voltammetry [33-35], single potential step [36] and multi-pulse voltammetry [37, 38] are also widely used to improve the sensitivity. Besides different potential modulation techniques, nanosized electrodes have also been developed to improve the sensitivity [39-45].

Although various potential modulation schemes and engineered electrodes have been developed, the sensitivity of directly using the electrical current signal to monitor electrochemical reactions in a monolayer or a sub-monolayer of redox species is still problematic in many studies.

\section{Spectroelectrochemistry}

Optical probing techniques have been coordinated with electrochemistry to monitor electron transfer processes to increase the detection sensitivity. The rationale for spectroelectrochemistry is as follows. For a redox molecule with two different absorbance spectra for the reduced and oxidized states, the wavelength of the incident light can be tuned to probe the faradaic process. Since the ions of the electrolyte solution typically do not absorb the incident light, the optical signal is not influenced by this electrical background. By avoiding this strong background signal, the detection sensitivity has been increased enormously, and the electrochemical reaction of a single molecule is detectable $[46,47]$.

Besides a higher sensitivity, there are also other benefits provided by spectroelectrochemical techniques. I) Spectroscopic measurements can be applied. A wavelength with a large difference in molar absorptivity between the two redox states can be selected to monitor the faradaic process. And simultaneously, the 
isosbestic wavelength (the reduced and oxidized states of the molecule have the same molar absorptivity) can be selected to monitor the total surface coverage during electrochemical modulations. II) Different polarizations of the incident light can be used to investigate possible correlations of the molecular order (i.e. orientation) and the electron transfer behaviors. III) A superior selectivity can be achieved. For a mixture of molecules, besides the potential window that can be selected to trigger one species to have redox reaction, the probing wavelength can be tuned to detect one type of species. Spectroelectrochemical techniques also have a huge potential to study multiple electron transfer mechanisms. The conventional electrochemistry is limited to the overall electrical process and is not capable of discerning details of the different electron transfer channels.

The potential-modulated transmittance and electro-reflectance methods are straightforward to be implemented [48-54]. However, because these analytical techniques involve only one time interaction (single pass transmission or single bounce reflection) between the probing light beam and the redox species, their sensitivity is insufficient for detection in most cases with a surface density at the monolayer or sub-monolayer level.

Potential-modulated attenuated total reflection (PM-ATR), which is considered as a multi-mode waveguide geometry, has approximately 100 times higher sensitivity [55-61] than potential-modulated transmittance measurements. In Figure 1-1, the PM-ATR is illustrated [60]. 


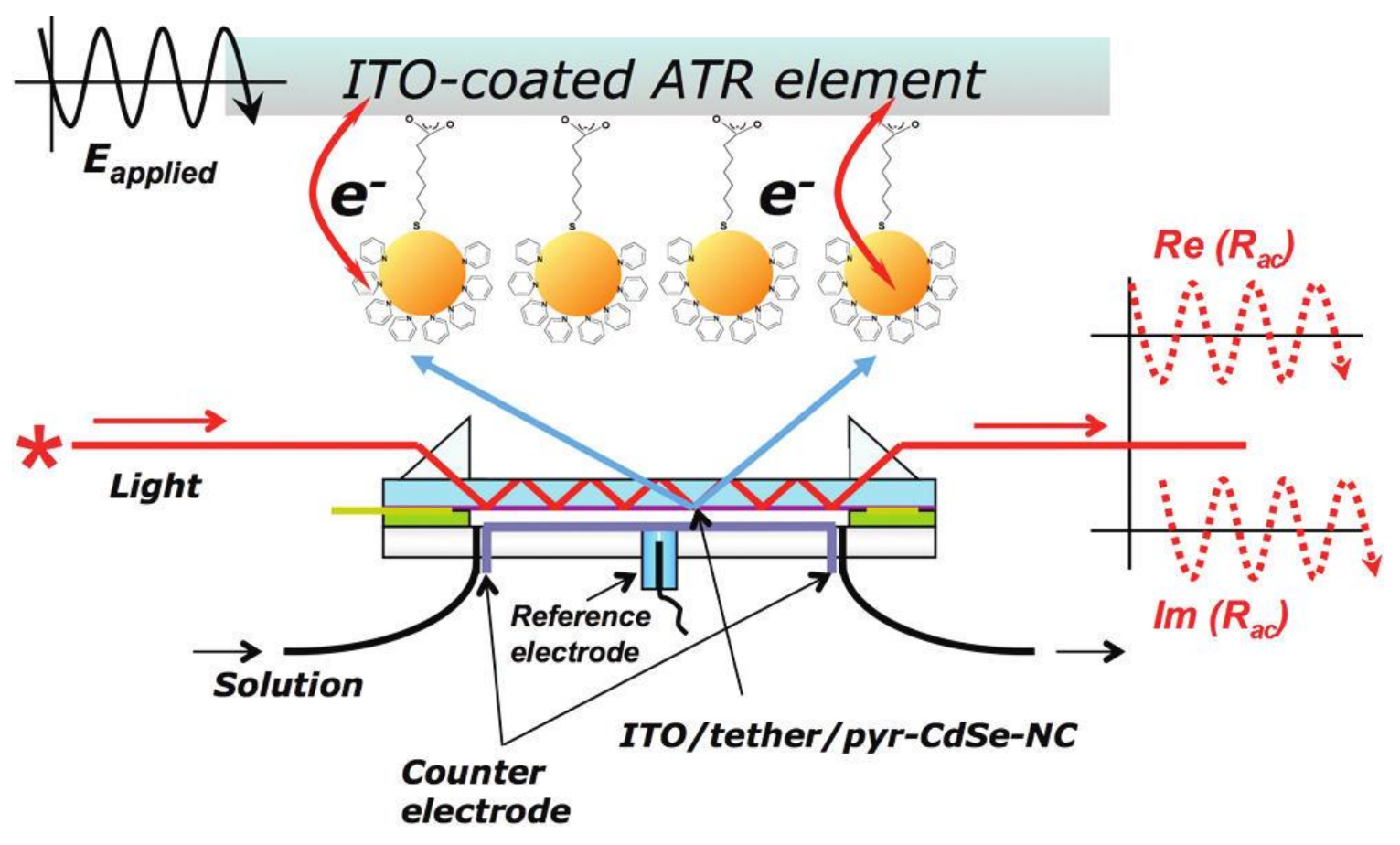

Figure 1-1: Schematic figure for the potential-modulated attenuated total reflection. Reprinted with permission from "Potential-Modulated Attenuated Total Reflectance Characterization of Charge Injection Processes in Monolayer-Tethered CdSe Nanocrystals. Journal of Physical Chemistry Letters, 2010. " Copyright (2010) American Chemical Society.

Other optical analytical techniques such as fluorescence [62] and Raman spectroscopy [41, 63-67] have been combined with electrochemical potential modulations. The limiting factor for the sensitivity is the interaction distance between the probing light beam and the redox species (usually the thickness of the molecular assembly). 
Compared to the PM-ATR, a more sensitive optical analytical tool is desired for detection and characterization of redox reactions at sub-monolayer levels. An electro-active single-mode integrated optical waveguide (EA-SM-IOW), which has strong confinement of the guided wave and ten thousand times higher response compared to the potential-modulated transmittance, is a proper candidate.

\section{Coupled Plasmon Waveguide Resonance Sensors}

When a proper absorbance band is not applicable, SPR is a sensitive analytical technique to detect changes in real refractive index introduced by molecular assemblies processes. SPR sensors are applied widely in new medicine designs and catalysis process studies. It is also combined with other microscopic analytical techniques to enhance the detection signal [68].

Traditional surface plasmon resonance sensors

Surface plasmon (SP) is a free electric charge oscillation that can propagate along the interface of a dielectric medium and a metallic medium with a negative dielectric constant (e.g., $\mathrm{Au}, \mathrm{Ag}, \mathrm{Cu}$ ). This surface wave features an electromagnetic field profile that is highly confined to the dielectric/metal interface. SP wave can be excited, when the propagation constant (also known as the $k$ vector) of the incident light (with a transverse magnetic polarization) matches to the one of the SP wave. At this resonant condition, the power of the incident light is transferred to excite the SP wave, and the reflected light is at minimum. This condition (i.e. the propagation constant) is extremely sharp (in angular or wavelength scanning). For a small 
detuning from the resonant condition, the reflectance rapidly increases from its minimum value. For this reason, a small change in the dielectric constant near the metal/dielectric interface will drastically shift the resonant condition. By measuring the optical parameters associated with the change in the resonant condition, SPR technique is used to detect molecular assemblies.

To measure the resonance condition shift, the angular interrogation is popular because of its simplicity. Optical properties of the dielectric and the metallic media for only one incident wavelength are needed. A monochromatic incident light from a laser is usually used to excite a SP wave and the intensity of the reflected light is measured against the incident angle. As mentioned above, the angle of the strongest coupling results in the minimum reflected intensity, since the energy at this coupling angle is transferred into SP wave [69-71]. The dip shifting represents a coupling condition change.

The wavelength [72-84], intensity [85] and phase interrogation methods [86, 87] can also be used for SPR sensors according to different circumstance of applications.

Even though the conventional SPR biosensors are more sensitive than most other label-free technologies (i.e. ellipsometry, quartz crystal microbalance, etc.), they are still unable to directly detect molecules with small molecular weight (few hundreds of Daltons) or at low surface concentration. Modifications have been made on the traditional SPR sensors to improve the sensitivity and versatility [88-90], 
such as the long-range SPR (LRSPR) [91-94], coupled plasmon waveguide resonance (CPWR) [95-99], and nano-structure enhanced SPR [100-107].

Motivation of coupled plasmon waveguide resonance

An interesting alternative of the conventional SPR device is made by overlaying a transparent dielectric film atop of a noble (typically $\mathrm{Au}$ or $\mathrm{Ag}$ ) metal layer. The approach is known in the literatures as the waveguide resonance, optical waveguide spectroscopy, plasmon-waveguide resonance, or coupled plasmonwaveguide resonance (CPWR). In terms of the operation, the CPWR device works similarly to the SPR sensor. For example, in the Kretschmann geometry, the incident light from a prism is coupled to the waveguide mode at the specific angle of resonance. Although the majority of the electromagnetic field of the guided wave is inside the transparent dielectric material, a small fraction still penetrates inside the metal film. The absorption of the metal film creates a dip in the reflectance profile with a much narrower angular width (compared to SPR) due to a longer propagation length. Besides a stronger factor of the quality for the reflectance resonance and a higher sensitivity, other features of this configuration can be beneficial. I) While the SPR only can be excited by a transverse magnetic polarized light, both transverse electric and magnetic modes can be supported by a CPWR device. Such dual-polarization capability offers possibilities of birefringence studies $[108,109]$. II) The choice of the waveguide material is not restricted, as long as the optical characteristics are favorable. The waveguide layer can be a configuration of a few different layers to optimize different applications. III) The waveguide layer also 
can serve as a protection for the metallic thin film against mechanical or chemical damages.

Although several important applications have been reported in the literatures $[69,83,90,110-112]$, the experimental demonstration of the ideal CPWR performance has been hampered $[76,96,97]$. The major difficulty is to create a high quality optical waveguide (i.e., a dielectric film has extremely high transparency and a very smooth surface) of precise thickness over a soft noble metal film. In this work, the atomic layer deposition (ALD) technique is used to coat a high quality optical waveguide on top of a thin metal layer for a novel CPWR device.

\section{Outline of the Dissertation}

In Chapter 2, the design, fabrication and optimization of the EA-SM-IOW are described. For the transparent conductive layer, an optimized ultra-thin indium tin oxide (ITO) film is coated on a single-mode integrated optical waveguide. An extremely low extinction coefficient (to minimize propagation loss) and a low resistivity (to support potential modulations) of an EA-SM-IOW device are achieved. Both the theoretical simulation and experimental results show that the detection sensitivity is 14,000 times higher than the potential-modulated transmittance technique. In Chapter 3, the optical and electrical features of the ultra-thin ITO film under potential modulations are described. Various potential modulation techniques are applied, and the spectroscopic features are consistent. To study a redox reaction of one molecular assembly, these optical baselines (features) should be factored out. In Chapter 4, a sub-monolayer of cytochrome c proteins is examined 
by a CV potential scan based on the EA-SM-IOW platform. The superiority of the optical probing strategy over the conventional electrochemical $\mathrm{CV}$ technique is demonstrated. The total surface coverage and active surface coverage are calculated based on the spectroscopic measurement during the CV potential scan. In Chapter 5 , to study the charge transfer kinetics of a surface immobilized redox assembly at sub-monolayer level, an optical impedance spectroscopy (OIS) is applied. A new analytical methodology is developed and demonstrated. The optical baseline is factored out, and as low as $10^{-15} \mathrm{~mol} / \mathrm{cm}^{2}$ of the active cytochrome c protein is detected. A strategy is developed to calculate the electron transfer rate in a simple and accurate manner. In Chapter 6, the EA-SM-IOW platform combined with the new OIS methodology is applied to study the redox reaction kinetics of cytochrome c proteins at sub-monolayer level under various environments. In Chapter 7, the design, fabrication, and characterization of the device CPWR are demonstrated. Theoretical comparisons are carried out between the conventional SPR sensor and the CPWR device. Changes in the bulk refractive index and the adsorption of a molecular film are considered, and the CPWR sensor shows smaller (and better) resolutions in both cases. In Chapter 8, a set-up of an angle-multiplexed measurement is introduced to combine with the CPWR sensor to detect the fluorescence decay in real-time (nano-second time scale). In chapter 9, concluding considerations are presented and an outlook is offered for possible applications of these novel optical tools. 


\title{
CHAPTER 2
}

\section{DESIGN, FABRICATION, AND OPTIMIZATION OF AN ELECTRO-ACTIVE, SINGLE-MODE, INTEGRATED OPTICAL WAVEGUIDE}

\begin{abstract}
An electro-active single-mode integrated optical waveguide (EA-SM-IOW) can provide a strong light confinement and a long interaction distance (approximately $\mathrm{cm}$ ) between the guided light and the molecular film. In this chapter, the design, fabrication and optimization of an EA-SM-IOW device are described. Both the theoretical simulation and experimental results demonstrate that this optical tool has a higher sensitivity (14000 times) when compared to the conventional potential-modulated transmittance technique.
\end{abstract}

\section{Device Design}

Multi-layer design for the single-mode optical waveguide

To allow proper operation of electrochemical potential modulations and optical measurements, a single-mode optical waveguide coated with a transparent conductive electrode needs to meet three major requirements. I) The attenuation of the guided light from the optical waveguide layer should be minimized to provide a strong probing signal. For this purpose, an atomic layer deposition (ALD) technique 
was used to coat an aluminum oxide layer that provided an extremely low optical loss for the guided light propagation [113]. II) To provide a high response for absorbance measurements of the surface-adsorbed redox species under potential modulations, the guided light should have a sufficiently large electric field at the probing surface. By adjusting the thickness of the single-mode waveguide (mainly the alumina layer), a tradeoff between the optical loss (attenuation) from the transparent conductive indium tin oxide (ITO) layer and the electric field at the electrode surface was adjusted. The multi-layer structure of the EA-SM-IOW is shown in Figure 2-1. The silicon dioxide layer was designed to protect the aluminum oxide layer. Possible ion migration could be generated by the ITO deposition and annealing processes. A Mathematica program based on a transfer-matrix technique was used to calculate TE mode electrical field profile for this multi-layer structure (Appendix 1) in the visible spectral region. Different thicknesses of the waveguide layer were tested. To adjust the tradeoff discussed above, a layer of $400 \mathrm{~nm}$ alumina was selected for the final design. III) The transparent conductive layer should be transparent enough to minimize the optical attenuation of the guided light, and sufficiently conductive to minimize the potential drop for the electrochemical applications [114]. Obtaining a good optical transparency and electrical conductivity simultaneously for the ITO film was the crucial challenge for the successful implementation of this optical analytical tool. The optimization for the ITO layer is described in Section 2.4 . 


\section{Diffraction grating, the light coupler}

Two surface relief gratings were designed as the light couplers. The distance between them was chosen to be $2.54 \mathrm{~cm}$ (or $3.4 \mathrm{~cm}$ for certain devices) to provide a long light/material interaction distance to assure a high sensitivity. Each probing wavelength requires a specific incident angle to be coupled into the IOW by a diffraction grating. This angle (known as the coupling angle) can be calculated for each wavelength with Equation 2-1, where $N_{\text {eff }}$ is the effective refractive index of the multi-layer structure, $n_{\text {in }}$ is the refractive index of the incident medium, $\theta_{\text {couple }}$ is the coupling angle, $\lambda$ is the wavelength of the incident light, $m$ is the order of the diffraction from the grating, and $\Lambda$ is the pitch size of the grating. For the EA-SMIOW, the gratings were designed and fabricated at $323 \mathrm{~nm}$. The fabrication of the surface relief grating is described in Appendix 2.

$$
N_{\text {eff }}=n_{\text {in }} \sin \theta_{\text {couple }}+m \frac{\lambda}{\Lambda}
$$

At $550 \mathrm{~nm}$ incident light, $N_{\text {eff }}$ is 1.59702 (Appendix 1) with the calculation parameters for the incident medium $\left(n_{\text {in,air }}=1\right.$ and $\left.t_{\text {in, air }}=\infty\right)$, the aluminum oxide layer $\left(n_{\mathrm{Al}_{2} \mathrm{O}_{3}}=1.64\right.$ and $\left.t_{\mathrm{Al}_{2} \mathrm{O}_{3}}=400 \mathrm{~nm}\right)$ [113], the silicon dioxide layer $\left(n_{\text {SiO }_{2}}=1.51\right.$ and $\left.t_{\text {SiO }_{2}}=16 \mathrm{~nm}\right)$, the ITO layer $\left(n_{\text {ITO }}=1.9\right.$ and $\left.t_{\text {ITO }}=13 \mathrm{~nm}\right)$, and the cladding medium $\left(n_{c, \text { water }}=1.33\right.$ and $\left.t_{c}=\infty\right)$. With Equation 2-1, the coupling angle for $550 \mathrm{~nm}$ is approximately - 6 degree for the diffraction order of 1. In Figure $2-1$, a broadband incident light with a range of incident angles, which is schematically represented by the yellow color, is coupled into the waveguide from 
the grating coupler on the left side. Each wavelength is coupled at its own coupling angle with the best efficiency. After propagation along the device, the guided light is out-coupled from the grating coupler on the right side and a color separation is resulted.

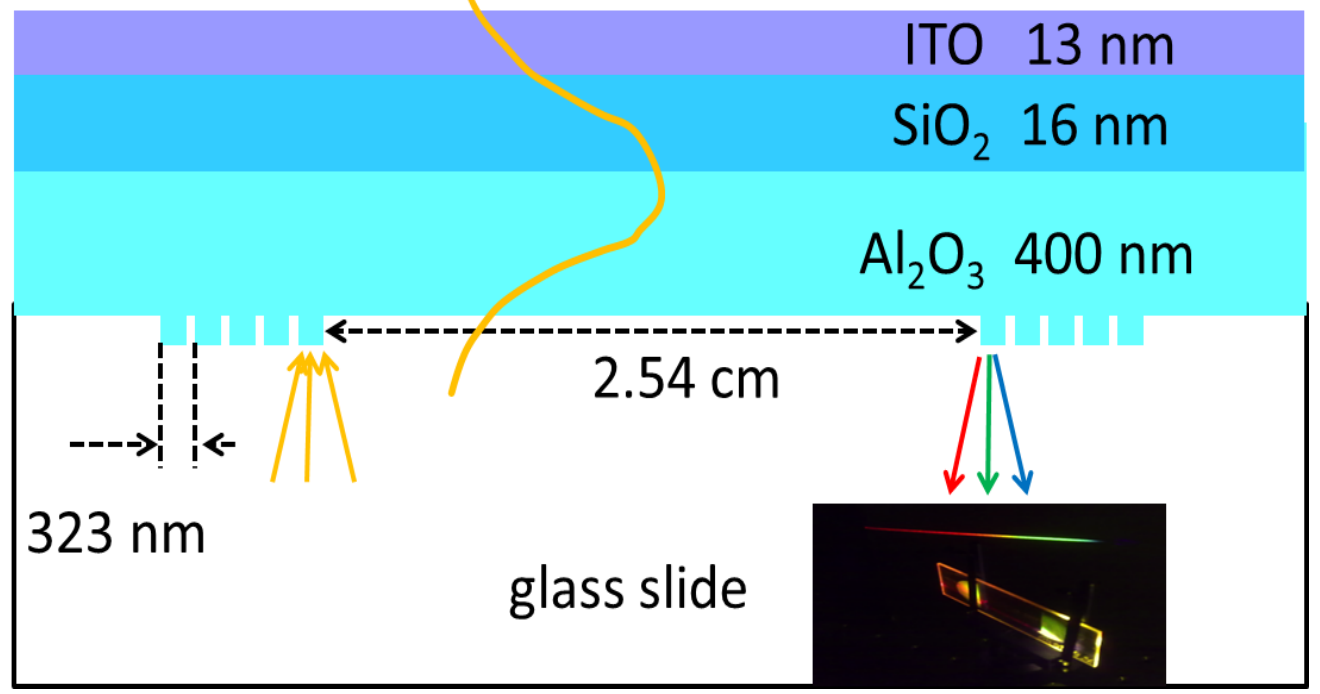

Figure 2-1: The architecture of an electro-active single-mode integrated optical waveguide (not in scale). Two surface relief gratings with $323 \mathrm{~nm}$ pitch size are used as the light couplers. The structure of the single-mode multi-layer waveguide is formed by a $400 \mathrm{~nm}$ aluminum oxide, a $16 \mathrm{~nm}$ silicon dioxide, and a $13 \mathrm{~nm}$ transparent conductive indium tin oxide. Different probing wavelengths can be coupled into the system at its own coupling angle. With $2.54 \mathrm{~cm}$ probing length, the guided light is coupled out at the right side grating. A real out-coupled spectrum is shown in the inset. 


\section{Deposition of Aluminum Oxide and Silicon Dioxide Layers}

A substrate with a pair of surface relief gratings was placed in the ALD coating chamber for a sufficiently long time (10 minutes) to reach the deposition temperature $\left(250^{\circ} \mathrm{C}\right)$ and high vacuum $(7.5 \mathrm{mbar})$. The precursors for the aluminum oxide deposition were Trimethylaluminum (TMA, $\left.\mathrm{Al}_{2}\left(\mathrm{CH}_{3}\right)_{6}\right)$ and $\mathrm{H}_{2} \mathrm{O}$. The pulse time was $150 \mathrm{~ms}$ for both of the precursors and the purging time was $1 \mathrm{~s}$ between the pulses. With the calibrated deposition rate, 3756 cycles were used for a $400 \mathrm{~nm}$ thickness of aluminum oxide. After the aluminum oxide layer deposition, enough purging time (approximately 10 minutes) was used to clean the delivery tubes and the deposition chamber. Then a silicon dioxide layer was deposited without breaking the vacuum. The pulse time of the two precursors was $50 \mathrm{~ms}$ for BisdiethylaminoSilane (BDMS, heated $30^{\circ} \mathrm{C}$ ) and $1.5 \mathrm{~s}$ for $\mathrm{O}_{3}$. For the purging time, 1 s was used between the two precursors. A total of 2000 cycles were used for the deposition of $16 \mathrm{~nm}$ of silicon dioxide layer.

The thickness of each layer was determined by matching the simulated transmittance spectrum to the one measured by a spectrophotometer (CARY 300). A Mathematica program using the transfer-matrix method to calculate the transmittance profile for a multi-layer structure (Appendix 3) was used. In Figure $2-2$, a match is observed between the simulation result and the measured transmittance. The fitting parameters for the alumina film were $n_{\mathrm{Al}_{2} \mathrm{O}_{3}}=$ 1.65 and $t_{\mathrm{Al}_{2} \mathrm{O}_{3}}=409 \mathrm{~nm}$, and for silicon dioxide were $n_{\mathrm{SiO}_{2}}=1.51$ and $t_{\mathrm{SiO}_{2}}=$ $16 \mathrm{~nm}$. 


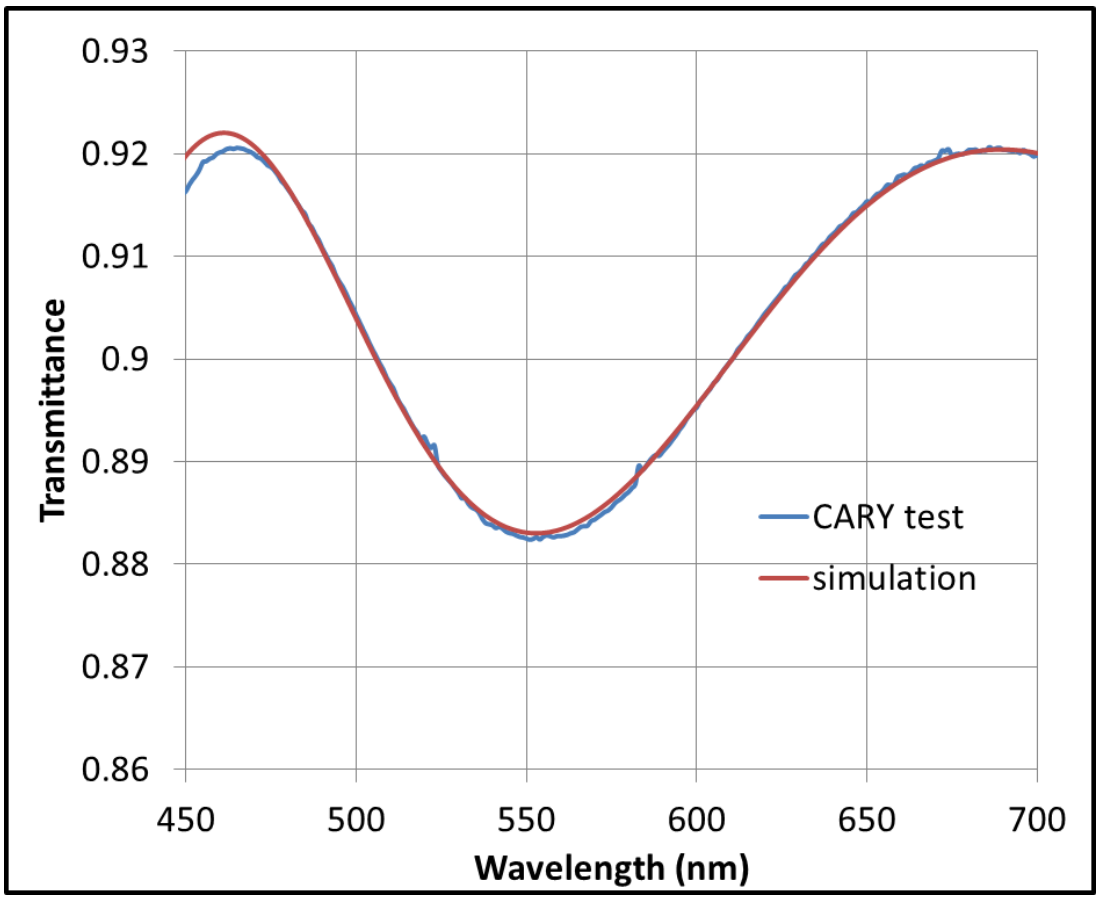

Figure 2-2: Match of the experimental and the simulated transmittance curves to determine the thickness of the aluminum oxide and the silicon dioxide layers. The fitting parameters for the alumina film are $n_{\mathrm{Al}_{2} \mathrm{O}_{3}}=1.65$ and $t_{\mathrm{Al}_{2} \mathrm{O}_{3}}=409 \mathrm{~nm}$, and for silicon dioxide were $n_{\mathrm{SiO}_{2}}=1.51$ and $t_{\mathrm{SiO}_{2}}=16 \mathrm{~nm}$.

\section{Indium Tin Oxide Deposition and Properties Characterization}

ITO is the most commonly used material for transparent conductive electrodes because of its low resistivity $\left(10^{-3}-10^{-4} \Omega \mathrm{cm}\right)$ and high transparency. Various methods can be used for ITO film deposition, such as chemical solution deposition [115], ALD [116], radio frequency sputtering [117-119] and DC magnetron sputtering [120]. Because of the superior controllability, the pulsed DC sputtering technique was selected [121]. By changing the deposition and annealing 
parameters, such as sputtering temperature, voltage [122], $\mathrm{Ar} / \mathrm{O}_{2}$ ratio [123-125], annealing atmosphere (in air or $\mathrm{N}_{2}$ ) [126], and annealing temperature [127], the optical and electrical properties of the ITO film can be adjusted to specific requirements. A SM-IOW requires several centimeters of beam propagation inside the guided structure to increase the detection sensitivity, and an extremely low extinction coefficient of the ITO film is required, while the resistivity of the ITO film should be as low as possible to support electrochemical potential modulations. For the characterization process, varied $\mathrm{O}_{2}$ flow rate during the sputtering was tested, while the other fabrication parameters were kept constant. The same inert annealing in the $\mathrm{N}_{2}$ atmosphere was applied. We worked with relatively thick ITO films (approximately $400 \mathrm{~nm}$ ) to obtain the optical properties. The most suitable $\mathrm{O}_{2}$ flow rate was selected to coat an ultra-thin layer (approximately $13 \mathrm{~nm}$ ) of ITO for an EA-SM-IOW device.

\section{Pulsed DC sputtering}

Pulsed DC sputtering technique was used to coat an ITO film on cleaned glass slides at room temperature. The ITO target was $3 \mathrm{inch}, 99.99 \%$ purity with $\left(\operatorname{In}_{2} \mathrm{O}_{3}\right)$ 90\% (SnO) $10 \%$ by weight. The sputtering power was 200 Watt at $20 \mathrm{KHz}$ with $1 \mu \mathrm{s}$ pulse. The flow rate of Ar was fixed at $12 \mathrm{sccm}$. The rotation of the substrate holder was $20 \mathrm{rpm}$. Sputtering time was 30 minutes for each sample to form an ITO film with a thickness of approximately $400 \mathrm{~nm}$. Various $\mathrm{O}_{2}$ flow rates from $0.4 \mathrm{sccm}$ to 0.8 sccm were tested. 
Transmittance spectra of these thick ITO samples as sputtered were measured by a spectrophotometer, as shown in Figure 2-3. As expected, for a higher $\mathrm{O}_{2}$ flow rate, the film had a higher transmittance. At $0.7 \mathrm{sccm}$ and $0.8 \mathrm{sccm} \mathrm{O}_{2}$ flow rate, the transparency of the ITO films reached saturation.

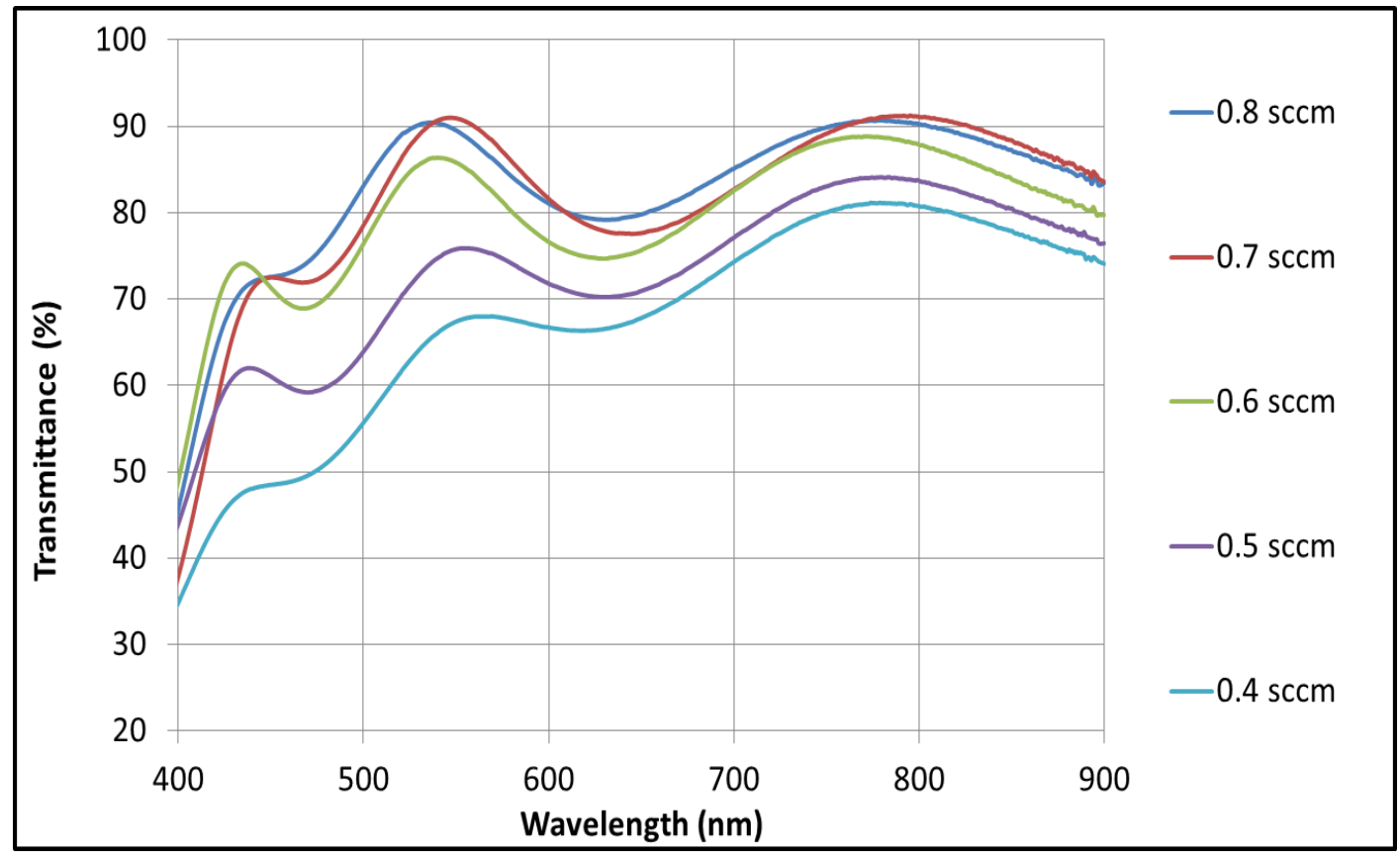

Figure 2-3: Transmittance curves for ITO films as deposited under various $\mathrm{O}_{2}$ flow rate. The higher the $\mathrm{O}_{2}$ flow rate, the higher the transmittance is. At $0.7 \mathrm{sccm}$ and 0.8 sccm, the transmittance reaches saturation.

Inert $\mathrm{N}_{2}$ annealing

To achieve lower resistivity and better transparency in the visible spectral range for the ITO film, an inert annealing in $\mathrm{N}_{2}$ atmosphere at $250{ }^{\circ} \mathrm{C}$ for 10 minutes 
(1 hour for temperature ramping up and 1 hour for temperature ramping down) was used [124]. An inert annealing can activate tin element, create more 0 vacancies, reduce grain boundaries, and change the ITO film from amorphous to a polycrystalline structure. These effects contribute to the conductivity by increasing the mobility and reducing the scattering. Figure 2-4 shows the transmittance curves of ITO films as sputtered with $0.6 \mathrm{sccm} \mathrm{O}_{2}$ flow rate and after the inert annealing. The ITO film is clearly more transparent in the visible range after the inert annealing.

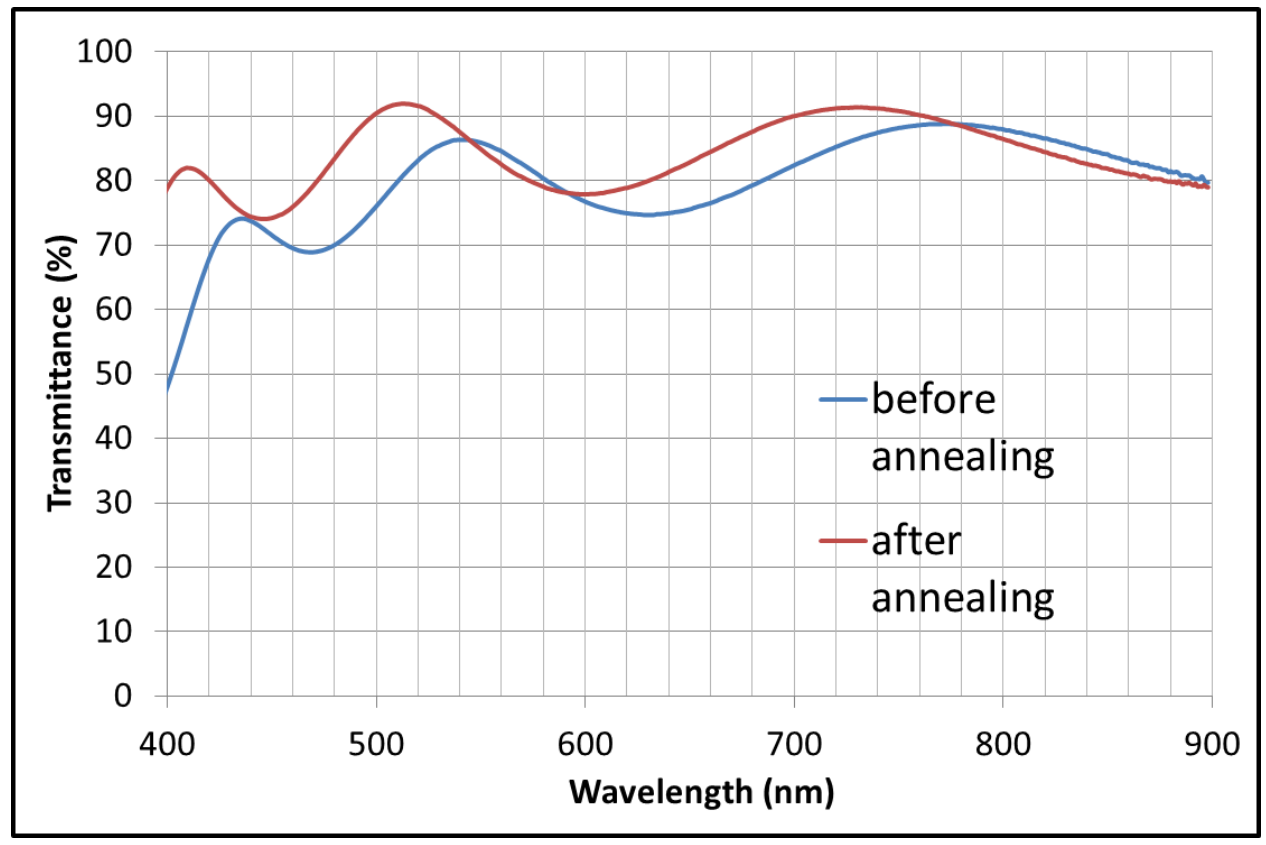

Figure 2-4: Transmittance curves for ITO films as deposited with $0.6 \mathrm{sccm} \mathrm{O}_{2}$ flow rate and after the inert annealing process. After the annealing process, the film is more transparent in the visible range. 
By using the information (wavelength and intensity) from the interference peaks and dips of the transmittance curves, the thickness, the real part of the refractive index, and the extinction coefficient (the imaginary part of the refractive index, $k_{I T O}$ ) could be calculated for the ITO samples [128]. The extinction coefficient around $550 \mathrm{~nm}$ is plotted against $\mathrm{O}_{2}$ flow rate in Figure 2-5. Before the inert annealing, a higher $\mathrm{O}_{2}$ flow rate resulted in a lower extinction coefficient. As the $\mathrm{O}_{2}$ flow rate kept increasing, the extinction coefficient reached minimum value. After the inert annealing, the transparency was substantially improved, especially for the samples with low $\mathrm{O}_{2}$ flow rate. For the films sputtered at high $\mathrm{O}_{2}$ flow rate, the inert annealing did not provide any significant improvement in the film transparency. The film with $0.6 \mathrm{sccm} \mathrm{O}_{2}$ flow rate had the lowest extinction coefficient, $1.7 \times 10^{-4}$, after the inert annealing.

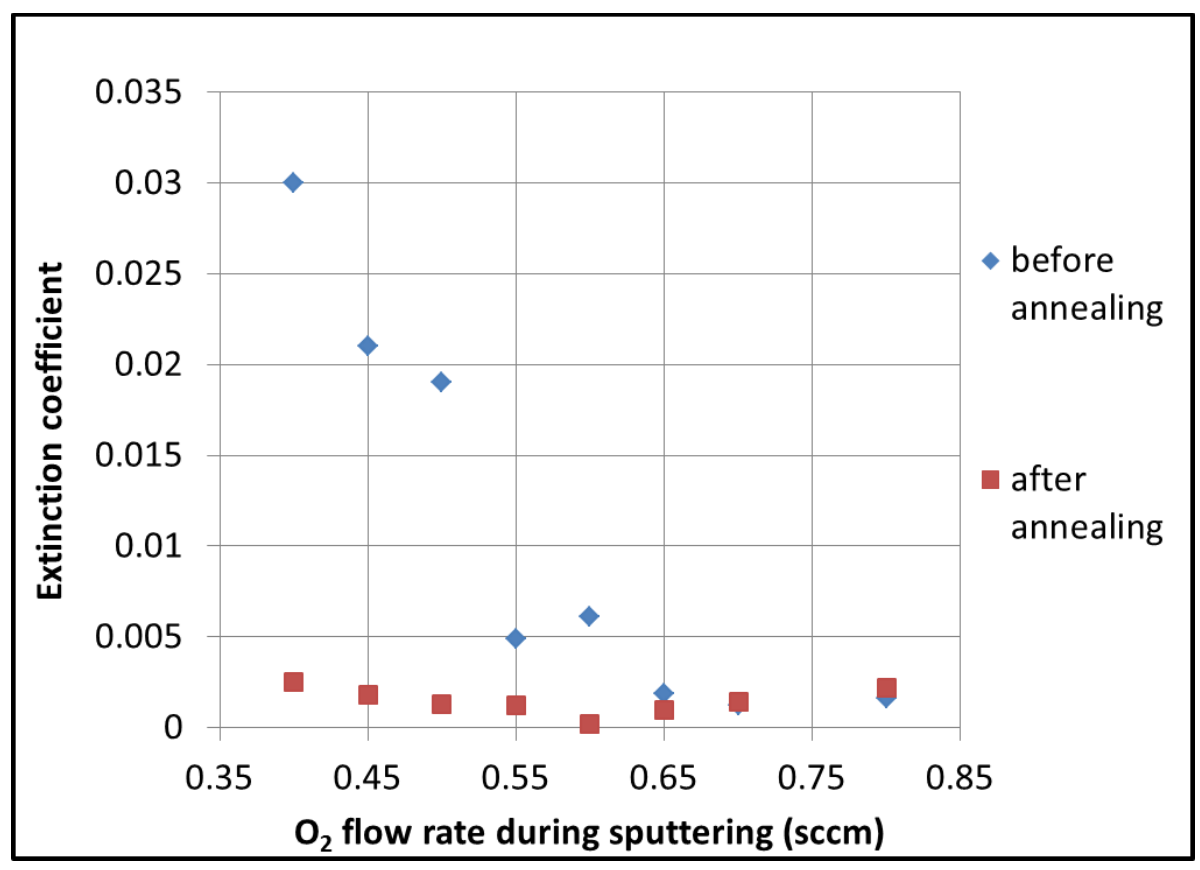


Figure 2-5: Extinction coefficient of the ITO films as sputtered and after the inert annealing process at various $\mathrm{O}_{2}$ flow rate during sputtering deposition. At $0.6 \mathrm{sccm}$, the lowest extinction coefficient is observed for the ITO film after the inert annealing process.

The sheet resistance and the thickness of the film were used to calculate the resistivity with Equation 2-2. The resistivity of the films before and after the inert annealing is shown in Figure 2-6.

$$
\rho=R_{\mathbf{m}} t
$$

Before the inert annealing, the resistivity had a minimum value between 0.5 and $0.6 \mathrm{sccm} \mathrm{O}_{2}$ flow rates. When the $\mathrm{O}_{2}$ flow rate was low, the $\mathrm{Sn}$ element could not be activated at the room temperature and resulted in samples with high resistivity. When the $\mathrm{O}_{2}$ flow rate was high, the stoichiometry of the film was close to a fully oxide and also showed high resistivity. After the inert annealing, the Sn element was activated and a lower resistivity was achieved for all tested $\mathrm{O}_{2}$ flow rates.

At $0.6 \mathrm{sccm} \mathrm{O}_{2}$ flow rate, the lowest optical extinction coefficient and an acceptable electrical resistivity were achieved. This particular condition $(0.6 \mathrm{sccm}$ $\mathrm{O}_{2}$ flow rate) was chosen to coat an ultra-thin ITO film on waveguide samples for the spectroelectrochemistry applications. 


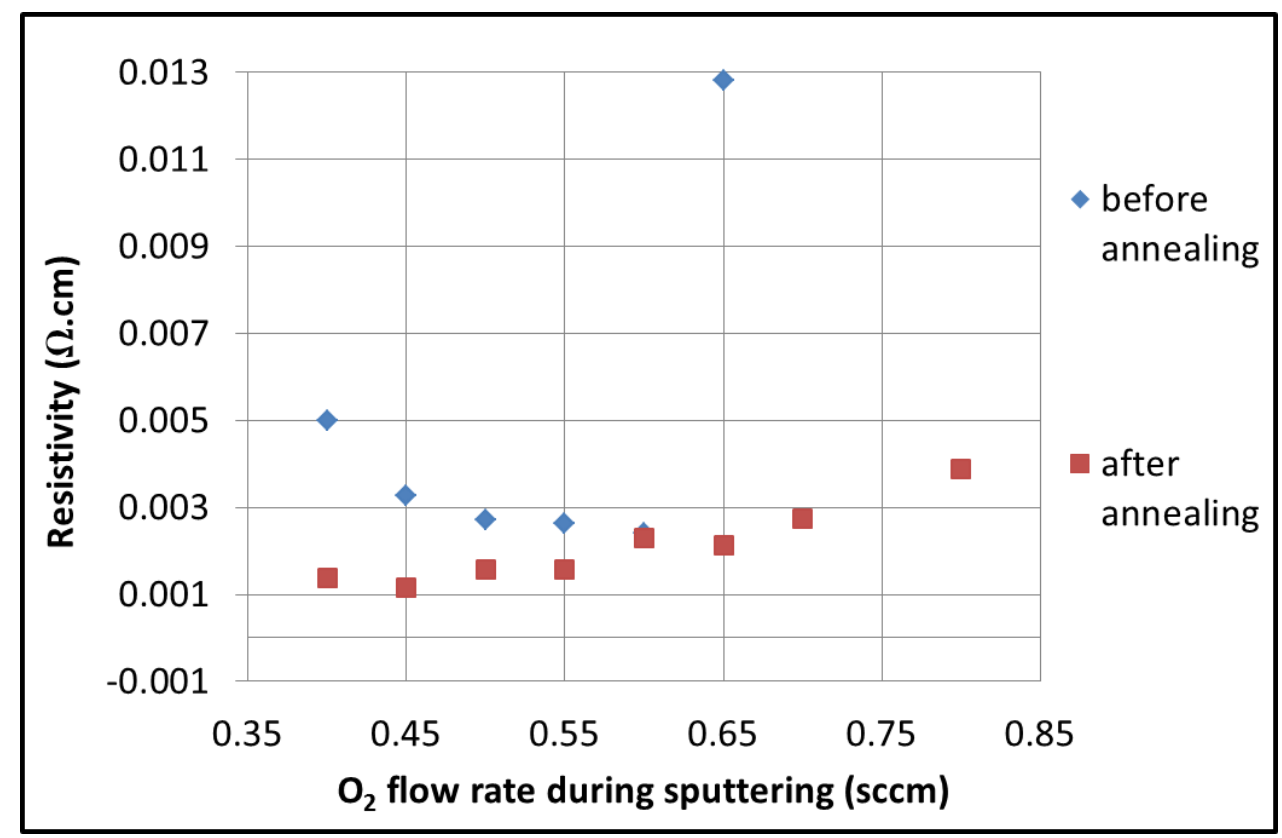

Figure 2-6: Resistivity of the ITO films as sputtered and after the inert annealing process with various $\mathrm{O}_{2}$ flow rate during sputtering deposition. A lower $\mathrm{O}_{2}$ flow rate during the sputtering resulted in a lower resistivity after the inert annealing process.

For ITO films sputtered with $0.6 \mathrm{sccm} \mathrm{O}_{2}$ flow rate, the real refractive indices before and after the inert annealing were plotted against the wavelength, as shown in Figure 2-7. After the inert annealing, the real refractive index of the ITO sample was lower, and they were used in the effective refractive index calculation in section 2.2.1. 


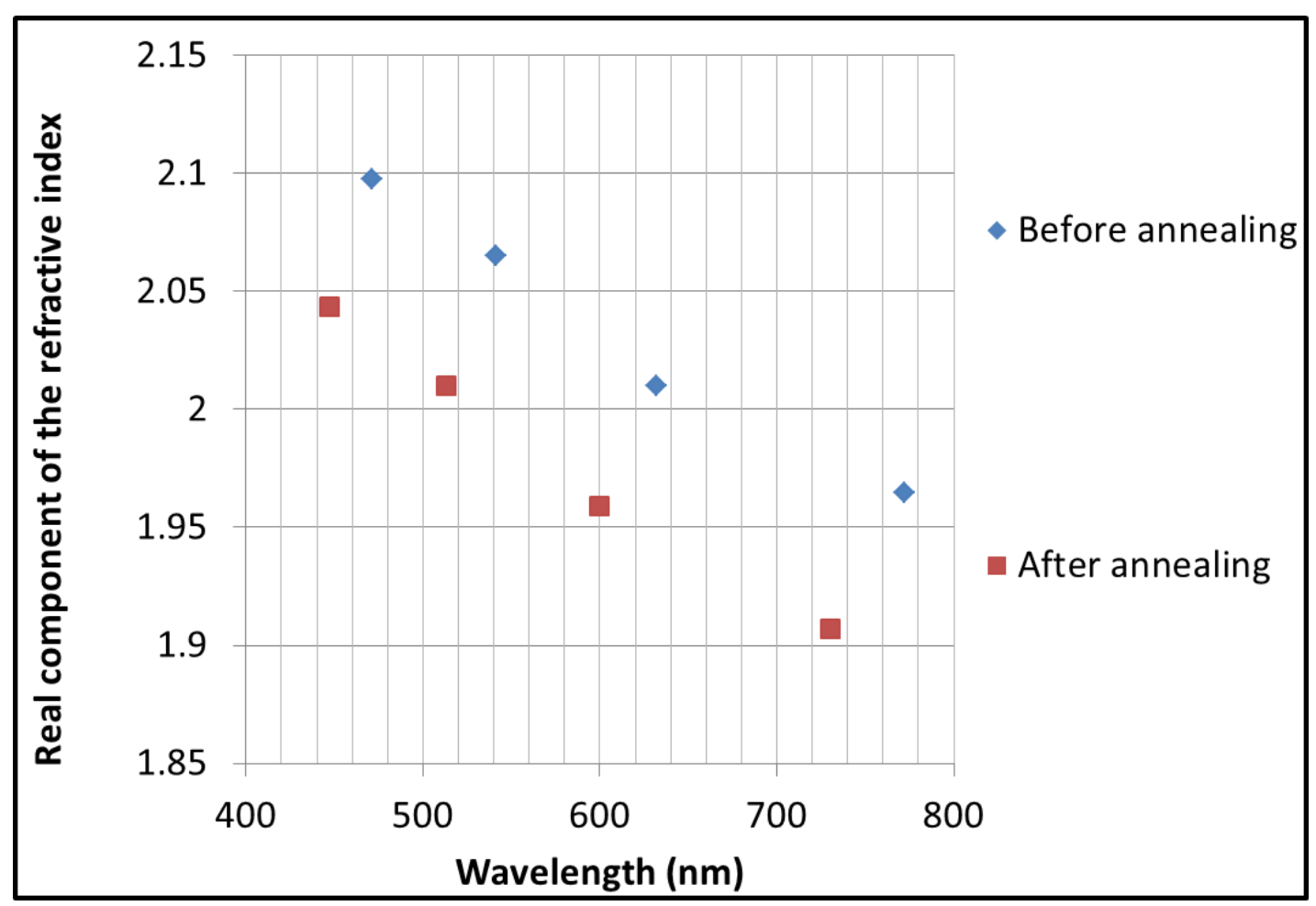

Figure 2-7: Real refractive index of thick ITO films before and after the inert annealing with $0.6 \mathrm{sccm} \mathrm{O}_{2}$ flow rate during the sputtering. Lower real refractive index after the inert annealing was observed.

Optimization of the ultra-thin ITO film

At $0.6 \mathrm{sccm} \mathrm{O}_{2}$ flow rate, ultra-thin ITO films (approximately $8 \mathrm{~nm}, 13 \mathrm{~nm}$ or $18 \mathrm{~nm}$ ) were sputtered on top of SM-IOW slides. The sputtering time was determined by the calibrated sputtering rate. As it has been reported, the physical properties of ITO thin-films are also dependent on the thickness [129-131]. After the same inert annealing, the resistivity of an ultra-thin ITO film dropped dramatically, but the extinction coefficient did not decline as observed with the 
thicker ITO samples. Propagation loss measurement, displayed in Figure 2-8, was used to examine the intensity of the out-coupled light. A $543 \mathrm{~nm}$ laser beam was used. The coupling angle was selected to achieve the best coupling condition. The incident, transmitted, reflected and out-coupled beams from the front and back sides of the EA-SM-IOW were measured by a power meter.

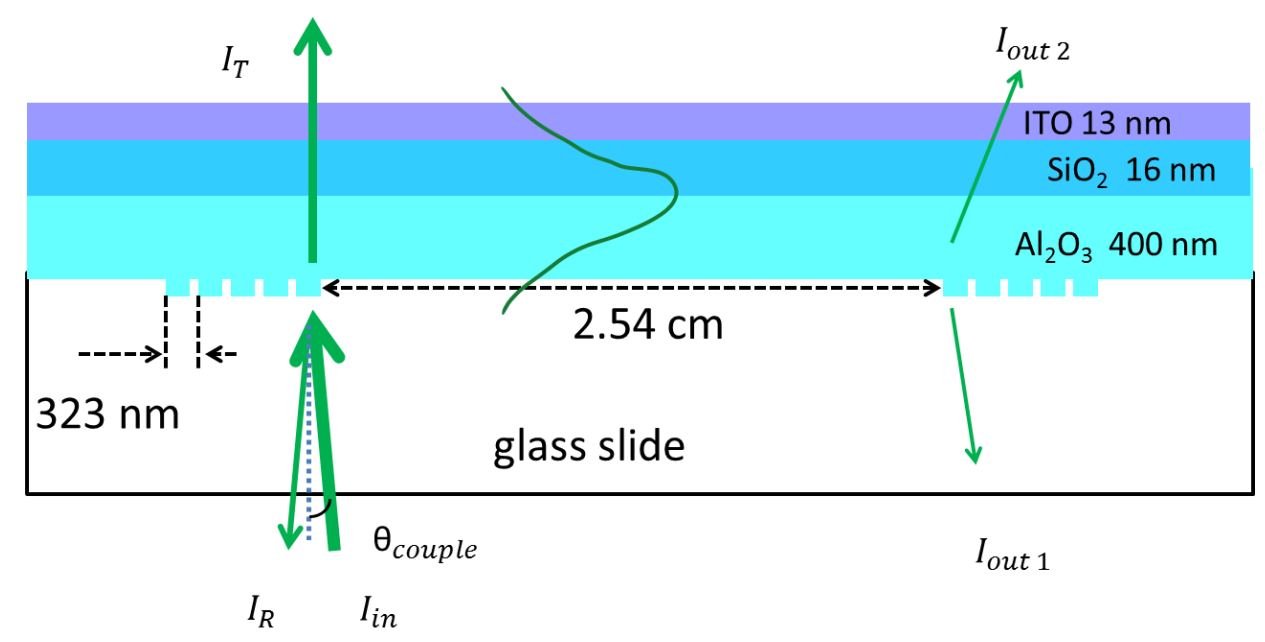

Figure 2-8: Demonstration of the propagation loss and the coupling efficiency measurement set-up.

The propagation loss and the coupling efficiency (C.E.) can be calculated with Equation 2-3 and Equation 2-4, where $I_{\text {in }}$ is the incident beam intensity, $I_{R}$ is the reflected beam intensity, $I_{T}$ is the transmitted beam intensity, $I_{\text {out } 1}$ and $I_{\text {out } 2}$ are the two out-coupled light beams intensities. Without any further optimization, the sensor with a $13 \mathrm{~nm}$ thick ITO film had a $12 \mathrm{~dB} / \mathrm{cm}$ propagation loss with approximately $35 \%$ coupling efficiency. 


$$
\begin{aligned}
& C . E .=\frac{I_{\text {in }}-I_{R}-I_{T}}{I_{\text {in }}} 100 \% \\
& \text { Loss }=-10 \log _{10} \frac{I_{\text {out } 1}+I_{\text {out } 2}}{I_{\text {in }}-I_{R}-I_{T}}
\end{aligned}
$$

An active annealing process in room atmosphere, containing $\mathrm{O}_{2}$, was used to increase the transparency of an ultra-thin ITO layer in the visible range. $\mathrm{O}_{2}$ diffused into the ultra-thin ITO layer to reduce 0 vacancies. The fewer defects in the film, the lower residual absorption and the lower propagation loss were achieved. This active annealing process always started at $150{ }^{\circ} \mathrm{C}$ (lower than $\mathrm{N}_{2}$ annealing) to avoid a fast reaction rate. Temperature was raised up with $50{ }^{\circ} \mathrm{C}$ increments to achieve a medium reaction speed if it was too slow at $150^{\circ} \mathrm{C}$. The sample was heated until an acceptable low propagation loss was achieved, approximately $6 \mathrm{~dB} / \mathrm{cm}$.

The resistivity of the ultra-thin ITO film after the active annealing was also examined to make sure a sufficient conductivity could be applied for the potential modulation experiments. The sheet resistance of the final device was approximately $2 \mathrm{~K} \Omega$. The resulted resistivity, $2.6 \times 10^{-3} \Omega \mathrm{cm}$, was only slightly higher than the characterized result, $2.3 \times 10^{-3} \Omega \mathrm{cm}$ from section 2.4 .2 after the inert annealing for thicker ITO samples.

The same Mathematica program was used to simulate the transmittance profiles of the multilayer structure with an optimized ultra-thin ITO layer. The refractive index and the thickness for the aluminum oxide and the silicon dioxide films were kept the same for the simulation. Matches between the measurement and 
the simulation are shown in Figure 2-9. The fitting parameters for the ITO film were $n_{\text {ITO }}=1.88$ and $t_{\text {ITO }}=18 \mathrm{~nm}$.

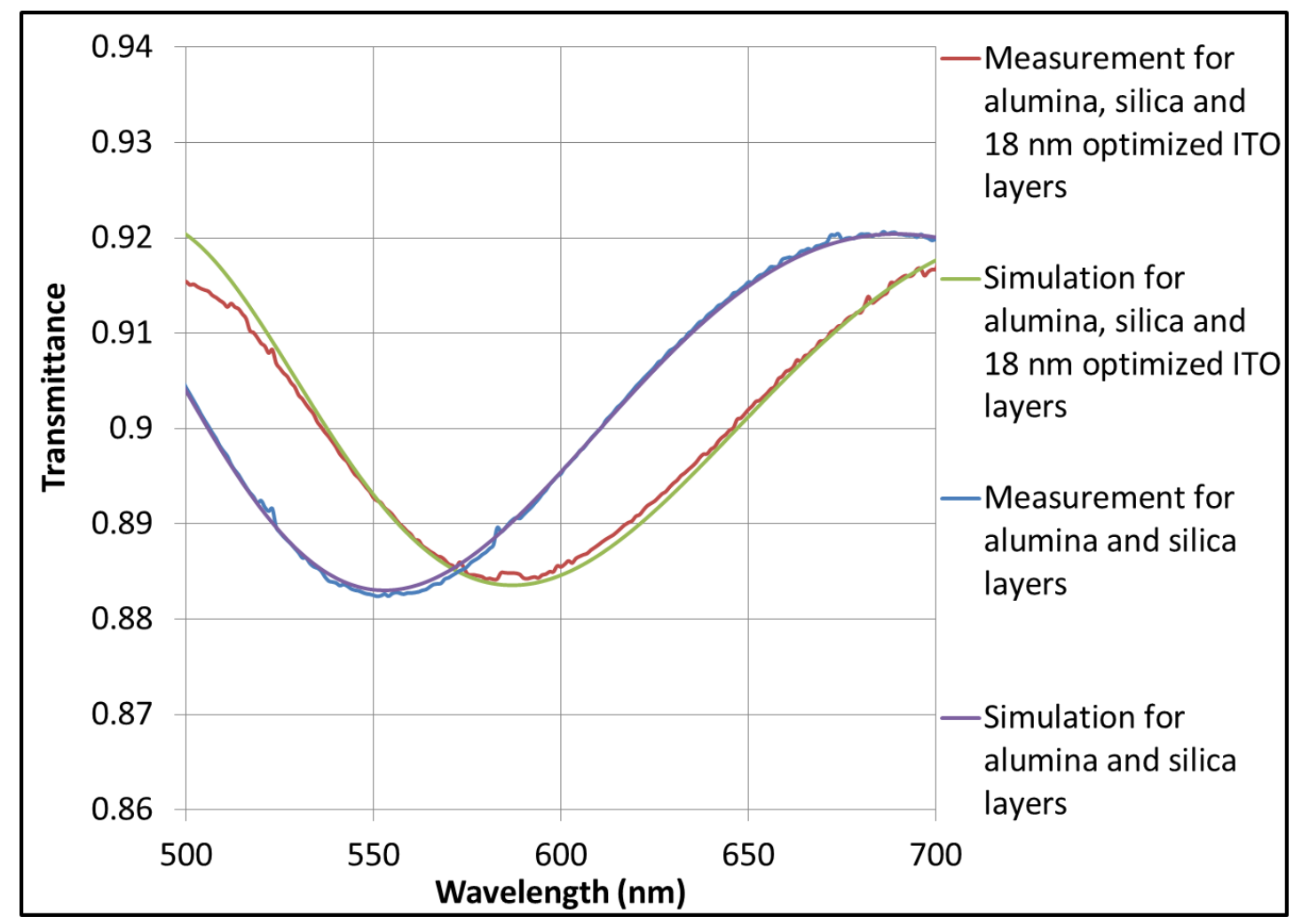

Figure 2-9: Simulations of transmittance profile to determine the thickness of the ultra-thin ITO film. The fitting parameters for the alumina film are $n_{\mathrm{Al}_{2} \mathrm{O}_{3}}=$ 1.65 and $t_{\mathrm{Al}_{2} \mathrm{O}_{3}}=409 \mathrm{~nm}$, for silicon dioxide were $n_{\mathrm{SiO}_{2}}=1.51$ and $t_{\mathrm{SiO}_{2}}=16 \mathrm{~nm}$, and for the ITO film were $n_{\text {ITO }}=1.88$ and $t_{\text {ITO }}=18 \mathrm{~nm}$. Matches were observed between the simulation and the experimental results.

According to the experimental observations, the optical and electrical properties of an ultra-thin ITO film can be tuned by the inert and active annealing 
processes. Active annealing increases the optical transparency and the inert annealing increases the electrical conductivity. Although these annealing processes can be used to improve the properties of the ultra-thin ITO film for spectroelectrochemical applications, the quality of the as sputtered ITO film determines how far these properties can be modified.

\section{Device Performance Characterization}

The performance of an optimized device was characterized. The following results are from an EA-SM-IOW device with an approximately $13 \mathrm{~nm}$ ultra-thin ITO layer.

\section{Broadband coupling}

The strategy to couple a broadband incident light into the single-mode waveguide has been described in various literatures [132-134]. The schematic of the experimental set-up is shown in Figure 2-10. For the optical system, white light from a tungsten-halogen lamp was focused into a fiber $(600 \mu \mathrm{m}$ core diameter, multi-mode), and connected to a light coupling system. This system included a lens to collimate the light beam, a polarizer for polarization selection, and a pair of cylindrical lenses to focus the light into a line along the direction of the grating stripes at the edge of the grating coupler. The wavelengths, approximately from 500 $\mathrm{nm}$ to $600 \mathrm{~nm}$, could be coupled into the waveguide based on this set-up. A similar arrangement of optical elements was used to collect the light out of the device and couple the signal into an optical fiber. This coupling set-up is referred to as the 
stationary coupling set-up. For the detection, the optical signal was collected by a monochromator (Princeton, 2358) which was connected with an intensified CCD (ICCD, Princeton, PI-MAX 3) camera.

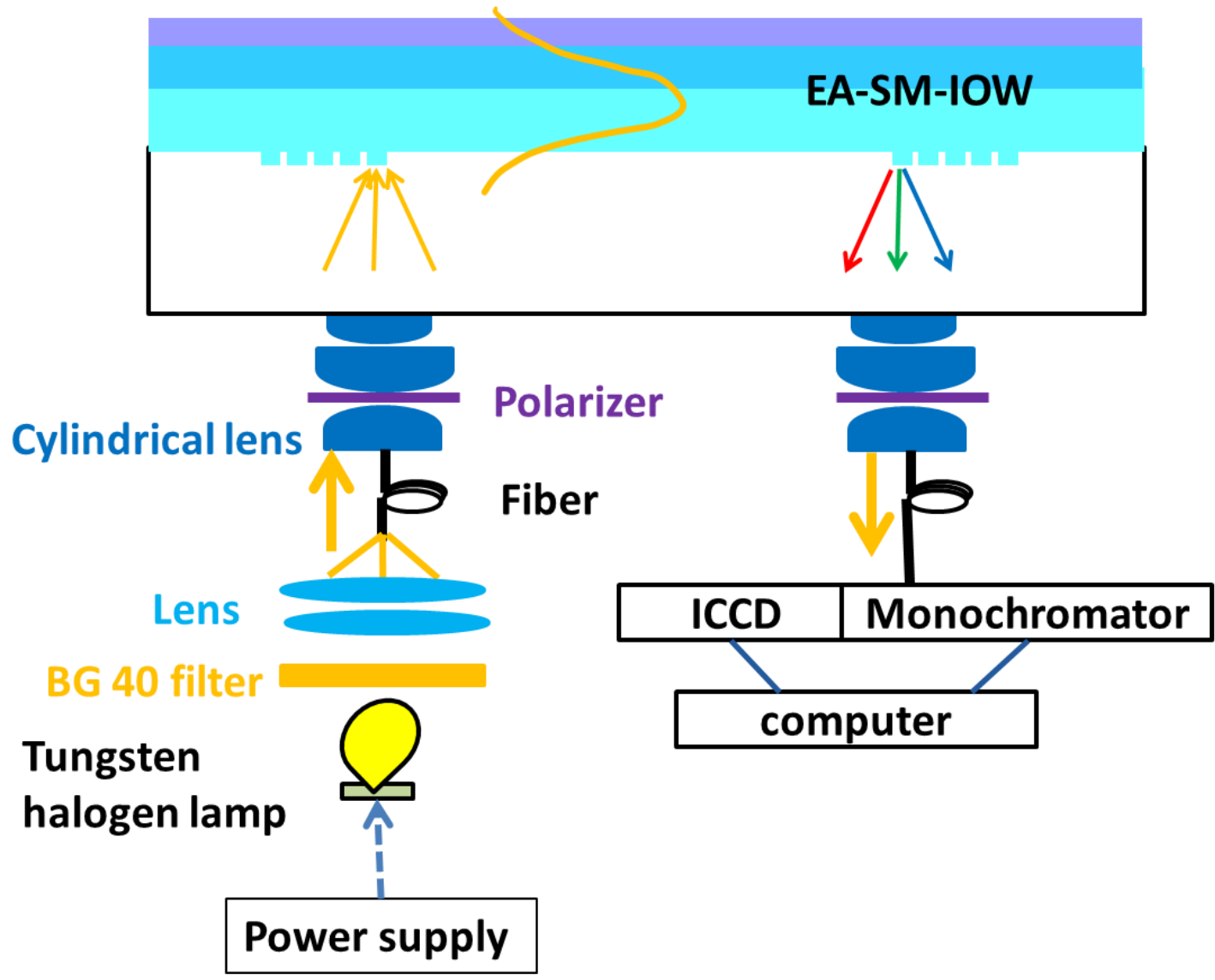

Figure 2-10: Schematic representation of the experimental set-up for broadband light coupling. Two identical sets of optical elements are used to couple light into and out of the EA-SM-IOW. For the signal detection, an ICCD connected with a monochromator is used. 
The power supply for the tungsten-halogen lamp was set at $0.6 \mathrm{~V}$. The exposure time for the ICCD camera was $1 \mathrm{~s}$. A throughput signal in air was collected first (blue curve in Figure 2-11). This signal allowed an evaluation of the broadband spectrum coupling for the EA-SM-IOW. A strongly absorbing material (a black mark) was placed on the surface of the EA-SM-IOW between the two gratings to completely absorb the guided light. This signal is called the dark signal (red curve in Figure 2-11), including the stray light and the dark current signal from the camera. With this measurement, the throughput signal was assured truly from the guided light.

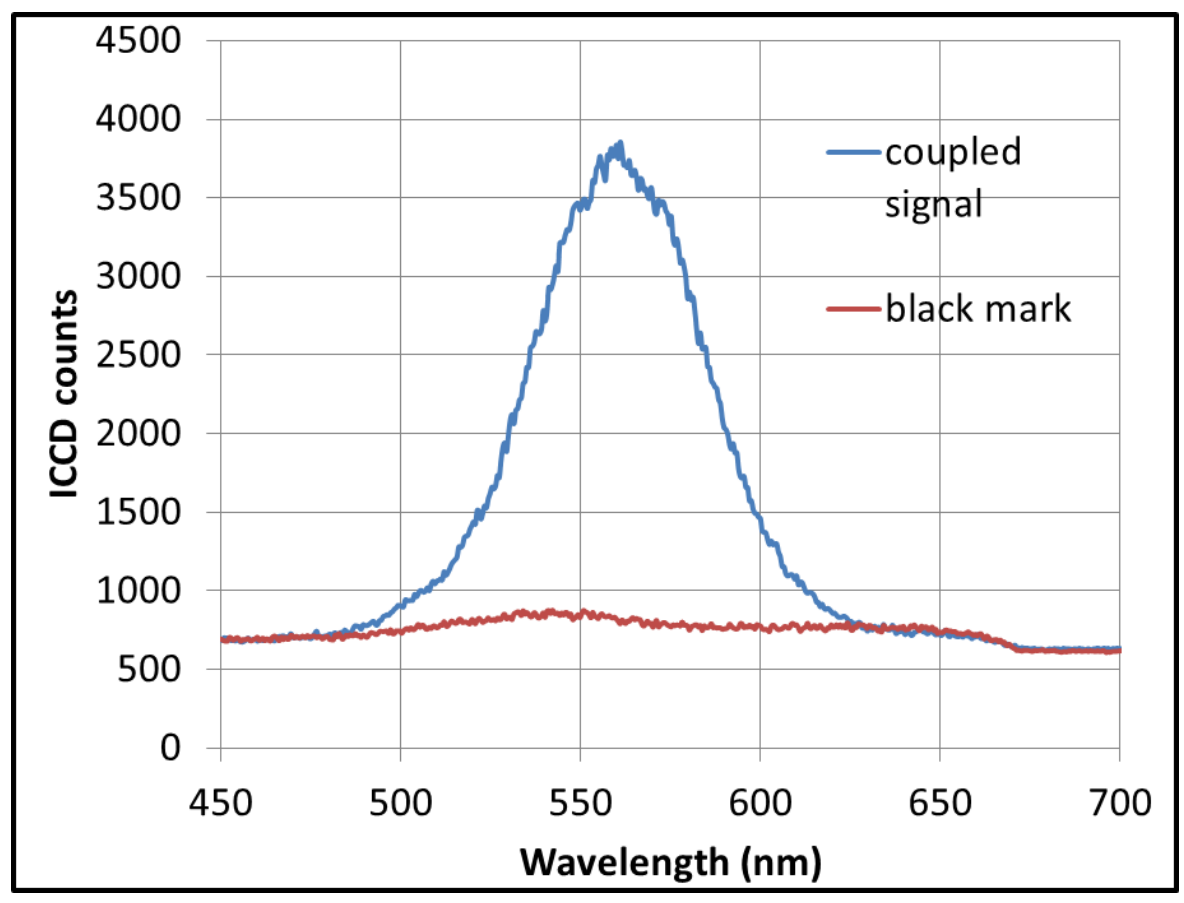

Figure 2-11: Broadband coupling for an EA-SM-IOW by using a tungsten-halogen lamp. A dark signal is plotted as the red curve. 
Effective refractive index calculation

By using the film thickness and the real component of the refractive index of each layer in an EA-SM-IOW, the effective refractive index of a fabricated device in an aqueous environment was calculated (Appendix 1). In Figure 2-12, this parameter is plotted against wavelength for the TE polarized light.

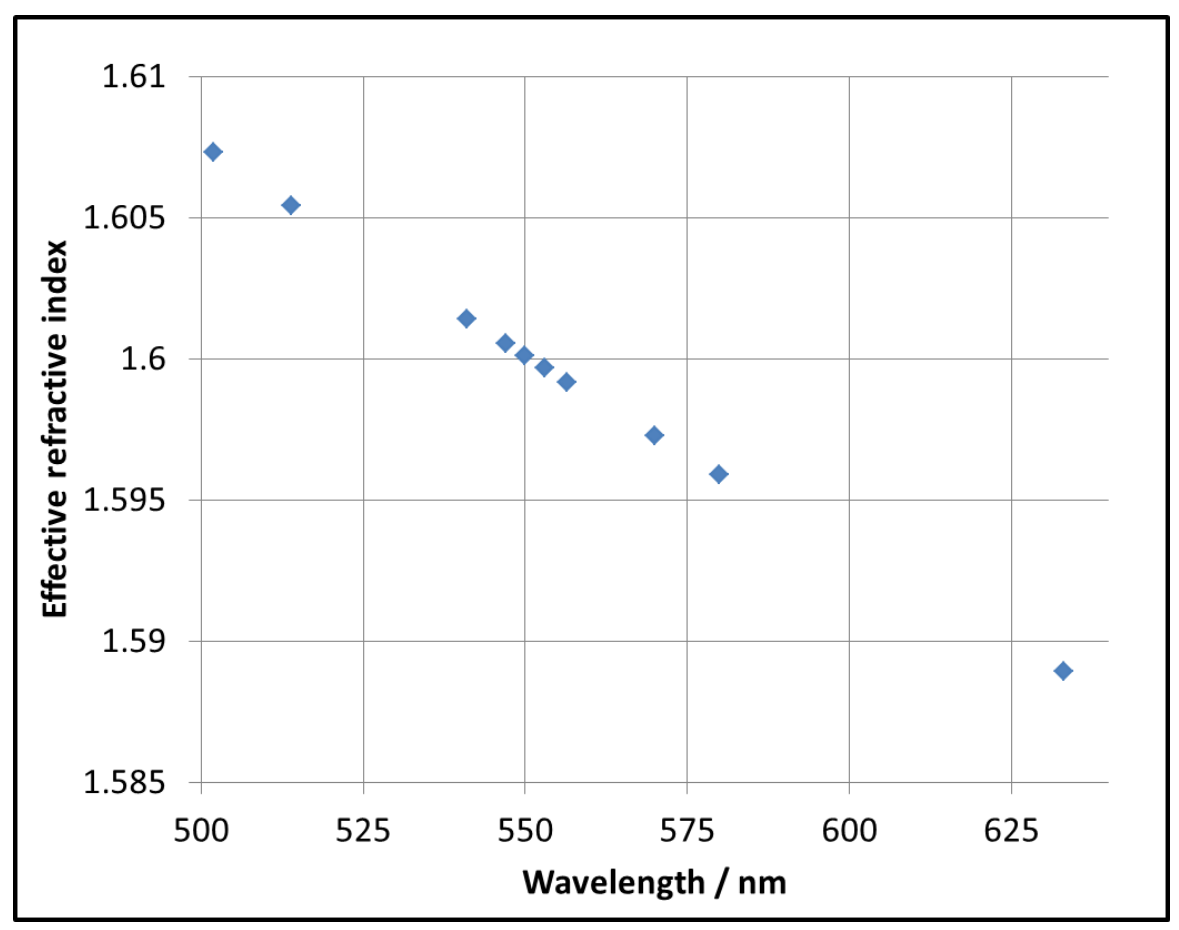

Figure 2-12: Simulation results of the effective refractive index of the device in an aqueous environment. 
Electric field distribution in the cross section of the EA-SM-IOW

A Mathematica program, using a transfer-matrix technique, was used to calculate the electric field distribution across the multi-layer structure of an EA-SM-IOW (Appendix 1) for an incident light at TE polarization, as shown in Figure 2-13 for wavelength $550 \mathrm{~nm}$. The same parameters for the effective refractive index simulation were used. The interface of the aluminum oxide layer and the glass slide is located at $z=0$. The red bar represents the layer of the ultra- thin ITO film (13 $\mathrm{nm})$, and the electric field intensity of this layer can be treated as a constant.

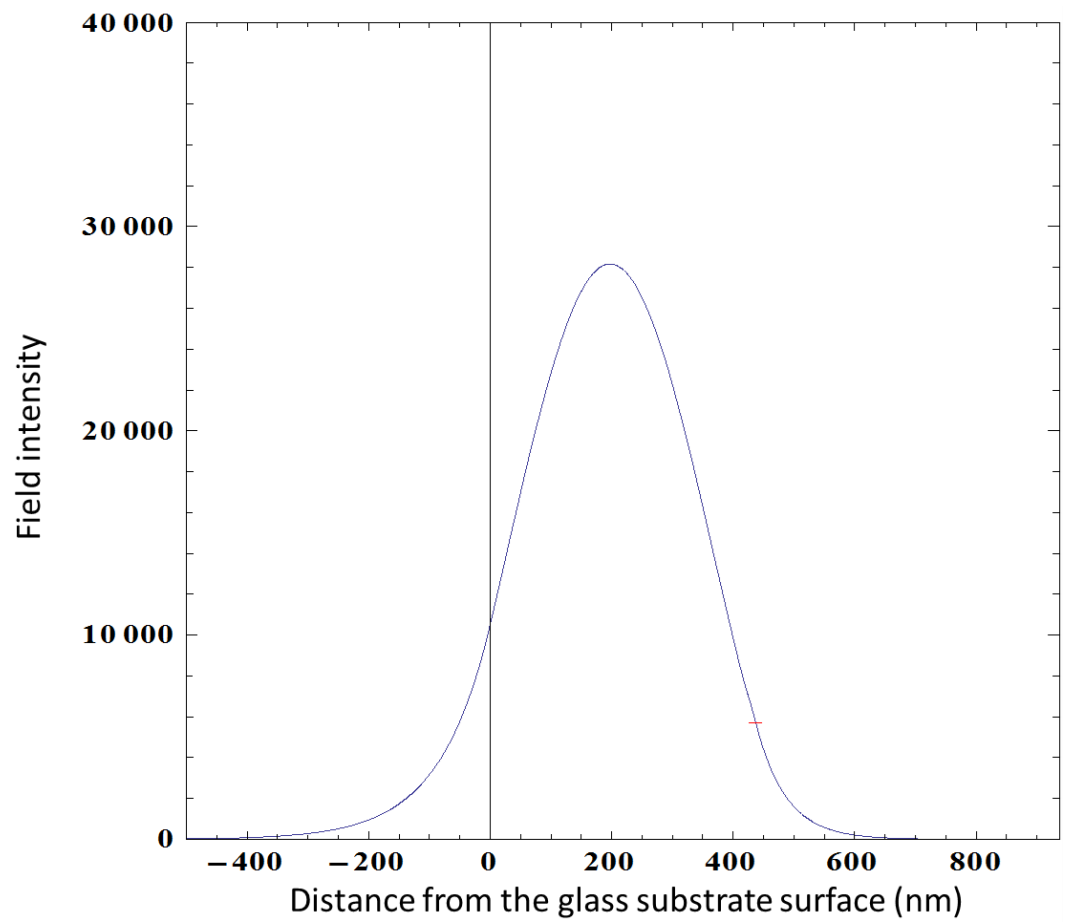

Figure 2-13: Simulation result of the field distribution of the device in the cross section. The interface of the alumina/glass slide locates at $z=0$, and red bar represents the layer of the $13 \mathrm{~nm}$ ITO film. 


\section{Sensitivity calculation}

Sensitivity, $S$, is an extremely important factor for the performance of the device and analysis of spectroelectrochemical experimental data. The definition of the sensitivity $[135,136]$ is given by Equation 2-5 For the same surface immobilized molecular thin film, the absorbance measured by an EA-SM-IOW probing structure is $A b s_{I O W, f i l m}$, as in Figure 2-14 (a), and the absorbance measured by the transmission probing structure is $A b s_{T, \text { film }}$, as in Figure 2-14 (b).

$$
S=\frac{A b s_{I O W, f i l m}}{A b s_{T, f i l m}}
$$

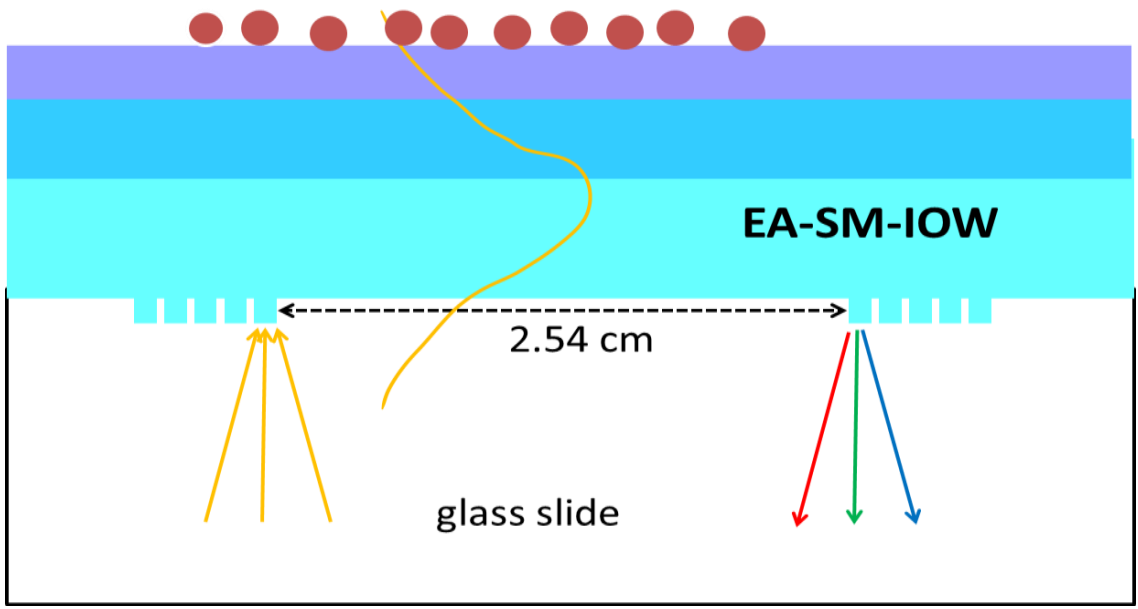

(a) 


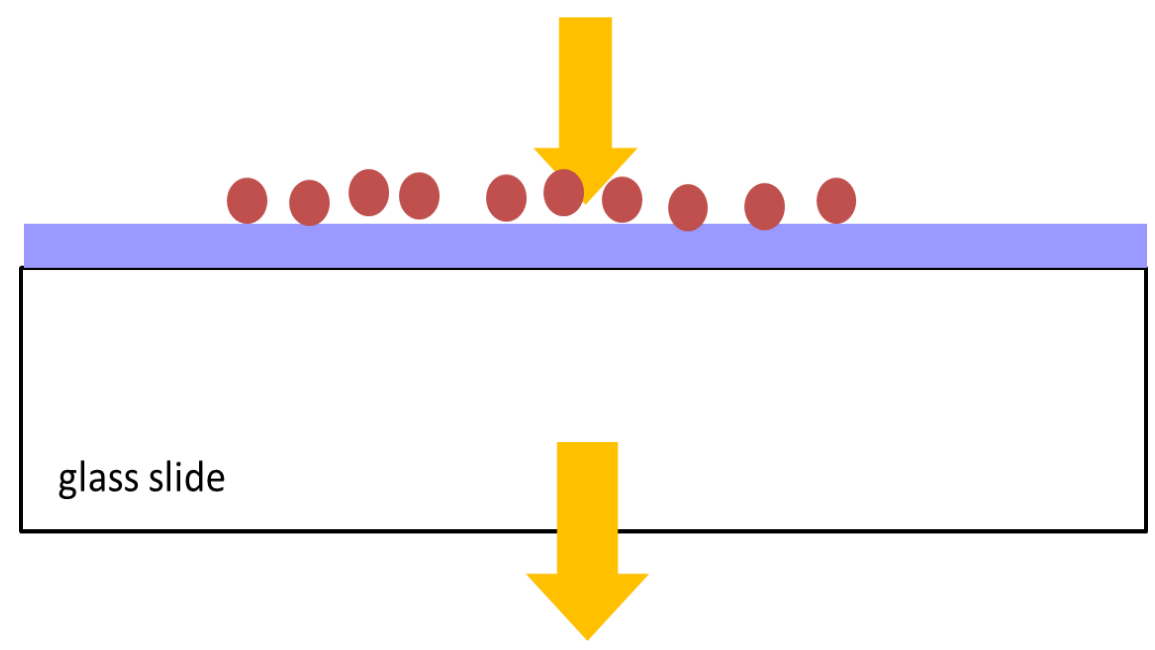

Figure 2-14: Absorbance measurement of a molecular thin film with (a) IOW probing structure and (b) transmittance probing structure.

Based on the strong confinement of the propagating light by the single-mode waveguide and a long probing distance (approximately $\mathrm{cm}$ ), the interaction of the probing light and the molecular thin film is enhanced for the IOW structure. With the transmittance measurement, although the probing light has the whole profile of the field distribution, the interaction between the probing light and molecules is limited by the thickness of the molecular thin film (approximately $\mathrm{nm}$ ). According to the definition, a Mathematica program was used (Appendix 1) to calculate the sensitivity factor. As shown in Figure 2-15 for a $2.54 \mathrm{~cm}$ propagation length, the sensitivity was more than 14,000 for the IOW structure. 


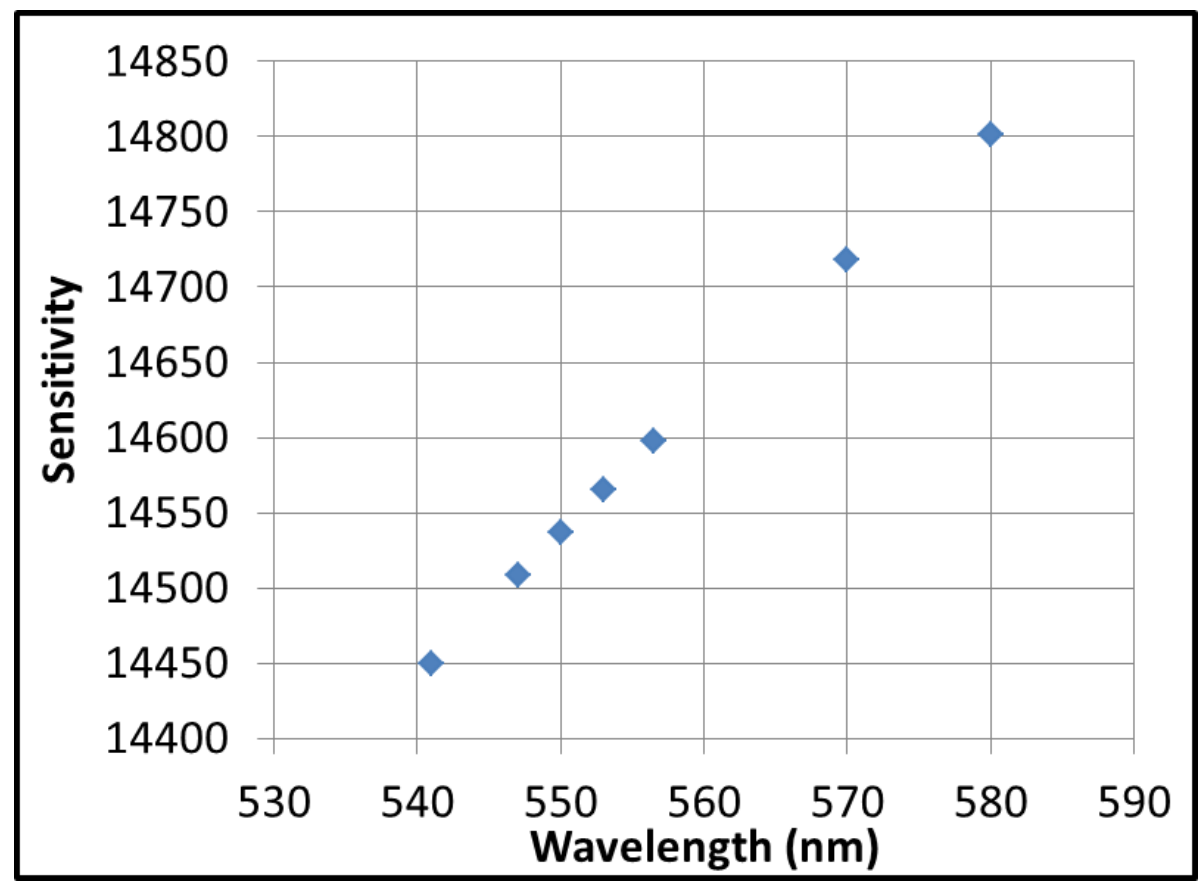

Figure 2-15: Plot of Sensitivity against probing wavelength at TE polarization. For this simulation, the same parameters of the EA-SM-IOW used for the effective refractive index and electrical field distribution simulations were applied.

Experimental determination of the sensitivity factor

The sensitivity factor was experimentally measured with blue dextran. Because blue dextran molecules do not adsorb on the surface of the device, its surface concentration is the same as the bulk concentration. The structures of blue dye and dextran unit are shown in Figure 2-16 (a) and (b). 


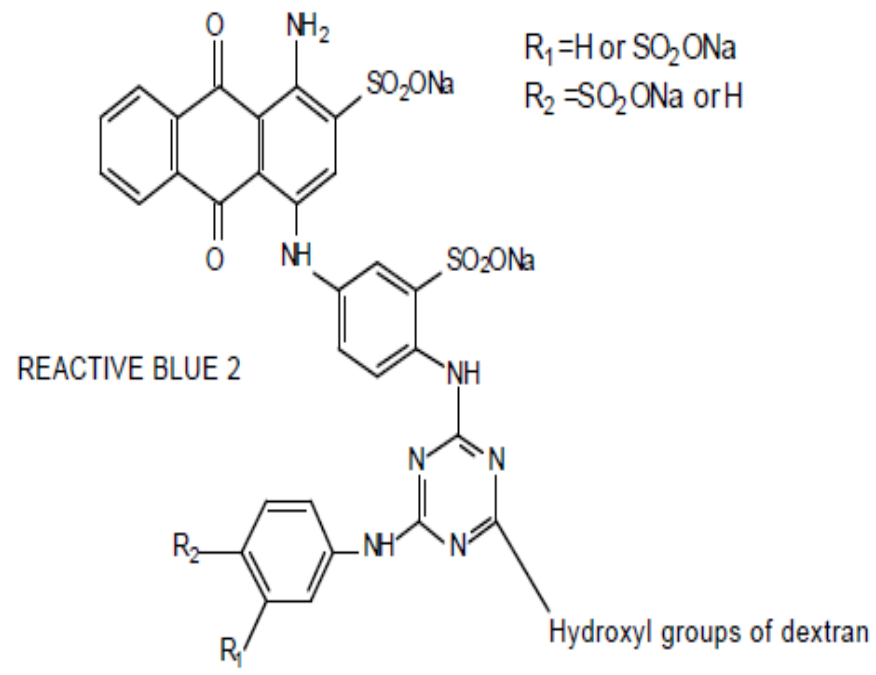

(a)

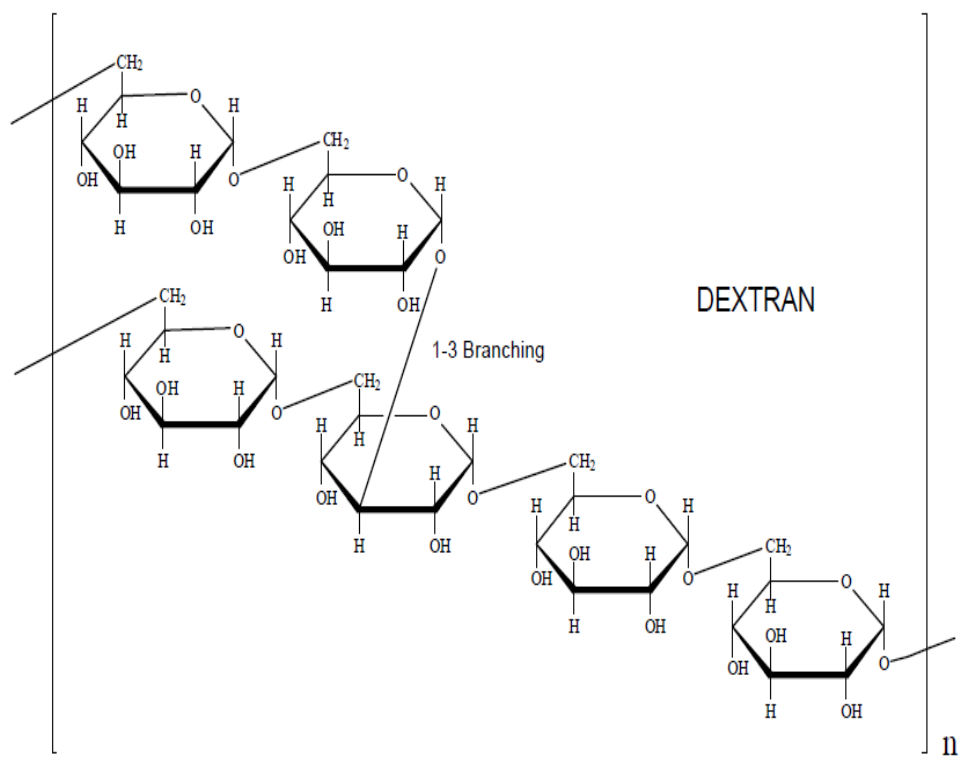

(b)

Figure 2-16: (a) Structure of the reactive blue dye; and (b) Structure of the dextran unit. 
Blue dextran was purchased from Sigma Aldrich. Stock solution of the blue dextran was prepared with DI water. In Figure 2-17, the absorbance measurements for various bulk concentrations $(0.1,0.3$ and $0.6 \mu \mathrm{M})$ were measured with a conventional spectrophotometer. The pure DI water solution was collected as the reference baseline. The same absorbance peak at $620 \mathrm{~nm}$ was observed. With the measured absorbance results, the molar absorptivity of blue dextran was calibrated $\left(\epsilon(560 \mathrm{~nm})=1.2 \times 10^{3} \mathrm{M}^{-1} \mathrm{~cm}^{-1}\right.$ and $\left.\epsilon(570 \mathrm{~nm})=1.4 \times 10^{3} \mathrm{M}^{-1} \mathrm{~cm}^{-1}\right)$.

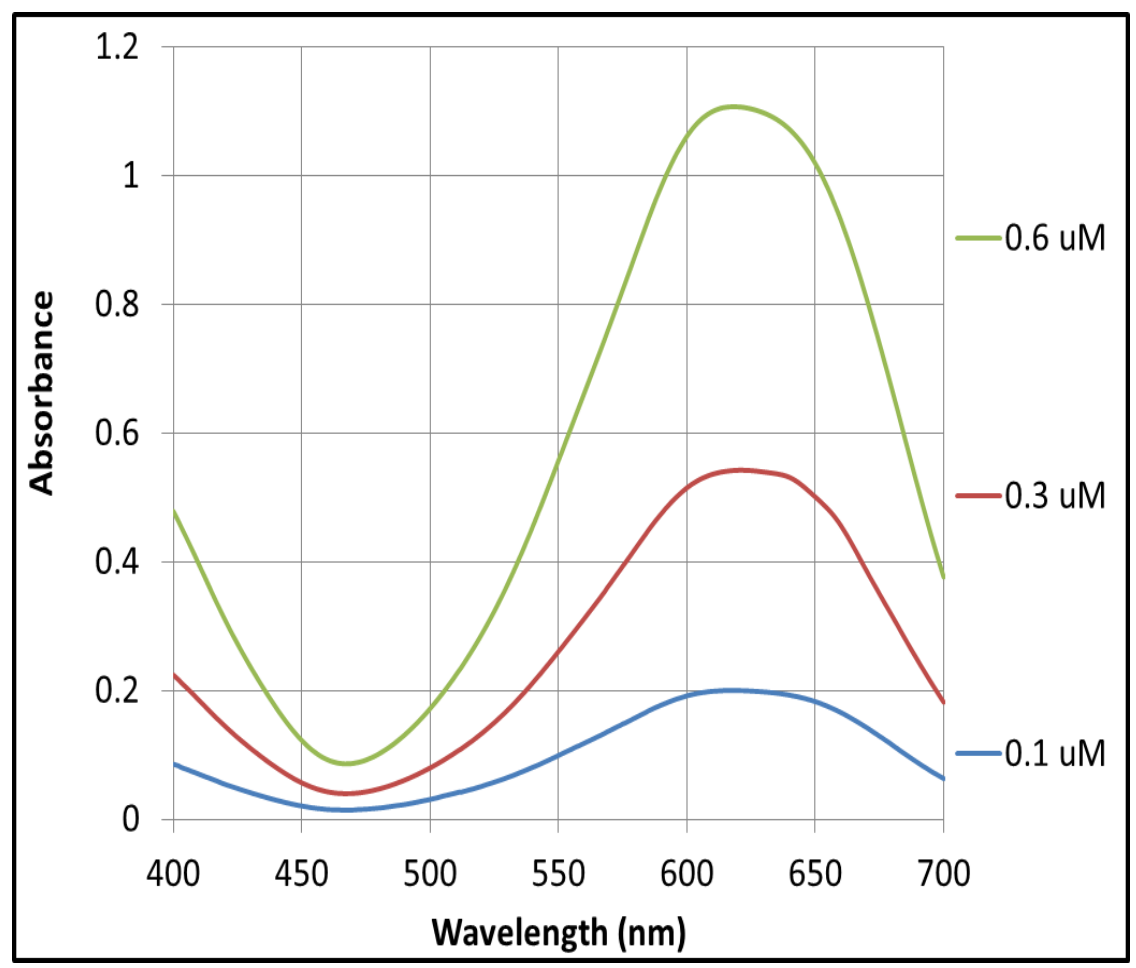

Figure 2-17: Absorbance curves of various bulk concentrations of blue dextran in DI water measured with the transmission probing structure. 
The same bulk concentrations of blue dextran were tested with an EA-SMIOW. The absorbance results are shown in Figure 2-18. For the case that no formation of a molecular thin film on the surface of the device, the absorbance is exclusively from dissolved species, and the sensitivity of an EA-SM-IOW device can be expressed in Equation 2-6 [137], where $\epsilon_{\text {bulk }}$ is the molar absorptivity of blue dextran and $c_{\text {bulk }}$ is the bulk concentration.

$$
S=\frac{A b s_{I O W}}{A b s_{T} \frac{\lambda}{4 \pi\left(N_{e f f^{2}}^{2} n_{C}^{2}\right)^{\frac{1}{2}}}}=\frac{A b s_{I O W}}{\epsilon_{\text {bulk }} c_{b u l k} \frac{\lambda}{4 \pi\left(N_{e f f^{2}}^{2} n_{C}^{2}\right)^{\frac{1}{2}}}}
$$

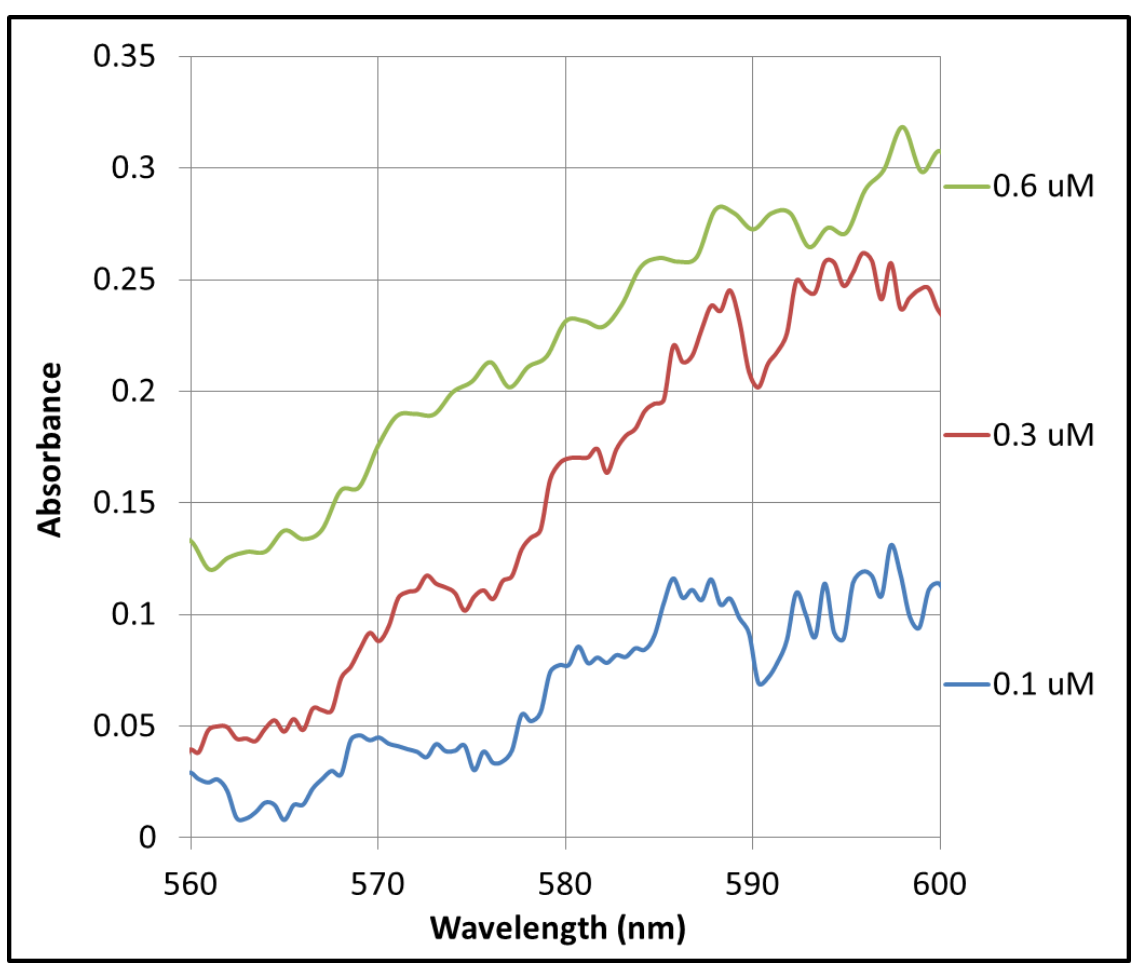

Figure 2-18: Absorbance curves for various bulk concentration of blue dextran in DI water measured with the EA-SM-IOW probing structure. 
The absorbance measured by the device, $A b s_{I O W}$ against $\epsilon_{b u l k}$ is plotted in Figure 2-19 for wavelength $560 \mathrm{~nm}$ and $570 \mathrm{~nm}$. A linear fit was used, and the fitted slope equaled to $\frac{S \epsilon_{\text {bulk }} \lambda}{4 \pi\left(N_{e f f}^{2}-n_{c}^{2}\right)^{\frac{1}{2}}}$. Since the other parameters in Equation 2-6 were constants, the sensitivity factor was calculated.

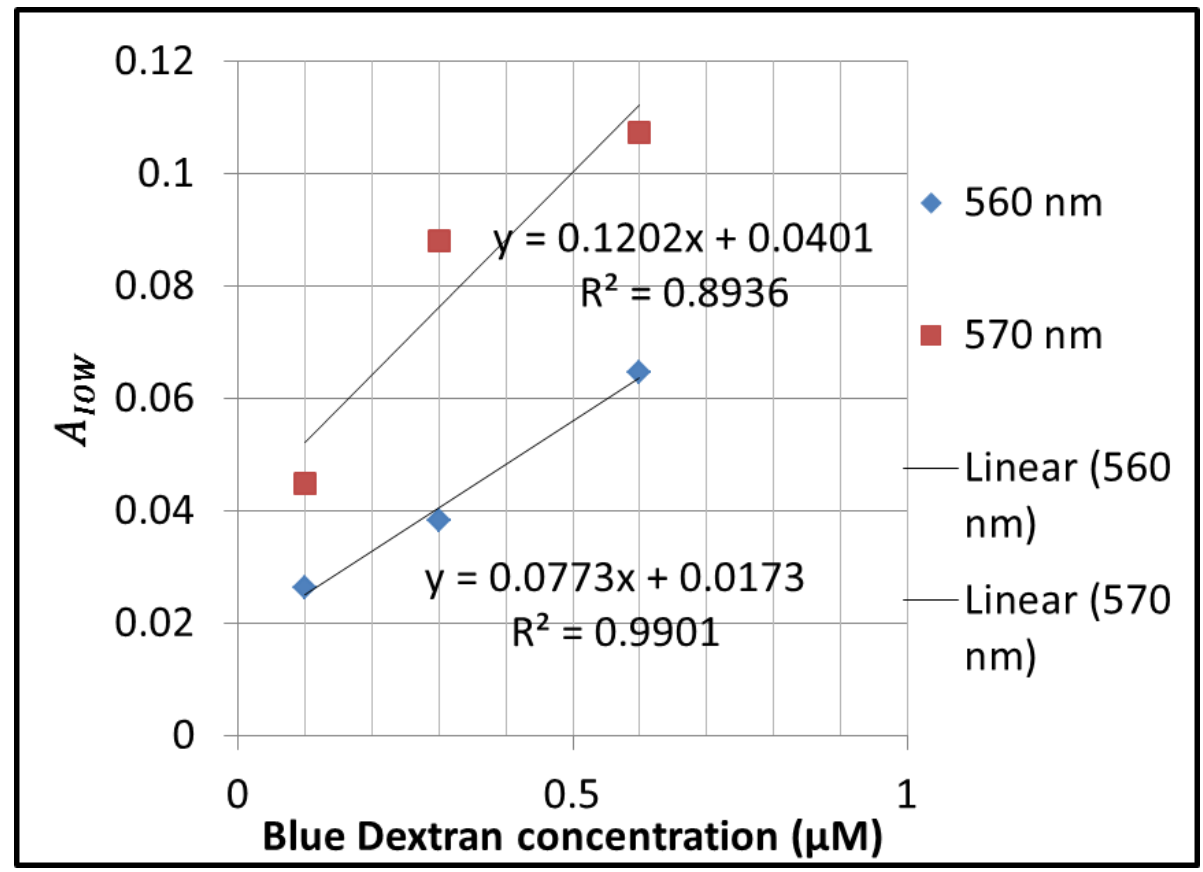

Figure 2-19: Absorbance plot against bulk concentration of blue dextran measured by EA-SM-IOW at $560 \mathrm{~nm}$ and $570 \mathrm{~nm}$. Linear fitting is used. The slope of the fitted results is used to calculate the sensitivity factor.

These measurements provide a sensitivity factor of 13029 at $560 \mathrm{~nm}$ and 17007 at $570 \mathrm{~nm}$, respectively. These values agree well with the theoretical 
calculation based on the optical constants and thickness of each layer, as discussed in section 2.5.4.

\section{Propagation loss}

The extinction coefficient measurements of the thick ITO film after the inert annealing from Section 2.4.2, can be used to calculate the propagation loss with Equation 2-7, where $t_{\text {ITO }}=13 \mathrm{~nm}$ for the ultra-thin ITO layer, $\lambda=543 \mathrm{~nm}$ (used for the loss measurement), $S=14313$ calculated at $543 \mathrm{~nm}$, and $\kappa_{\text {ITO }}=1.7 \times 10^{-4}$. And the results from this calculation and the experimental measurement for the optimized EA-SM-IOW in Section 2.4.3 are compared.

$$
\begin{aligned}
& A b s_{I O W, I T O}=S A b s_{T, I T O}=S t_{I T O} \frac{4 \pi \kappa_{I T O}}{\lambda \ln (10)}=0.32 / \mathrm{cm} \\
& \operatorname{LoSS}(I T O)=10 A b S_{I O W, I T O}=3.2 \mathrm{~dB} / \mathrm{cm}
\end{aligned}
$$

Before the deposition of the ultra-thin ITO film, the propagation loss of a SMIOW slide (aluminum oxide and the silicon dioxide layers) was approximately 2 $\mathrm{dB} / \mathrm{cm}$. Considering the loss from the SM-IOW slide, this calculated propagation loss matched reasonably well with the measured total loss of $6.0 \mathrm{~dB} / \mathrm{cm}$ for the whole sack after the active annealing process used to optimize the ultra-thin ITO film.

\section{Conclusions}

The design, fabrication, and performance of the EA-SM-IOW were described. The high requirements for the transparent conductive layer were fulfilled. The EASM-IOW device was featured with an extremely low optical propagation loss and a 
low resistivity for electrochemical potential modulations. The high sensitivity of the device was calculated theoretically by using the optical constants and thicknesses of each layer in the multi-layer waveguide structure. The sensitivity factor was also demonstrated experimentally with absorbance measurements. An agreement with the theoretical and experimental results was shown. 


\title{
CHAPTER 3
}

\section{SPECTROSCOPIC FEATURES OF ULTRA-THIN ITO FILM UNDER POTENTIAL MODULATION}

\author{
Introduction
}

Transparent conductive electrodes (TCE) have been used in many technological and scientific application areas [138-145]. Various materials for TCEs, such as metal thin films, different formats of carbon, organic polymers, and semiconductors have been utilized. Metal oxides (ITO, $\mathrm{ZnO}[146,147], \mathrm{WO}_{3}$, CdO, FTO, $\mathrm{TiO}_{2}$ [148], $\mathrm{SnO}_{2}$ ) are popular for TCEs because of their high transparency in the visible range and the low resistivity [149-152].

Among the metal oxide materials, n-type semiconductor ITO has shown superior qualities [153]. These films have been applied in switches for MEMS devices [154], sensors $[155,156]$, large area display panels [157], solar cells [158160] and light emitting diodes [161-163]. The static electrical and optical properties of ITO films at varied thicknesses have been studied thoroughly with various deposition techniques and annealing conditions [164]. The films can have a wide band gap between $2.7 \mathrm{eV}$ [165] to $4.6 \mathrm{eV}$ [166]. The electrical properties of the thick ITO films (approximately $\mu \mathrm{m}$ ) under potential modulations in acid, basic or high 
ionic strength solutions have been studied [167-173]. Research has also been done on the surface characteristics (x-ray photoelectron spectroscopy) and the bulk features (UV photoelectron spectroscopy) of ITO films [174]. Very few studies have been carried out to understand the kinetics of the electrical and the optical properties of an ultra-thin ITO film (smaller than $50 \mathrm{~nm}$ ) under potential modulations in a liquid phase. In this chapter, the spectroscopic features of ultrathin ITO films under various potential modulation techniques are described and compared. For the applications of an EA-SM-IOW in spectroelectrochemistry, the importance of these measurements is discussed in later chapters.

\section{Spectroelectrochemical Flow Cell}

An electro-active single-mode integrated optical waveguide (EA-SM-IOW) with an optimized ultra-thin ITO layer was put in buffer solution $\left(\mathrm{Na}_{2} \mathrm{PO}_{4}, 5 \mathrm{mM}\right.$ concentration and approximately pH 7) for at least 24 hours. After this stabilization process, the device was dried with $\mathrm{N}_{2}$ gas. The surface of the device was examined and cleaned with acetone. A piece of carbon tape was used to fix a platinum wire on the ITO film to connect the EA-SM-IOW to the potentiostat (CHI 660D). A layer of silver paste was circled around the two gratings to provide a uniform electric potential for the whole ITO surface. The light path between the two gratings was not disturbed. After the silver paste was dry, an insulating layer of epoxy was coated on top of the silver paste to avoid any chemical contact between the silver paste and the buffer solution. For a proper electrical connection package, the resistance between the epoxy layer and the platinum wire should be enormous, and the 
resistance between the Pt wire and ITO film should be close to the sheet resistance. The architecture of the electrochemical flow cell is shown in Figure 3-1. A pseudo $\mathrm{Ag} / \mathrm{AgCl}$ reference electrode and a Pt counter electrode were mounted on the cover of the flow cell. A rubber gasket was used to seal the flow cell and provide a uniform pressure to the device when the flow cell cover was screwed down. The volume of the flow cell was approximately $2 \mathrm{ml}$.

Cyclic voltammetry (CV) potential scans from $+0.8 \mathrm{~V}$ to $-0.4 \mathrm{~V}$ with scan speeds of $0.02 \mathrm{~V} / \mathrm{s}, 0.1 \mathrm{~V} / \mathrm{s}$ and $0.2 \mathrm{~V} / \mathrm{s}$ were used to stabilize the ITO film. More than 10 cycles of potential scans were applied until the measured electrical current signal was stabilized.

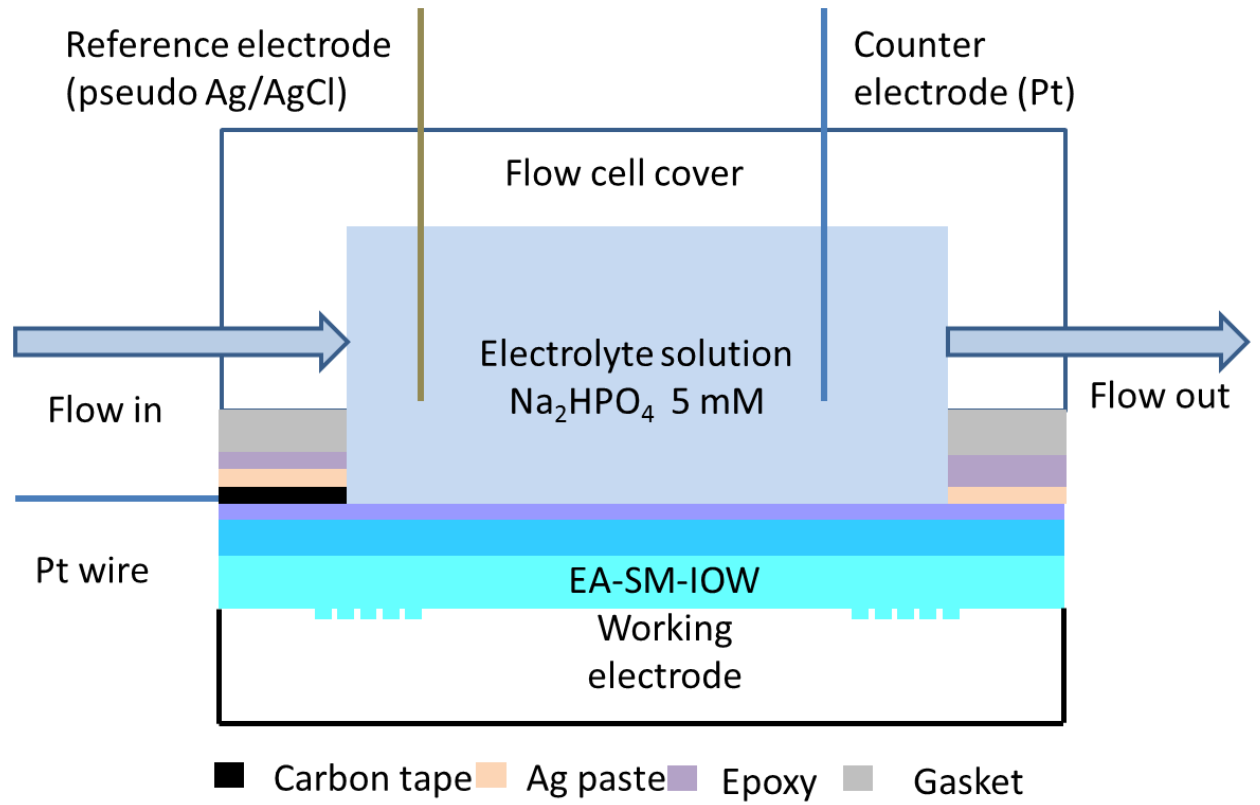


Figure 3-1: Cross section of the electrical contacts for an EA-SM-IOW in the spectroelectrochemical flow cell. Different materials used for the electrical connection package are demonstrated with different colors.

\title{
Ultra-thin ITO under Potential Modulations
}

\author{
Cyclic voltammetry
}

The same stationary optical coupling set-up, as shown in Figure 2-10, was used. A tungsten-halogen white light lamp was used as the light source. An ICCD camera connected by a monochromator was used to collect the out-coupled spectra. Figure 3-2 shows the experimental set-up. A CV potential scan with a speed of 0.02 $\mathrm{V} / \mathrm{s}$ from $+0.8 \mathrm{~V}$ to $-0.4 \mathrm{~V}$ is plotted against the time in Figure 3-2 (top inset). A computer was connected to the potentiostat and the ICCD camera to control the potential modulation and the data collection, respectively. The setting of the ICCD camera was based on the potential scan speed. The internal trigger of was set at 2 $\mathrm{Hz}$, and exposure time was set at $200 \mathrm{~ms}$ for each frame. The electrical current signal and the optical spectra were collected simultaneously. 


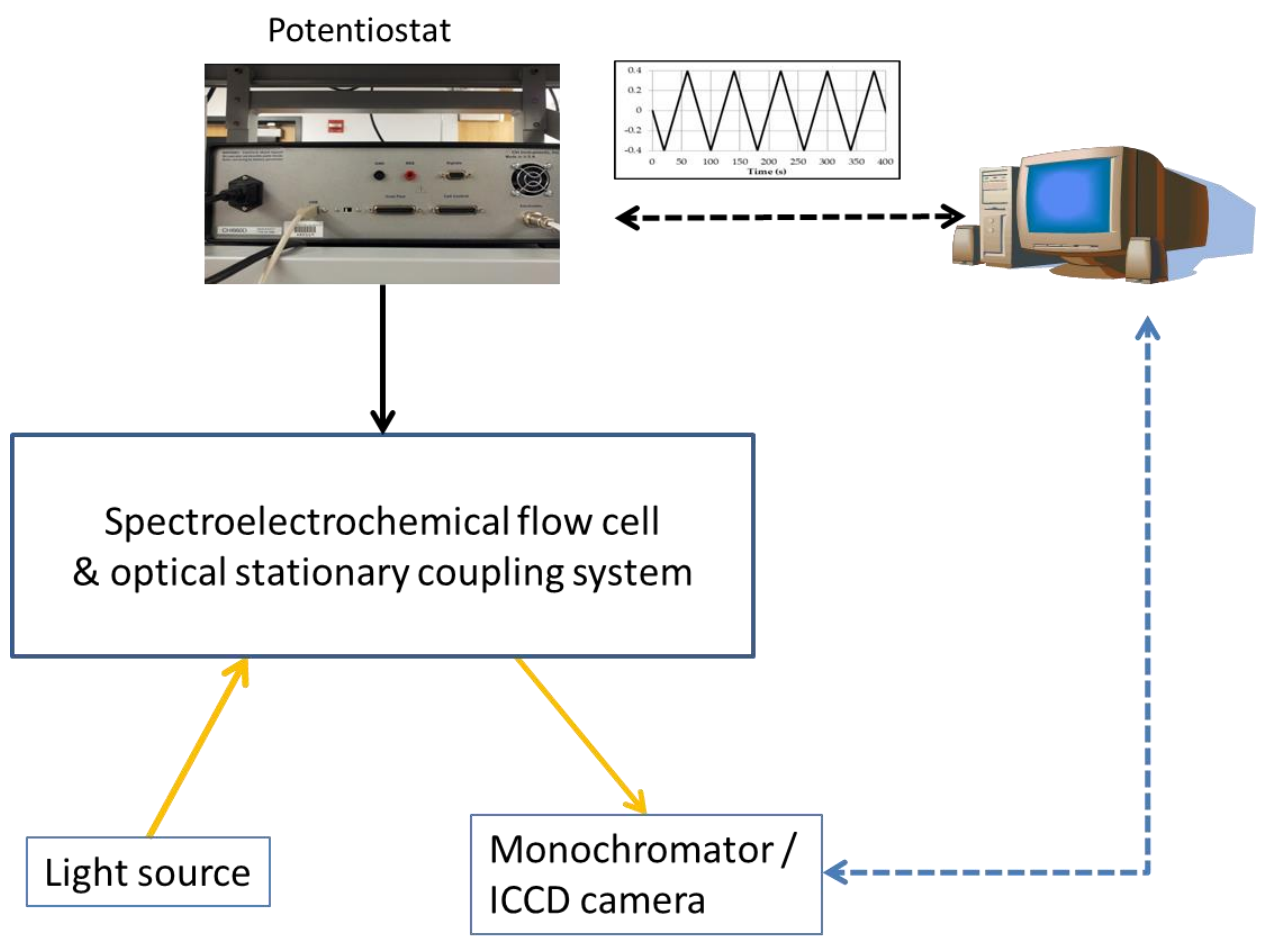

Figure 3-2: Experimental set-up with the stationary coupling set-up for the CV potential scan. A CV potential modulation with $0.02 \mathrm{~V} / \mathrm{s}$ scan speed is shown in the inset on top.

Since the potential scan had a slow speed, the optical spectra and the applied potential could be assigned point by point. The out-coupled spectra profiles at different potential points during the $\mathrm{CV}$ scan are shown in Figure 3-3. The differences between the profiles at selected potential points are clear. At $0.4 \mathrm{~V}$, the spectrum has the broadest and highest profile. At $-0.4 \mathrm{~V}$, the spectrum has the narrowest and lowest profile. 


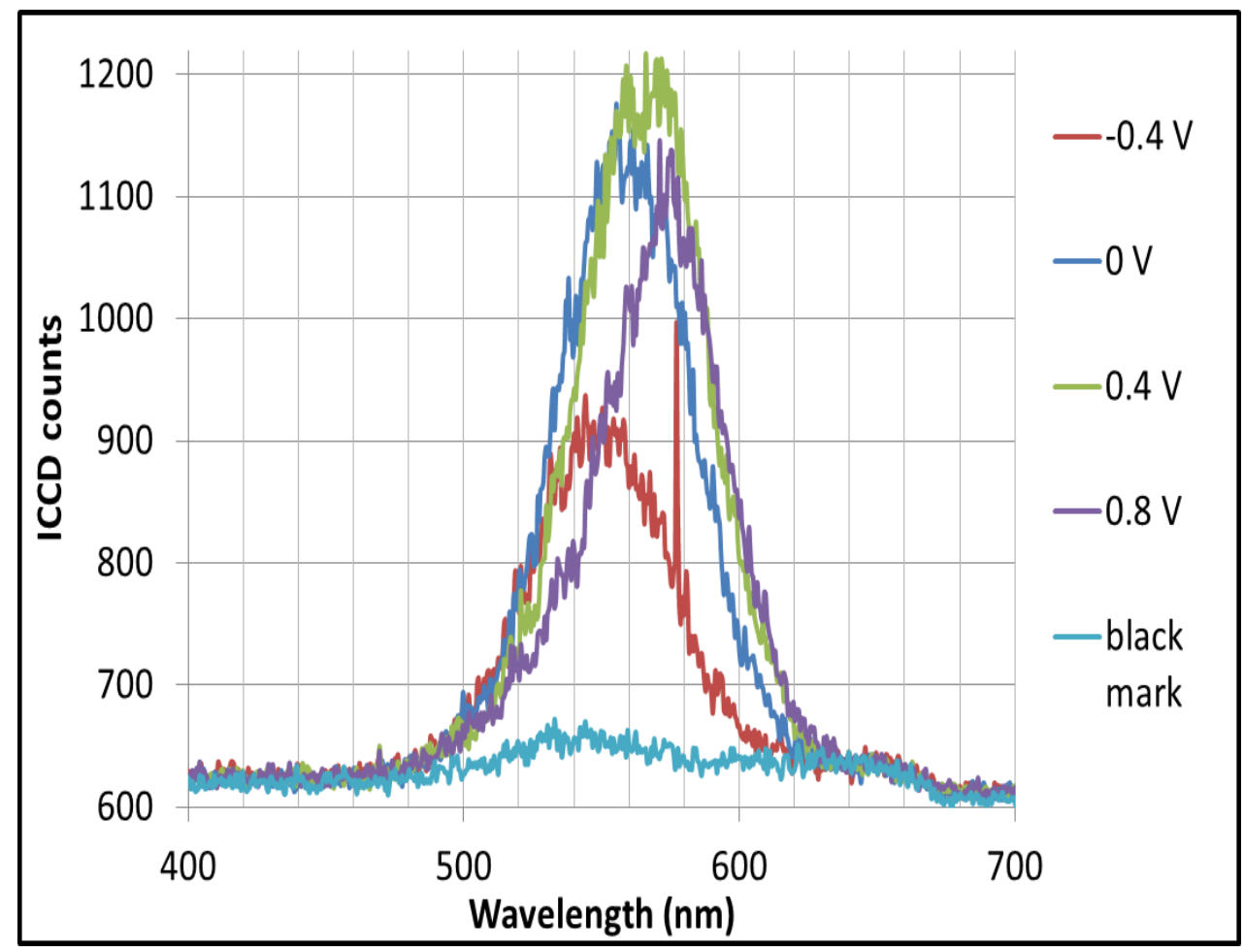

Figure 3-3: Out-coupled spectra at different potential points during the CV scan.

In the measurements above, the coupling angle was fixed and a broadband spectrum was used. The same CV potential scan was tested repeatedly with several monochromatic laser beams, and the flow cell was mounted on a rotation stage to adjust the incident angle for each laser line for the highest coupling efficiency (coupling angle). The experimental st-up is shown in Figure 3-4. A polarizer was used to select the TE polarized incident light. The out-coupled light was collected with a short focal length lens and coupled into a fiber which was connected to the monochromator and the ICCD camera. One trace of the out-coupled light intensity under the CV potential scan is illustrated in the inset of Figure 3-4. 


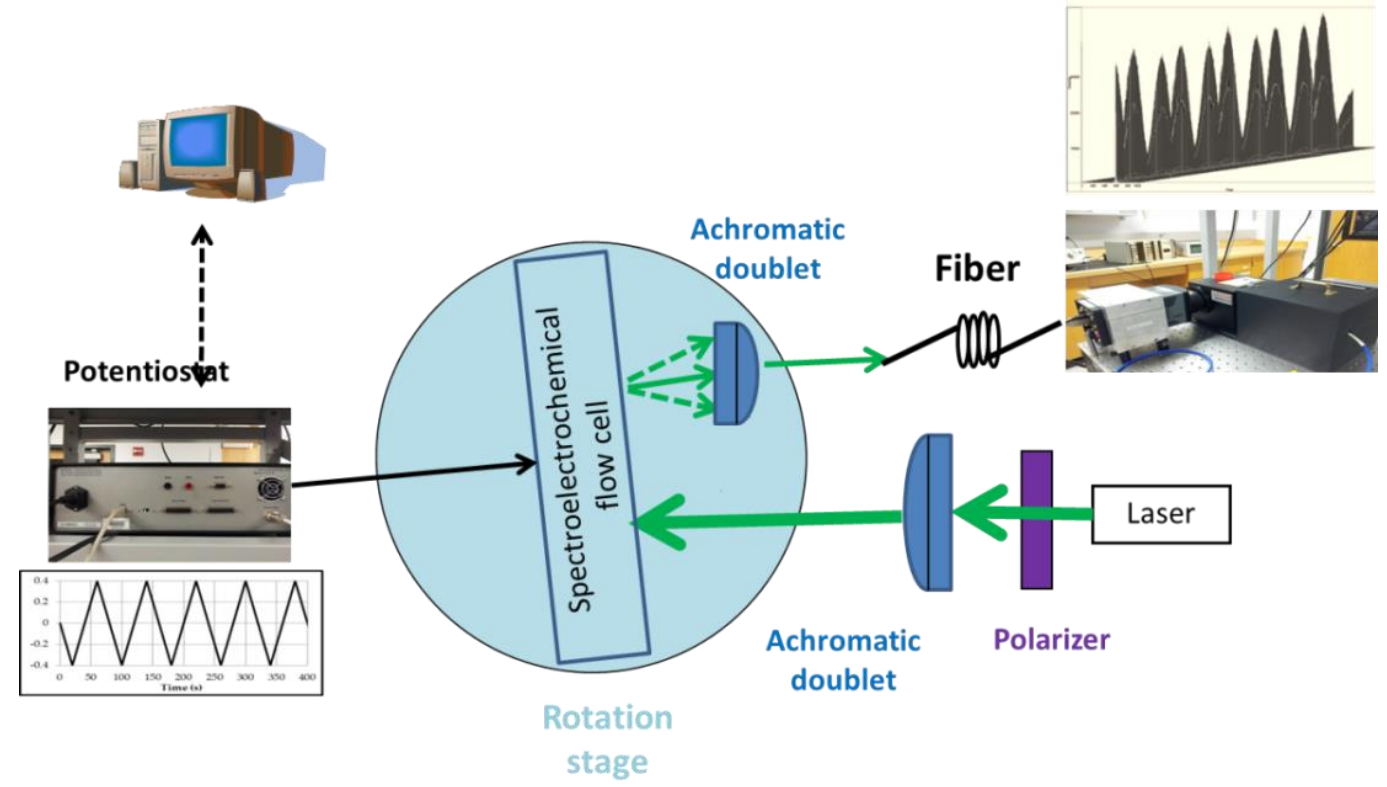

Figure 3-4: Rotation coupling set-up for the cyclic voltammetry potential scan with a laser beam as the light source. The coupling angle for each laser beam is achieved by rotating the stage. One trace of the out-coupled intensity against time collected by the ICCD camera is shown in the top inset.

Normalized transmittances $\left(0<t_{N}<1\right)$ were calculated with 3-1 to show the spectroscopic features of the ultra-thin ITO film under the CV scan.

$$
t_{N}(E, \lambda)=\frac{I_{E}-I_{\min }}{I_{\max }-I_{\min }}
$$

For a specific wavelength $\lambda, I_{E}$ is the out-coupled intensity at potential $E . I_{\min }$ and $I_{\max }$ are the minimum and maximum of out-coupled intensities inside the scanning region of $+0.8 \mathrm{~V}$ to $-0.4 \mathrm{~V}$. The normalized transmittance curves $t_{N}$ are plotted against time for a 5-cycle CV scan, as shown in Figure 3-5 (a) for different laser lines. The curves are shifted evenly by one unit for a better visualization. The 
$\mathrm{CV}$ potential scan is also plotted against time as shown in the black trace. The repeatability of the optical response from each wavelength under a 5-cycle CV scan is clear. The folded 5-cycle of normalized transmittance against potential is shown in Figure 3-5 (b) for each laser beam. For wavelengths longer than $600 \mathrm{~nm}$, the transmittance follows the potential scan, i.e. they increase and decrease together. At $502 \mathrm{~nm}$, the transmittance and the potential scan change in opposite directions. The normalized transmittance curve at $543 \mathrm{~nm}$ shows both behaviors depending on the value of the potential.

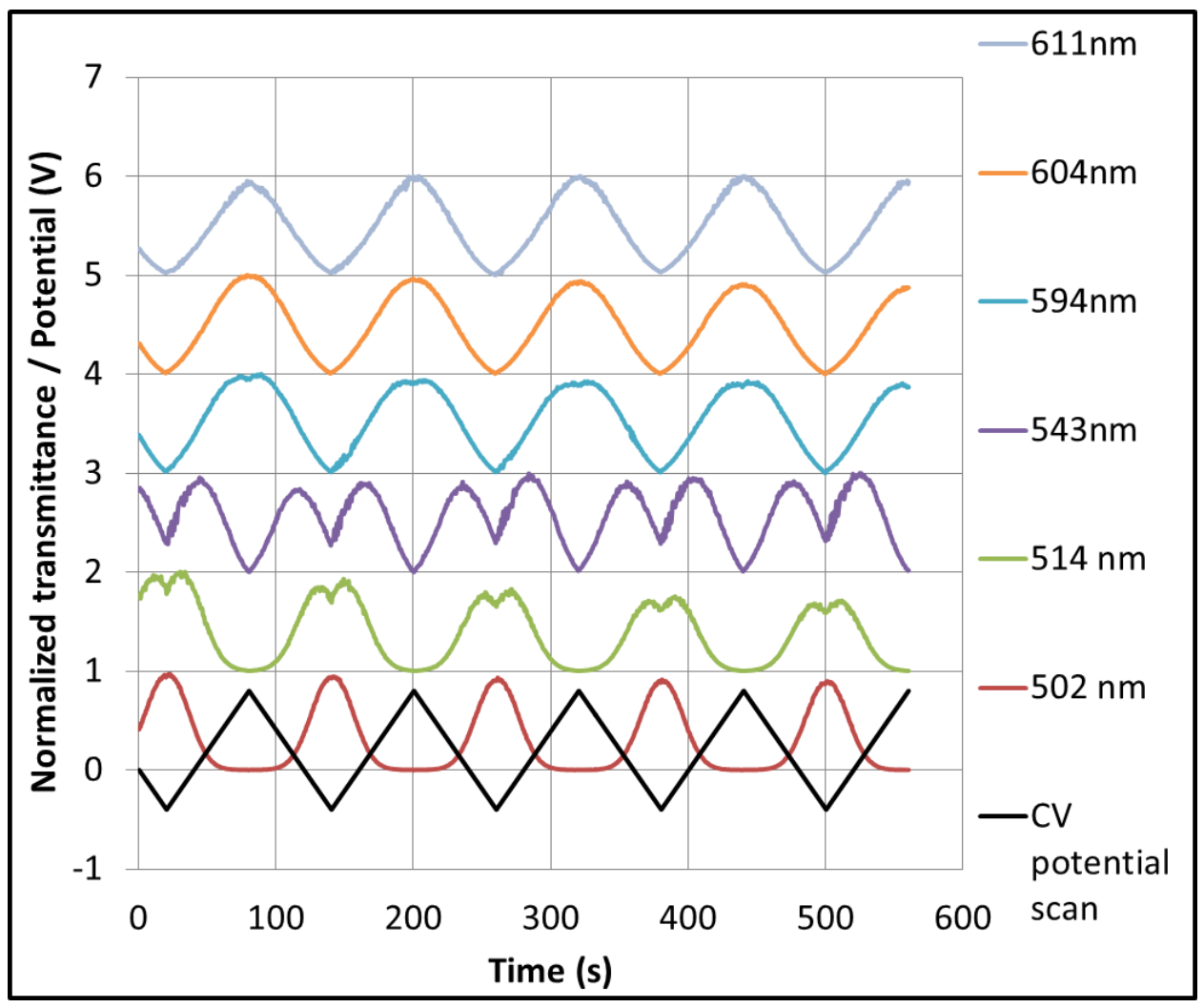

(a) 


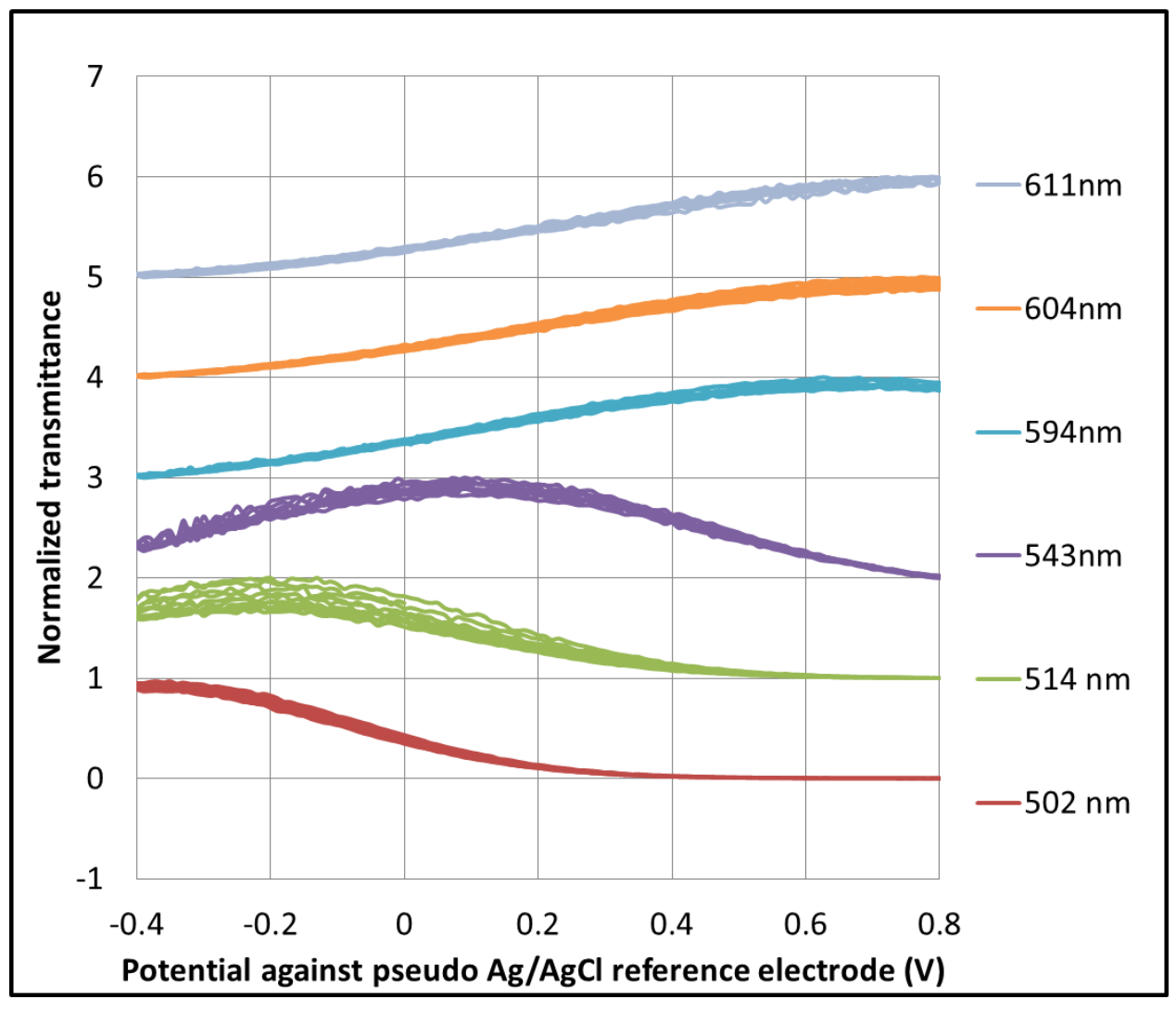

(b)

Figure 3-5: (a) Normalized transmittance for different laser beams under the same $\mathrm{CV}$ potential scan. Potential modulation is plotted in the same graph against time as in black curve. Each curve is shifted by one unit. (b) Folded 5-cycles of normalized transmittance against the applied potential is plotted to show the high repeatability of the spectroscopic features.

The same CV potential scan was also used to examine the ultra-thin ITO films with different polarization for the incident light (TE and TM) and different thicknesses (8 $\mathrm{nm}$ and $13 \mathrm{~nm}$ ) (Appendix 4). The normalized transmittance profiles are similar for these different conditions. 
The electrical current response under the CV scan was collected from the experiments using individual laser lines, as shown in Figure 3-6. The electrical current curves show no obvious sign of current peaks associated with redox reactions. The previous results of out-coupled intensity with a wavelengthdependence under potential modulation suggest that those spectroscopic features are related to the ultra-thin ITO film.

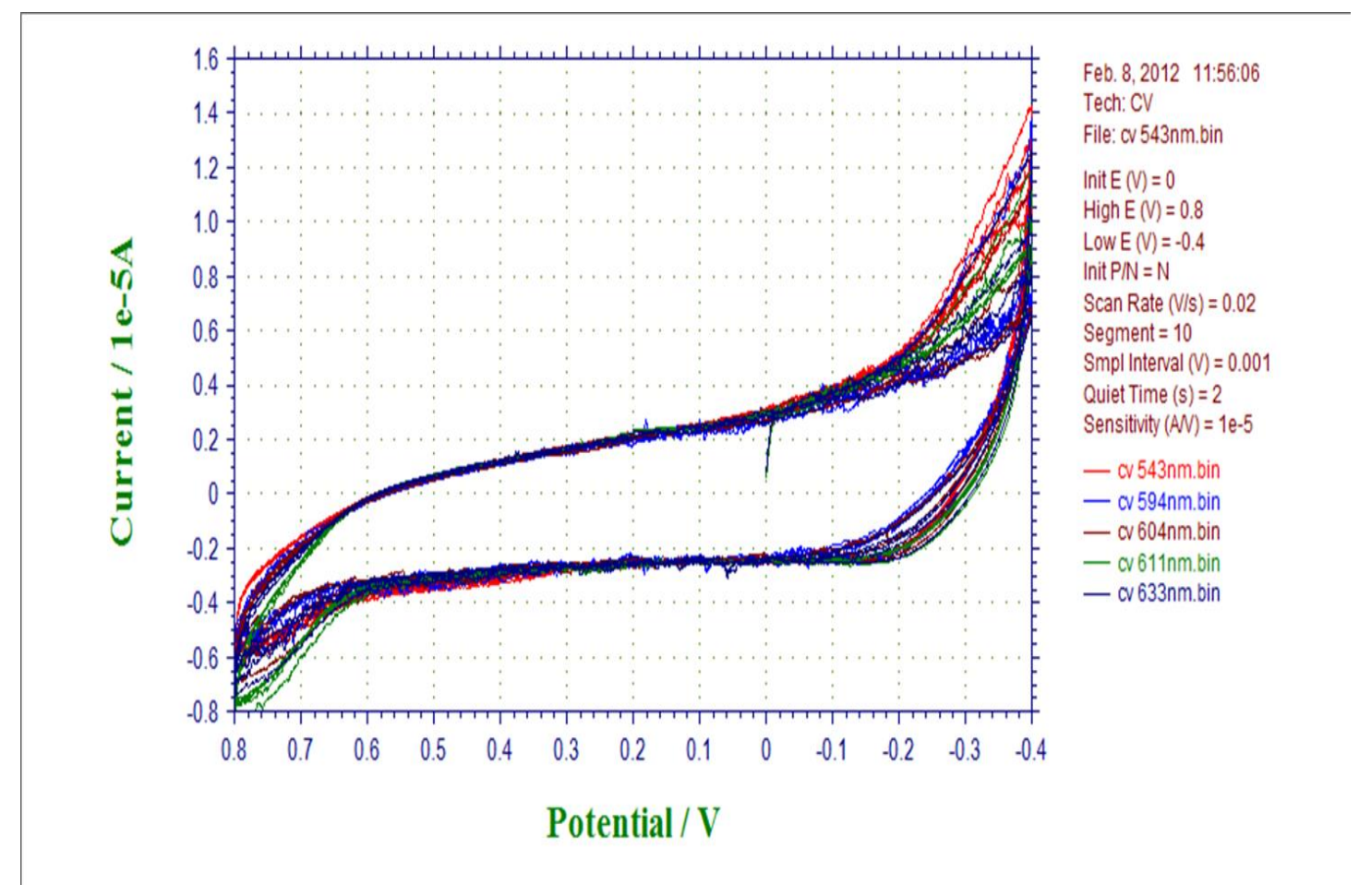

Figure 3-6: Electrical current curves under the same the CV potential scan when various laser beams are used as probing light sources. For the same device, the same CV scan results similar current curve. Anodic current is set as the positive current. 


\section{Fixed potential steps}

The potential modulation can modified the real and imaginary components of the refractive index of an ultra-thin ITO film. The phenomenon that the real part of the refractive index of an ITO film can be modified by applying a potential modulation has been studied in the context of biosensor applications $[175,176]$.

An experiment was designed to identify the dominant effect, a modification of the real or the imaginary component of the refractive index. A change in the real part of the refractive index of an ultra-thin ITO film would result in a change of the effective refractive index of the multi-layer structure, as described in section 2.1.2. A change in the effective refractive index would cause a shifted coupling angle. The coupling angle could result in the portion of the incident light coupled into the single-mode waveguide oscillating. A change in the imaginary of an ultra-thin ITO film could result in a modified attenuation. During the potential modulation, an oscillation of the attenuation of the ultra-thin ITO film could result in the optical loss and out-coupled intensity oscillating.

To investigate this phenomenon, the rotation coupling set-up with the rotation stage was used, the same as shown in Figure 3-4. If the change in the real part of the refractive index is the dominant factor, a strong difference in the coupling angle would be observed between different potential values. If the imaginary part of the refractive index is the dominant factor, the angle of the maximum coupling at each potential value would be similar, but the out-coupled light intensity would show a strong dependency on the applied potential. 
The measurement was carried out first at the open circuit potential to select the best coupling point of the grating edge. Then the position of the flow cell on the rotation stage was fixed. By rotating the stage, the incident angle was scanned. The out-coupled light was collected by an achromatic lens and measured by a power meter. The constant potential step had $1000 \mathrm{~s}$ duration. The electrical current signal was monitored. Only when the current signal was stabilized, the out-coupled intensity was recorded for each incident angle.

Figure 3-7 shows the result of the out-coupled intensity profile for $514 \mathrm{~nm}$ (a) and $594 \mathrm{~nm}$ (b) laser beams. For both probing wavelengths, the coupling angles for different potential steps are not dramatically shifted from each other; however the light intensity (thus attenuation) changes dramatically.

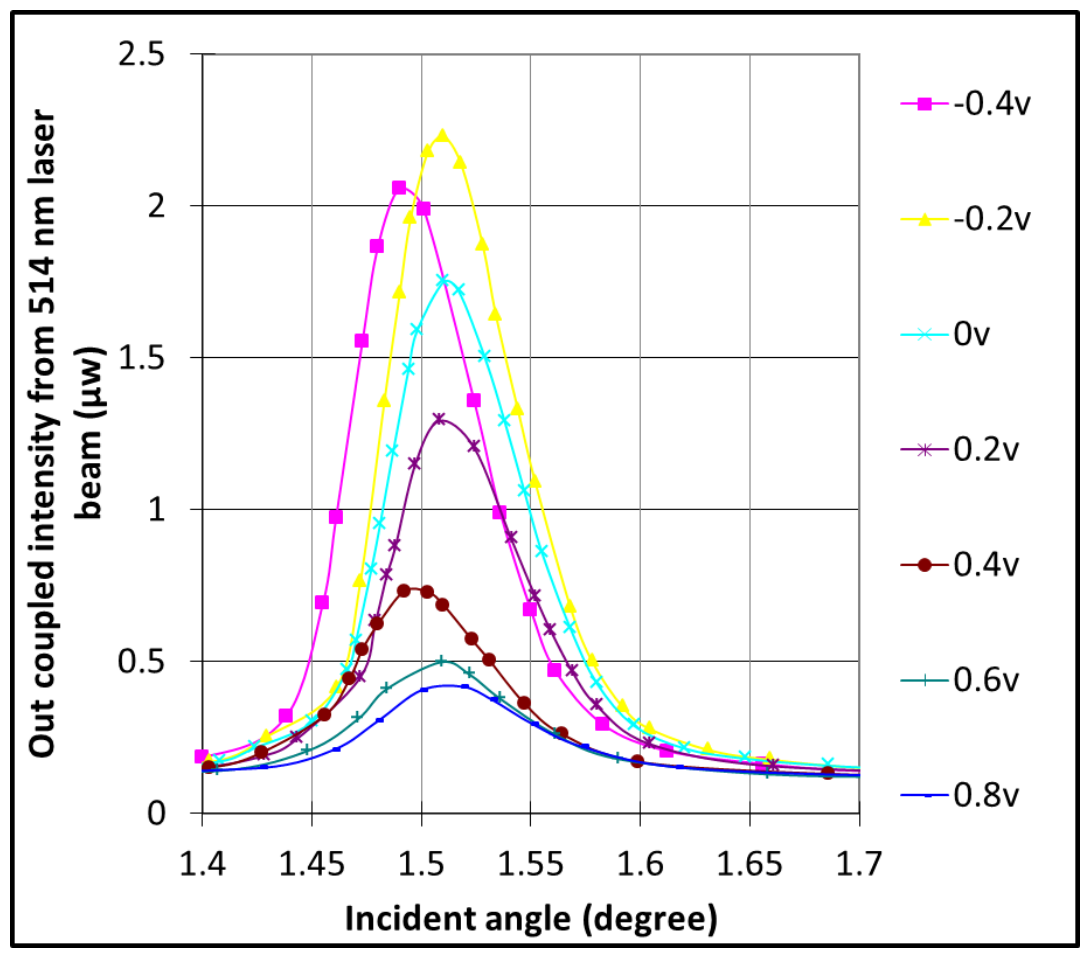

(a) 


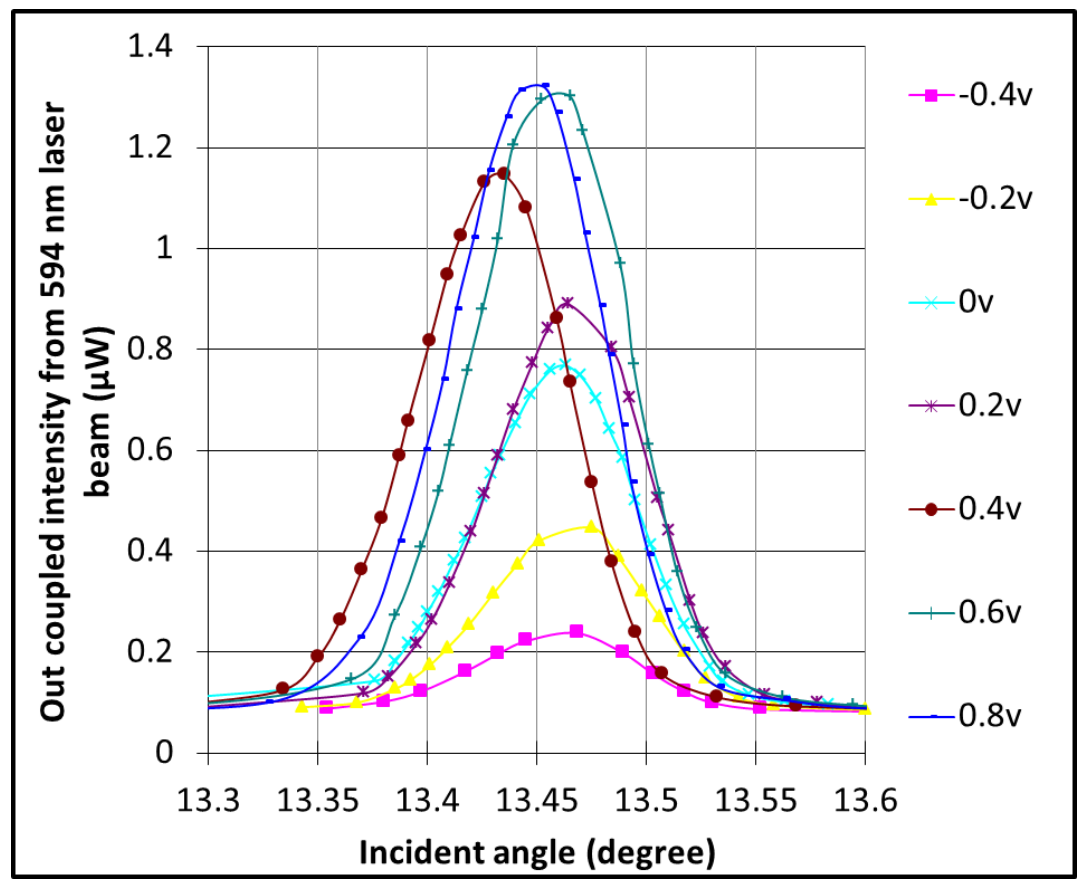

(b)

Figure 3-7: Out-coupled intensity against incident angle profiles by using (a) 514 $\mathrm{nm}$ and (b) $594 \mathrm{~nm}$ laser beams as probing light sources at different constant potential steps.

For $514 \mathrm{~nm}$, the lowest and highest profiles are at $+0.8 \mathrm{~V}$ and $-0.2 \mathrm{~V}$ potential steps respectively. The maximum intensity $(0.43 \mu \mathrm{W})$ at $+0.8 \mathrm{~V}$ is approximately 5 times lower than the maximum at $-0.2 \mathrm{~V}(2.23 \mu \mathrm{W})$. For $594 \mathrm{~nm}$, the lowest and highest intensity profiles are at $-0.4 \mathrm{~V}$ and $+0.8 \mathrm{~V}$. The maximum $(1.32 \mu \mathrm{W})$ at $+0.8 \mathrm{~V}$ is more than 5 times higher than that from $-0.4 \mathrm{~V}(0.24 \mu \mathrm{W})$. This is another demonstration of the spectroscopic features of the ultra-thin ITO film under an applied potential. Results from other laser beams are shown in Appendix 5. 
The effective refractive indices were calculated from the measured incident angle with the highest out-coupled intensity for a particular applied potential with Equation 2-1. The results are summarized in Figure 3-8 for each laser line. The effective refractive index of the EA-SM-IOW stayed almost as constant, when different potential steps were applied. This result could be understood from theoretical considerations that the ITO layer was ultra-thin (approximately $13 \mathrm{~nm}$ ) and therefore a change in the real part of the refractive index had little impact on the effective refractive index of the overall structure, as shown in section 2.5.3.

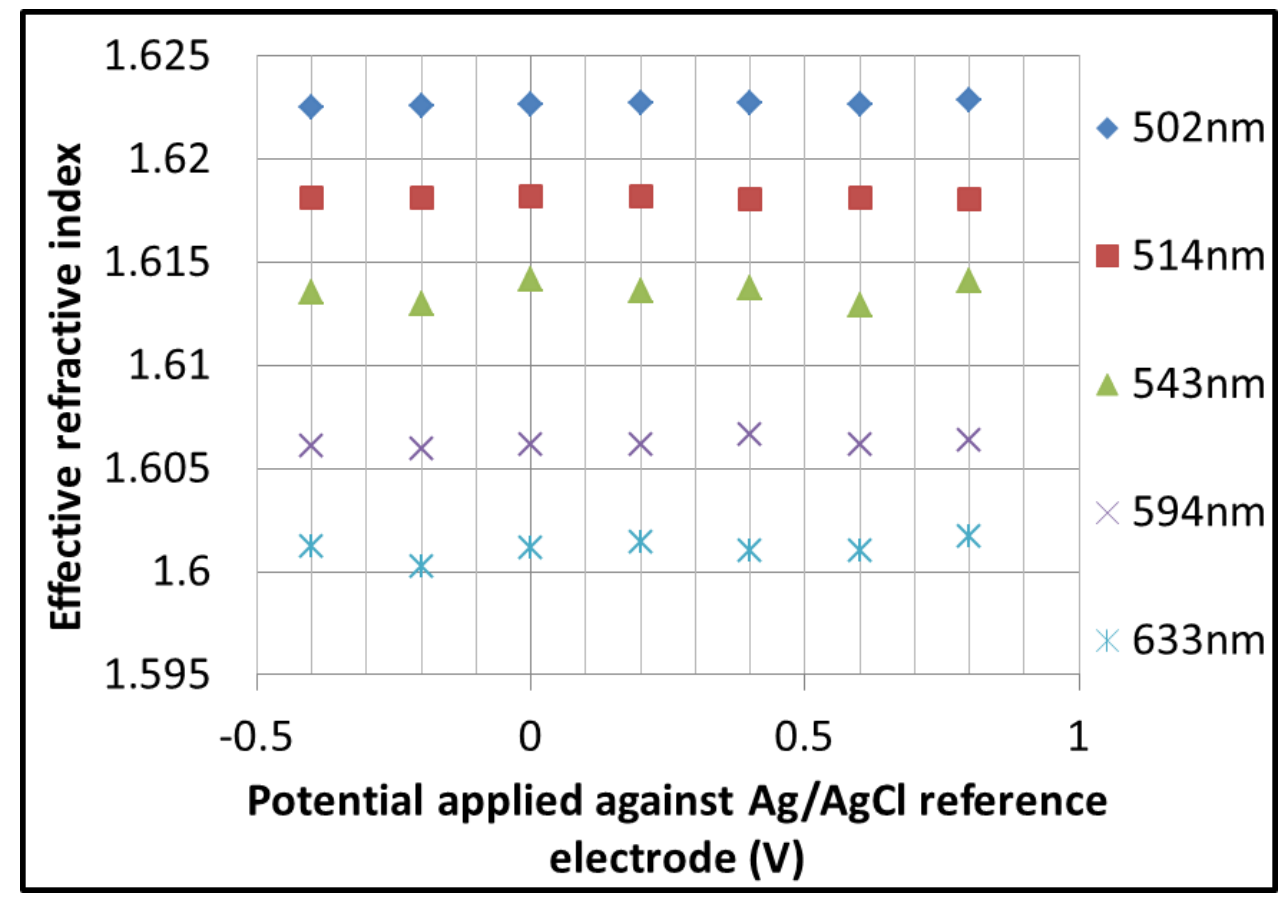

Figure 3-8: Effective refractive index for an EA-SM-IOW electrode in neutral buffer solution at various constant potential steps. 
Normalized transmittance profiles were also calculated and compared to the data from the CV scan results, as shown in Figure 3-9 (a) for $514 \mathrm{~nm}$ and (b) for 594 $\mathrm{nm}$ respectively. The angle of the maximum coupling efficiency at the open circuit potential was chosen for constant potential step measurements to match the condition of the CV scan experiments. An agreement between the two types of measurements was observed.

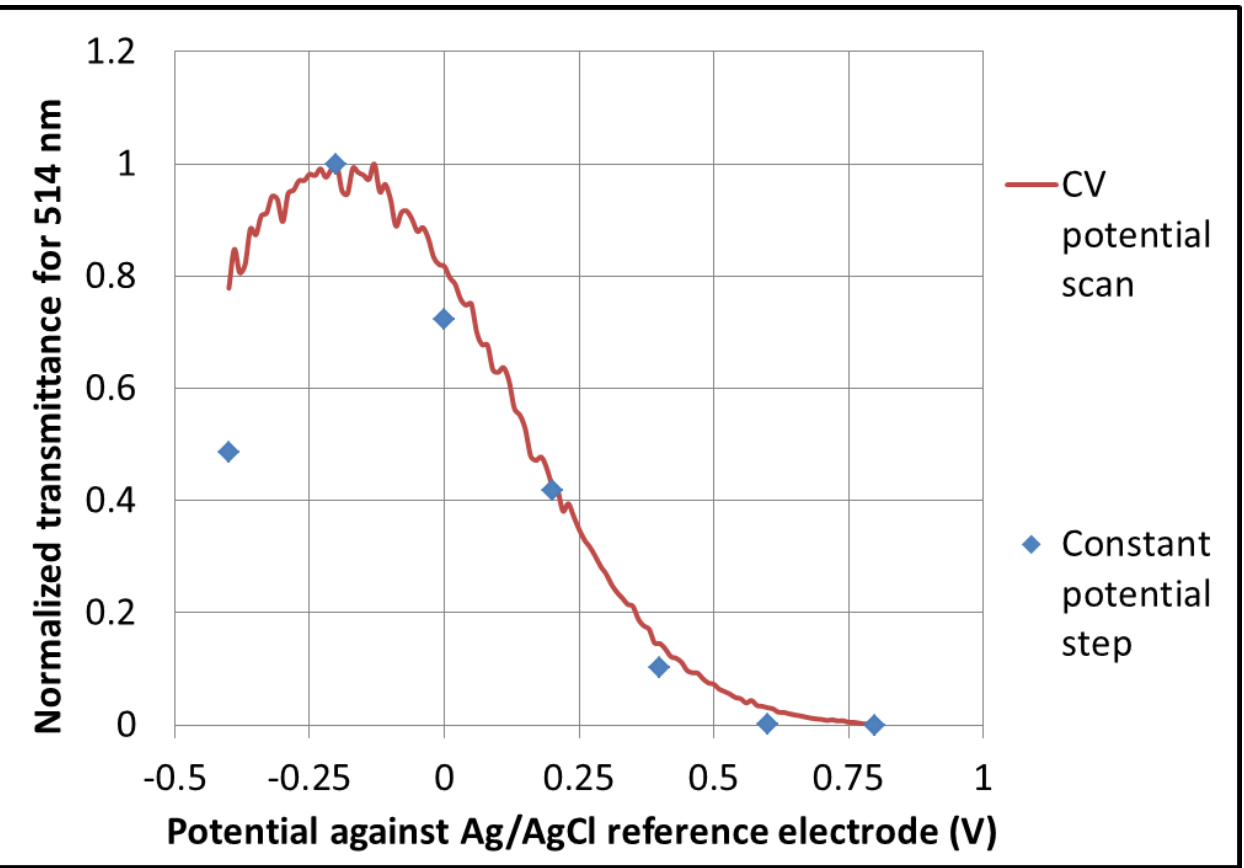

(a) 


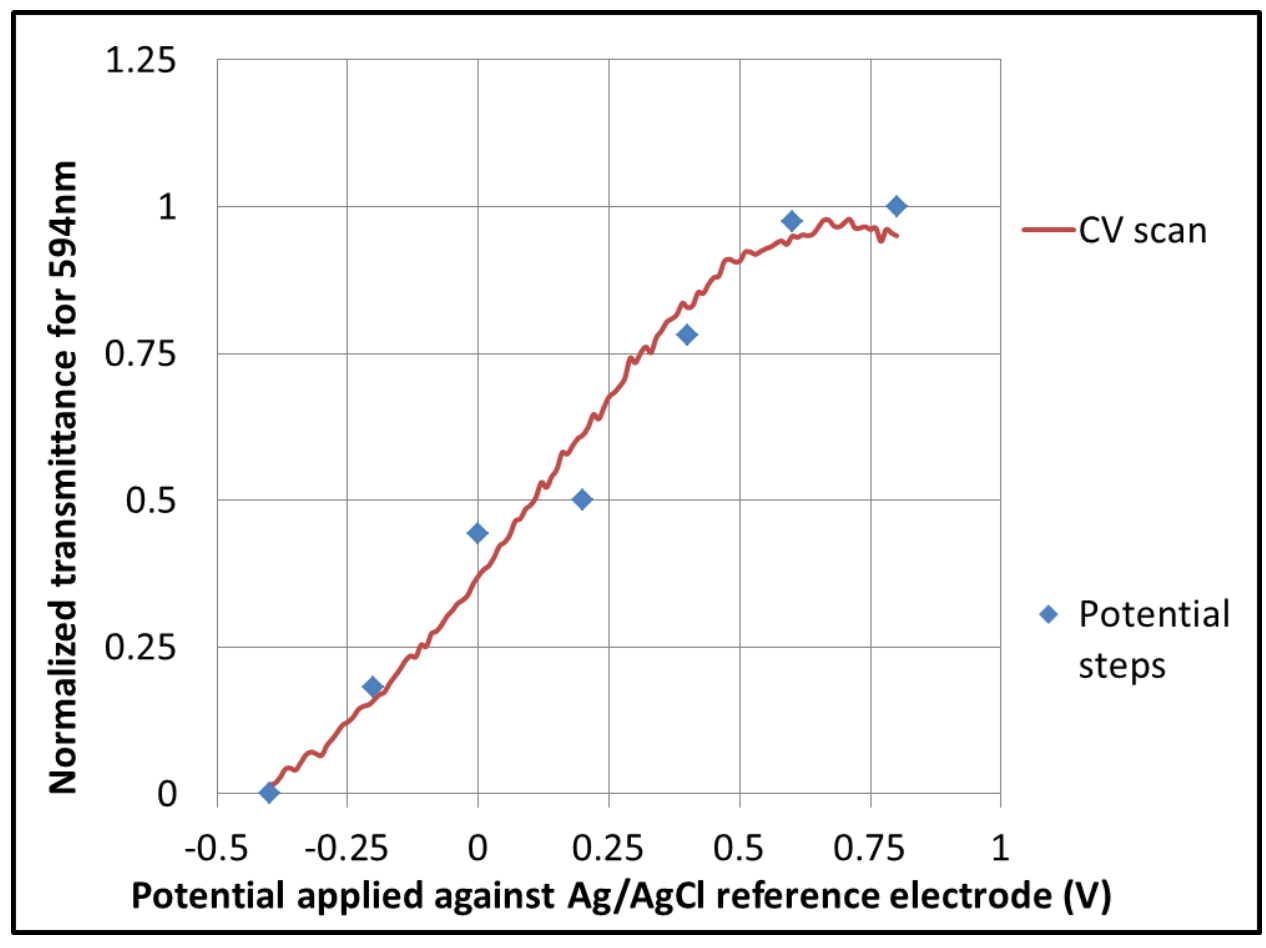

(b)

Figure 3-9: Normalized transmittance comparison between the CV scan and the constant potential steps for (a) $514 \mathrm{~nm}$ and (b) $594 \mathrm{~nm}$.

Impedance with $\mathrm{AC}$ potential modulation

Impedance measurements in the frequency domain with $\mathrm{AC}$ potential modulation were used to examine the ultra-thin ITO film fabricated in the EA-SMIOW. The experiment set-up with the same stationary coupling system was used for these measurements, as shown in Figure 3-10. For the probing light source, a super continuum fiber laser source (Fianium, SC-400-4) was used. An acousto-optical filter was connected to the fiber laser source to select the desired wavelength. The out-coupled intensity was collected by a PMT (Hamamatsu R928) and amplified by a current preamplifier (Stanford Research Systems, SR 570). The AC modulated 
potential signal and the optical out-coupled signal were then collected simultaneously by an oscilloscope (Agilent, DS08104A Infiniium). The electrical impedance results were recorded by the potentiostat. A set of AC impedance potential modulations was applied. Each modulation had a fixed DC bias potential ($0.2 \mathrm{~V}$ to $0.28 \mathrm{~V})$, a constant $\mathrm{AC}$ amplitude $(10 \mathrm{mV})$ and a sequence of oscillation frequencies (1 $\mathrm{Hz}$ to $20 \mathrm{~Hz})$.

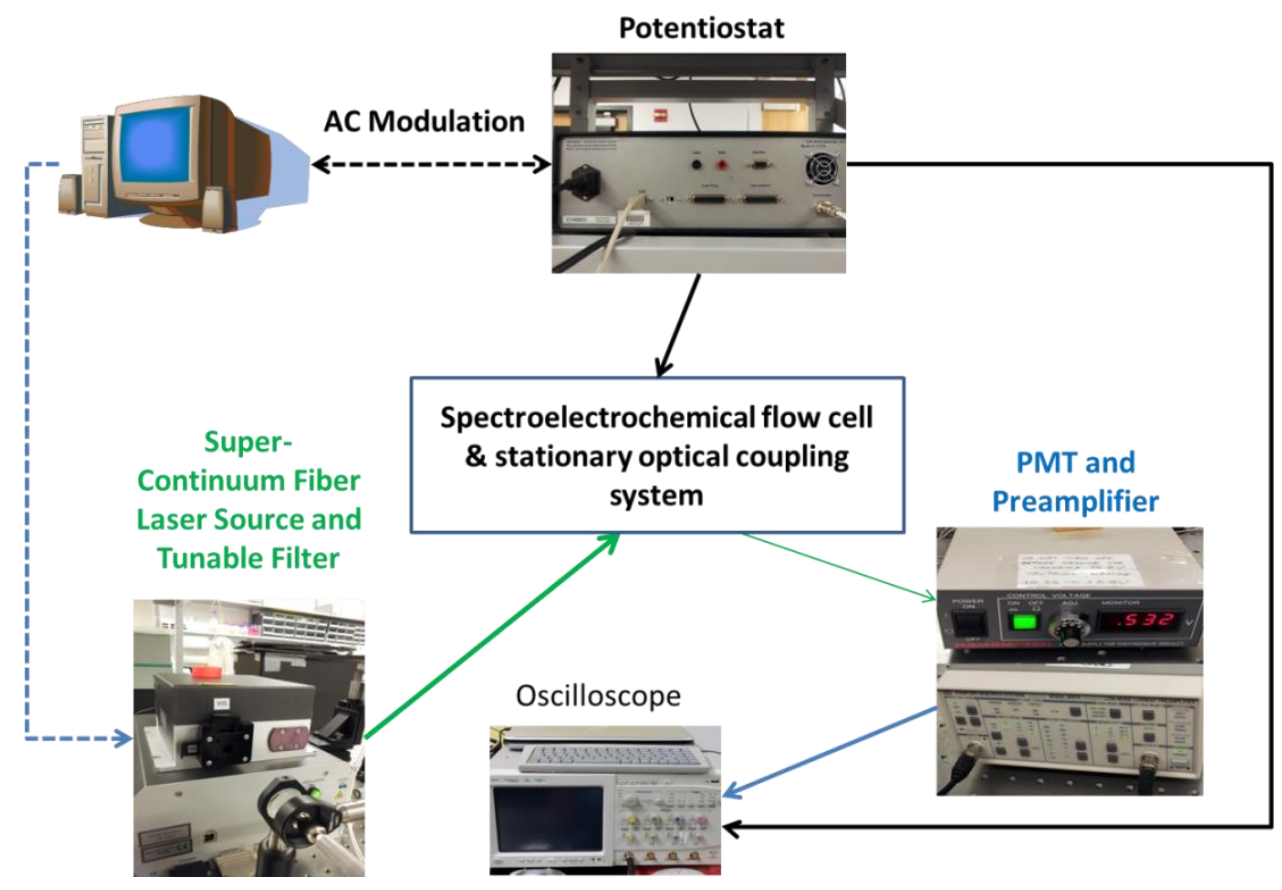

Figure 3-10: Experiment set-up for the AC potential modulation measurement. A super continuum fiber laser source is used as the light source and an acousto-optical filter is used to select probing wavelength. The out-coupled light intensity is collected by a PMT and amplified by a pre-current amplifier. The potential modulation signal and the optical response from the spectroelectrochemical flow cell are recorded simultaneously by an oscilloscope. 
A trace of the AC potential modulation at a DC bias of $-0.8 \mathrm{~V}$ and the corresponding optical signal at $550 \mathrm{~nm}$ are shown in Figure 3-11 (a) and (b), separately. As shown in Figure 3-11 (a), the DC bias and the AC amplitude are constants, while different oscillation frequencies are employed. The responding optical signal has a constant DC component, but its AC amplitude depends on the oscillation frequency. These results demonstrate that the optical response of the ultra-thin ITO film is also frequency dependent. And, these results must be considered for implementing an impedance technique in the EA-SM-IOW platform.

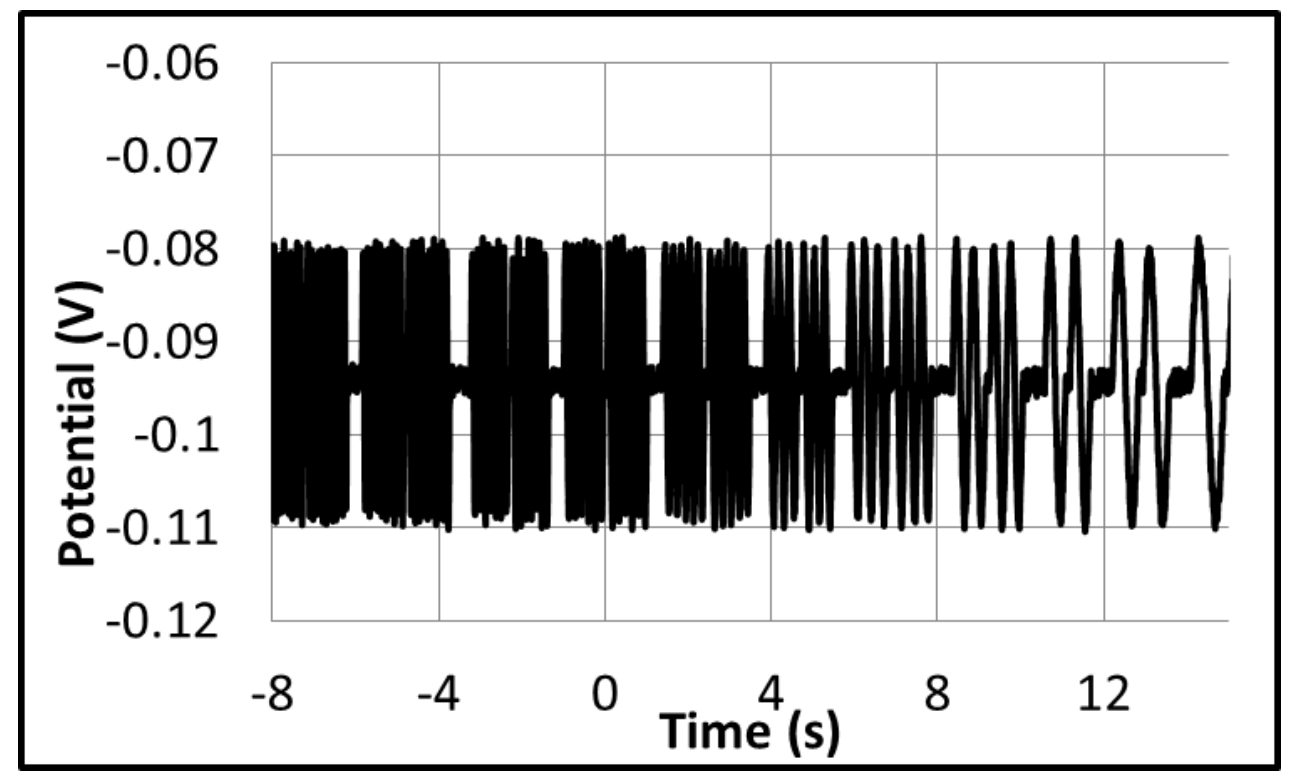

(a) 


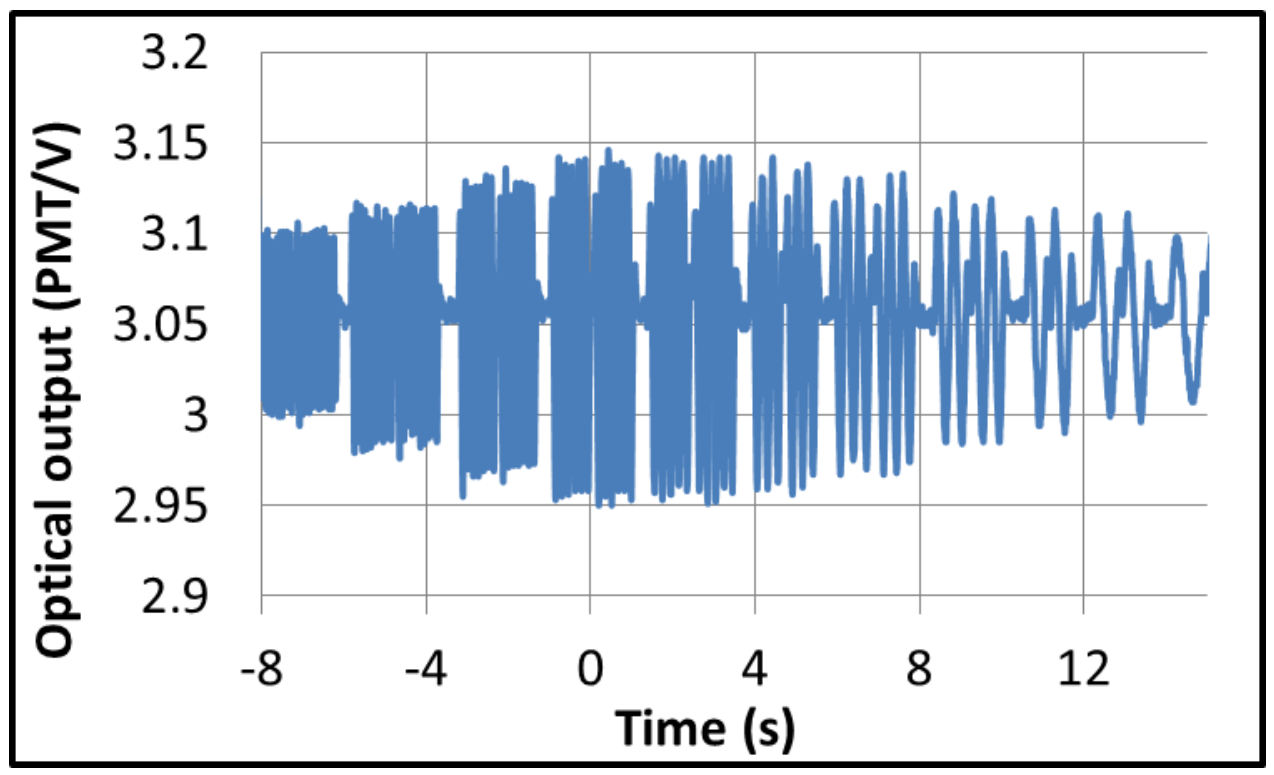

(b)

Figure 3-11: (a) AC potential modulation signal at - $0.8 \mathrm{~V} \mathrm{DC}$ bias and (b) the optical out-coupled intensity signal with $550 \mathrm{~nm}$ probing wavelength.

The expression of the AC impedance potential modulation is shown in Equation 3-2 where $E_{d c}$ is the potential DC bias, $\Delta E_{a c}$ is the AC amplitude, and $\omega$ is the angular frequency of the potential oscillation. Under the condition that the AC amplitude is small enough, the optical response can be expressed with a linear approximation, as in Equation 3-3, where $I_{d c}$ is the DC component, $\Delta I_{a c}$ is the AC amplitude, and angle $\theta$ is the phase delay. A sinusoidal wave fitting process can be carried out to obtain the values of $I_{d c}, \Delta I_{a c}$ and $\theta$.

$$
\begin{aligned}
& E(t)=E_{d c}+\Delta E_{a c} \sin (\omega t) \\
& I(t, \lambda)=I_{d c}\left(E_{d c}, \lambda\right)+\Delta I_{a c}\left(\omega, E_{d c}, \lambda\right) \sin \left[\omega t+\theta\left(\omega, E_{d c}, \lambda\right)\right]
\end{aligned}
$$


Average values of $I_{d c}\left(E_{d c}\right)$ over the oscillation frequency are plotted against $E_{d c}$ for $550 \mathrm{~nm}$ and $580 \mathrm{~nm}$ in Figure 3-12. The standard deviation is set as an error bar for each DC bias potential.

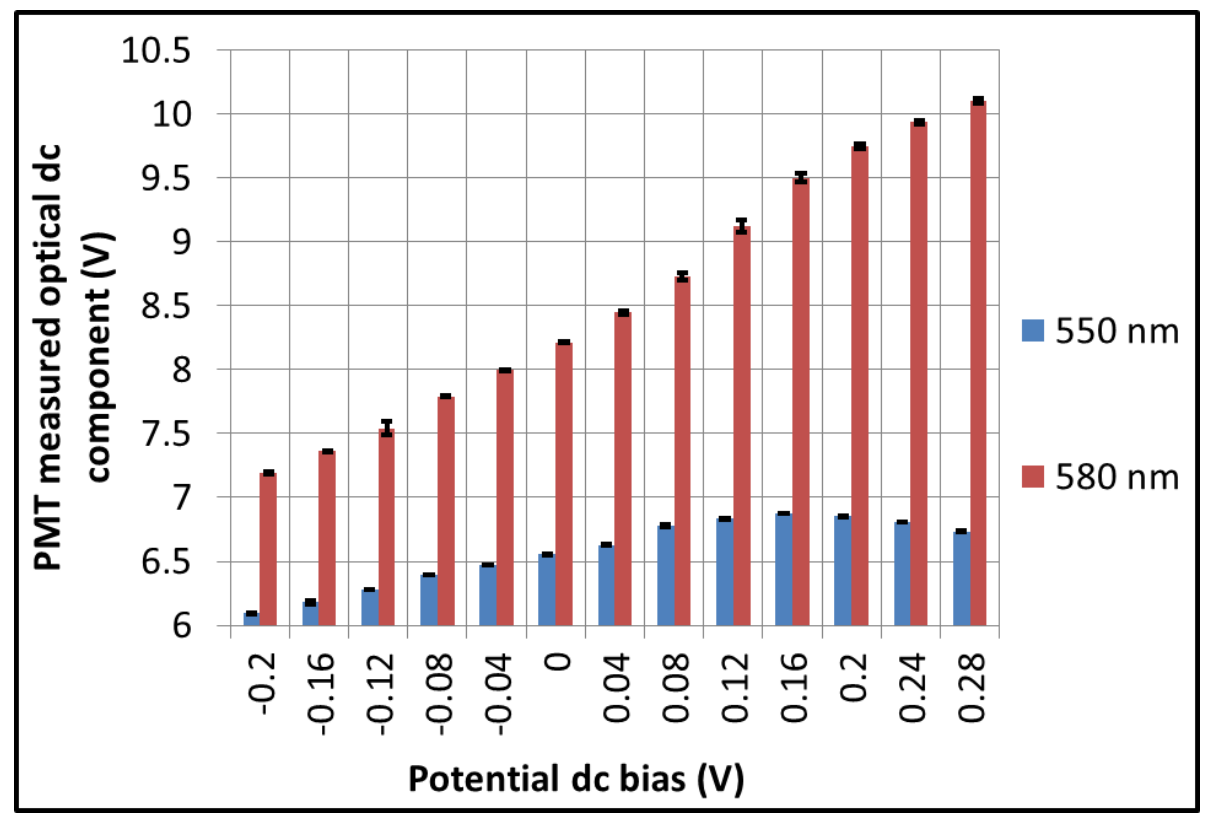

Figure 3-12: Optical DC component intensity for $550 \mathrm{~nm}$ and $580 \mathrm{~nm}$.

The potential DC bias can be interpreted as a constant potential step or the potential point at the slow CV scans. The DC components of the optical signals are compared to the optical response under $\mathrm{CV}$ potential scan. The normalized transmittance curves are shown in Figure 3-13 for (a) $550 \mathrm{~nm}$ and (b) $580 \mathrm{~nm}$ separately. For the CV scan, the normalized transmittance was calculated in the potential range from $-0.2 \mathrm{~V}$ to $+0.28 \mathrm{~V}$. Matches are observed for both probing wavelengths. 


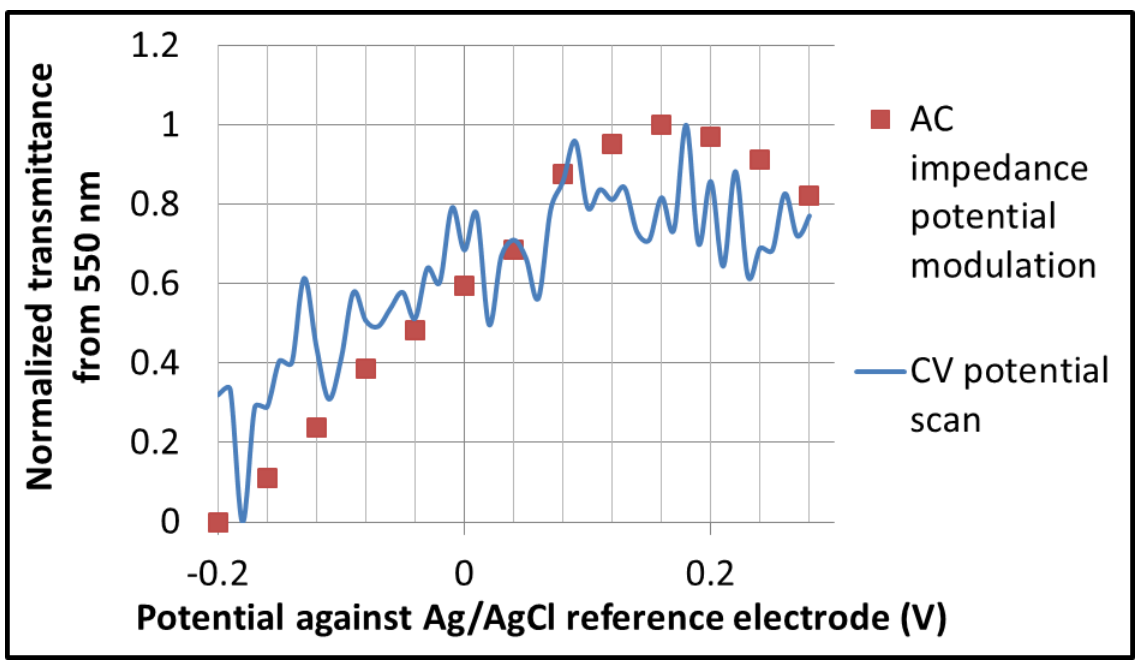

(a)

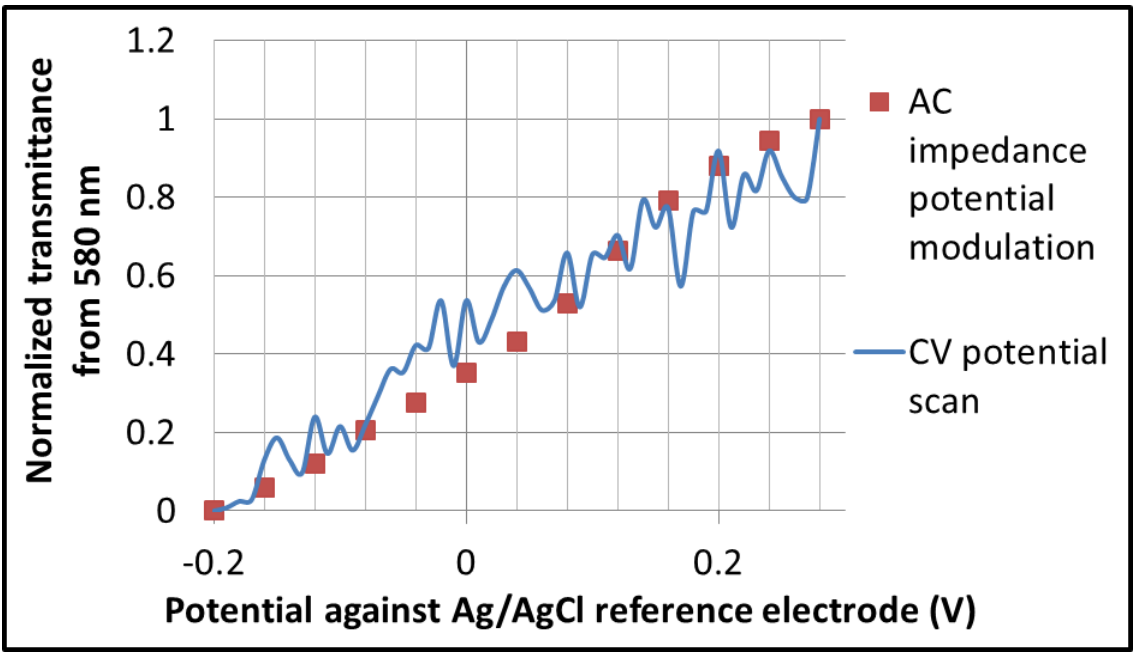

(b)

Figure 3-13: The comparison of normalized transmittance between the optical DC components from the $\mathrm{AC}$ impedance potential modulation and the $\mathrm{CV}$ potential scan used a tungsten-halogen white light source. A match is observed for each probing wavelength (a) $550 \mathrm{~nm}$ and (b) $580 \mathrm{~nm}$.

The optical AC amplitudes are plotted against oscillation frequencies for each potential DC bias, as shown in Figure 3-14. For the $550 \mathrm{~nm}$ light, at $+0.12 \mathrm{~V}$ DC bias, $\Delta I_{a c}$ has the lowest profile. For the $580 \mathrm{~nm}$ light, at $+0.28 \mathrm{~V} \mathrm{DC}$ bias, $\Delta I_{a c}$ has the 
lowest profile. At oscillation frequency of $5 \mathrm{~Hz}, \Delta I_{a c}$ has the largest value for both $550 \mathrm{~nm}$ and $580 \mathrm{~nm}$. The optical AC amplitude is also dependent on the oscillation frequency, and this dependency profile is not related to the wavelength or the potential DC bias.

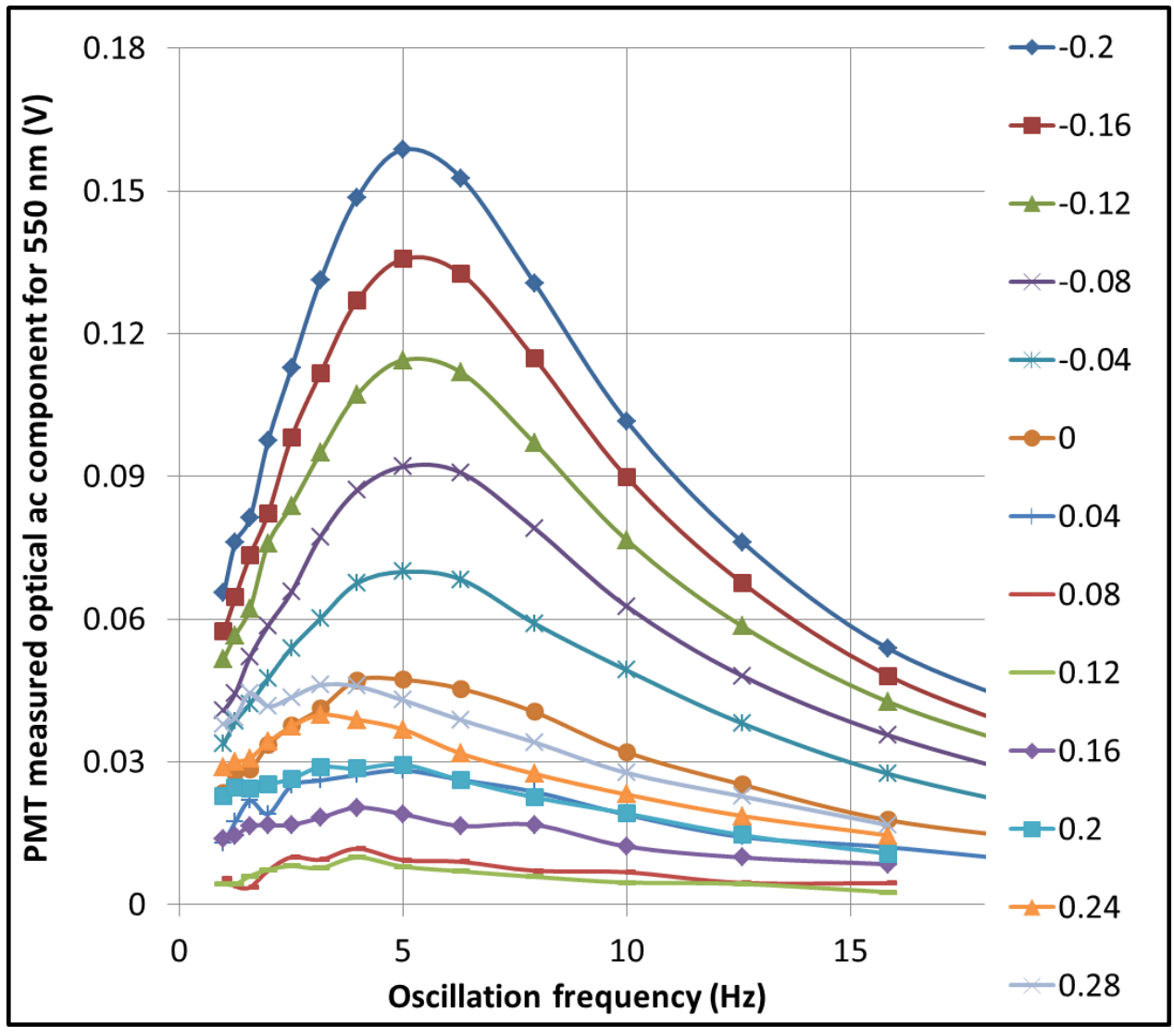

(a) 


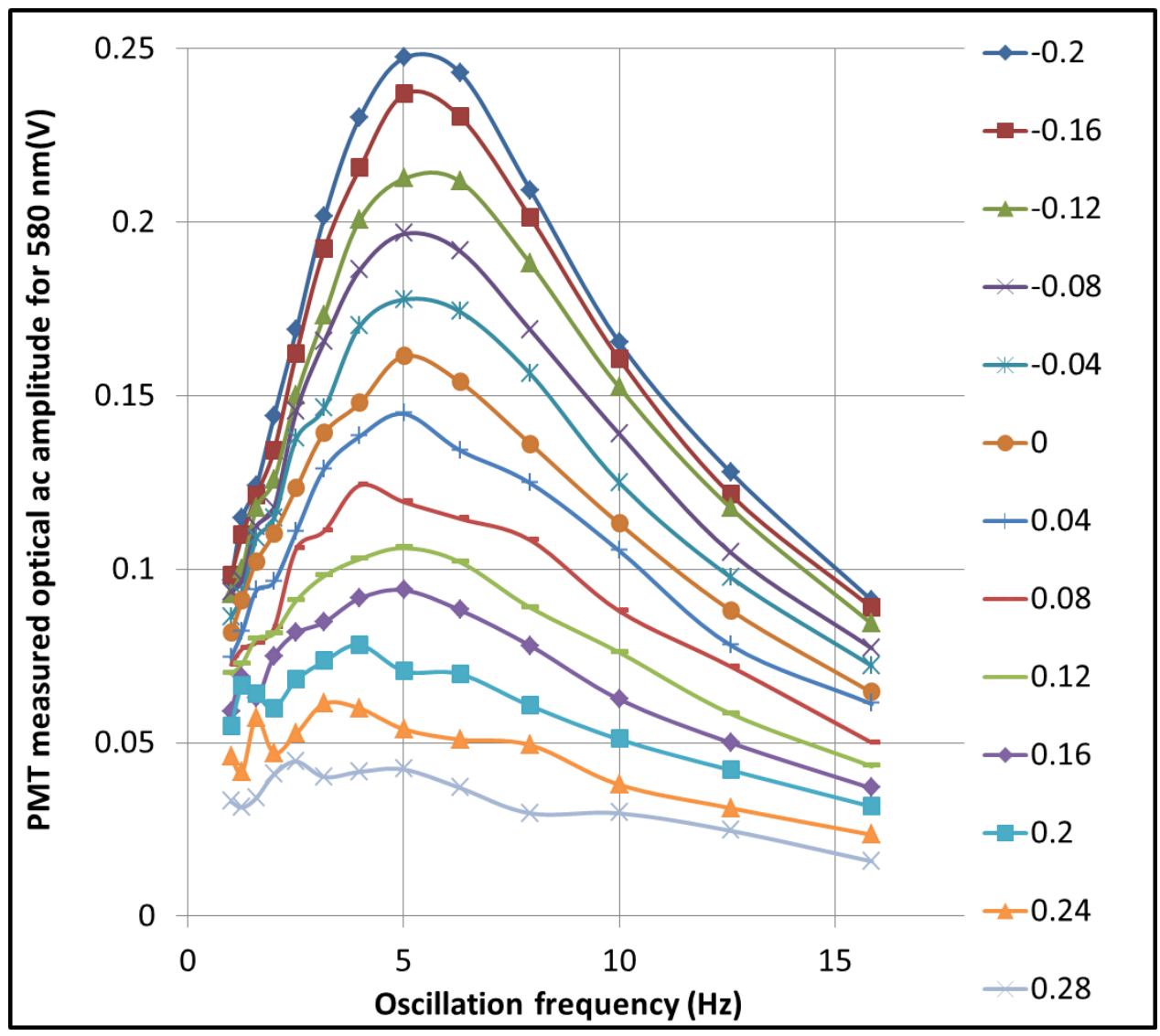

(b)

Figure 3-14: The optical AC amplitude against the potential oscillation frequency for (a) $550 \mathrm{~nm}$ and (b) $580 \mathrm{~nm}$ at different potential DC biases.

The AC components of the optical response in the complex planes (the imaginary component plotted against the real component) are shown in Figure 3-15 for (a) $550 \mathrm{~nm}$ and (b) $580 \mathrm{~nm}$. The potential oscillation frequency has a clockwise direction from $1 \mathrm{~Hz}$ to $20 \mathrm{~Hz}$, as indicated by the black arrows. For the case of 550 $\mathrm{nm}$ probing wavelength, the trace (which is mainly in fourth quadrant) shrinks (magnitude of $\Delta I_{a c}$ decreases) as the potential DC bias increases from $-0.2 \mathrm{~V}$ to + $0.1 \mathrm{~V}$ and then expands again (mainly in the second quadrant). This phenomenon 
could not be observed for the complex plane for $580 \mathrm{~nm}$. Both TE and TM polarized incident light were tested with the ultra-thin ITO films for the AC impedance potential modulations and no difference could be observed (Appendix 6).

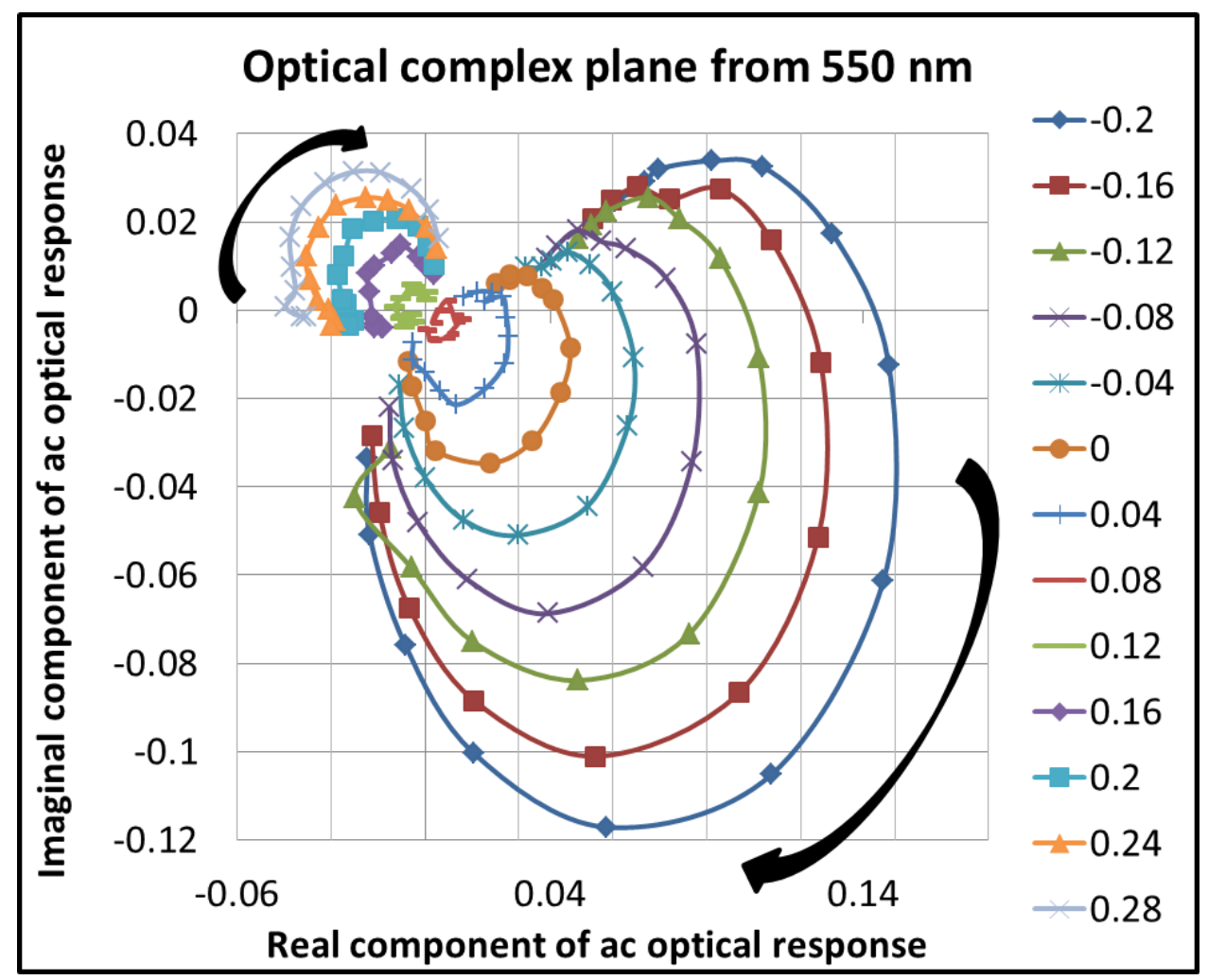

(a) 


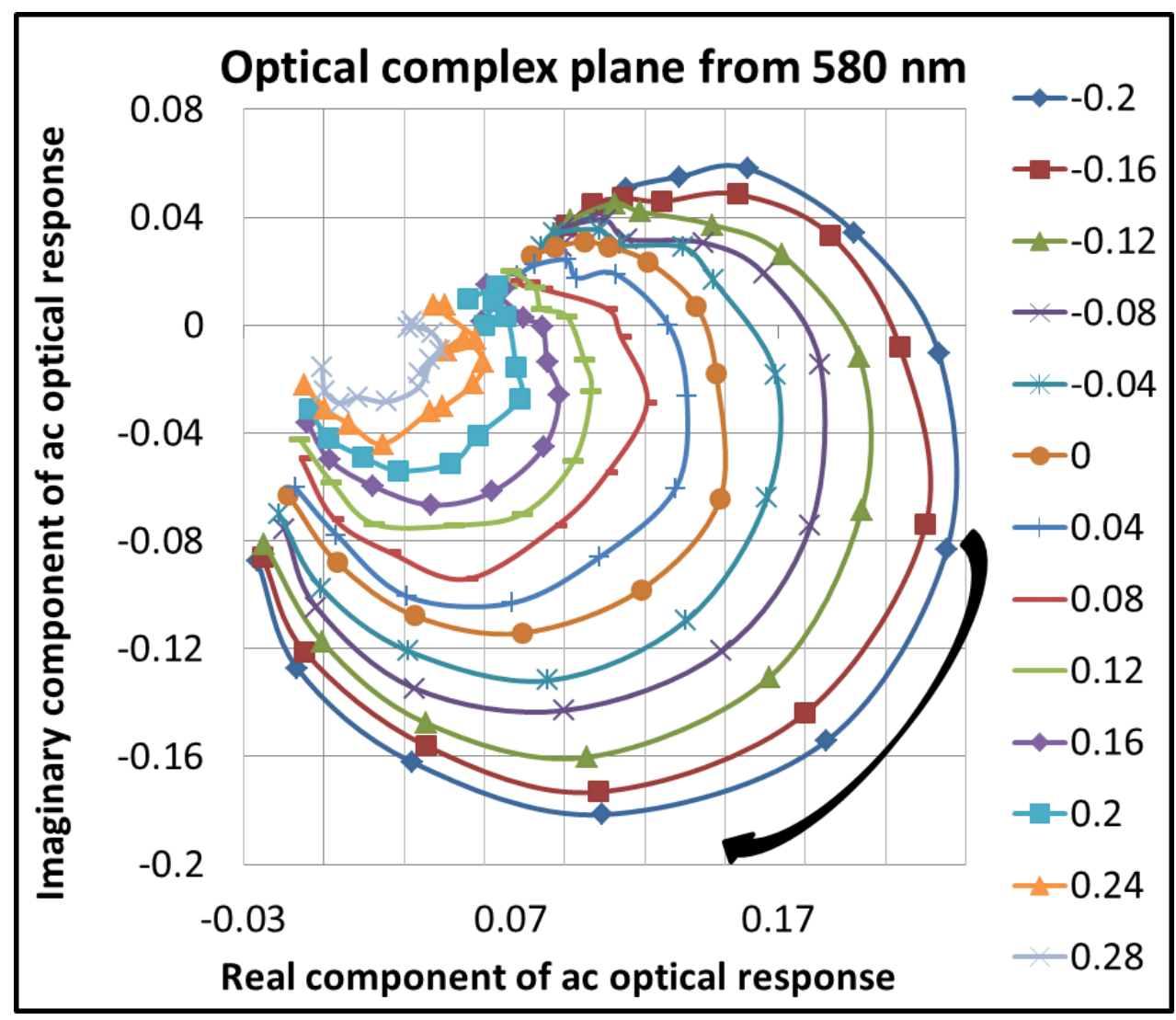

(b)

Figure 3-15: Complex planes from different potential DC biases for (a) $550 \mathrm{~nm}$ and (b) $580 \mathrm{~nm}$.

The electrochemical impedance measurement results measured by the potentiostat are shown in Figure 3-16 for (a) the total impedance amplitude and (b) the phase delay. No evident peaks are observed in the electrical impedance data. 


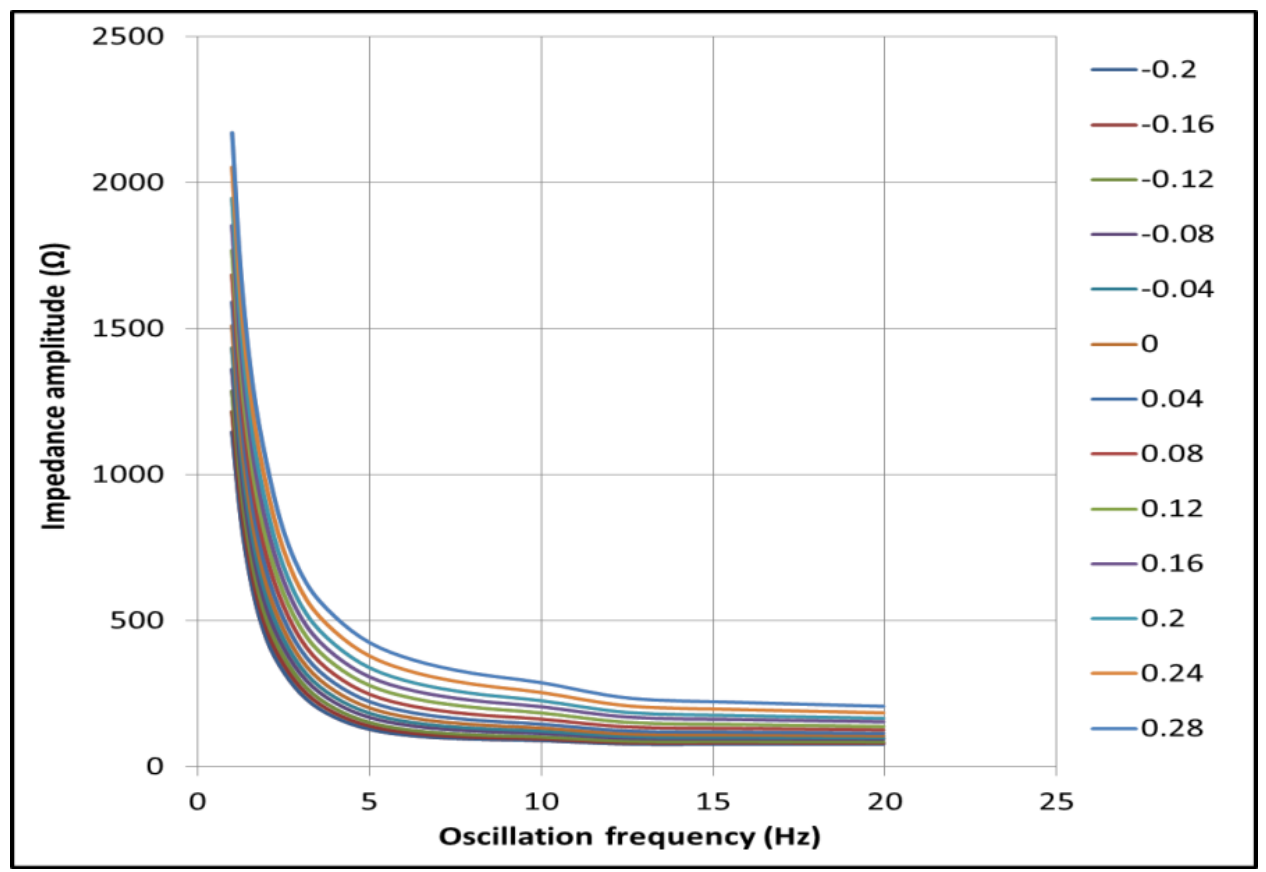

(a)

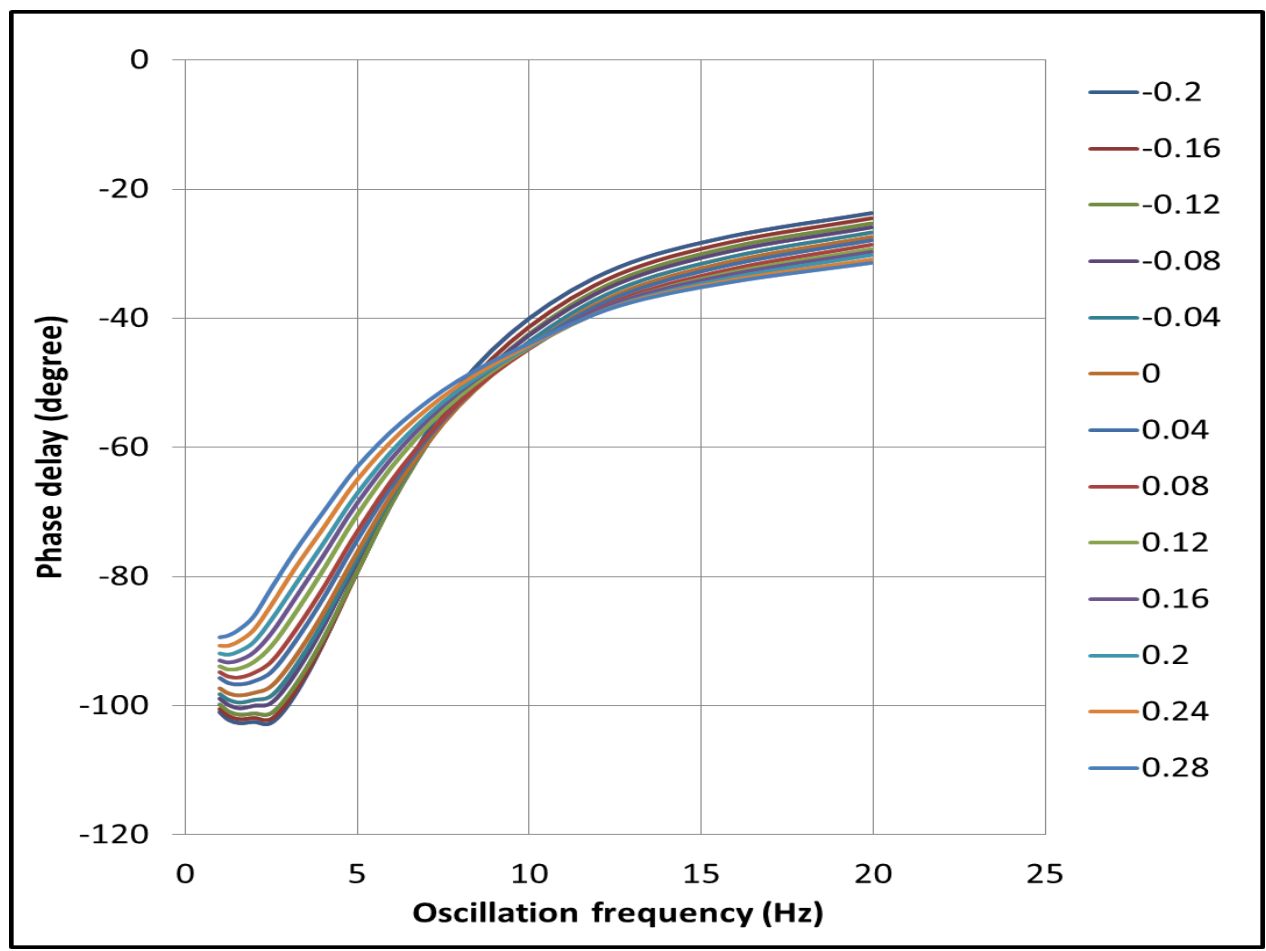

(b)

Figure 3-16: Electrical impedance results of AC potential modulation measured by the potentiostat. (a) The total impedance amplitude and (b) the phase delay against the oscillation frequency. 


\section{Discussions}

Unique spectroscopic features of the ultra-thin ITO film were described in this chapter. The optical properties of an ITO film are related to the energy band structures. They are determined by the ratios of each chemical element indium, tin and oxygen [153]. Due to the doping of the tin element and 0 vacancy, these structures are complicated, especially the band structure corresponding to the visible range.

As the characterization process for ITO films described in Section 2.3, the $\mathrm{O}_{2}$ flow rate during the sputtering process is crucial to the extinction coefficient of the film. For an ultra-thin ITO film, because of its extremely low thickness, $\mathrm{O}_{2}$ in room atmosphere could diffuse into the film to reduce 0 vacancy concentrations and the transparency could be increased.

As the results from Section 3.3.2, the oscillation of the extinction coefficient is the dominant cause of the spectroscopic features of an ultra-thin ITO film under potential modulations. This phenomenon could be attributed to the oscillation of the $\mathrm{O}$ element and $\mathrm{O}$ vacancy concentrations. The $\mathrm{O}$ vacancy could be changed by $\mathrm{Sn}$ element or $\mathrm{H}^{+}$ion diffusion into the film. The thicker ITO (approximately $400 \mathrm{~nm}$ ) films were tested with acid solution under slow CV potential scans. With low $\mathrm{pH}$ value 4.5 solutions, the ITO samples with low $\mathrm{O}_{2}$ flow rate $(0.55 \mathrm{sccm}$ and $0.6 \mathrm{sccm})$ were darkened in the area immersed in the solution, as shown in Figure 3-17. The sample with $0.7 \mathrm{sccm} \mathrm{O}_{2}$ flow rate was not darkened. Research has been done to apply extremely high electrical field (approximately $10^{6} \mathrm{~V} / \mathrm{cm}$ ) on the thick ITO 
films. With the negative potential, the film was darkened and with the positive potential, the film resulted higher 0 element concentration [177].

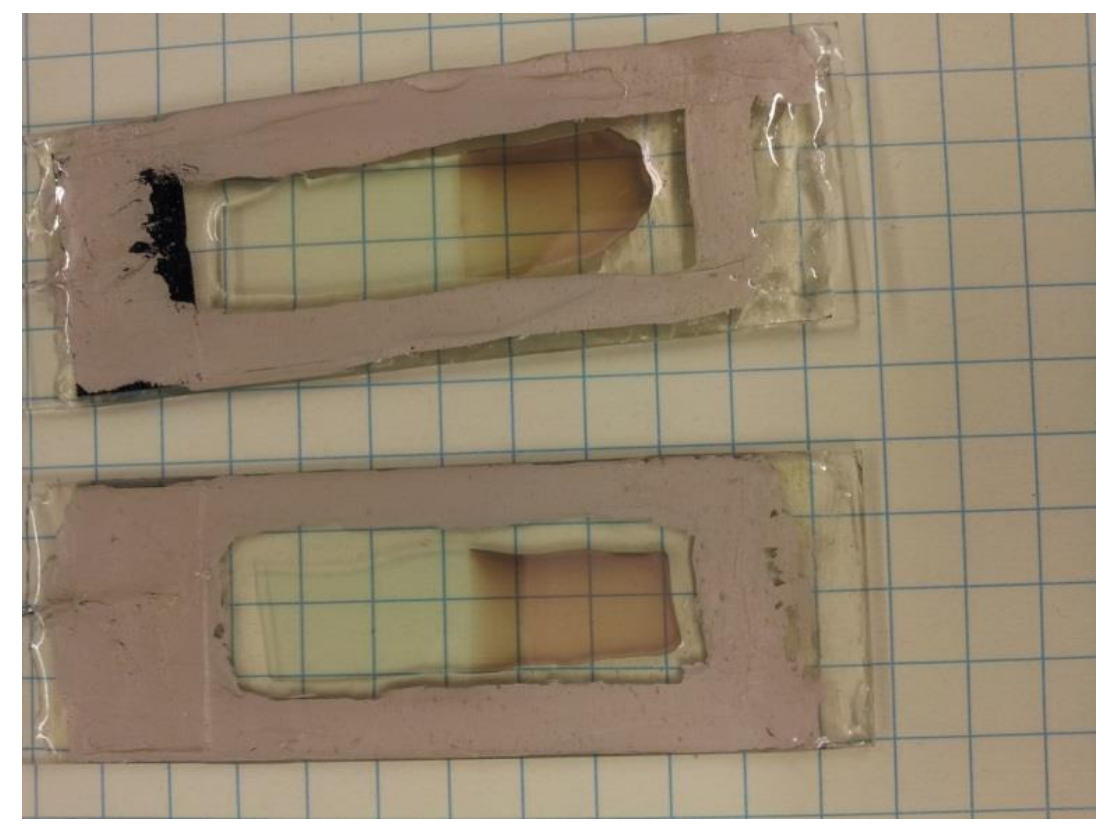

Figure 3-17: Picture of thicker ITO films under a CV potential modulation in $4.5 \mathrm{pH}$ acid solution for sputtered sample with $0.55 \mathrm{sccm}$ and $0.6 \mathrm{sccm}$.

Transparencies of thicker ITO films were tested under the same CV potential scans with a spectrophotometer. No significant change of transmitted intensity could be detected. The reason could be that transmittance measurement is not sensitive enough to show the change of extinction coefficient from the films. The optical probing length was only the thickness of the ITO films. Another reason could be that the potential modulation effects on these thick ITO films are not the same as the ultra-thin ITO films. 


\section{Conclusions}

Spectroscopic features from an ultra-thin ITO film under potential modulations were studied by using the extremely sensitive SM-IOW platform. In the spectral region from $500 \mathrm{~nm}$ to $600 \mathrm{~nm}, \mathrm{CV}$ potential scans, constant potential steps, and AC impedance potential modulations were performed. The optical responses of an ultra-thin ITO film from these three techniques have shown similar results. For the study in spectroelectrochemical behavior of surface-adsorbed redox species based on an EA-SM-IOW platform, these optical signals investigated here represent the baselines. Therefore these features in the optical reference signal under the potential modulation should be factored out to achieve accurate information in electron transfer processes of the redox molecules. 


\section{CHAPTER 4}

\section{APPLICATIONS OF THE ELECTRO-ACTIVE, SINGLE-MODE, INTEGRATED OPTICAL WAVEGUIDE FOR STUDYING REDOX REACTIONS UNDER CYCLIC VOLTAMMETRY}

In this chapter, the application of the electro-active single-mode integrated optical waveguide (EA-SM-IOW) in studying adsorbates of redox species under the cyclic voltammetry (CV) are described. Comparing to the conventional electrochemical CV technique, its high detection sensitivity is demonstrated.

\section{Cytochrome c Protein}

The tested redox protein is cytochrome c, which is a globular protein with well characterized structural and spectroscopic properties in solution environments. The size of this protein is approximately $2.5 \mathrm{~nm} \times 2.5 \mathrm{~nm} \times 3.7 \mathrm{~nm}$. It has multiple functions in different bio-systems, and one of them is to transfer electrons between cytochrome c oxidases [178-185] and reductase complexes in the respiratory system [186-192]. Oxidized cytochrome $\mathrm{c}$ is characterized by the iron atom at +3 state (ferric) inside the heme group, and reduced cytochrome $\mathrm{c}$ has iron atom at +2

state (ferrous). Cytochrome $\mathrm{c}$ has an isoelectric point at $\mathrm{pH}$ value from 10 to 10.5 , 
which makes it carry positive charges in near neutral pH solutions $[193,194]$. They can be immobilized on negatively charged surfaces, such as phospholipid membranes, tin oxide [195], silicon dioxide, gold, and indium tin oxide by electrostatic forces [196]. Assuming that the protein molecules do not have any substantial conformational change during the adsorption process, the maximum surface packing density should be in the range from $17 \times 10^{-12} \mathrm{~mol} / \mathrm{cm}^{2}$ to $26 \times 10^{-12} \mathrm{~mol} / \mathrm{cm}^{2}$, depending on the orientation of the proteins.

Originally oxidized cytochrome c from the horse heart was purchased from Sigma Aldrich with $99.7 \%$ purity. The stock solution had $1 \mu \mathrm{M}$ concentration of cytochrome $\mathrm{c}$ in the phosphate buffer solution $\left(\mathrm{Na}_{2} \mathrm{HPO}_{4}, 5 \mathrm{mM}, \mathrm{pH}=7\right)$.

Molar absorptivity of cytochrome c protein

A conventional spectrophotometer was used. Signal from the buffer solution was used as the reference. The absorbance of $0.33 \mu \mathrm{M}$ originally oxidized cytochrome c was measured first. Then chemical reducing agent $\mathrm{Na}_{2} \mathrm{~S}_{2} \mathrm{O}_{4}$ was placed into the cuvette to reduce cytochrome c proteins. The absorbance of the reduced cytochrome c was measured. The $\mathrm{Na}_{2} \mathrm{~S}_{2} \mathrm{O}_{4}$ compound added no absorbance in the spectral range under consideration. The molar absorptivity for both redox states was calibrated, as shown in Figure 4-1. The characteristics absorbance peaks (409 $\mathrm{nm}$ and $528 \mathrm{~nm}$ ) for the oxidized state, and (416 $\mathrm{nm}$ and $550 \mathrm{~nm})$ the reduced state of cytochrome c proteins in a solution environment were observed [197]. Isosbestic wavelengths (reduced and oxidized states of cytochrome c protein have the same molar absorptivity) were also observed at $541 \mathrm{~nm}$ and $557 \mathrm{~nm}$. In the solution 
environment, $100 \%$ of cytochrome c proteins were chemically reduced based on the absorbance results.

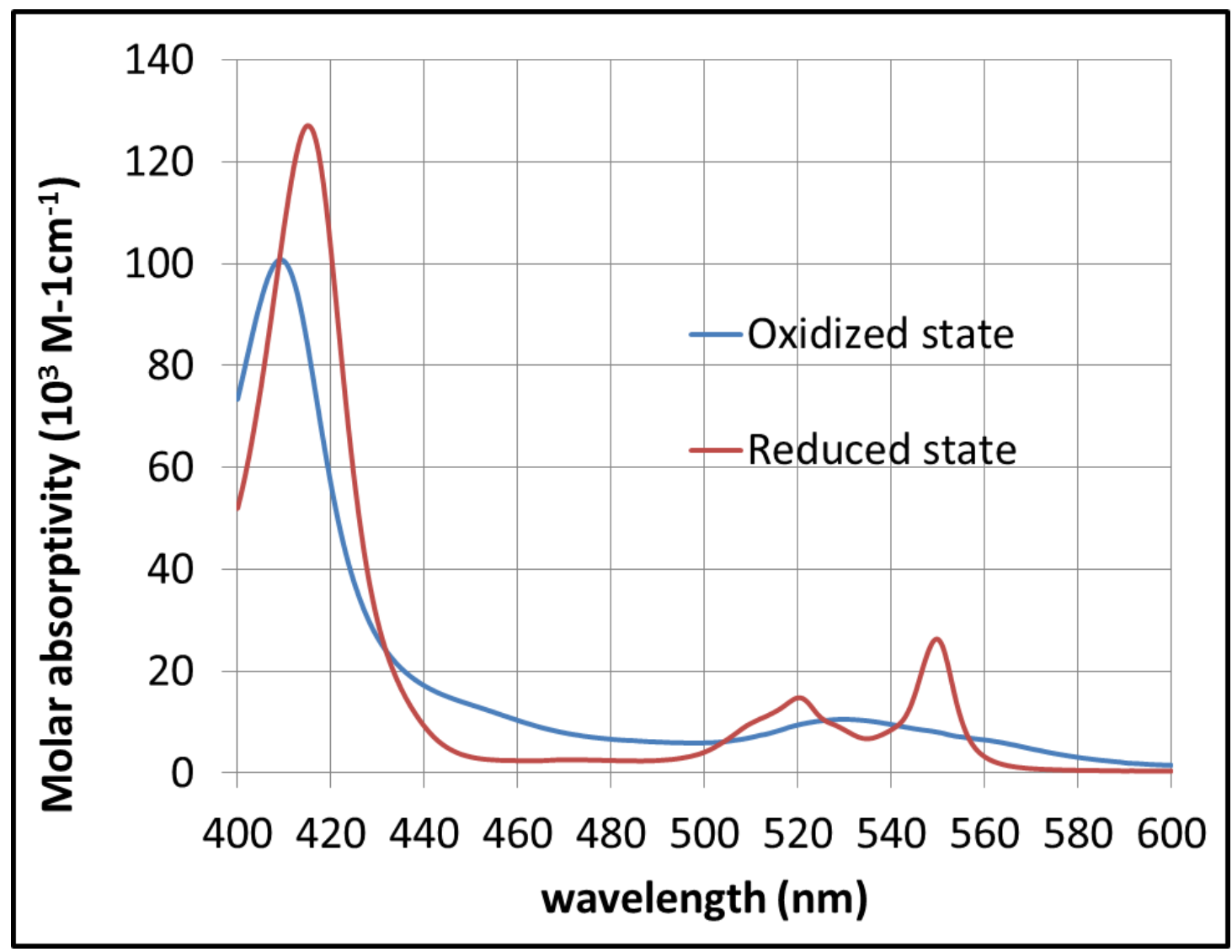

Figure 4-1: Calibrated molar absorptivity for the oxidized and the reduced states of cytochrome c proteins in buffer solution.

For the application of the EA-SM-IOW, the faradaic current of cytochrome c proteins can be reconstructed by using signal from $550 \mathrm{~nm}$ that shows a strong molar absorptivity difference. The total surface coverage of cytochrome c proteins 
can be monitored during the adsorption processes and CV scans by using the signal from the isosbestic wavelength of $557 \mathrm{~nm}$.

\section{Experimental Processes and Results}

\section{Experimental procedures}

The potential difference between the pseudo $\mathrm{Ag} / \mathrm{AgCl}$ reference electrode and the standard $\mathrm{Ag} / \mathrm{AgCl}$ reference electrode was calibrated (Appendix 7). The potential difference was $-0.0852 \mathrm{~V}$. The experimental results for the electrochemical reactions in this dissertation were all in the reference of the pseudo $\mathrm{Ag} / \mathrm{AgCl}$ reference electrode. The same experiment set-up with the stationary coupling system as in Figure 3-2 was used. The CV potential scan was set from - 0.4 $\mathrm{V}$ to $+0.4 \mathrm{~V}$ at a speed of $0.02 \mathrm{~V} / \mathrm{s}$. A super-continuum fiber laser was used as a broadband light source. For the optical data collection, the ICCD camera connected by a monochromator was used. The internal trigger of the camera was $2 \mathrm{~Hz}$, and exposure time for each frame was $200 \mathrm{~ms}$.

The optical and electrical reference signals were collected first without the presence of cytochrome c proteins in the flow cell. Then the system was relaxed for about 30 minutes. After the relaxation, $2 \mathrm{ml}$ of $100 \mathrm{nM}$ cytochrome c solution was injected into the flow cell. Another 30 minutes was used to stabilize the adsorption process of cytochrome c proteins on top of the ITO surface. Then the flow cell was flushed with a buffer solution to eliminate unattached cytochrome c proteins. The same $\mathrm{CV}$ potential scan was applied to the flow cell with cytochrome c presence, and 
optical signal was recorded. After all the redox reaction measurements, a black ink solution was injected into the flow cell and one frame of the dark background was measured under the same exposure time (200 ms).

\section{Absorbance signal}

The $\mathrm{CV}$ potential scan and the optical signals, at $550 \mathrm{~nm}$ for the reference and for the surface-adsorbed proteins are plotted against time, as shown in Figure 4-2 (a), (b), (c) separately. As previously discussed, the optical reference signal is dependent on the potential modulation, and those effects need to be factored out for the protein absorbance calculation.

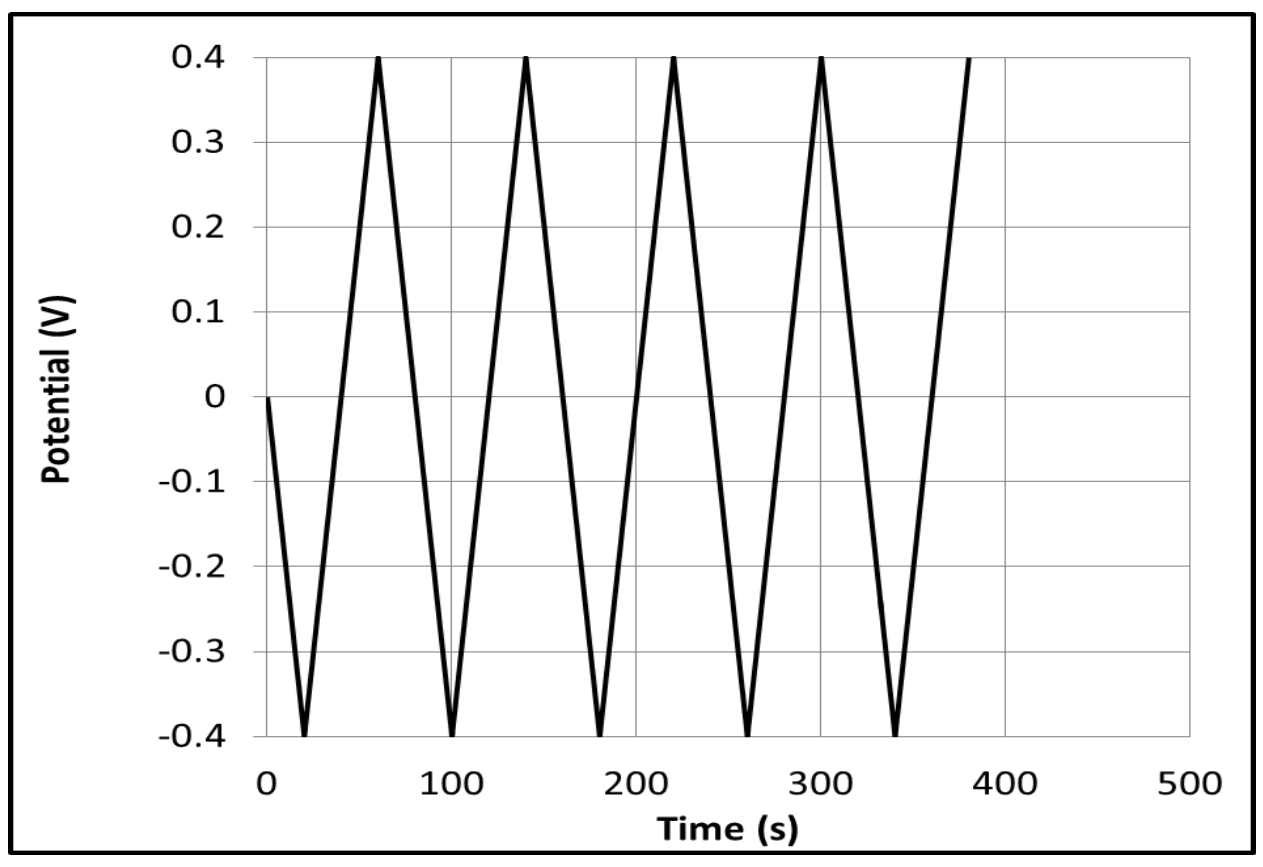

(a) 


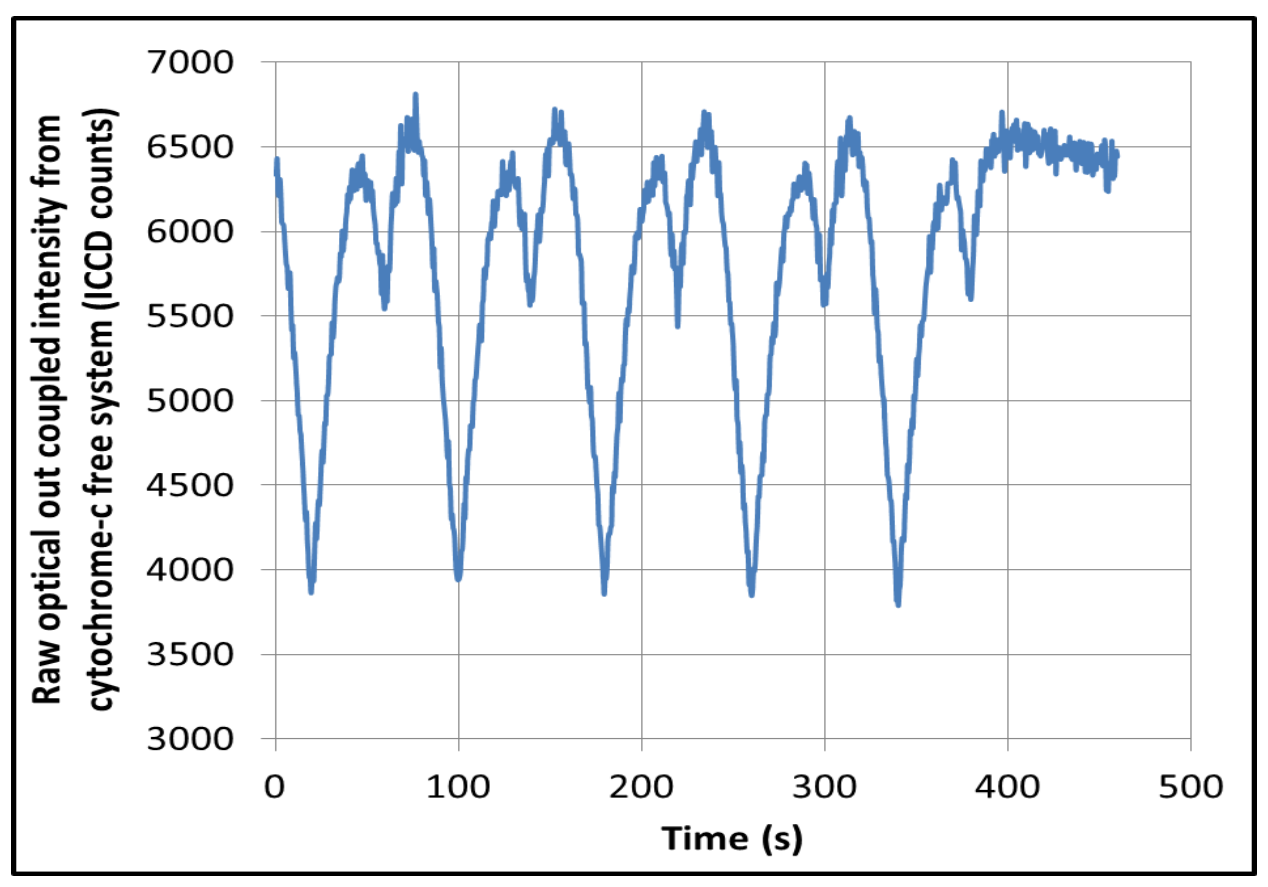

(b)

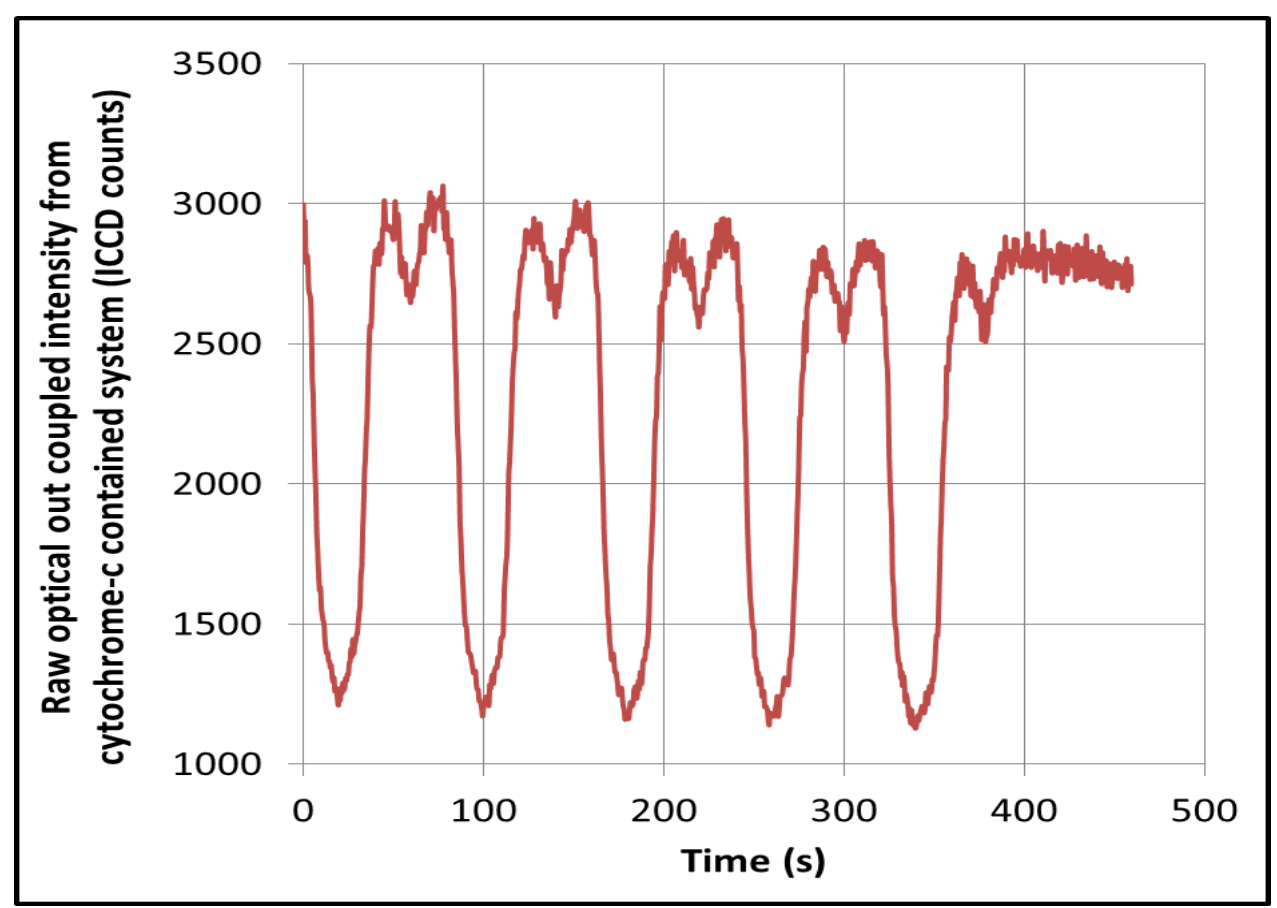

(c)

Figure 4-2: (a) CV potential scan of 5 cycles between $-0.4 \mathrm{~V}$ and $+0.4 \mathrm{~V}$ with 0.02 $\mathrm{V} / \mathrm{s}$ speed, (b) the optical reference signal, and (c) the optical signal from 
cytochrome c proteins contained system under the same CV potential scan from 550 nm probing wavelength.

Experimental optical raw signals from the reference system and the cytochrome c contained system were used to generate the absorbance data by using the point by point method. The general expression of absorbance $A b s(E)$ can be written in Equation 4-1, where $E$ is the applied potential, $I(E)$ is the out-coupled optical intensity from the cytochrome c contained system, $I_{0}(E)$ is the reference optical signal, and $I_{d a r k}$ is the dark background intensity.

$$
\operatorname{Abs}(E)=-\log _{10}\left(\frac{I(E)-I_{\text {dark }}}{I_{0}(E)-I_{\text {dark }}}\right)
$$

This absorbance result is directly related to the amount of surface adsorbed proteins and their redox states, as shown in Equation 4-2, where $\Gamma_{\text {tot }}$ is the total surface coverage, $\Gamma_{\text {act }}$ is the active surface coverage of cytochrome c proteins under the $\mathrm{CV}$ potential scan, $\Delta \epsilon$ is the molar absorptivity difference between the reduced and the oxidized states, $\epsilon_{o x}$ is the molar absorptivity of the proteins at the oxidized state, and $f(E)$ is the fraction of the active cytochrome c proteins that are at reduced state at applied potential, $E$. The fraction function $f(E)$ varies between 0 and 1 during the potential scan. If the total surface coverage of cytochrome c proteins immobilized on the surface are all active, $\Gamma_{a c t}$ equals to $\Gamma_{\text {tot }}$. The sensitivity factor, $S$, was determined theoretically in section 2.4.4 and experimentally in 2.4.5.

$$
A b s(E)=S \Gamma_{a c t} \Delta \epsilon f(E)+S \Gamma_{\text {tot }} \epsilon_{o x}
$$


The absorbance results for three wavelengths, $550 \mathrm{~nm}, 557 \mathrm{~nm}$ and $580 \mathrm{~nm}$, are shown in Figure 4-3.

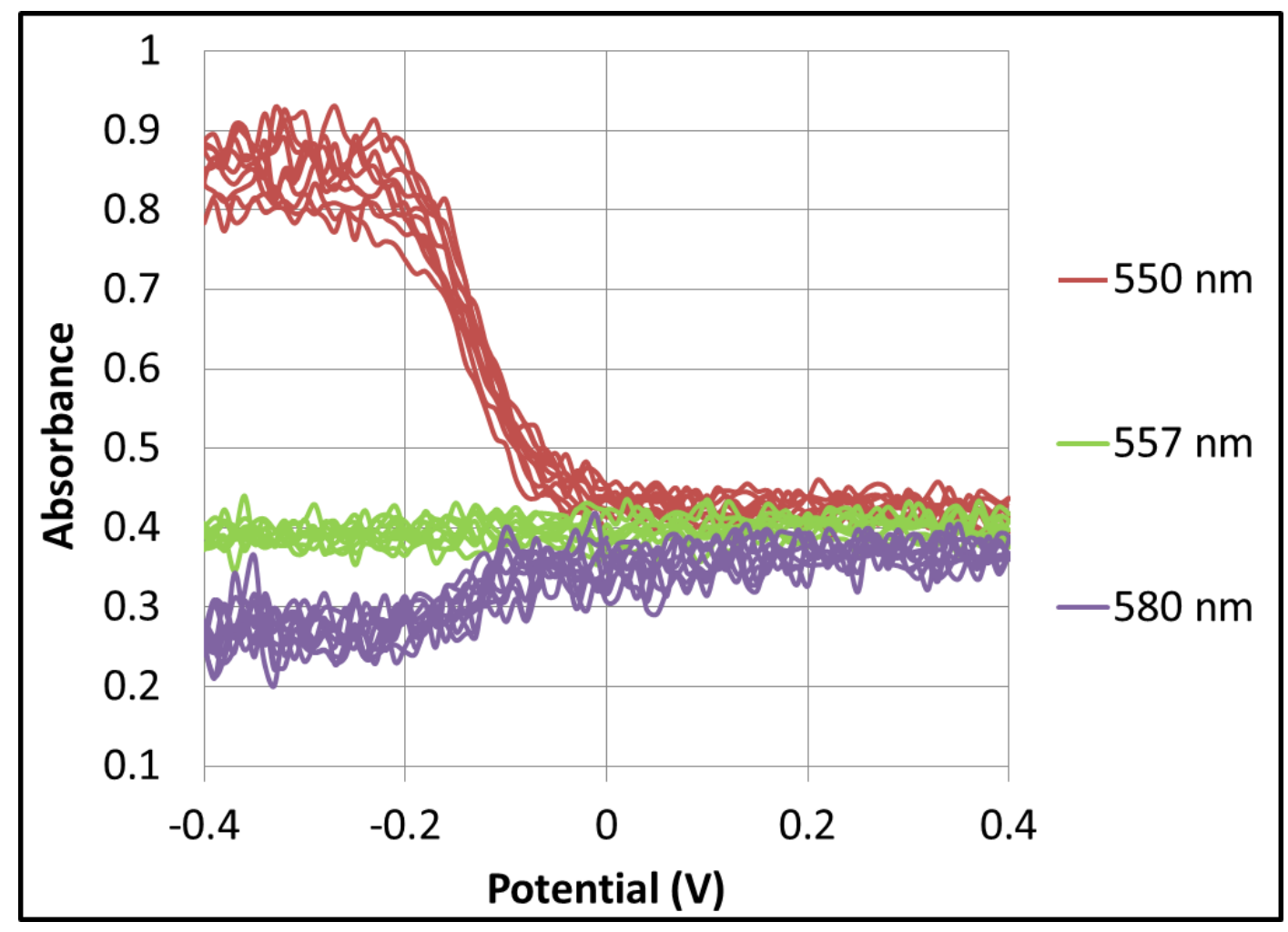

Figure 4-3: Absorbance curves from three wavelengths $550 \mathrm{~nm}, 557 \mathrm{~nm}$ and $580 \mathrm{~nm}$ for cytochrome c proteins under a CV potential scan.

By examining the absorbance curve from an isosbestic point $557 \mathrm{~nm}$, it could be concluded that there was no adsorption nor desorption during the potential scanning process. Because of $\epsilon_{o x}(557 \mathrm{~nm})=\epsilon_{\text {red }}(557 \mathrm{~nm})=\epsilon$, Equation 4-2 can be modified for the total surface coverage calculation as Equation 4-3. It is also can be 
calculated by using the absorbance data from $550 \mathrm{~nm}$ at the positive potential range before the redox reaction happening because that $f(E)$ is 0 in this case.

$$
\begin{aligned}
& \overline{A b s(E)}=S \Gamma_{\text {tot }} \epsilon(557 \mathrm{~nm}) \\
& \overline{A b s(E)}=S \Gamma_{\text {tot }} \epsilon_{o x}(550 \mathrm{~nm})
\end{aligned}
$$

The calculated $\Gamma_{\text {tot }}$ were $(3.6 \pm 0.1)$ picomoles $/ \mathrm{cm}^{2}$ for $550 \mathrm{~nm}$ and $(4.0 \pm 0.2)$ picomoles $/ \mathrm{cm}^{2}$ for $557 \mathrm{~nm}$. Under the experimental conditions described above, $\Gamma_{\text {tot }}$ was about $18 \%$ of a full monolayer.

At $550 \mathrm{~nm}, \Delta \epsilon(550 \mathrm{~nm})>0$, the reduced cytochrome c proteins absorb more light than the oxidized ones, and a higher absorbance is resulted at reducing potentials. As shown by the red curve in Figure 4-3, the absorbance from $550 \mathrm{~nm}$ increases when the potential scanned towards negative values and reaches a maximum when all active species are reduced. At $580 \mathrm{~nm}, \Delta \epsilon(580 \mathrm{~nm})<0$, the reduced cytochrome c proteins absorb less light. The opposite change of absorbance is observed.

\section{Reconstruction of faradaic current density}

The absorbance against potential curve from $550 \mathrm{~nm}$ was used to reconstruct the faradaic current density, $i_{F}(E)$, with Equation $4-4$, where $n$ is the number of the electron transferred for one redox reaction (ideally equals to unit for the cytochrome c), $F$ is the faraday constant, $v$ is the $\mathrm{CV}$ potential scan speed. Figure $4-4$ shows the reconstructed faradaic current density for a folded 5 -cycles (10 segments) CV scan. Both positive and negative potential scan directions are used. 


$$
i_{F}(E)=n F \frac{d \Gamma_{r e d}(E)}{d t}=-n F \frac{d \Gamma_{o x}(E)}{d t}=n F \frac{d A b s(E)}{S \Delta \epsilon d t}=n F \frac{d A b s(E)}{S \Delta \epsilon d E} v
$$

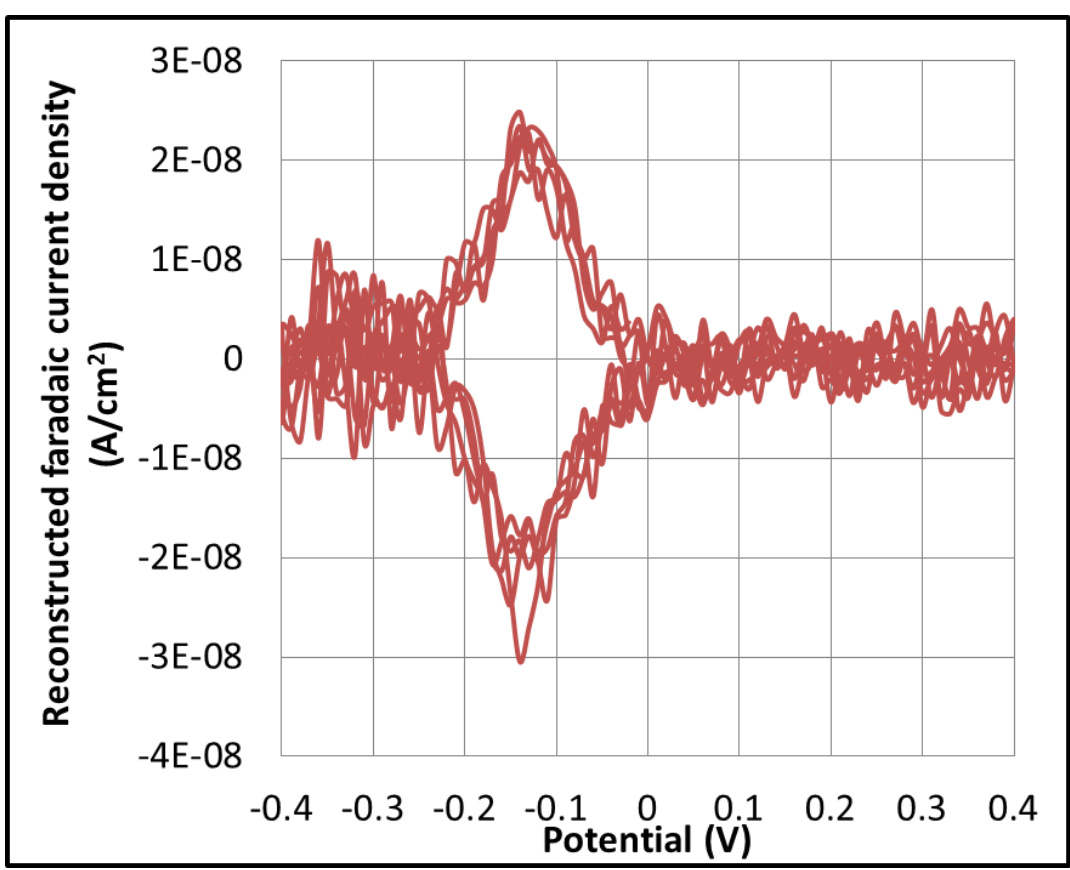

Figure 4-4: The 5-cycle of faradaic current density reconstructed by the optical absorbance signal from $550 \mathrm{~nm}$. Both potential scan directions are included.

There is no separation of the anodic and cathodic faradaic current density peaks, and the peaks have a similar value. The electron transfer process between the working electrode (ITO layer) and cytochrome c proteins can be considered as a reversible process.

A Gaussian fit was used to examine the properties of faradaic current density profile. Each segment of the 5-cycle CV scans was fitted separately. The average of those fitting results for the formal potential is $E_{0}=(-0.131 \pm 0.003) V$, the peak 
current density is $i_{p}=\left(2.2 \pm 0.3 \times 10^{-8}\right) \mathrm{A} / \mathrm{cm}^{2}$, and the full width at half maximum (FWHM) is $\Delta E_{1 / 2}=(-0.08 \pm 0.02) V$. In Figure 4-5, one segment of the faradaic current density profile is shown with its Gaussian fit.

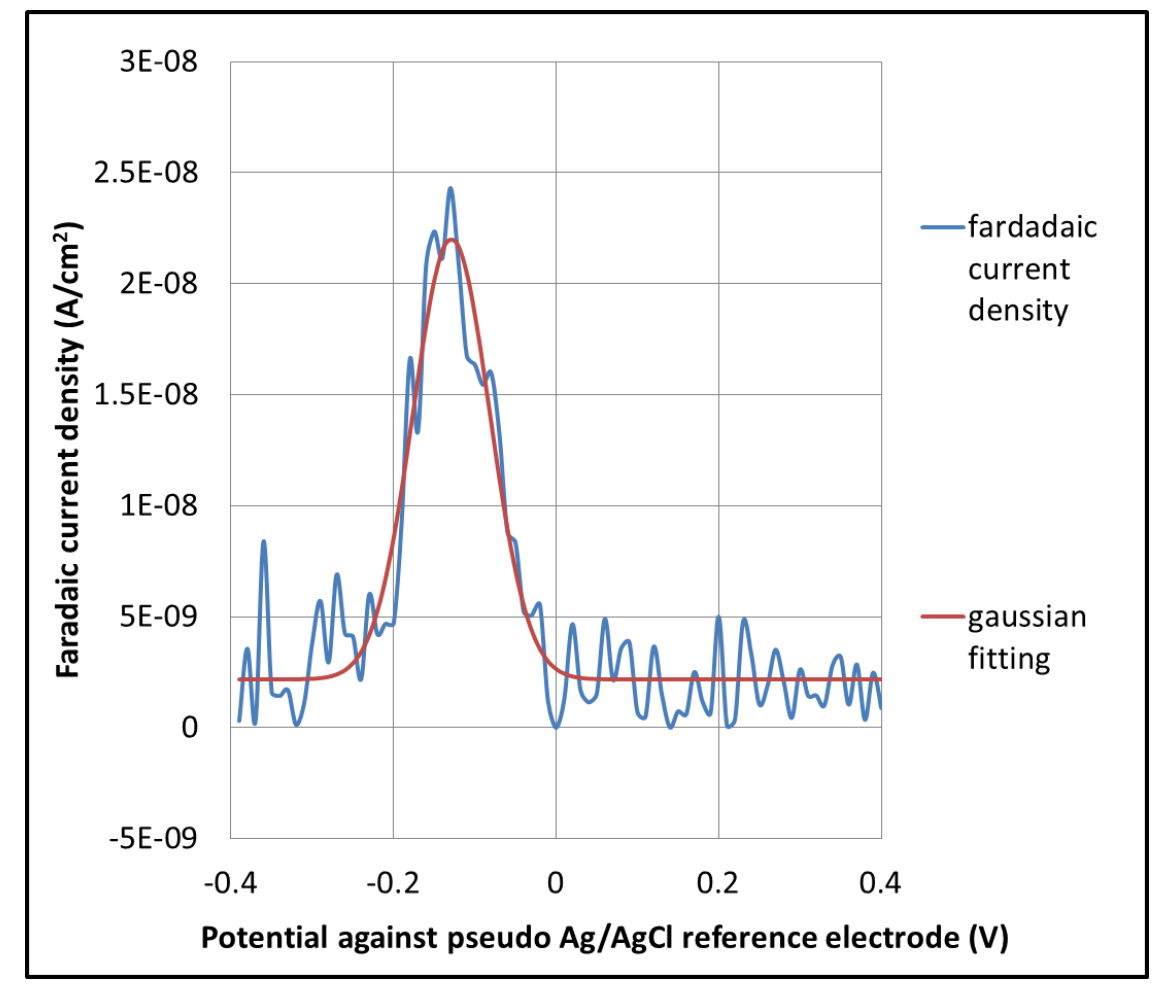

Figure 4-5: Gaussian fitting for one segment of the reconstructed faradaic current dentistry against the potential curve.

The apparent electron transfer number, $n_{a}$, was then calculated with Equation 4-5. A value of $n_{a}=1.1$, which was very close to the theoretical unit for the case when the intermolecular interaction was negligible and it was quite reasonable since the surface coverage was just a small fraction of a full monolayer. 


$$
n_{a}=\frac{90.5 \mathrm{mV}}{\Delta E_{1 / 2}}
$$

The active surface coverage was also calculated based on the information of $i_{p}$ with Equation 4-6, where $R$ is the gas constant and $T$ is the experimental temperature (room temperature).

$$
i_{p}=\frac{n^{2} F^{2}}{4 R T} v \Gamma_{a c t}
$$

The active surface coverage was only $1.2 \times 10^{-12} \mathrm{~mol} / \mathrm{cm}^{2}$, which was about $30 \%$ of the total surface coverage of immobilized cytochrome c. The possible reasons for this phenomenon could be as follows. First, the ITO surface has inactive sites and electrons could not be transferred through those sites to cytochrome c proteins. The optical signal could still detect their presence at originally oxidized state as they are immobilized on the surface. Another possibility is that conformational changes in the cytochrome c molecules during adsorption process are so severe that the electron transfer path is damaged or completely blocked.

\section{Electrical current data}

Electrical current density signals were calculated by using the electrical current data measured by the potentiostat with an effective working electrode area $\left(4.1 \times 1.4 \mathrm{~cm}^{2}\right)$. As shown in Figure $4-6$, no clear faradaic current density peaks could be identified for cytochrome c proteins contained signal. There was no significant difference between the reference and the cytochrome c protein signals. 


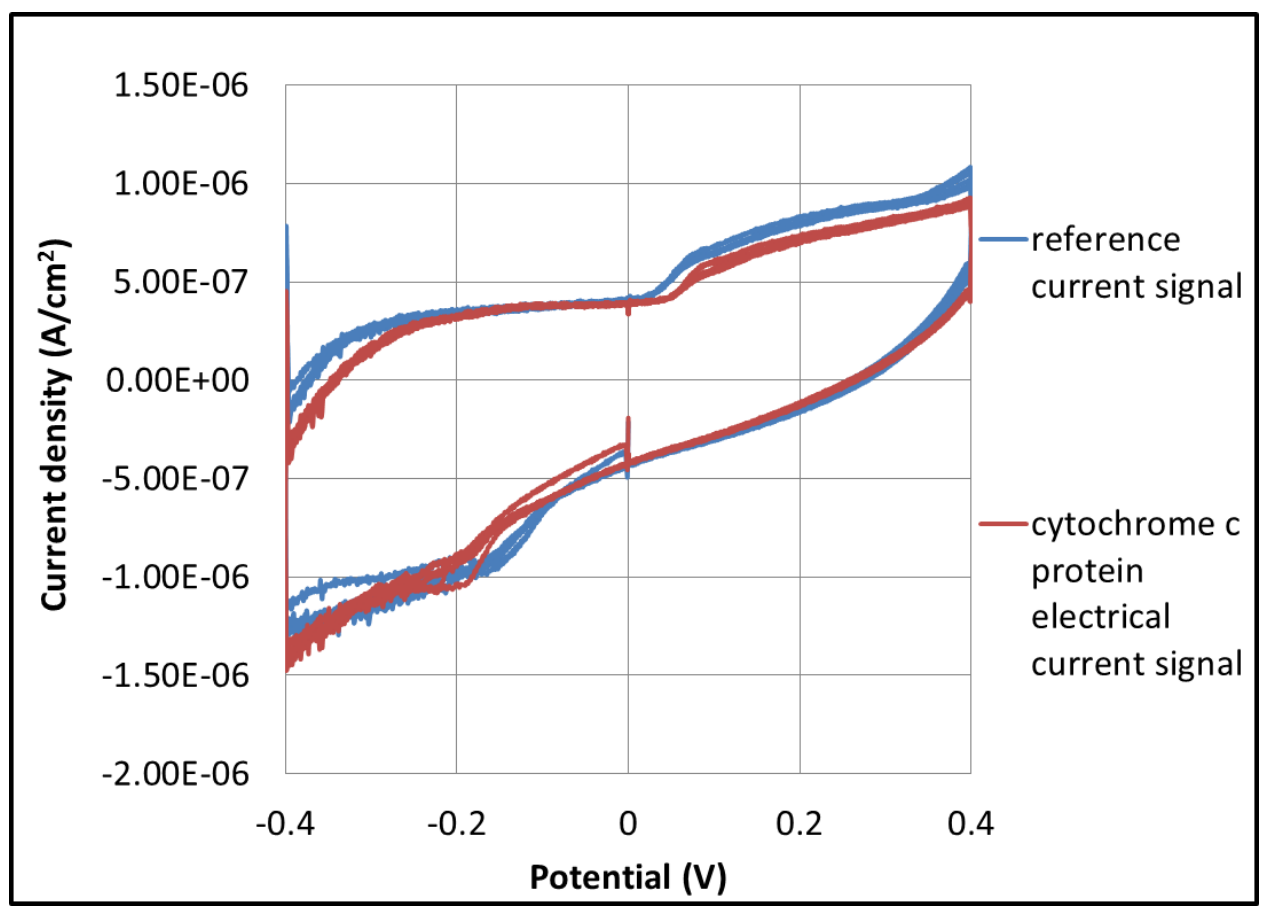

Figure 4-6: Electrical current density calculated by using the potentiostat measured electrical current signal and an effective area of the EA-SM-IOW surface. No difference can be observed between the reference and the protein signals.

From optical signals, the reconstructed faradaic current density of redox species at sub-monolayer (Figure 4-4) is at the scale of $10^{-8} \mathrm{~A} / \mathrm{cm}^{2}$. The total electrical current density (Figure 4-6), mainly the charging current of the double layer capacitance, measured by the potentiostat is at the scale of $10^{-6} \mathrm{~A} / \mathrm{cm}^{2}$. This is a clear demonstration of the superior performance of the EA-SM-IOW to address molecular thin films involved electrochemical redox reactions by avoiding the strong background current signal. 


\section{Conclusions}

Under linear CV potential scans, the redox reaction for a sub-monolayer cytochrome c protein was demonstrated by using an EA-SM-IOW as a working electrode with a wave-guided light for optical interrogation. The faradaic current density was reconstructed from the optical signal at $550 \mathrm{~nm}$ to characterize the formal potential, the potential-width of the electrochemical reaction, and active surface coverage. The total surface coverage was calculated by using an isosbestic wavelength $557 \mathrm{~nm}$. For the cytochrome c proteins adsorbed to the ITO surface of the EA-SM-IOW, only a fraction of the total surface adsorbed species was electrically active under the CV scans. As expected, conventional electrochemical current measurements were unable to detect and quantify faradaic current events as the surface coverage was below its limit of detection.

To obtain kinetics information of the electron transfer processes, an AC potential modulation is needed. In Chapter 5, a new analysis strategy is developed for an optical impedance spectroscopy technique to study electron transfer rates for surface-adsorbates at sub-monolayer level in a simple and accurate manner. 


\title{
CHAPTER 5
}

\section{OPTICAL IMPEDANCE SPECTROSCOPY FOR KINETIC STUDIES OF REDOX REACTIONS}

\author{
Introduction
}

\begin{abstract}
Alternating current $(\mathrm{AC})$ potential modulation is the most used technique to obtain the electron transfer rate for surface immobilized redox species. Only the responding signals (electrical current or optical signal) with the same frequency as the driving potential can be recorded, and the noise level is reduced. Theories and analytical methods have been developed to improve electrochemical impedance spectroscopy (EIS) and optical impedance spectroscopy (OIS) measurements.
\end{abstract}

Theory for redox reactions of surface immobilized species

For the analysis of conventional electrochemical techniques, an equivalent electrical circuit is usually used. For the case of surface-adsorbates presence in the flow cell, one simple representation for the electrochemical is shown in Figure 5-1. The solution resistance is $R_{S}$. The surface immobilized molecular layer is embedded inside of the double layer capacitance, $C_{d}$. The equivalent electrical circuit for the redox reaction of the molecules is in parallel with $C_{d}$. The total potential modulation, 
$E$, is applied across the whole flow cell, while the potential modulation applied on the molecular thin film is $E^{F}$. Under the condition of no diffusion, the equivalent electrical elements for the molecular thin film under redox reaction can be represented by a charge transfer resistance, $R_{c t}$, and a pseudo capacitance, $C_{a}$, in series [198]. The deductions processes of $R_{c t}$ and $C_{a}$ are as follows.

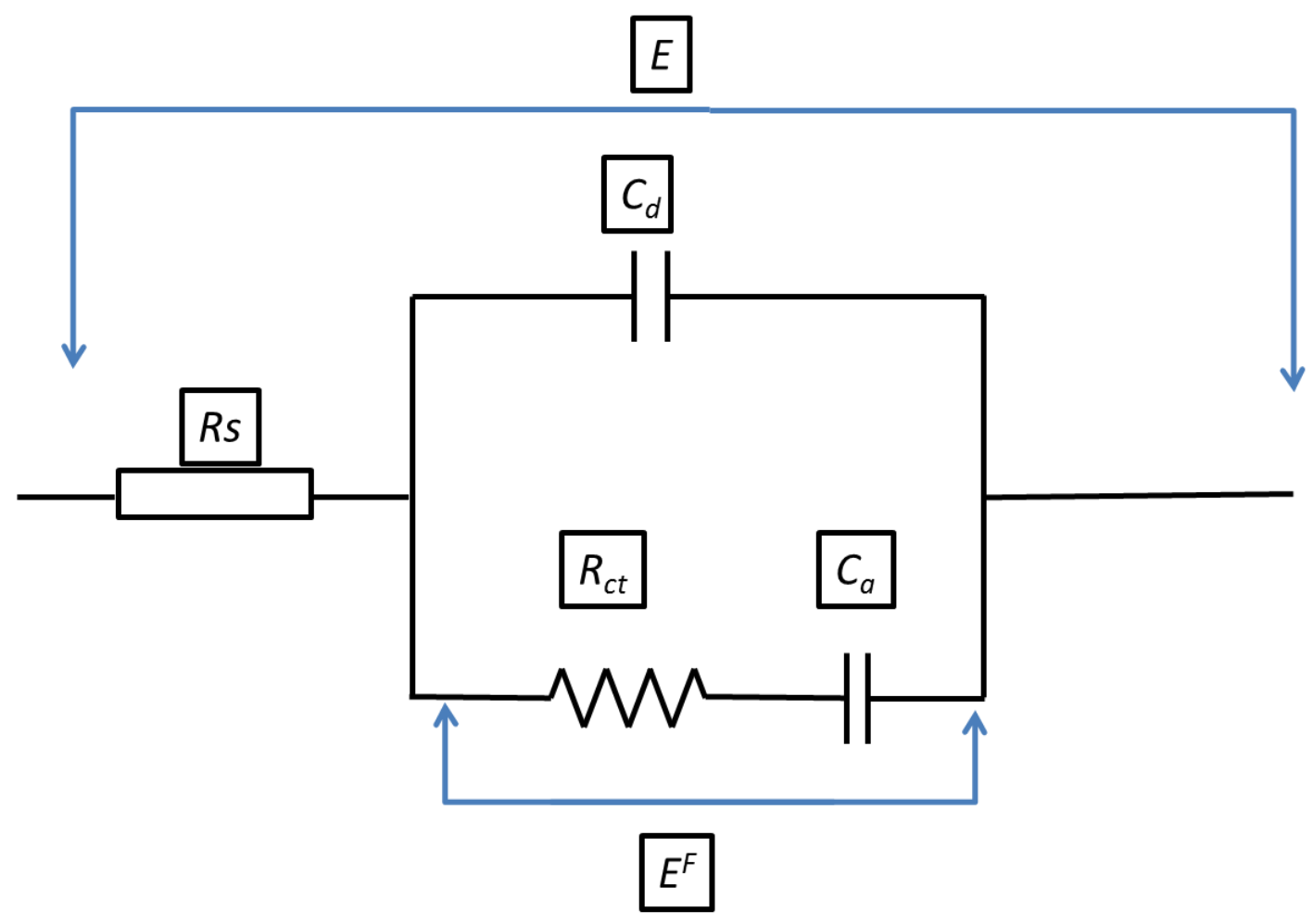

Figure 5-1: An equivalent electrical circuit for an electrochemical flow cell with a molecular thin film immobilized on the working electrode surface. The electrolyte solution has a resistance, $R_{S}$. The equivalent electrical circuit for the redox reaction of the surface-adsorbates is represented by a charge transfer resistance, $R_{c t}$, in series with a pseudo capacitance, $C_{a}$. And this circuit is in parallel with the double layer capacitance, $C_{d}$. 
The applied total potential $E$ to the working electrode of an electrochemical cell determines the values of $I_{F}$ (the faradaic current), $\Gamma_{o x}$ (the surface density of species at oxidized state), and $\Gamma_{\text {tot }}$ (the total density of surface-adsorbed species). The relationship of these parameters can be mathematically described by Equation $5-1$.

$$
E=E\left(I_{F}, \Gamma_{o x}, \Gamma_{t o t}\right)
$$

The total surface coverage is simply the sum of the surface coverage of the reduced and oxidized species.

$$
\Gamma_{t o t}=\Gamma_{o x}+\Gamma_{\text {red }}
$$

By taking the derivative of Equation 5-1 with respect to time $t$, we have:

$$
\frac{d E}{d t}=\left.\frac{d I_{F}}{d t} \frac{\partial E}{\partial I_{F}}\right|_{\Gamma_{o x}, \Gamma_{t o t}}+\left.\frac{d \Gamma_{o x}}{d t} \frac{\partial E}{\partial \Gamma_{o x}}\right|_{I_{F}, \Gamma_{t o t}}+\left.\frac{d \Gamma_{t o t}}{d t} \frac{\partial E}{\partial \Gamma_{t o t}}\right|_{I_{F}, \Gamma_{o x}}
$$

By considering that the redox species are immobilized on the electrode surface with no net desorption or adsorption, $\Gamma_{t o t}$ is a time-constant,

$$
\frac{d \Gamma_{t o t}}{d t}=0=\frac{d \Gamma_{r e d}}{d t}+\frac{d \Gamma_{o x}}{d t}
$$

and Equation 5-2 can be modified to:

$$
\frac{d E}{d t}=\left.\frac{d I_{F}}{d t} \frac{\partial E}{\partial I_{F}}\right|_{\Gamma_{o x}}+\left.\frac{d \Gamma_{o x}}{d t} \frac{\partial E}{\partial \Gamma_{o x}}\right|_{I_{F}}
$$

The faradaic current, $I_{F}$, is directly related to the change of the surface coverage of adsorbed species at reduced(oxidized) state under the potential 
modulation, as given by Equation 5-3, where $A_{d e v}$ is the area of the electrochemical active surface of the working electrode.

$$
I_{F}=-\mathrm{A}_{\mathrm{dev}} n F \frac{d \Gamma_{r e d}}{d t}=\mathrm{A}_{\mathrm{dev}} n F \frac{d \Gamma_{o x}}{d t}
$$

With the expression of the faradaic current in Equation 5-3, Equation 5-2 can be further simplified to:

$$
\frac{d E}{d t}=\left.\frac{d I_{F}}{d t} \frac{\partial E}{\partial I_{F}}\right|_{\Gamma_{o x}}+\left.\frac{I_{F}}{n F} \frac{\partial E}{\partial \Gamma_{o x}}\right|_{I_{F}}
$$

The right hand side of this equation above has two components. The first term can be considered as an equivalent resistance for the charge transfer process, $R_{c t}$, and the second term as pseudo-capacitance, $C_{a}$, associated with the same electrochemical process. Those relations are summarized Equation 5-4.

$$
\begin{gathered}
\left.R_{c t} \equiv \frac{1}{A_{d e v}} \frac{\partial E}{\partial I_{F}}\right|_{\Gamma_{O x}} \\
C_{a} \equiv \frac{n F}{\left.\frac{1}{A_{\text {dev }}} \frac{\partial E}{\partial \Gamma_{o x}}\right|_{I_{F}}}
\end{gathered}
$$

The faradaic current can be expressed in the Butler-Volmer equation [199] as Equation 5-5, where $k_{0}$ is the electron transfer rate between the redox species and the working electrode, and $\alpha$ is the electron transfer coefficient.

$$
I_{F}=n F k_{0}\left[-\Gamma_{o x} \exp \left\{-\frac{\alpha n_{a} F\left(E-E_{0}\right)}{R T}\right\}+\left(\Gamma_{\text {tot }}-\Gamma_{o x}\right) \exp \left\{\frac{(1-\alpha) n_{a} F\left(E-E_{0}\right)}{R T}\right\}\right]
$$

The expression for $R_{c t}$ can be derived by taking the derivative on both sides of Equation 5-5with respect to $I_{F}$ : 


$$
\begin{aligned}
1=n F k_{0}\left[\Gamma_{o x} \frac{\alpha n_{a} F}{R T} \exp \left\{-\frac{\alpha n_{a} F\left(E-E_{0}\right)}{R T}\right\} \frac{\partial E}{\partial I_{F}}+\left(\Gamma_{t o t}\right.\right. \\
\left.\left.-\Gamma_{o x}\right) \frac{(1-\alpha) n_{a} F}{R T} \exp \left\{\frac{(1-\alpha) n_{a} F\left(E-E_{0}\right)}{R T}\right\} \frac{\partial E}{\partial I_{F}}\right]
\end{aligned}
$$

When the magnitude of the over potential, $\left|E-E_{0}\right|$, is much smaller than $R T / n_{a} F$, a linear approximation can be used for the exponential functions in the previous equation. With this linear approximation, a simplified expression is achieved.

$$
\begin{aligned}
& 1 \cong n F k_{0}\left[\Gamma_{o x} \frac{\alpha n_{a} F}{R T} \frac{\partial E}{\partial I_{F}}+\Gamma_{r e d} \frac{(1-\alpha) n_{a} F}{R T} \frac{\partial E}{\partial I_{F}}\right]=n F k_{0} \frac{n_{a} F}{R T} \frac{\partial E}{\partial I_{F}}\left[\alpha \Gamma_{o x}+\right. \\
& \left.(1-\alpha)\left(\Gamma_{t o t}-\Gamma_{o x}\right)\right]
\end{aligned}
$$

For $\alpha \cong 0.5$, we have: $\alpha \Gamma_{o x}+(1-\alpha)\left(\Gamma_{t o t}-\Gamma_{o x}\right) \cong \frac{\Gamma_{t o t}}{2}$, which gives us the expression for $R_{c t}$ as shown in Equation 5-6.

$$
R_{c t} \equiv \frac{1}{A_{d e v}} \frac{\partial E}{\partial I_{F}}=\frac{2 R T}{\Gamma_{t o t} n n_{a} F^{2} k_{0} A_{d e v}}
$$

For the pseudo capacitance, the deduction process is as follows. By taking the derivative on both sides of Equation 5-5 with respect to $\Gamma_{o x}$ under the condition of constant $\Gamma_{\text {tot }}$ and $I_{F}$, we obtain:

$$
\begin{array}{r}
0=-\exp \left\{-\frac{\alpha n_{a} F\left(E-E_{0}\right)}{R T}\right\}+\Gamma_{o x}\left(\frac{\alpha n_{a} F}{R T}\right) \exp \left\{-\frac{\alpha n_{a} F\left(E-E_{0}\right)}{R T}\right\} \frac{\partial E}{\partial \Gamma_{o x}}- \\
\exp \left\{\frac{(1-\alpha) n_{a} F\left(E-E_{0}\right)}{R T}\right\}+\left(\Gamma_{\text {tot }}-\Gamma_{o x}\right) \frac{(1-\alpha) n_{a} F}{R T} \exp \left\{\frac{(1-\alpha) n_{a} F\left(E-E_{0}\right)}{R T}\right\} \frac{\partial E}{\partial \Gamma_{o x}}
\end{array}
$$

By using the linear approximation and $\alpha \cong 0.5$, we then obtain:

$$
\frac{\partial E}{\partial \Gamma_{o x}}=\frac{4 R T}{\Gamma_{t o t} n_{a} F}
$$


The pseudo capacitance, associated with the electrochemical reaction, is then expressed as Equation 5-7.

$$
C_{a}=\frac{\Gamma_{t o t} n n_{a} F^{2} A_{d e v}}{4 R T}
$$

Based on the expressions of $R_{c t}$ and $C_{a}$, the electron transfer rate can be expressed as Equation 5-8 for surface immobilized redox species.

$$
k_{0}=\left(2 R_{c t} C_{a}\right)^{-1}
$$

Conventional electrochemical impedance spectroscopy

In conventional EIS, the solution resistance $R_{S}$ is obtained at a high modulation frequency (so that the double-layer capacitance contribution to impedance is negligible), and the double layer capacitance $C_{d}$ is obtained at a low modulation frequency without redox species present in the flow cell. After AC impedance measurements for a surface-immobilized redox thin film, the values of $R_{c t}$ and $C_{a}$ are adjusted for impedance simulations to match the experimental results. This method is demonstrated in Figure 5-2 [49]. In A, the electrical current in phase and out of phase components at a modulation frequency of $21 \mathrm{~Hz}$ is used to determine the formal potential. In $\mathrm{B}$, the in phase and out of phase components of the impedance at the formal potential $+0.013 \mathrm{~V}$ for the tested redox species are plotted against the modulation frequency. The equivalent electrical circuits for the flow cell with and without the thin layer of redox species presence are shown. With the best fitting between the experimental impedances and simulated impedances, 
the values of $R_{c t}$ and $C_{a}$ are determined. And the electron transfer rate can be calculated with Equation 5-8.
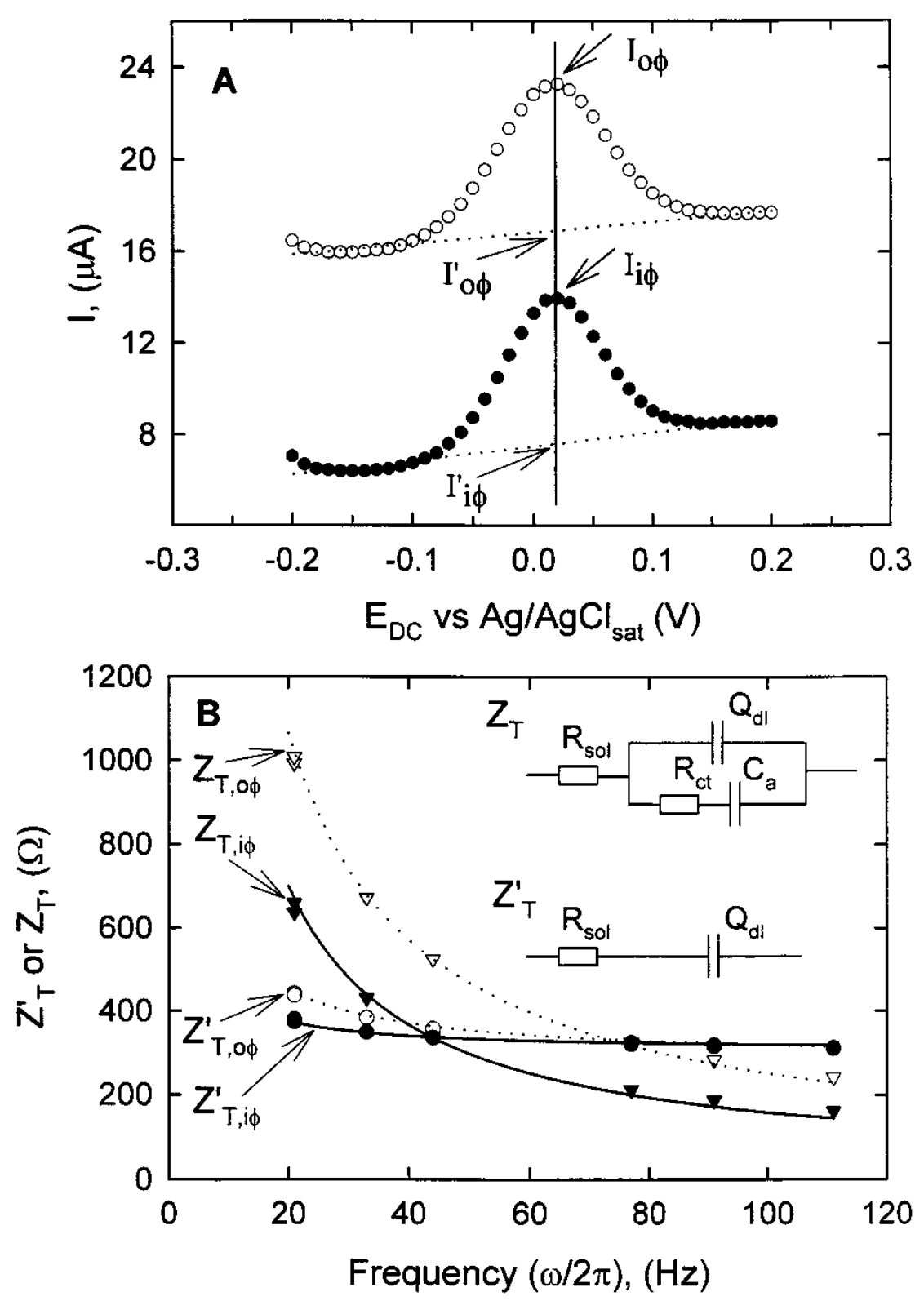

Figure 5-2: Electrochemical impedance measurements and simulation results for flow cell with and without protein presence. In A, both the real and imaginary components of the current are plotted against potential. In B, experimental results 
of the real and imaginary components of impedance spectroscopies for the electrochemical flow cell without and with redox species are plotted against modulation frequency. The simulation results by using the equivalent electrical circuits are plotted to match the experiment results. Resource "Electron Transfer between Surface-Confined Cytochrome c and an N acetylcysteine modified gold electrode. Langmuir, 1998."

For the EIS measurements, the background current signal from the double layer capacitance is strong, and the equivalent electrical circuit is over simplified. For some electrochemical flow cell configurations, other possible sources could contribute to the impedance. And a simulation result by using this simple circuit, cannot match the experiment data.

\section{A Novel Analytical Methodology for Optical Impedance Spectroscopy}

Based on the optimized ultra-thin ITO films from Section 2.3.3, EA-SM-IOW were developed to implement highly sensitive OIS measurements. As the initial examination has demonstrated, the transparent conductive electrode (ITO) does change its optical properties under the potential modulation. A new methodology is described to factor out those optical background changes from the working electrode and retrieve the accurate electrochemical information for redox adsorbates. 
Under an AC impedance potential modulation, as expressed in Equation 3-2 and under the linear approximation for a small amplitude potential modulation, the reference optical signal (here labeled with subscript " 0 ") and the redox adsorbates present optical signal are expressed in a similar format with a DC component, an AC component and a phase delay, as described in Equation 5-9

$$
\begin{aligned}
& I_{0}(t)=I_{d c, 0}\left(E_{d c}\right)+\Delta I_{a c, 0}\left(\omega, E_{d c}\right) \sin \left[\omega t+\theta\left(\omega, E_{d c}\right)\right] \\
& I(t)=I_{d c}\left(E_{d c}\right)+\Delta I_{a c}\left(\omega, E_{d c}\right) \sin \left[\omega t+\theta\left(\omega, E_{d c}\right)\right]
\end{aligned}
$$

\section{Calculation of absorbance under AC modulation}

With its definition, the absorbance for the redox species under the $\mathrm{AC}$ potential modulation can be expressed in Equation 5-10.

$$
A b s\left(E_{d c}, \omega, t\right)=-\log _{10}\left(\frac{I_{d c}\left(E_{d c}\right)+I_{a c}\left(E_{d c}, \omega\right) \sin [\omega t+\theta(\omega)]}{I_{d c, 0}\left(E_{d c}\right)+I_{a c, 0}\left(E_{d c}, \omega\right) \sin \left[\omega t+\theta_{0}(\omega)\right]}\right)
$$

Next, the Equation 5-10 above is manipulated to express the absorbance with a DC and an AC component:

$$
\begin{gathered}
A b s\left(E_{d c}, \omega, t\right)=-\log _{10}\left(I_{d c}\left(E_{d c}\right)+I_{a c}\left(E_{d c}, \omega\right) \sin [\omega t+\theta(\omega)]\right)+ \\
\log _{10}\left(I_{d c, 0}\left(E_{d c}\right)+I_{a c, 0}\left(E_{d c}, \omega\right) \sin \left[\omega t+\theta_{0}(\omega)\right]\right) \\
=\log _{10}\left(I_{d c}\left(E_{d c}\right)\right)-\frac{\ln \left(1+\frac{I_{a c}\left(E_{d c}, \omega\right) \sin [\omega t+\theta(\omega)]}{I_{d c}\left(E_{d c}\right)}\right)}{\ln (10)}+ \\
\log _{10}\left(I_{d c, 0}\left(E_{d c}\right)\right)+\frac{\ln \left(1+\frac{I_{a c, 0}\left(E_{d c}, \omega\right) \sin \left[\omega t+\theta_{0}(\omega)\right]}{I_{d c, 0}\left(E_{d c}\right)}\right)}{\ln (10)}
\end{gathered}
$$


Then the $\mathrm{DC}$ and $\mathrm{AC}$ components of the absorbance can be separated and expressed in Equation 5-11 and 5-12 separately. The DC component of absorbance is only related to the DC component of the potential modulation, and is related the amount of the active surface coverage and total surface coverage of the redox adsorbates, the same as expressed in Equation 4-1.

$$
\begin{aligned}
& A b s_{d c}\left(E_{d c}\right)=-\log _{10}\left(\frac{I_{d c}\left(E_{d c}\right)}{I_{d c, 0}\left(E_{d c}\right)}\right) \\
& A b s_{a c}\left(E_{d c}, t\right)=-\frac{\ln \left(1+\frac{I_{a c}\left(E_{d c}, \omega\right) \sin [\omega t+\theta(\omega)]}{I_{d c}\left(E_{d c}\right)}\right)}{\ln (10)}+\frac{\ln \left(1+\frac{I_{a c, 0}\left(E_{d c}, \omega\right) \sin \left[\omega t+\theta_{0}(\omega)\right]}{I_{d c, 0}\left(E_{d c}\right)}\right)}{\ln (10)}
\end{aligned}
$$

Since $\frac{I_{a c, 0}\left(E_{d c}, \omega\right)}{I_{d c, 0}\left(E_{d c}\right)}$ or $\frac{I_{a c}\left(E_{d c}, \omega\right)}{I_{d c}\left(E_{d c}\right)}$ is a small number compared to 1 (because of the small AC amplitude of the applied potential modulation, and experimentally confirmed in section 5.3.3), Equation 5-12 can be simplified as in Equation 5-13 with a linear approximation.

$$
A b s_{a c}\left(E_{d c}, t\right) \cong-\frac{I_{a c}\left(E_{d c}, \omega\right) \sin [\omega t+\theta(\omega)]}{\ln (10) I_{d c}\left(E_{d c}\right)}+\frac{I_{a c, 0}\left(E_{d c}, \omega\right) \sin \left[\omega t+\theta_{0}(\omega)\right]}{\ln (10) I_{d c, 0}\left(E_{d c}\right)}
$$

With Equation 5-13, the amplitude of the in phase and out of phase components of the $\mathrm{AC}$ absorbance can be expressed in Equation 5-14 and Equation 5-15 separately:

$$
\begin{aligned}
& \Delta A b s_{a c}^{\prime}=\left(-\frac{I_{a c}\left(E_{d c}, \omega\right) \cos [\theta(\omega)]}{\ln (10) I_{d c}\left(E_{d c}\right)}+\frac{I_{a c, 0}\left(E_{d c}, \omega\right) \cos \left[\theta_{0}(\omega)\right]}{\ln (10) I_{d c, 0}\left(E_{d c}\right)}\right) \\
& \Delta A b s_{a c}^{\prime \prime}=\left(-\frac{I_{a c}\left(E_{d c}, \omega\right) \sin [\theta(\omega)]}{\ln (10) I_{d c}\left(E_{d c}\right)}+\frac{I_{a c, 0}\left(E_{d c}, \omega\right) \sin \left[\theta_{0}(\omega)\right]}{\ln (10) I_{d c, 0}\left(E_{d c}\right)}\right)
\end{aligned}
$$


The AC component of the absorbance signal is determined by the amount of the surface immobilized redox species driven by the AC potential amplitude, with Equation 5-16.

$$
A b s_{a c}\left(E_{d c}, \omega\right)=\sqrt{\Delta A b s^{\prime}{ }_{a c}^{2}+\Delta A b s^{\prime \prime}{ }_{a c}^{2}}=S \Gamma_{a c}\left(E_{d c}, \omega\right) \Delta \epsilon
$$

\section{Reconstruction of faradaic current density}

As previously shown in Equation 4-3 shows, by taking the derivative of the absorbance with respect to time the faradaic current density can be reconstructed. The imaginary component of the faradaic current density is reconstructed by taking the derivative of the real component of the $\mathrm{AC}$ absorbance, as in Equation 5-17 The real component of the faradaic current density is reconstructed by taking the derivative of the imaginary component of the AC absorbance, as in Equation 5-18 The amplitude of the faradaic current density is expressed as Equation 5-19.

$$
\begin{aligned}
& \Delta i_{a c, \mathrm{~F}}^{\prime}\left(E_{d c}, \omega\right)=-\omega \frac{n F}{s \Delta \epsilon} \Delta A_{a c}^{\prime \prime} \\
& \Delta i_{a c, F}^{\prime \prime}\left(E_{d c}, \omega\right)=\omega \frac{n F}{s \Delta \epsilon} \Delta A_{a c}^{\prime} \\
& i_{a c, F}=\sqrt{\Delta i_{a c, F}^{\prime 2}+\Delta i_{a c, F}^{\prime \prime 2}}
\end{aligned}
$$

\section{Determination of electron transfer rate}

A generalized equivalent electrical circuit, as shown in Figure 5-3, is modeled here for the spectroelectrochemical flow cell. The impedance elements in the whole flow cell are generically called $Z_{1}$ and $Z_{2}$, which include the buffer solution 
resistance, the ITO impedance, the resistance of the electrical connections and other possible sources.

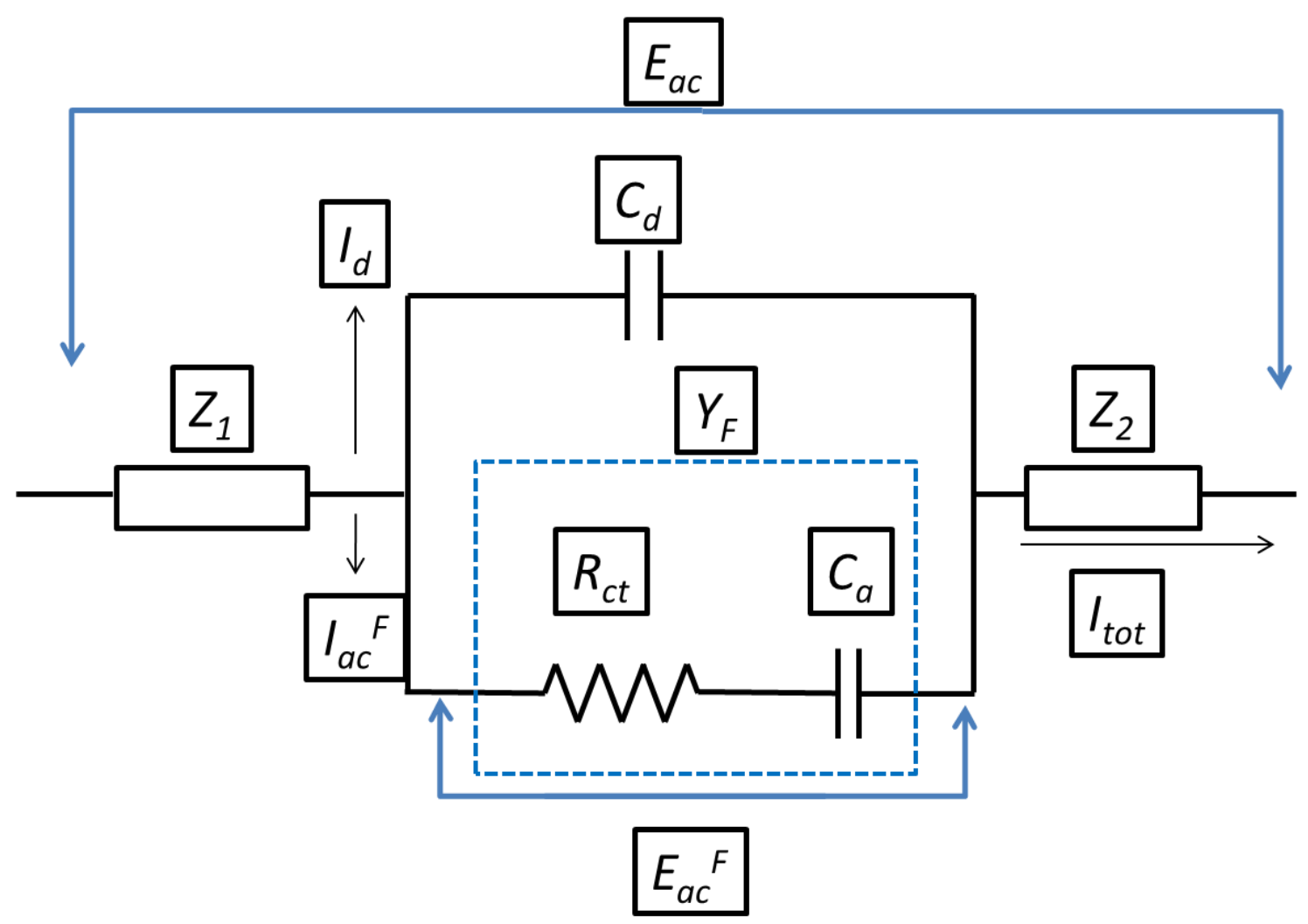

Figure 5-3: Generalized equivalent electrical circuit for the spectroelectrochemical flow cell. Impedance of the electrochemical flow cell is generalized with $Z_{1}$ and $Z_{2}$, which includes the resistance of the electrolyte solution and other possible impedance sources.

The total AC potential modulation applied across the entire circuit in the electrochemical cell is called $E_{a c}$, and the potential modulation across the molecular 
thin film under redox reaction is $E_{a c}^{F}$. The equivalent electrical circuit for the redox reaction of the surface-adsorbates can be represented by a faradaic admittance, $Y_{F}$. Instead of calculating $E_{a c}^{F}$ from specific information on every electrical component present in the circuit, a different methodology is described below to obtain the faradaic admittance related to the redox reaction in the molecular thin film.

The relationship between the total current, $I_{t o t}$, the total impedance of the whole flow cell, $Z_{t o t}$, and the applied potential modulation for the entire flow cell $E_{a c}$ is as shown in Equation 5-20:

$$
I_{t o t}=\frac{E_{a c}}{Z_{t o t}}
$$

The relationship between the faradaic current, $I_{a c}^{F}$, the faradaic admittance, $Y^{F}$ and the applied potential modulation for the surface immobilized redox film, $E_{a c}^{F}$ is described by Equation 5-21.

$$
E_{a c}^{F}=\frac{I_{a c}^{F}}{Y^{F}}
$$

The same potential modulation, $E_{a c}^{F}$, that applied to the molecular redox adsorbate is also applied to the double layer capacitance. The relationship of the charging current of the double layer capacitance, $I_{d}$, the double layer capacitance, $C_{d}$, and the applied potential modulation, $E_{a c}^{F}$, is given by Equation 5-22.

$$
E_{a c}^{F}=\frac{I_{d}}{j \omega C_{d}}=\frac{I_{a c}^{F}}{Y^{F}}
$$

From the experimental results and considerations in Chapter 4, it is known that the faradaic current from a sub-monolayer of redox molecules under potential 
modulation, $I_{a c}^{F}$, is a small fraction of the charging current associated with the double layer capacitance, so the total current in the electrochemical cell can be written as :

$$
\mathrm{I}_{\text {tot }}=\mathrm{I}_{\mathrm{d}}+\mathrm{I}_{\mathrm{ac}}^{\mathrm{F}} \cong \mathrm{I}_{\mathrm{d}}
$$

Then the faradaic admittance can be calculated by substituting $I_{\text {tot }}$ from Equation 5-22 and $I_{d}$ from Equation 5-22 into Equation 5-23.

$$
Y^{F}=j \frac{Z_{t o t} I_{a c}^{F} \omega C_{d}}{E_{a c}}
$$

For the AC impedance measurements, the potentiostat measures the total electrical impedance across the flow cell with the real $Z_{\text {tot }}^{\prime}$ and imaginary $Z_{t o t}^{\prime \prime}$ components at each modulation frequency. As previously described, the faradaic current density, $i_{a c}^{F}$, is optically determined with a reference set by the potential modulation across the cell, $E_{a c}$. With all these known parameters, the faradaic admittance can be calculated as in Equation 5-25. In Equation 5-26 and Equation 527, the real and imaginary components of the faradaic admittance are expressed separately by expanding Equation 5-25. With the experiment data of the optical and the electrical impedance spectroscopies, the faradaic admittance real and imaginary components can be calculated.

$$
\begin{aligned}
& Y^{F}=j \frac{\omega C_{d}}{E_{a c}}\left(I_{a c}^{\prime F}+j I_{a c}^{\prime \prime F}\right)\left(Z_{t o t}^{\prime}+j Z_{t o t}^{\prime \prime}\right) \\
& Y^{\prime F}=-\frac{\omega C_{d}}{E_{a c}}\left(I_{a c}^{\prime F} Z_{t o t}^{\prime \prime}+I_{a c}^{\prime \prime F} Z_{t o t}^{\prime}\right) \\
& Y^{\prime \prime F}=\frac{\omega C_{d}}{E_{a c}}\left(I_{a c}^{\prime F} Z_{t o t}^{\prime}-I_{a c}^{\prime \prime F} Z_{t o t}^{\prime \prime}\right)
\end{aligned}
$$


Since the equivalent electrical circuit for the redox reaction of a molecular adsorbate is represented by a charge transfer resistance, $R_{c t}$, in series with a pseudo capacitance $C_{a}$, then the faradaic admittance can be expressed theoretically as in Equation 5-28, and the $\mathrm{R}_{\mathrm{ct}}$ and $\mathrm{C}_{\mathrm{a}}$ can be expressed by Equation 5-29 and Equation 5-30, respectively.

$$
\begin{aligned}
& \frac{1}{Y^{F}}=R_{c t}+\frac{1}{j \omega C_{a}} \\
& R_{c t}=\frac{Y^{F}}{\left|Y^{F}\right|^{2}} \\
& C_{a}=\frac{\left|Y^{F}\right|^{2}}{\omega \prime^{\prime} \prime^{F}}
\end{aligned}
$$

By substituting the expression of $\mathrm{R}_{\mathrm{ct}}$ and $\mathrm{C}_{\mathrm{a}}$ into Equation 5-8, the electron transfer rate constant can be expressed as Equation 5-31.

$$
k_{0}=\frac{1}{2 R_{c t} C_{a}}=\frac{1}{2} \frac{Y \prime^{\prime} F}{Y \prime^{F}} \omega=-\frac{1}{2}\left(\frac{I_{a c}^{\prime F} Z_{t o t}^{\prime}-I_{c c}^{\prime \prime F} Z_{t o t}^{\prime \prime}}{I_{a c}^{\prime F} Z_{t o t}^{\prime \prime}+I_{a c}^{\prime \prime F} Z_{t o t}^{\prime}}\right) \omega
$$

A linear fit of $y=\omega Y^{\prime \prime F}(\omega)$ against $x=2 Y^{\prime F}(\omega)$ calculated over several modulated angular frequencies, $\omega$, can be used, and the slope of this curve will provide the electron transfer rate, $k_{0}$.

In summary, when an $\mathrm{AC}$ potential modulation is performed to drive an electrochemical redox process, the combination of the data from the opticallyretrieved faradaic current, $I_{a c}^{F}(\omega)$ and the data from the electrical impedance across the electrochemical cell, $Z_{\text {tot }}(\omega)$, provides a straightforward approach to determine the electron transfer rate of the surface-adsorbed redox species at sub-monolayer level. 
The generalized equivalent circuit and the analysis for retrieving the reaction rate provide several advantages. First, there is no need to model and determine each component present in the equivalent electrical electrochemical cell. Second, the absolute values of the applied potential modulation, the double layer capacitance, the detection sensitivity, and the difference of molar absorptivity of the redox species are not needed for the determination of the electron transfer rate. OIS with the new methodology described above is tested for the election transfer kinetic of a sub-monolayer of the redox active cytochrome c proteins to show its applicability.

\section{Results from the New Optical Impedance Spectroscopy Analysis}

\section{Experimental procedures}

The experiment set-up with the stationary coupling system was used, the same as Figure 3-10. The probing wavelength $550 \mathrm{~nm}$ was selected. For the AC impedance potential modulations, amplitude of $15 \mathrm{mV}$ and various potential $\mathrm{DC}$ biases (from $-0.2 \mathrm{~V}$ to $0.08 \mathrm{~V}$ ) were applied. The frequencies were from $1 \mathrm{~Hz}$ to $20 \mathrm{~Hz}$ (not angular frequency).

The reference optical signals and the electrical impedance signals were measured first for each potential DC bias with phosphate buffer inside the flow cell. Then approximately $2 \mathrm{ml}$ of $100 \mathrm{nM}$ bulk concentration of cytochrome c solution was injected into the flow cell. An adsorption time of 30 minutes was used to stabilize the adsorption of the cytochrome c proteins on the ITO surface. Then the same optical impedance measurements were carried out. In Figure 5-4, the raw 
optical responses for the protein contained system under AC impedance potential modulations are plotted. The oscillation frequency decreases from left to right.

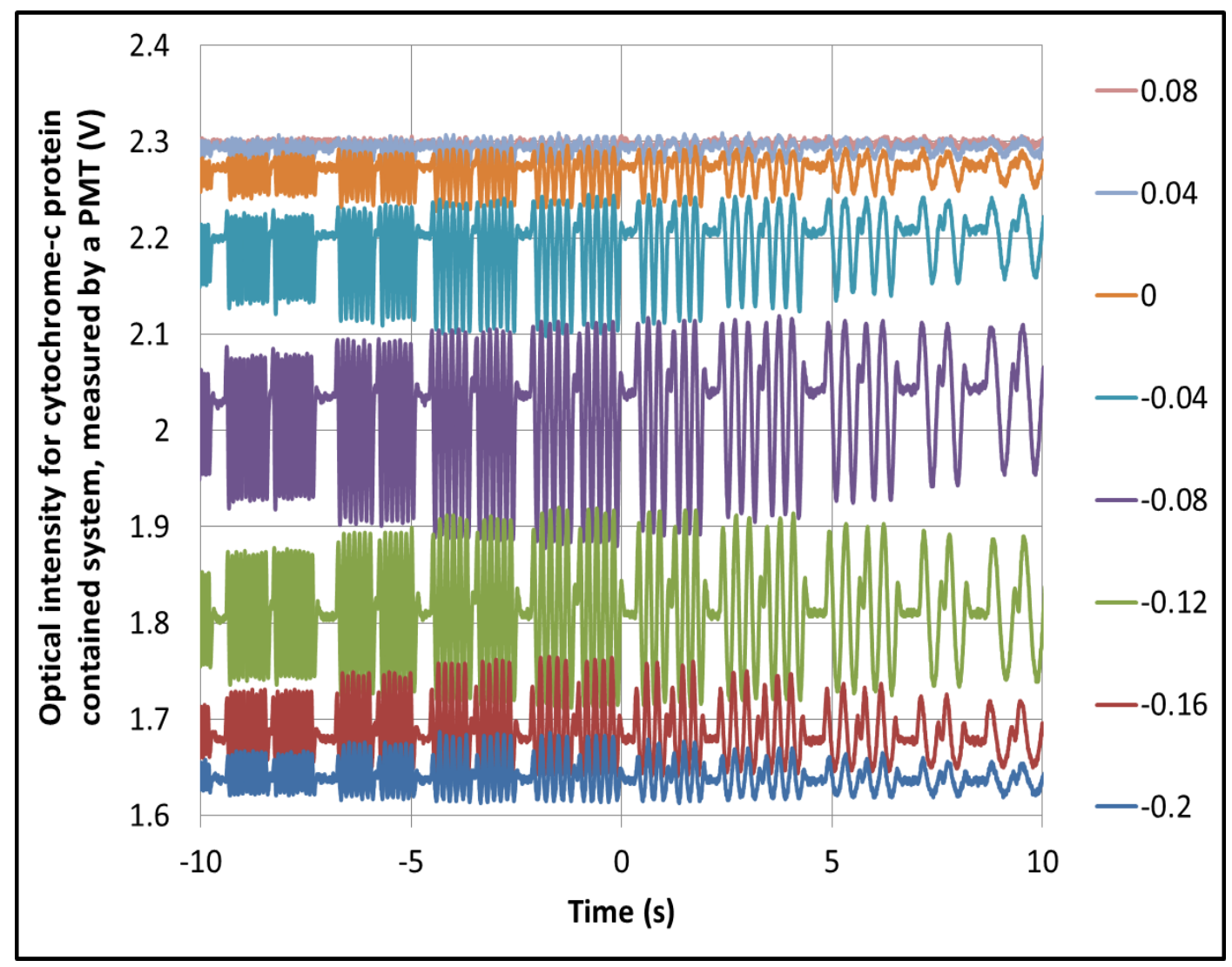

Figure 5-4: Optical response at $550 \mathrm{~nm}$ from cytochrome c protein contained system under AC impedance potential modulations for various potential DC biases.

First, the AC amplitude of the optical response was examined. By qualitative inspection of these curves, the maximum optical AC amplitudes were observed at $0.08 \mathrm{~V}$ and $-0.12 \mathrm{~V}$ DC biases. The formal potential should be close to these two potential DC biases. When the potential DC bias was changed into the positive or 
negative direction, $\mathrm{AC}$ amplitudes of the optical data were smaller. At $+0.08 \mathrm{~V}$ potential DC bias, no more optical AC oscillation could be observed. Most of the cytochrome c proteins were oxidized. Second, the DC amplitudes of the optical responds were examined. The optical DC amplitude stays at a constant through the potential oscillation for each potential DC bias. The separation of the optical DC values was at the maximum between $-0.8 \mathrm{~V}$ and $-0.12 \mathrm{~V}$. The separation became smaller when the potential DC biases increased or decreased. Third, the asymmetry of these traces was examined. Only at - $0.12 \mathrm{~V}$ DC bias, the optical AC oscillation was symmetric. At the more positive potential DC biases, the optical oscillation had higher amplitude toward the formal potential direction, and vice versa for the more negative potential DC biases.

For more quantitative results, the new methodology described in section 5.2 was used. Sinusoidal wave fitting processes were applied to obtain the values of DC, $\mathrm{AC}$ and phase delay of the optical responses.

\section{Analysis of the DC optical response}

As expected, at one specific potential DC bias, the DC component of the optical response was frequency independent. Figure 5-5 shows the average values of the optical DC components, $I_{d c}\left(E_{d c}\right)$, over all frequencies. The blue ones represent the reference optical signal and the red ones represent the signal with surface adsorbed cytochrome c proteins. The standard deviations are shown as error bars. 


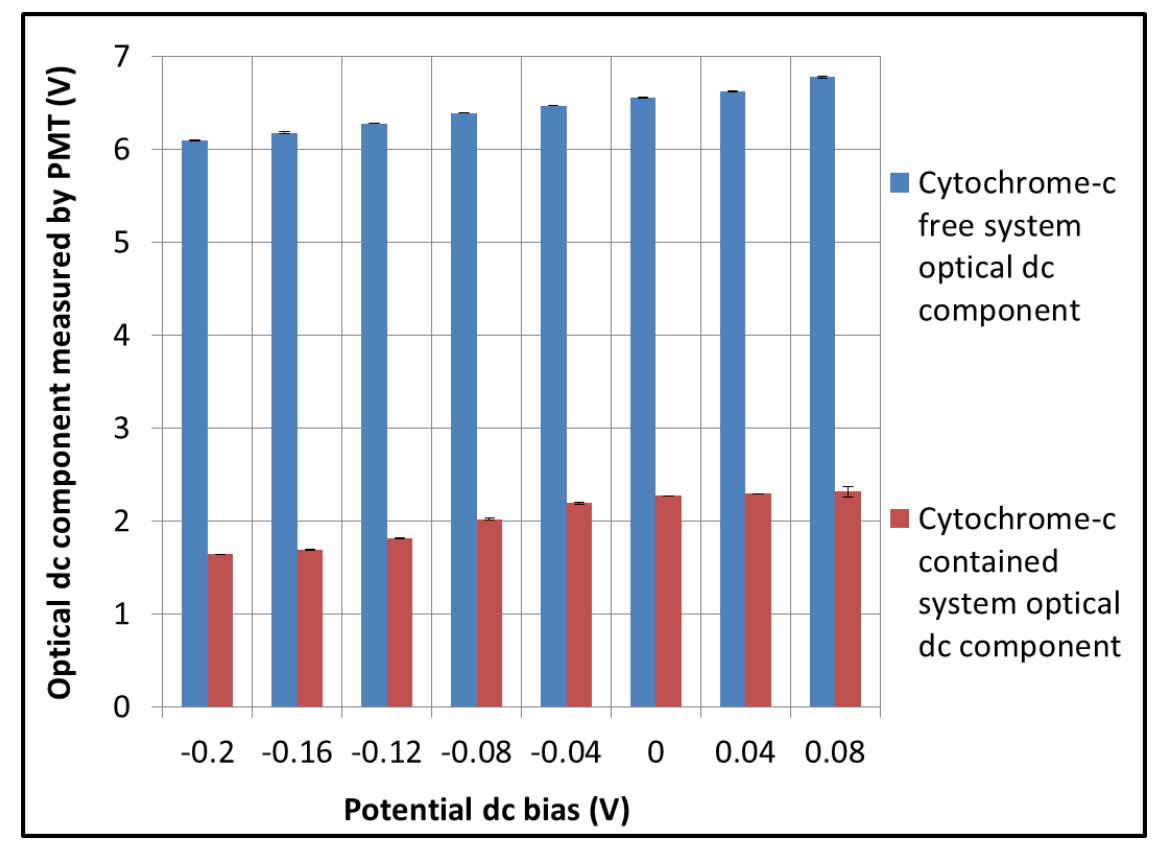

Figure 5-5: DC component of the optical out-coupled intensity at each DC potential bias with average over the frequencies for optical baseline and cytochrome c protein contained optical signals.

With Equation 5-11, the absorbance DC components were calculated, as shown in Figure 5-6 (a). And the relative faradaic current intensity profile was reconstructed by taking the derivative of the DC component of absorbance with respect to the potential DC bias, as shown in Figure 5-6 (b). A Gaussian fit was used to determine the formal potential $(-0.938 \pm 0.001) \mathrm{V}$ and the full width at half maximum (FWHM) $(0.100 \pm 0.004) \mathrm{V}$. 


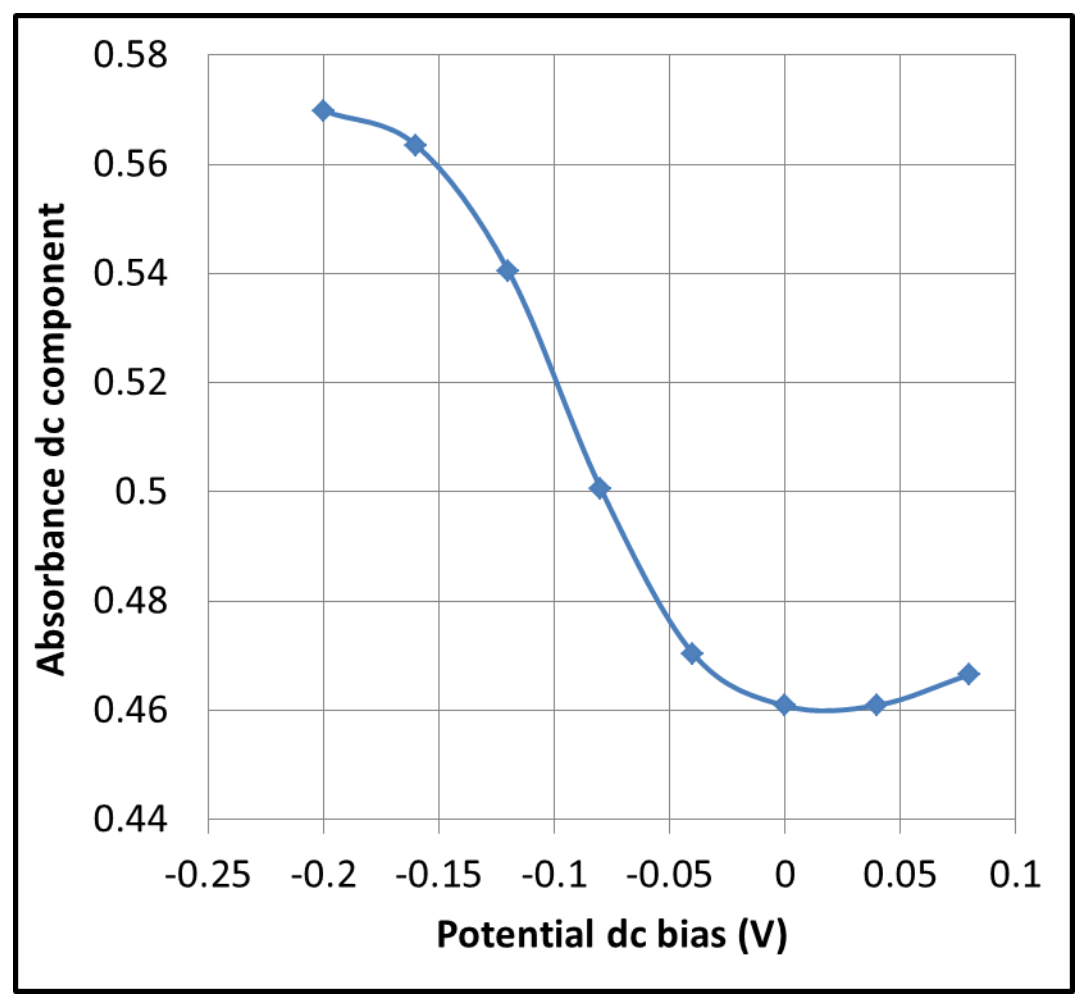

(a)

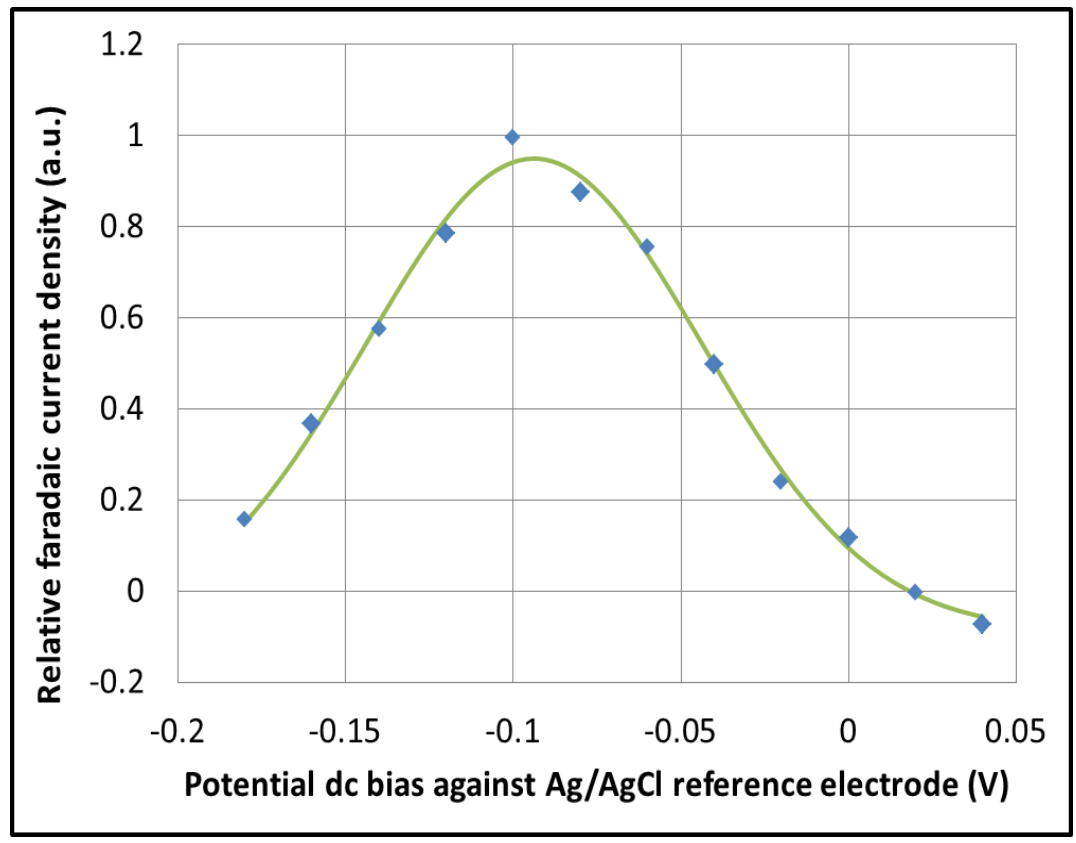

(b)

Figure 5-6: (a) DC component of absorbance against potential DC bias. (b) Reconstructed relative faradaic current density profile with a Gaussian fit. 
The real and imaginary terms for the reference signal Equation 5-14 $\left(\frac{I c, 0\left(E_{d c}, \omega\right) \cos \left[\theta_{0}(\omega)\right]}{\ln (10) I_{d c, 0}\left(E_{d c}\right)} / \frac{I_{a c, 0}\left(E_{d c}, \omega\right) \sin \left[\theta_{0}(\omega)\right]}{\ln (10) I_{d c, 0}\left(E_{d c}\right)}\right)$ and for the cytochrome c proteins optical signal from Equation 5-15 $\left(\frac{I_{a c}\left(E_{d c}, \omega\right) \cos [\theta(\omega)]}{\ln (10) I_{d c}\left(E_{d c}\right)} / \frac{I_{a c}\left(E_{d c}, \omega\right) \sin [\theta(\omega)]}{\ln (10) I_{d c}\left(E_{d c}\right)}\right)$ were calculated. These parameters were plotted in a complex plane (imaginary against real component) to describe the $\mathrm{AC}$ optical absorbance. In Figure 5-7, the results from a few potential DC biases are plotted.

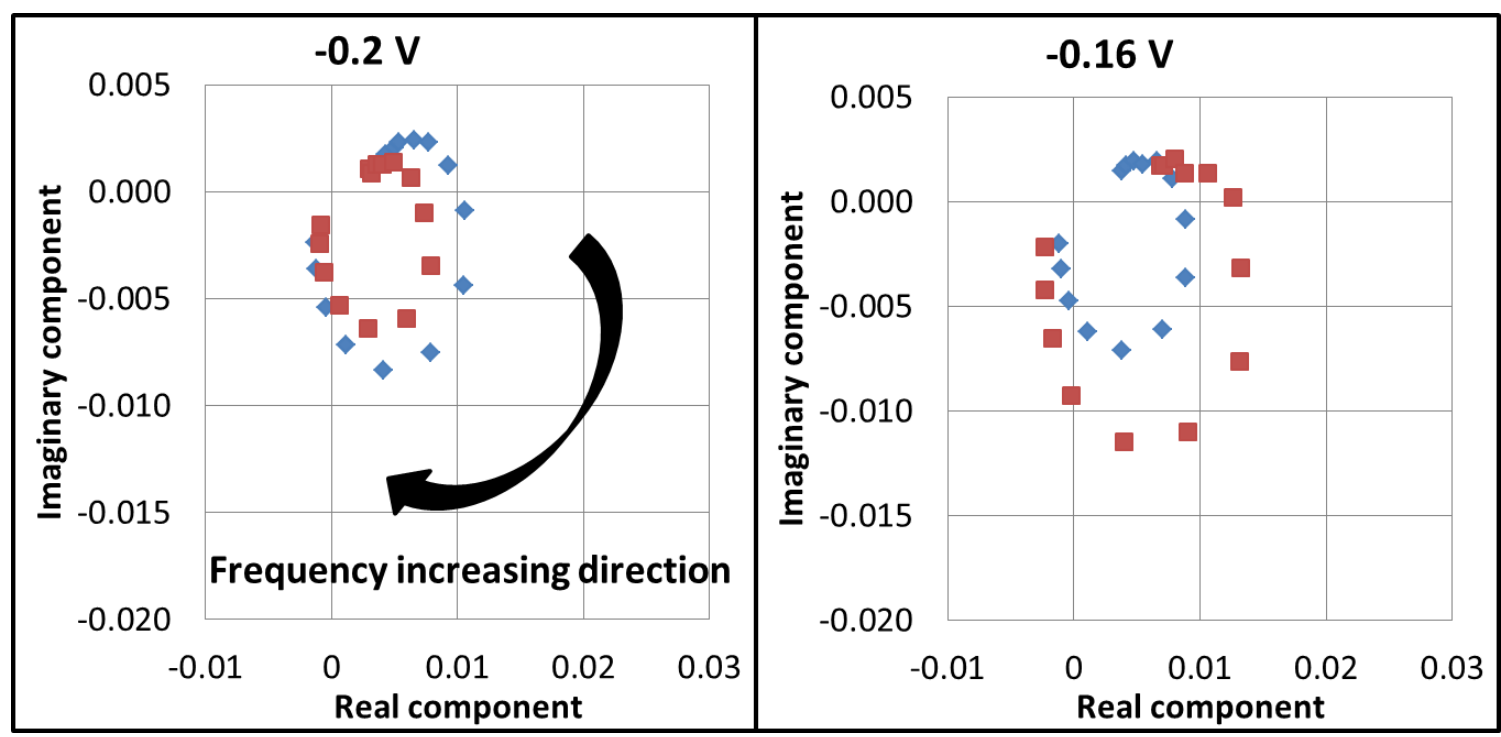



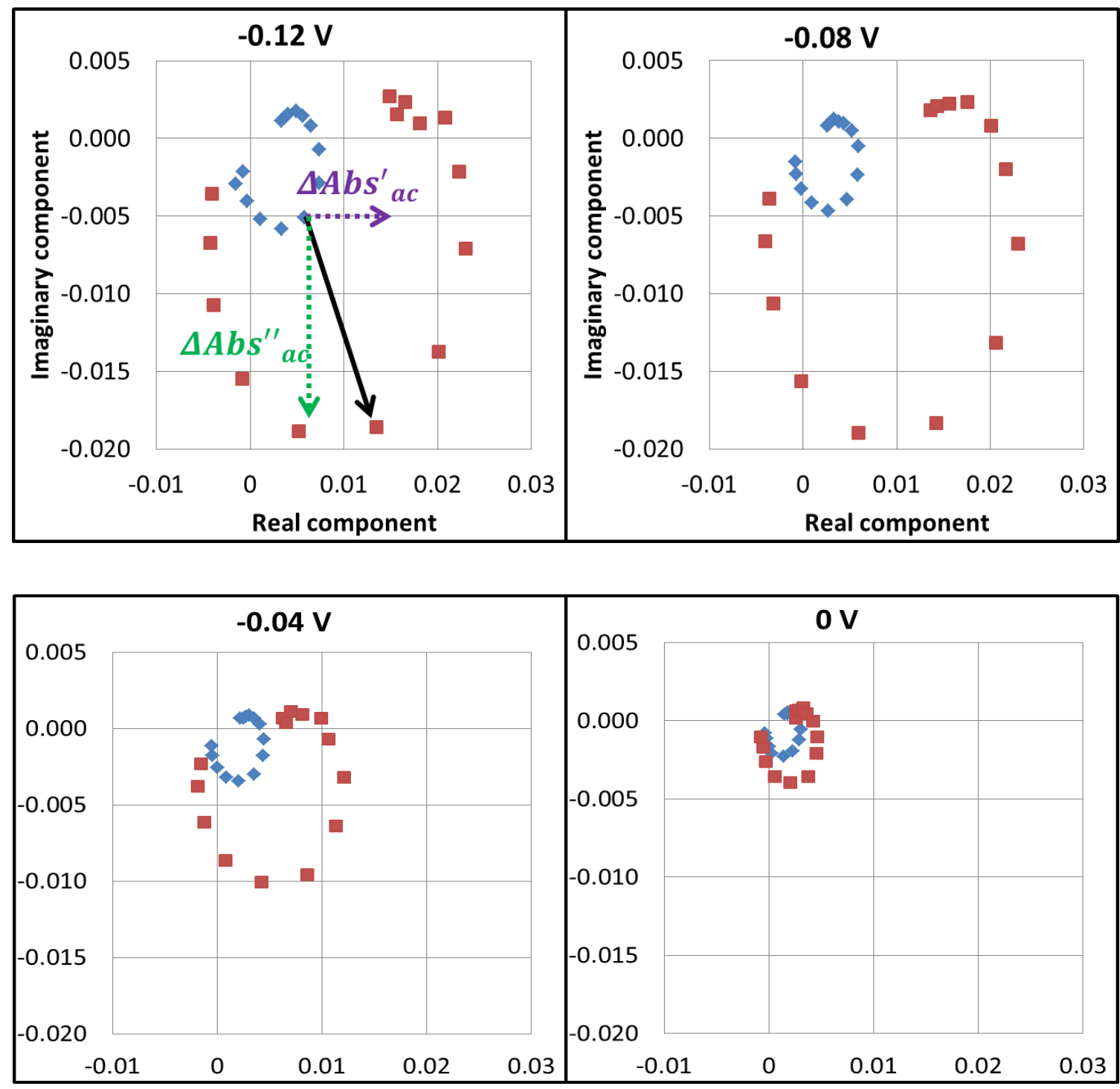

Figure 5-7: Complex planes comparison between the optical reference signal and the proteins signal. The blue diamonds are for the reference signal. The red squares are for cytochrome c proteins signal. All 6 graphs are plotted with the same scale to show differences. The oscillating frequency increases in the clockwise direction, as shown in the plot of the $-0.2 \mathrm{~V}$ potential DC bias. The AC component of the absorbance is represented by a black arrow the drawn from optical baseline to the 
proteins signal with the same frequency. The real component and imaginary component of the $\mathrm{AC}$ absorbance are represented by a purple and a green arrow.

The modulation frequency increases in the clockwise direction, as shown in the plot of $-0.2 \mathrm{~V}$ potential $\mathrm{DC}$ bias. The $\mathrm{AC}$ absorbance amplitude is proportional to the distance in the complex plane between the points corresponding to the protein and reference signals with the same frequency, as shown as in the plot of $-0.12 \mathrm{~V}$ potential DC bias. The black arrow represents the AC component of absorbance, the purple arrow presents the real component, and the green arrow represents the imaginary component of the $\mathrm{AC}$ component of the absorbance signal. At - $0.2 \mathrm{~V}$ and 0 $\mathrm{V}$ potential DC biases, the optical data from the reference and cytochrome c proteins are very close. This means that cytochrome c proteins are mainly reduced or oxidized due to the applied potential DC bias. At $-0.12 \mathrm{~V}$ and $-0.08 \mathrm{~V}$ potential DC biases, the separations between the reference and the protein signals are large. And this matches the optical DC component analysis results that the formal potential should be in this region, i.e., $-0.12 \mathrm{~V}$ and $-0.08 \mathrm{~V}$.

Based on the molar absorptivity difference between the two redox states of the cytochrome c protein and the sensitivity of the EA-SM-IOW at $550 \mathrm{~nm}$, the AC component of the active surface coverage was calculated for each potential DC bias with Equation 5-16. The results are shown in Figure 5-8 in the angular frequency domain for each potential DC bias with the left side y-axis. The AC absorbance curves are shown with the right side y-axis at the same time. 


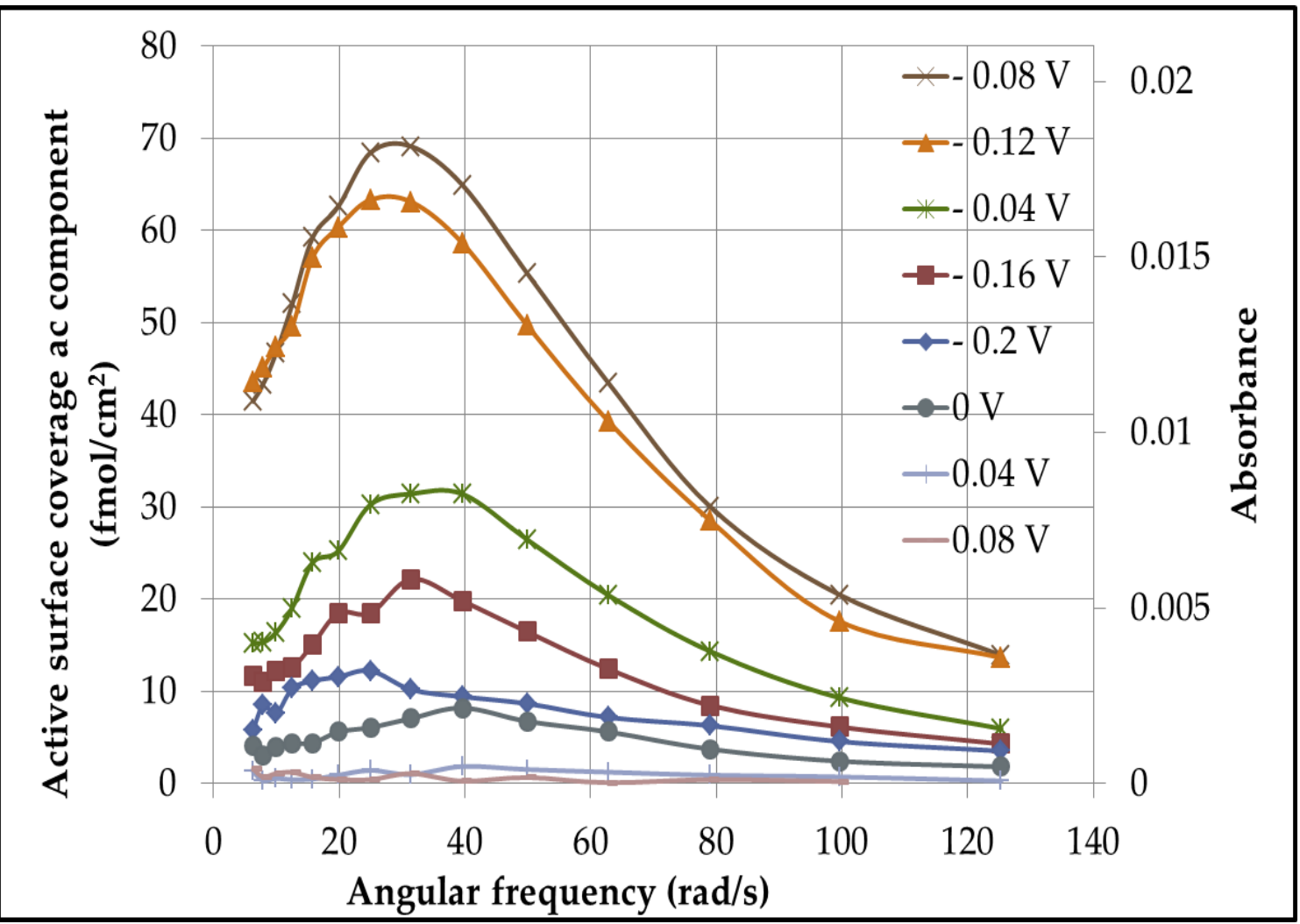

Figure 5-8: With the left side y-axis, the active surface coverage of cytochrome c proteins under $15 \mathrm{mV}$ AC amplitude of impedance potential modulations for each potential DC bias is plotted against angular modulation frequency. With the right side $\mathrm{y}$-axis, the AC absorbance results are plotted.

At $-0.08 \mathrm{~V}$ potential DC bias, a maximum of $7 \times 10^{-14} \mathrm{~mol} / \mathrm{cm}^{2}$ active surface coverage of cytochrome $c$ was detected with the EA-SM-IOW device. When the potential DC bias was away from the formal potential, the active surface coverage decreased. At - $0.16 \mathrm{~V}$ and - $0.04 \mathrm{~V}$ potential DC biases, even smaller active amounts of proteins were detected without any profile distortion. At the extreme positive or negative potential DC biases, the active surface coverage was low. At $+0.04 \mathrm{~V}$ and + 
$0.08 \mathrm{~V}$, no more active surface coverage could be detected. Different amplitudes (5 $\mathrm{mV}, 10 \mathrm{mV}$, and $15 \mathrm{mV}$ ) of the $\mathrm{AC}$ potential modulation were tested and the results confirmed that the $15 \mathrm{mV}$ was comfortably inside the linear response region (Appendix 8).

With the calculated AC absorbance, the real and imaginary components of the faradaic current densities were calculated Equation 5-18 and Equation 5-19. In Figure 5-9, the amplitude of the faradaic current amplitude against the angular frequencies is plotted for various potential DC bias.

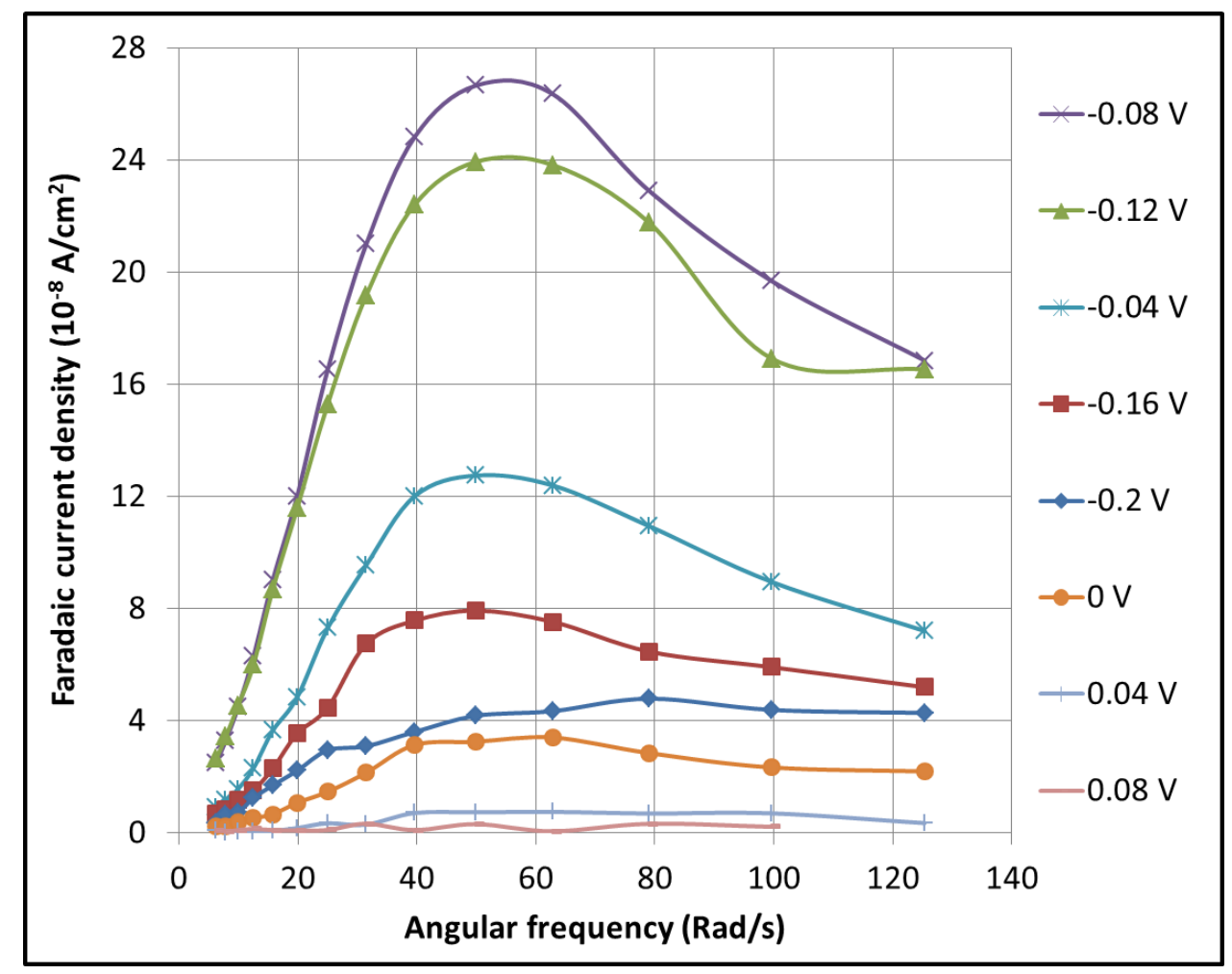

Figure 5-9: Reconstructed faradaic current density for each potential DC bias is plotted against the angular frequency. 
The faradaic current densities at $50 \mathrm{rad} / \mathrm{s}$ angular frequency were plotted against the potential DC biases, as shown in Figure 5-10. A Gaussian fit was used. According to the fitting, the formal potential was $(-0.942 \pm 0.002) \mathrm{V}$ and FWHM was $(0.083 \pm 0.005) \mathrm{V}$.

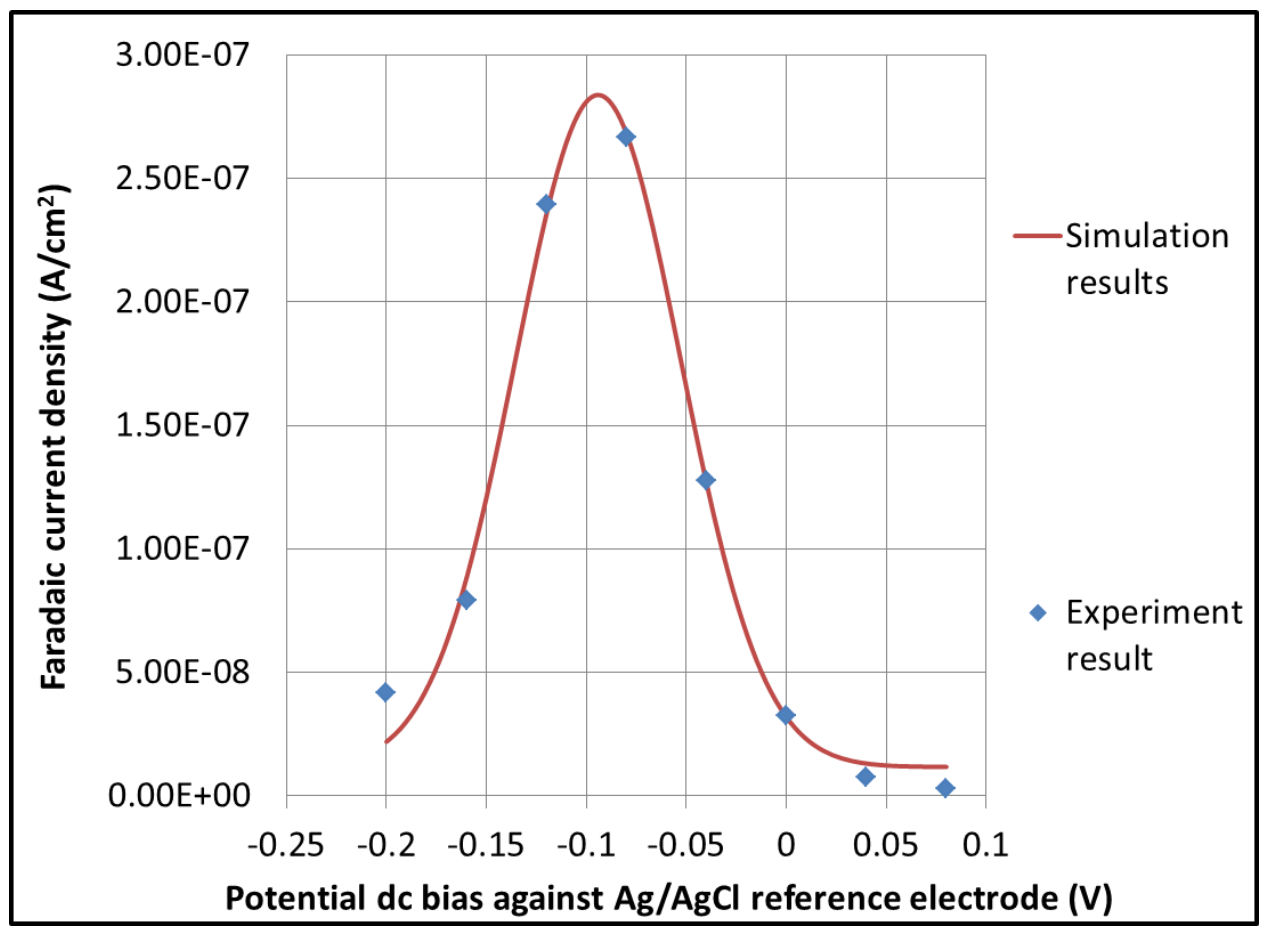

Figure 5-10: Reconstructed faradaic current density against the potential by selecting faradaic current value at $50 \mathrm{rad} / \mathrm{s}$ angular frequency as the blue dots. Gaussian fit is used as in the red solid line.

The formal potential and the FWHM of the faradaic current profiles under the CV scan and the AC impedance potential modulation are compared in Table 5-1. The 
electron transfer process between the surface immobilized cytochrome c proteins and the ITO surface is shown to be quite reversible.

Table 5-1: Comparison of faradaic current profile.

\begin{tabular}{|c|c|c|c|}
\hline $\begin{array}{c}\text { Potential } \\
\text { modulation }\end{array}$ & Formal potential (V) & $\Delta E_{1 / 2}(\mathrm{~V})$ & $n_{a}$ \\
\hline CV & $(-0.131 \pm 0.003) \mathrm{V}$ & $(-0.08 \pm 0.02) \mathrm{V}$ & 1.13 \\
\hline DC of AC impedance & $(0.938 \pm 0.001) \mathrm{V}$ & $(0.100 \pm 0.004) \mathrm{V}$ & 0.96 \\
\hline AC of AC impedance & $(-0.942 \pm 0.002) \mathrm{V}$ & $(0.083 \pm 0.005) \mathrm{V}$ & 0.96 \\
\hline
\end{tabular}

Experimental determination of the electron transfer rate

With Equation 5-31, the electron transfer rate was determined by using the value of the real and the imaginary components of the faradaic admittance. The results from potential DC bias $-0.08 \mathrm{~V}$ and $-0.12 \mathrm{~V}$ were examined, as shown in Figure 5-11 (a) and (b), respectively. From the fitting results, the electron transfer rate (slope) was $28 \mathrm{~s}^{-1}$ for $-0.08 \mathrm{~V}$ and $25 \mathrm{~s}^{-1}$ for $-0.12 \mathrm{~V}$ potential DC bias. 


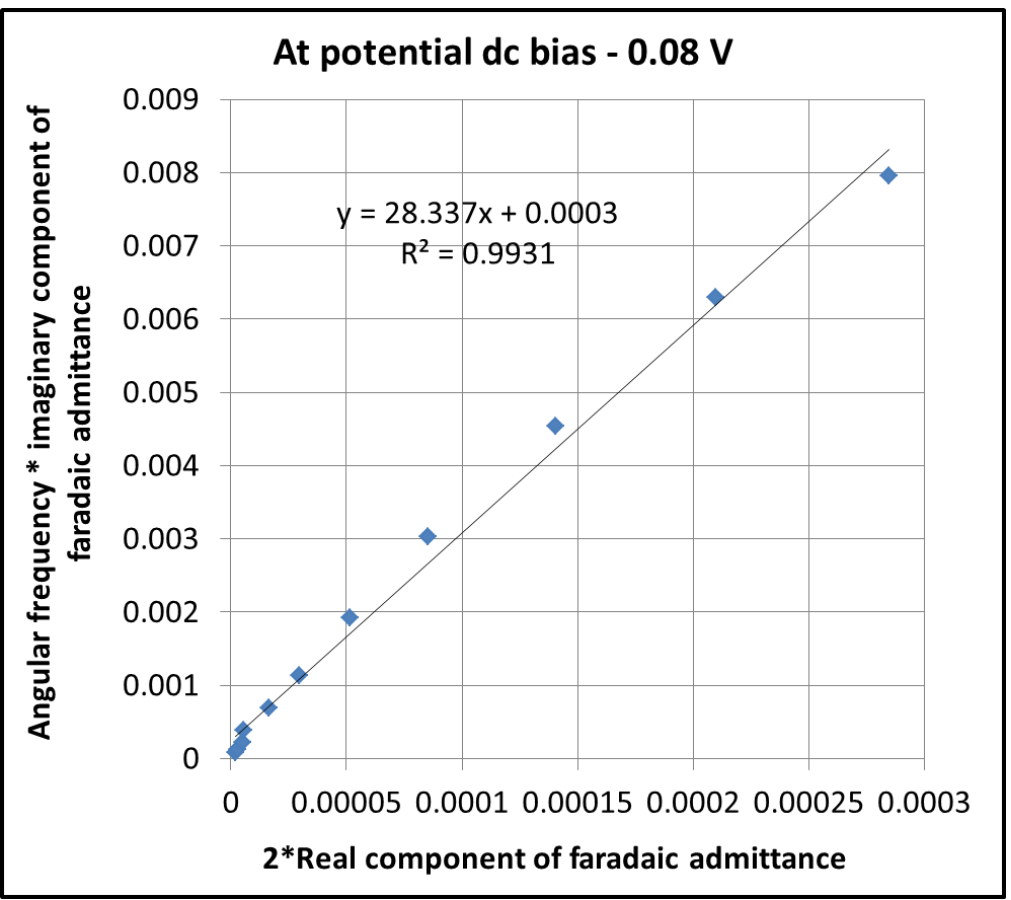

(a)

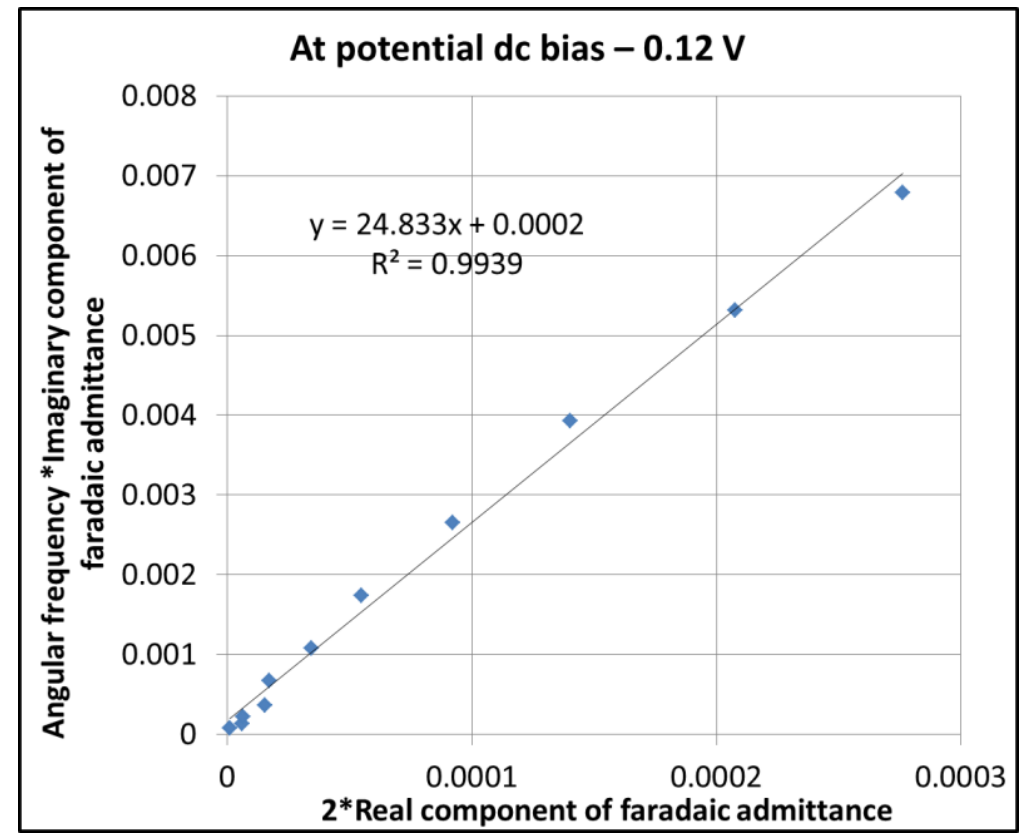

(b)

Figure 5-11: Electron transfer rate calculation for potential DC bias - $0.08 \mathrm{~V}$ (a) and $0.12 \mathrm{~V}$ (b). Linear fit is used, and the slope represents the electron transfer rate. 
With Equation 5-29 and Equation 5-30, the charge transfer resistance $R_{c t}$ and the pseudo capacitance $C_{a}$ were calculated and used to simulate the faradaic admittance. The experiment and the simulation results of faradaic admittance in complex planes from - $0.08 \mathrm{~V}$ and $-0.12 \mathrm{~V}$ DC biases are plotted, as shown in Figure 5-12. Matches are observed for both potential DC biases. The model of an equivalent circuit representation with a charge transfer resistance in series with a pseudo capacitance for the redox reaction from the surface immobilized cytochrome c proteins is justified.

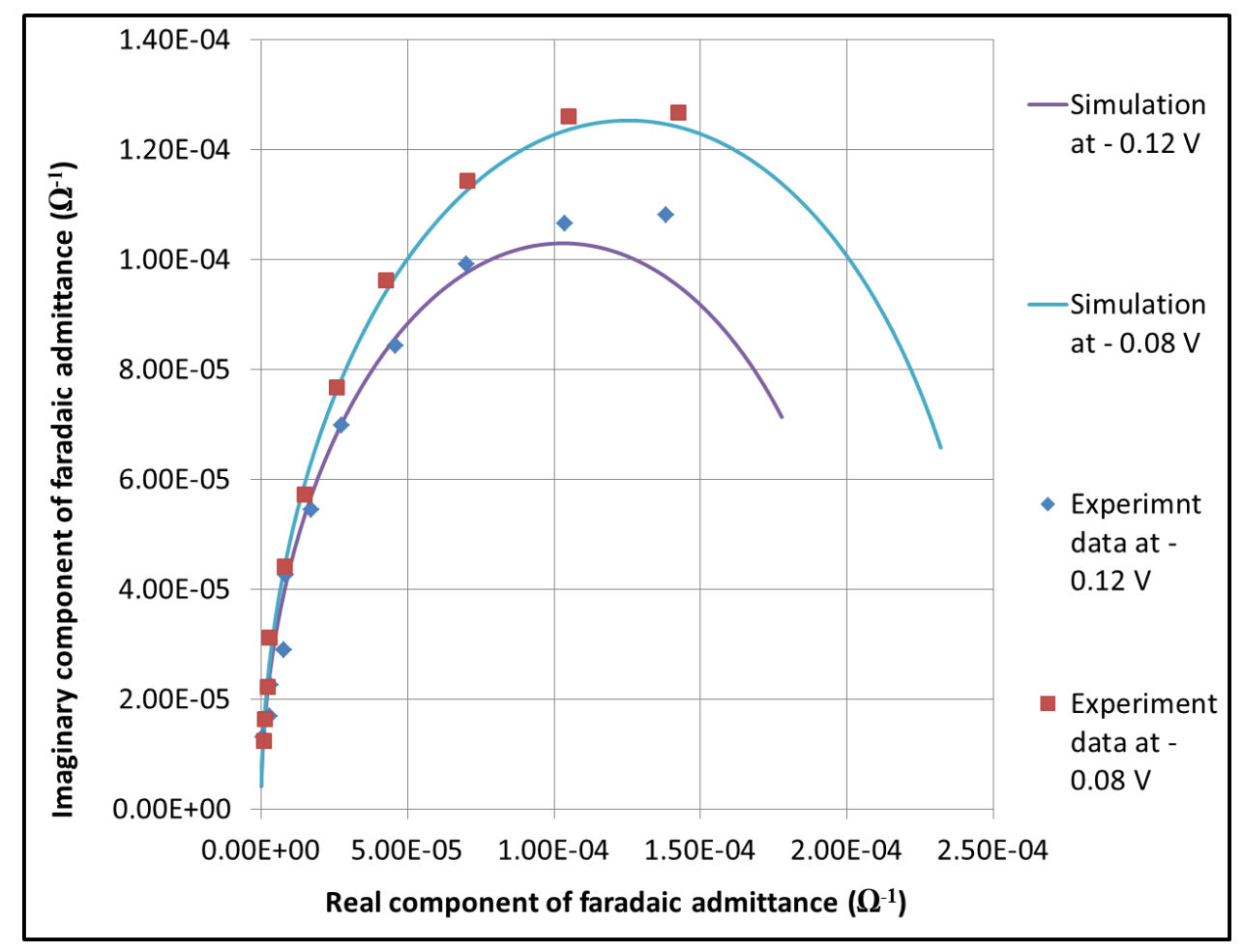

Figure 5-12: Complex planes of the experimental and simulation results of faradaic admittance at $-0.8 \mathrm{~V}$ and $-0.12 \mathrm{~V}$ potential DC biases. Match is observed. 
Finally, the electrical data measured by the conventional EIS technique was examined. For the same sample that was described above by the optical approach, the pure electrical data from the potentiostat were displayed in Figure 5-13 for the real part of the impedance (a), the imaginary part of the impedance (b) and the phase delay (c) at potential DC bias of $-0.08 \mathrm{~V}$.

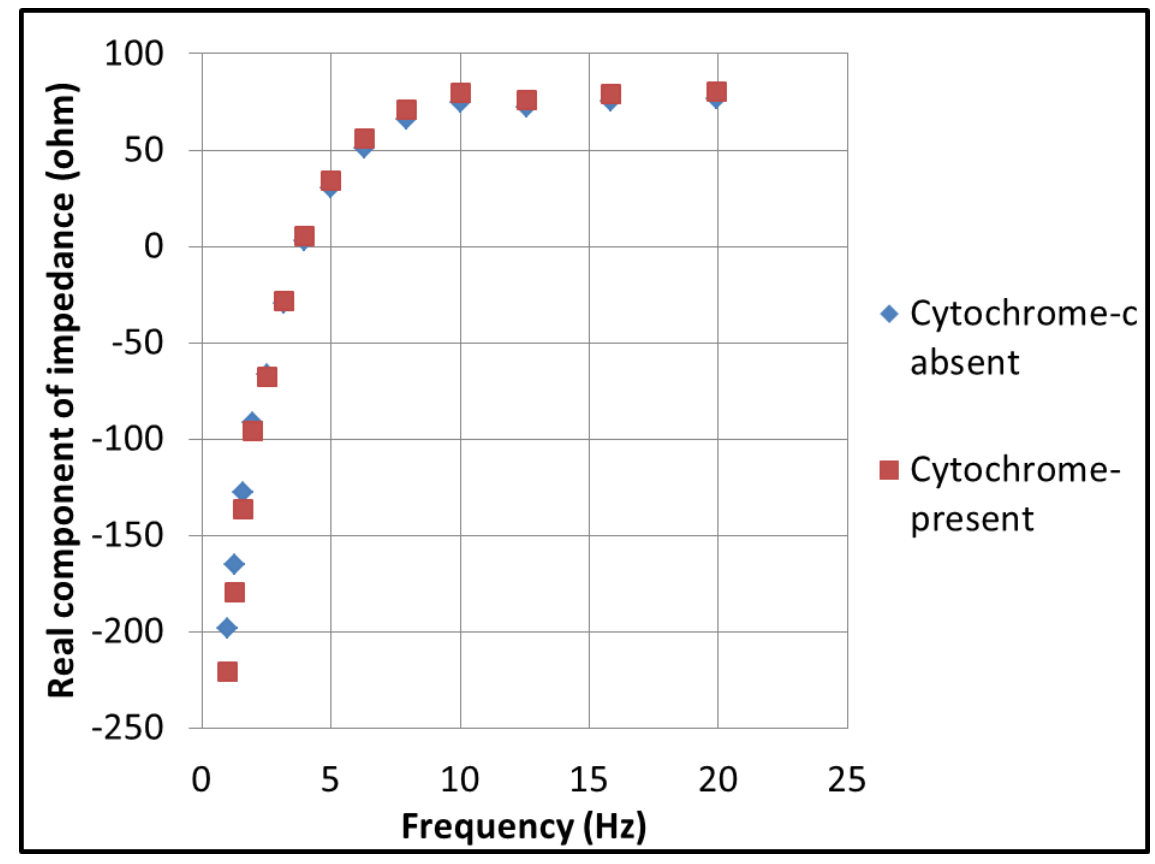

(a) 


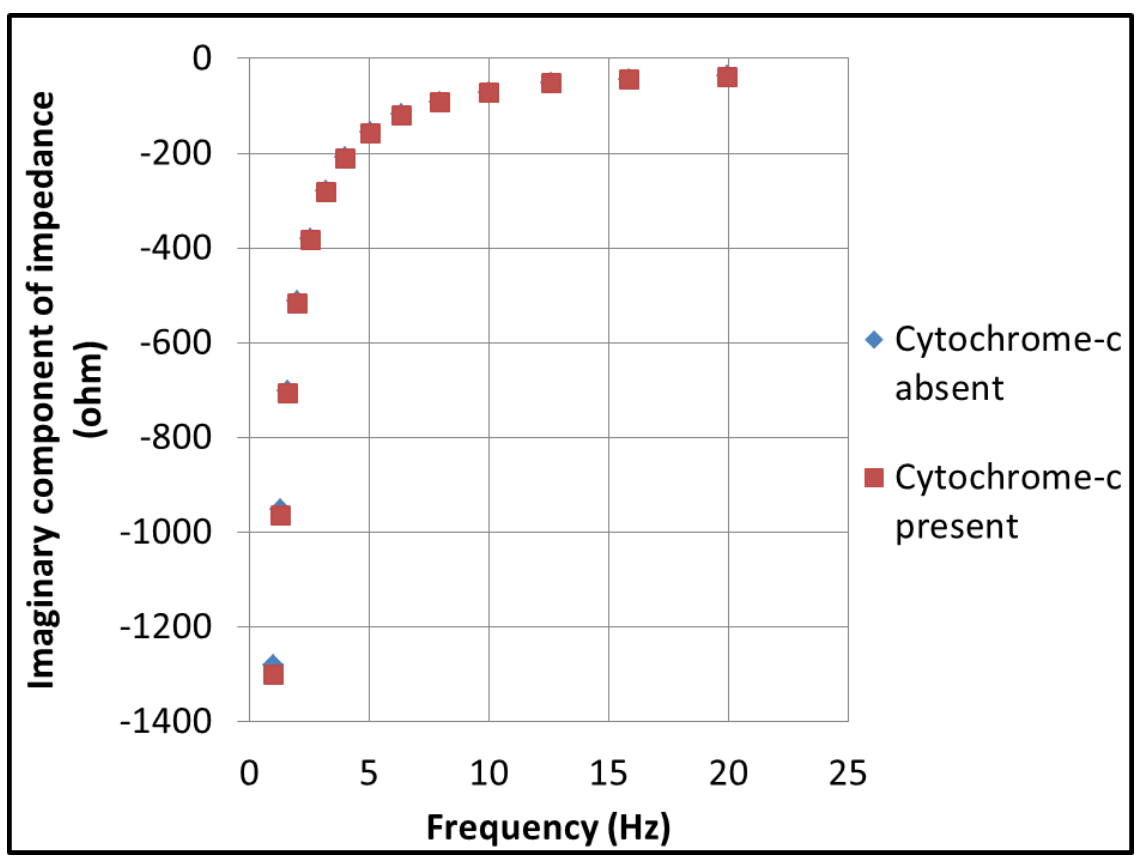

(b)

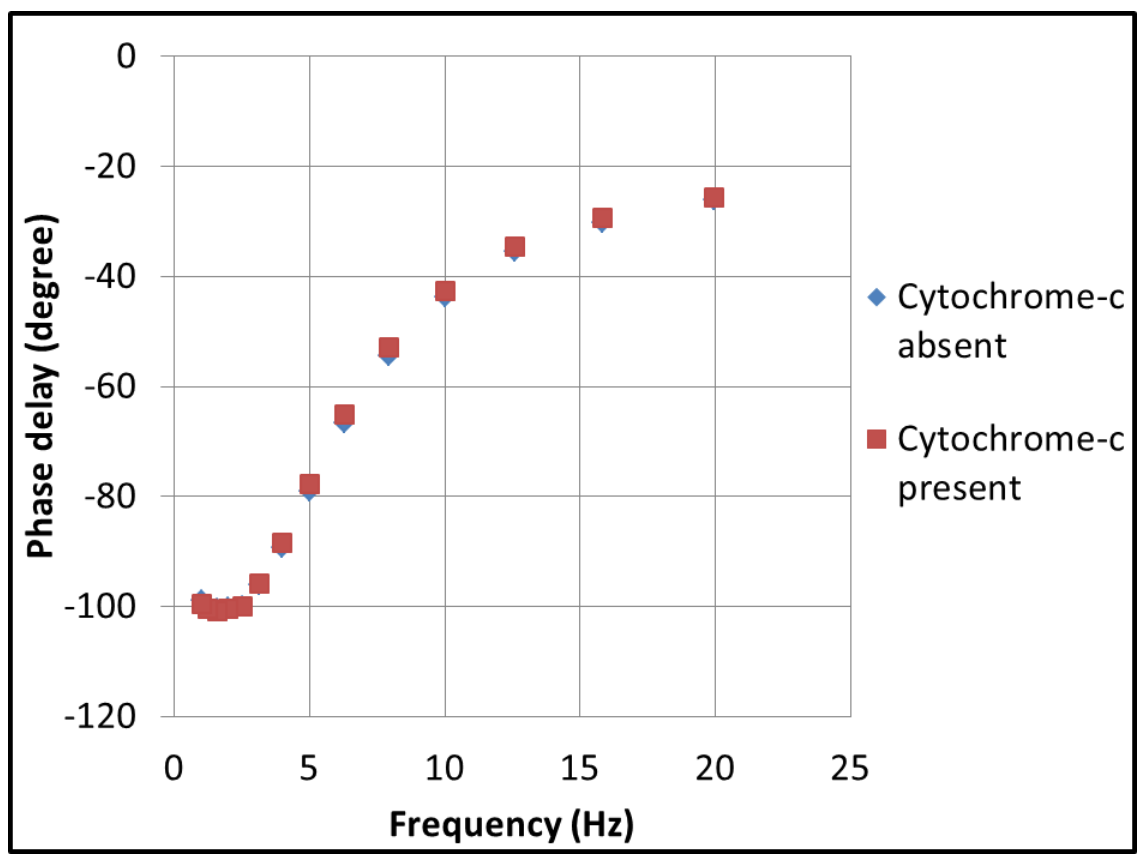

(c)

Figure 5-13: Comparison of electrical impedance results between the electrochemical flow cell without (blue diamond) and with cytochrome c (red square) presence. (a) Real component of the total electrical impedance. (b) 
Imaginary component of the total electrical impedance. (c) Phase delay. Data from $0.08 \mathrm{~V}$ potential DC bias is selected.

The difference between the reference and the protein signals on the purely electrical impedance plots is extremely small. It is unnoticeable of redox reaction for surface-adsorbates at the sub-monolayer coverage. Attempt to retrieve electrochemical information based on pure electrical data is problematic. The electrical impedance data confirms that inability of conventional EIS to perform well under low surface coverage, and justifies the introduction of a novel optical approach based on the EA-SM-IOW to detect and characterize redox adsorbates at low surface concentrations.

Demonstration with different probing wavelengths

As described previously, the OIS using an extremely sensitive EA-SM-IOW can detect analytical signals even when a small fraction of redox species (femtomoles $/ \mathrm{cm}^{2}$ ) were electrochemically modulated. Additional demonstration of the consistency of the approach was carried out by applying different probing wavelengths.

The wavelength at $550 \mathrm{~nm}$ has the strong redox molar absorptivity difference $\Delta \epsilon\left(18 \times 10^{3} \mathrm{M}^{-1} \mathrm{~cm}^{-1}\right)$ for the cytochrome c protein. With the extremely high sensitivity from the EA-SM-IOW, other wavelengths having smaller $\Delta \epsilon$ can be applied for the OIS detection. The wavelength at $570 \mathrm{~nm}$, which also has the 
opposite sign of $\Delta \epsilon\left(-4 \times 10^{3} \mathrm{M}^{-1} \mathrm{~cm}^{-1}\right)$ compared to $550 \mathrm{~nm}$, was selected for this demonstration.

The AC optical data are examined in the complex plane for $550 \mathrm{~nm}$ and 570 $\mathrm{nm}$, as shown in Figure 5-14 (a) and (b), respectively. The data from an AC amplitude modulation of $15 \mathrm{mV}$ at the potential DC bias of $-0.1 \mathrm{~V}$ are used.

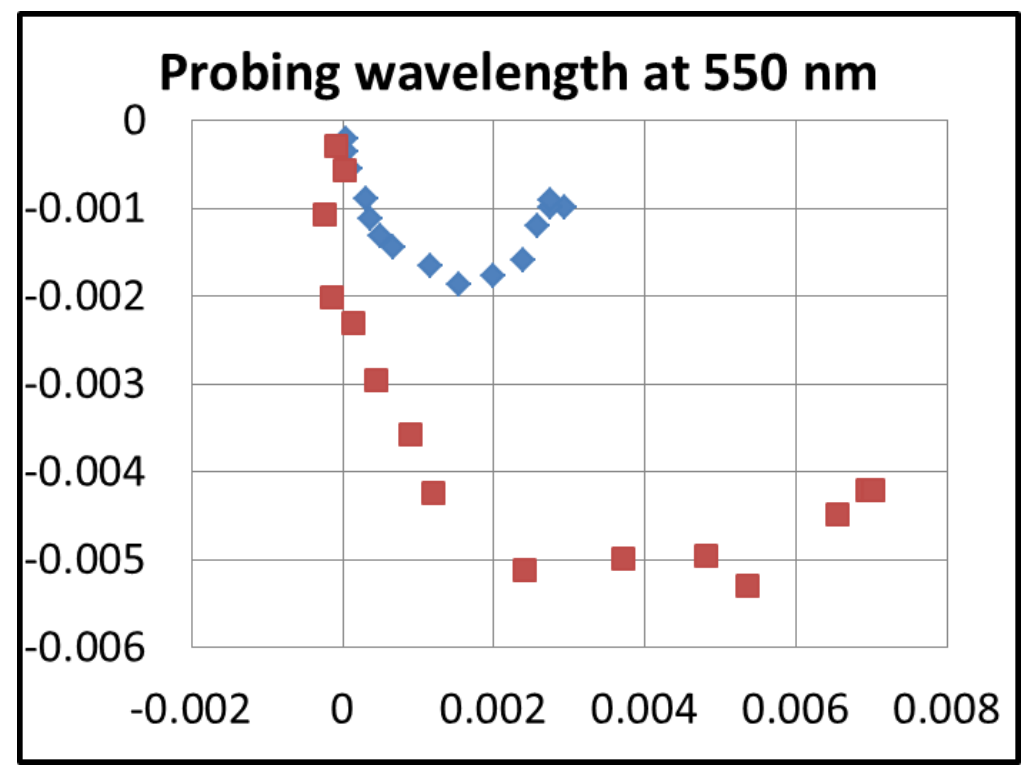

(a) 


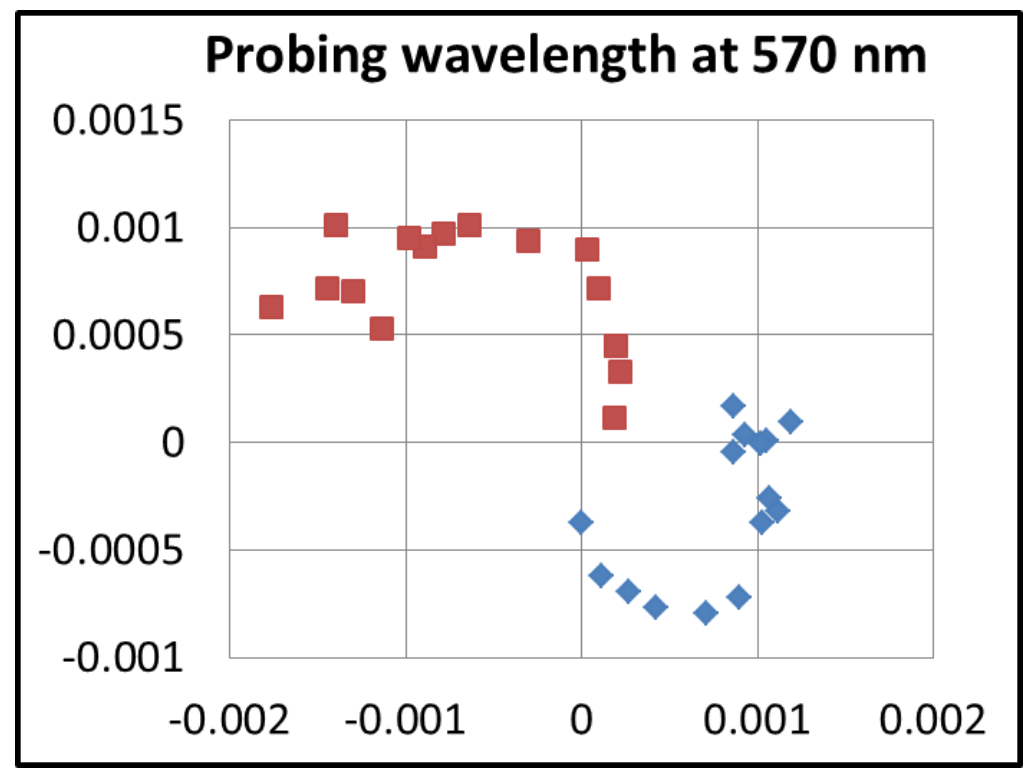

Figure 5-14: The comparison between the reference system and the cytochrome c contained system in optical complex planes for AC absorbance examination. Blue diamond represents the reference optical single and the red square represents the protein contained system for (a) $550 \mathrm{~nm}$ and (b) $570 \mathrm{~nm}$.

For $550 \mathrm{~nm}$, the optical data displayed in the complex plane for the reference signal and the signal for the protein adsorbate show the same sign, and it is the same as shown previously in Figure 5-7. The optical absorbance changes of cytochrome c proteins occur in the same direction as those in ultra-thin ITO film under the potential modulation. As a result, the AC complex plane of cytochrome c protein is larger than the reference complex plane when the potential DC bias is close to the formal potential. For $570 \mathrm{~nm}$ (b), the more negative potential the less absorptive the proteins are. The optical signal in the complex plane for cytochrome c has a negative sign, compared to $550 \mathrm{~nm}$. 
The same linear fitting as in Figure 5-11 for electron transfer rate calculation was used for $550 \mathrm{~nm}$ and $570 \mathrm{~nm}$, as shown in Figure 5-15 (a) and (b) respectively.

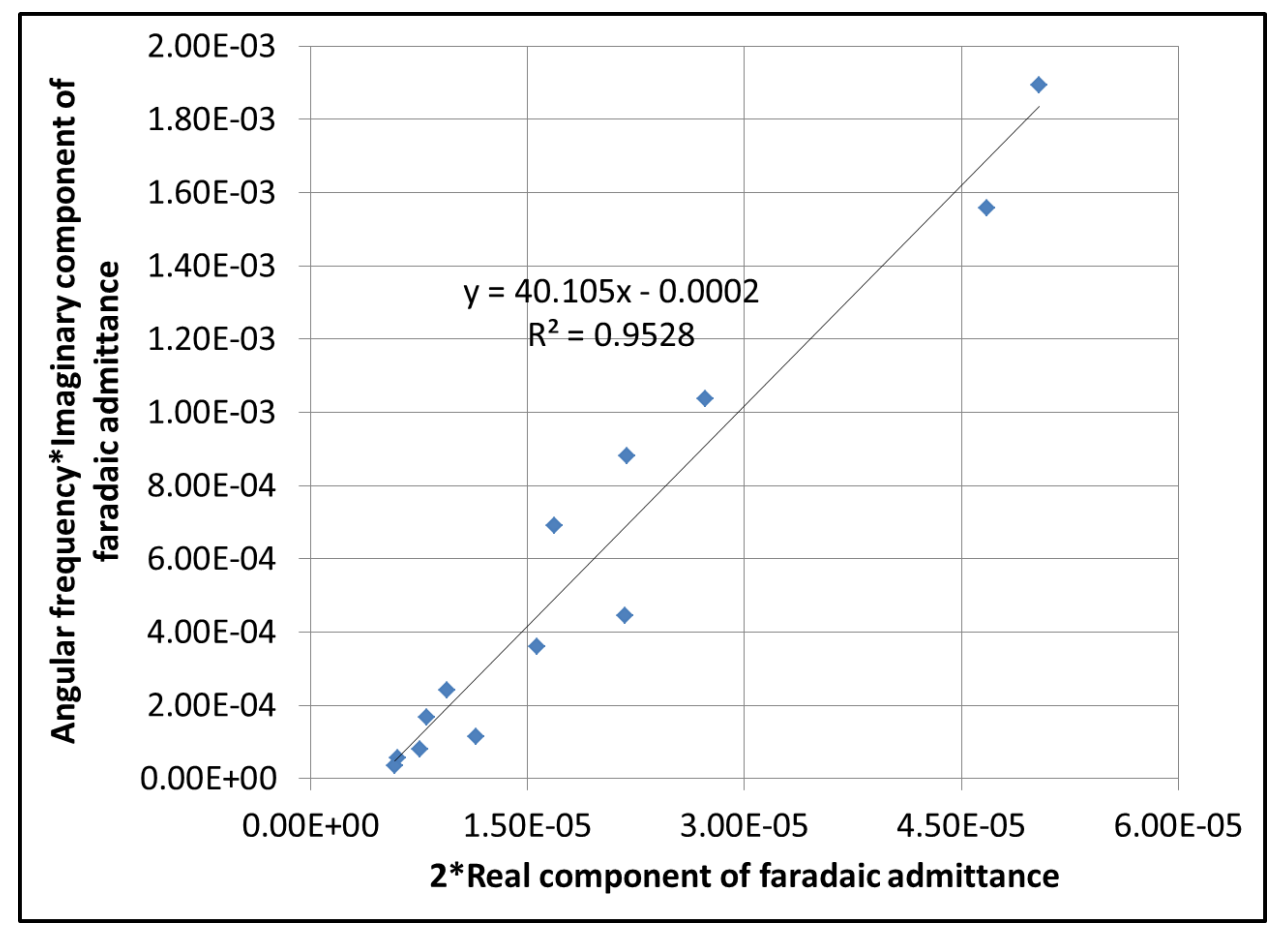

(a) 


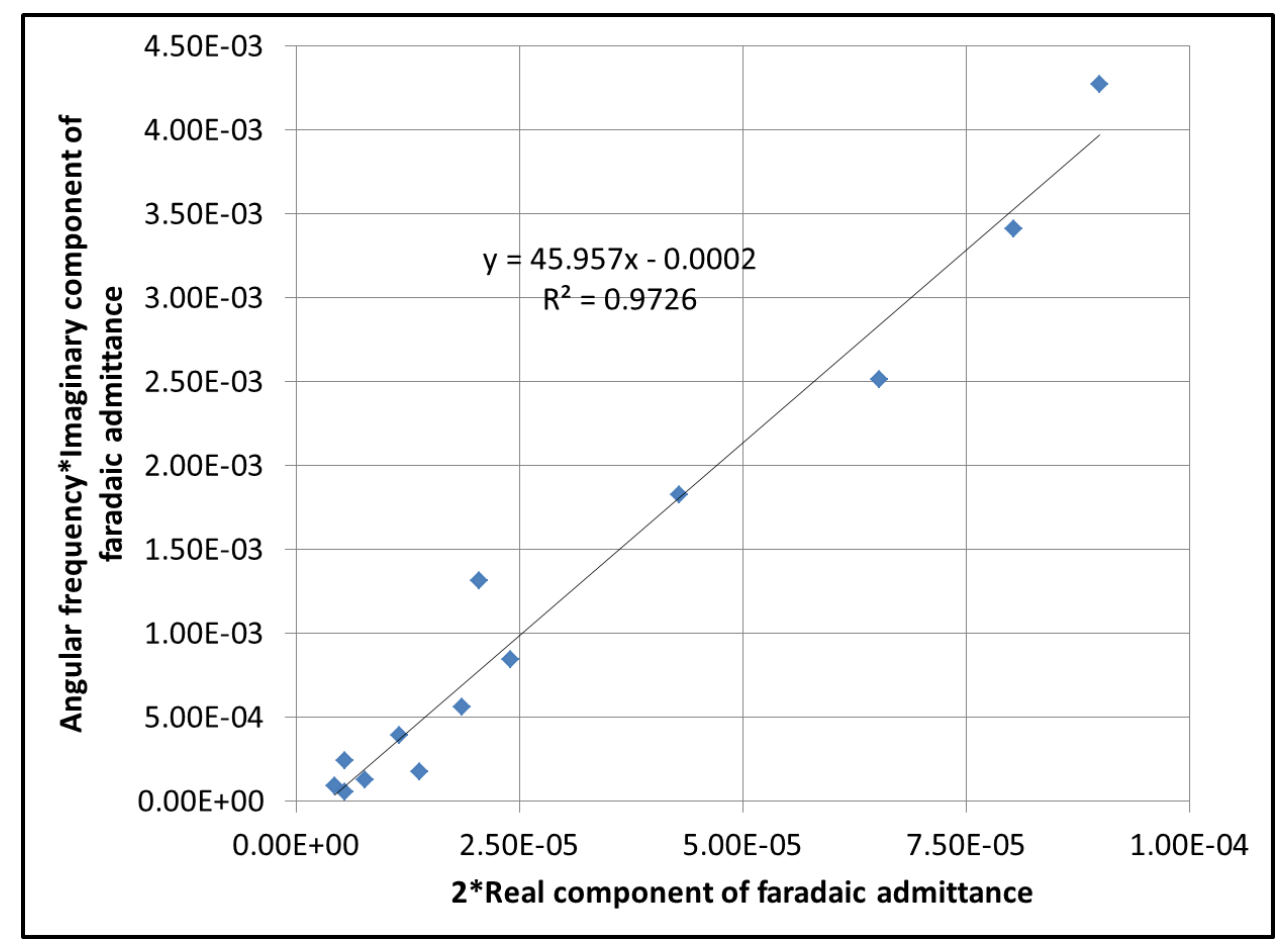

(b)

Figure 5-15: Linear fitting for electron transfer rate calculation for (a) $550 \mathrm{~nm}$ and (b) $570 \mathrm{~nm}$ using optical impedance spectroscopy results.

The electron transfer rates were close for $550 \mathrm{~nm}\left(40 \mathrm{~s}^{-1}\right)$ and $570 \mathrm{~nm}\left(46 \mathrm{~s}^{-}\right.$ $\left.{ }^{1}\right)$. The electron transfer rate for the surface immobilized cytochrome c proteins from this set of test was different than the result from previous section 5.3.4. A different EA-SM-IOW device was employed. Although the device fabrication process was similar, the surface chemistry of the ultra-thin ITO films was unlikely to be identical. 


\section{Conclusions}

The theoretical development of the new methodology for OIS based on the EA-SM-IOW platform was demonstrated. The electrochemical reaction of surface immobilized cytochrome c protein at sub-monolayer level was studied, and experimental results showed the applicability of this novel methodology. The highly sensitive EA-SM-IOW has been proven with an ability in detection and characterization of very low surface density $\left(10^{-15} \mathrm{~mol} / \mathrm{cm}^{2}\right)$ of redox active cytochrome c proteins under AC potential modulations. The information of the electrochemical faradaic current was retrieved from purely optical signals. In addition, based on this novel and simplified analysis, the electron transfer rate of the redox adsorbate, at surface coverage that was beyond the reach of conventional electrical impedance spectroscopy, was experimentally demonstrated. 


\title{
CHAPTER 6
}

\section{APPLICATIONS OF THE ELECTRO-ACTIVE, SINGLE-MODE, INTEGRATED OPTICAL WAVEGUIDE}

\begin{abstract}
As the experimental results in Chapter 5 shows, the optical impedance spectroscopy (OIS) based on an electro-active single-mode integrated optical waveguide (EA-SM-IOW) is extremely sensitive for studying redox reactions of molecular assemblies at sub-monolayer level. With the novel analysis method, the electron transfer rate can be calculated accurately and simply. In this chapter, this technique is used to examine electron transfer rates of cytochrome c proteins at sub-monolayer surface density for various circumstances.
\end{abstract}

Impact of Total Surface Coverage on Cytochrome c Adsorbates

Total surface coverage effects on electron transfer kinetics of surface immobilized cytochrome c proteins under potential modulations are examined. In this set of experiments, various bulk concentrations of cytochrome c are used to achieve different total surface coverage. The maximum surface coverage is still at sub-monolayer level. 


\section{Experimental set-up and procedures}

Both CV potential scan (to determine the total surface coverage) and AC potential modulations (to study the electron transfer kinetics) were applied. For the $\mathrm{CV}$ potential scan (-0.3 $\mathrm{V}$ to $0.1 \mathrm{~V}, 0.02 \mathrm{~V} / \mathrm{s}$ speed), the same experiment set-up as in Section 3.3.1 was used. For OIS measurements, the experiment set-up used in Chapter 3 was improved, as shown in Figure 6-1. By comparing the optical signal to the potential modulation using a lock-in amplifier (Stanford research system), the optical AC amplitude and phase delay were collected by the oscilloscope.

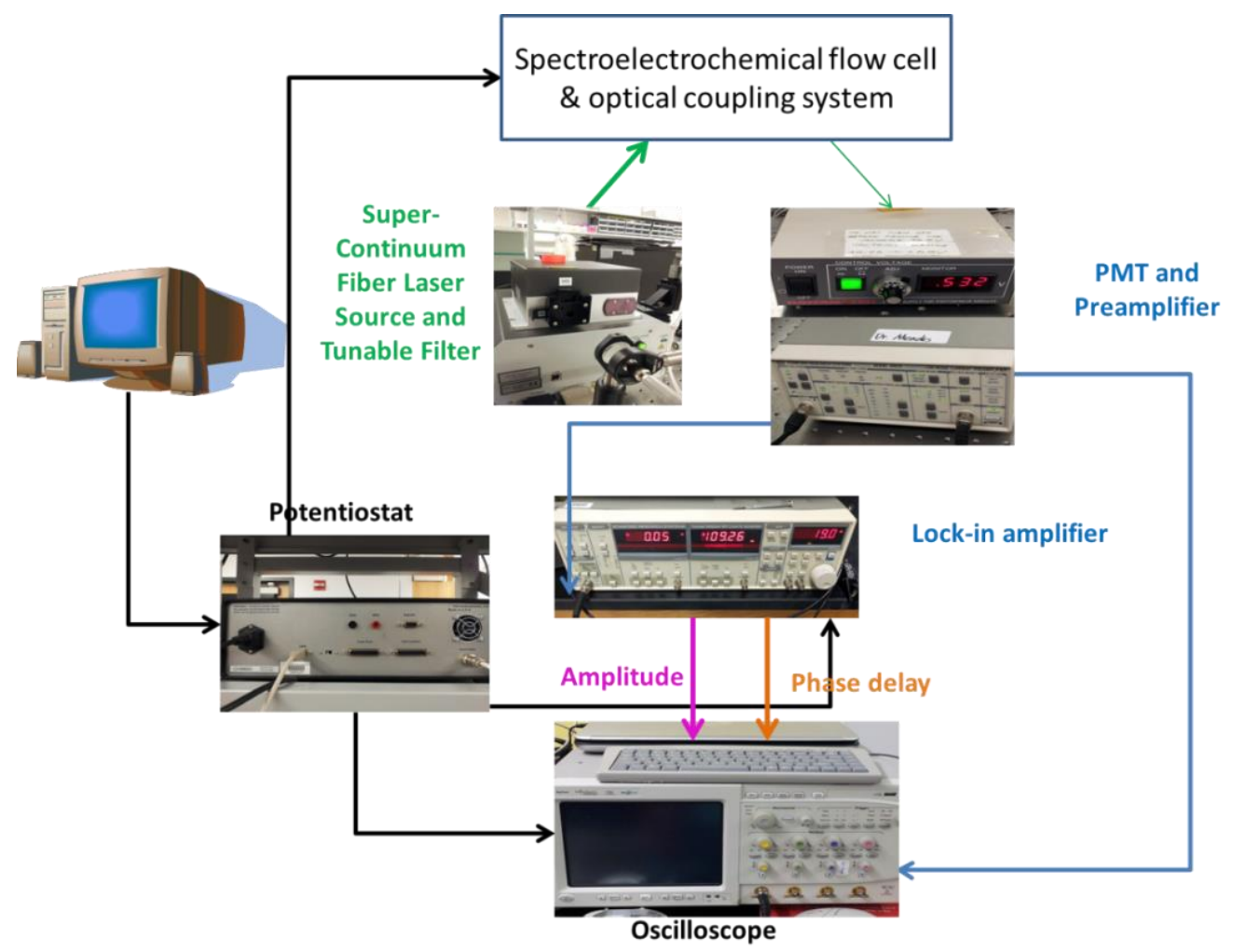

Figure 6-1: Experimental set-up for optical impedance measurement. 
After a cleaning process, the EA-SM-IOW sensor was mounted and the optical coupling was optimized. Then the buffer solution was injected into the flow cell. The optical reference signals were recorded first for both $\mathrm{CV}$ potential scan and $\mathrm{AC}$ impedance potential modulations. Then $2 \mathrm{ml}$ of the lowest bulk concentration of cytochrome c, $25 \mathrm{nM}$, was injected into the flow cell and 30 minutes was used to stabilize the adsorption process. Then a flushing process was used to eliminate floating cytochrome c with buffer solution. Then the same CV potential scan and the AC impedance potential modulations were applied to the system. The same experiment steps, adsorption - flushing with buffer solution - recording optical response under potential modulations, were used for $50 \mathrm{nM}, 75 \mathrm{nM}, 100 \mathrm{nM}$ and $200 \mathrm{nM}$ bulk concentration of cytochrome c protein. This set of measurements was carried out without disassembling the electrochemical flow cell when increasing the cytochrome c protein bulk concentration.

\section{Results of $\mathrm{CV}$ potential scan}

The absorbance from the isosbestic point $557 \mathrm{~nm}$ under the $\mathrm{CV}$ potential scan was examined for each bulk concentration of cytochrome c protein. The surface coverage of the cytochrome c proteins was calculated, as shown in Figure 6-2. The surface coverage was a constant for each bulk concentration of cytochrome $\mathrm{c}$ during the potential scan. The data confirms no net adsorption or desorption of cytochrome c protein under the potential modulation. As expected, the higher the cytochrome $\mathrm{c}$ protein bulk concentration, the higher the surface coverage was resulted. 


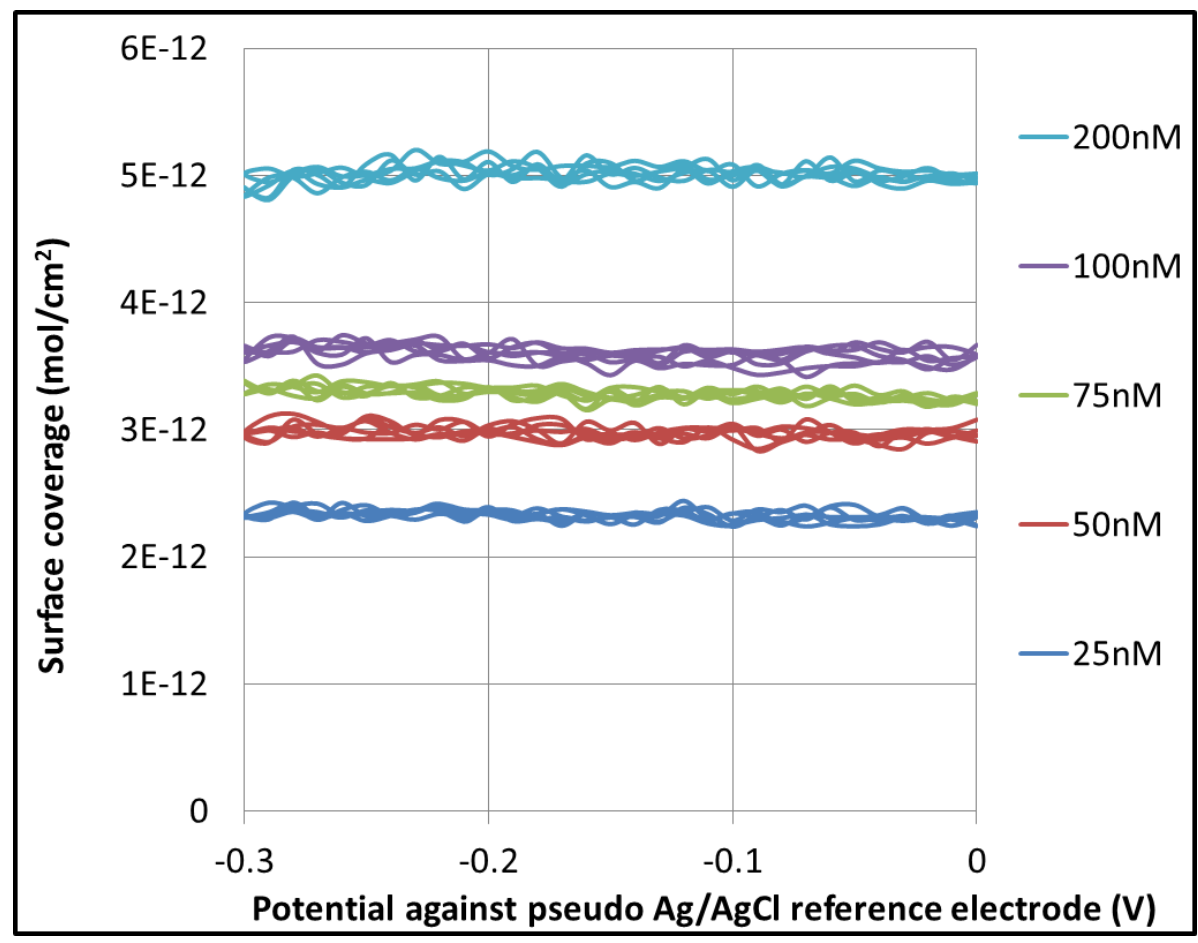

Figure 6-2: Total surface coverage during the CV potential scans monitored by an isosbestic wavelength, $557 \mathrm{~nm}$. The higher the bulk concentration of the cytochrome c protein, the higher total surface coverage is resulted. For one bulk concentration of cytochrome c proteins, the total surface coverage is a constant during the potential modulation.

The Langmuir model was used to obtain information of the saturated surface coverage, $\Gamma_{\text {sat }}$ and the equilibrium adsorption constant, $K_{a d}$, as shown in Equation 61 , where $\Gamma_{b}$ is the surface coverage resulted from a bulk concentration of the protein, $C_{b}$.

$$
\Gamma_{b}=\frac{K_{a d} \Gamma_{s a t} C_{b}}{1+K_{a d} C_{b}}
$$


The experimental results and simulated curves of the total surface coverage against bulk concentration are shown in Figure 6-3. From the fitting results, $\Gamma_{\text {sat }}$ is $(5.9 \pm 0.7)$ picomoles $/ \mathrm{cm}^{2}$ and $K_{a d}$ is $(2.3 \pm 0.8) \times 10^{7} \mathrm{M}^{-1}$.

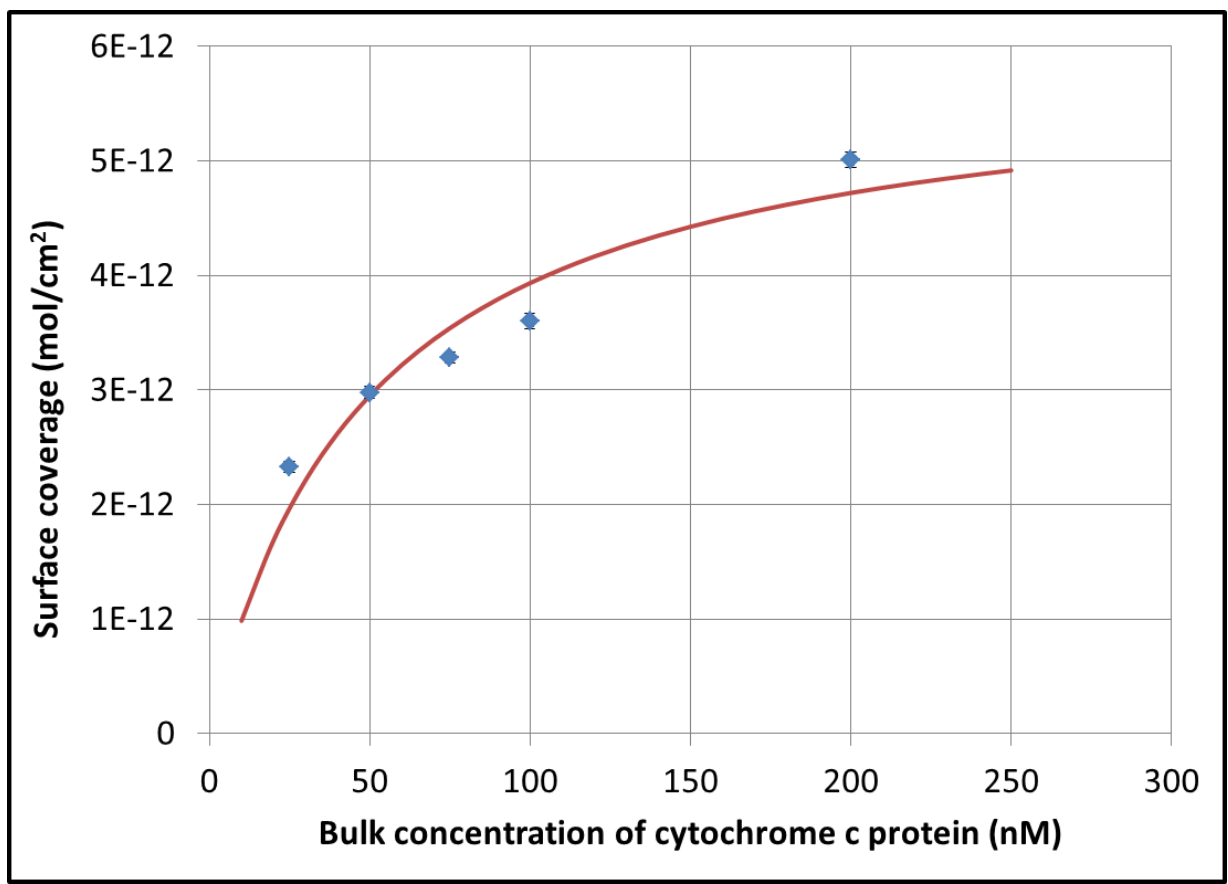

Figure 6-3: Experimental results of the surface coverage under different bulk concentrations with a Langmuir fitting.

Absorbance data from $550 \mathrm{~nm}$ Figure 6-4 (a) were used to reconstruct the faradaic current density profiles for each total surface coverage case, as shown in Figure 6-4 (b). The values of the faradaic current density peak, the full width at half maximum (FWHM) and the formal potential were examined by using Gaussian fit. As expected, the higher surface coverage, the higher the faradaic current density 
was, as shown in Figure 6-4 (b). The average height of the faradaic current density peaks for 6 segments, including both scanning directions (positive and negative), was plotted against the total surface coverage of the cytochrome c proteins, as shown in Figure 6-4 (c). The result from the $200 \mathrm{nM}$ bulk concentration of cytochrome c proteins was not include because the absorbance was too high and may have reach the detection saturation.

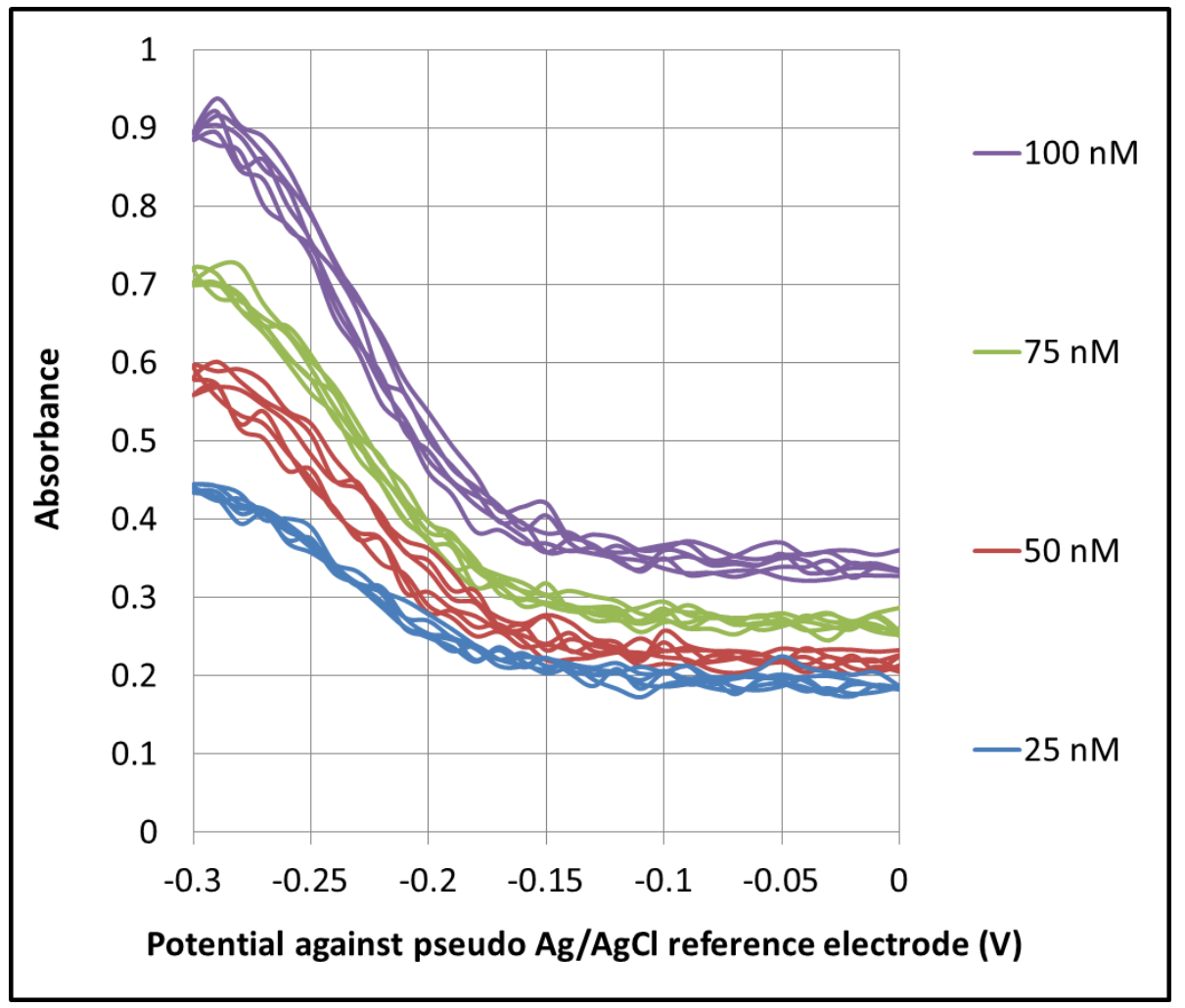

(a) 


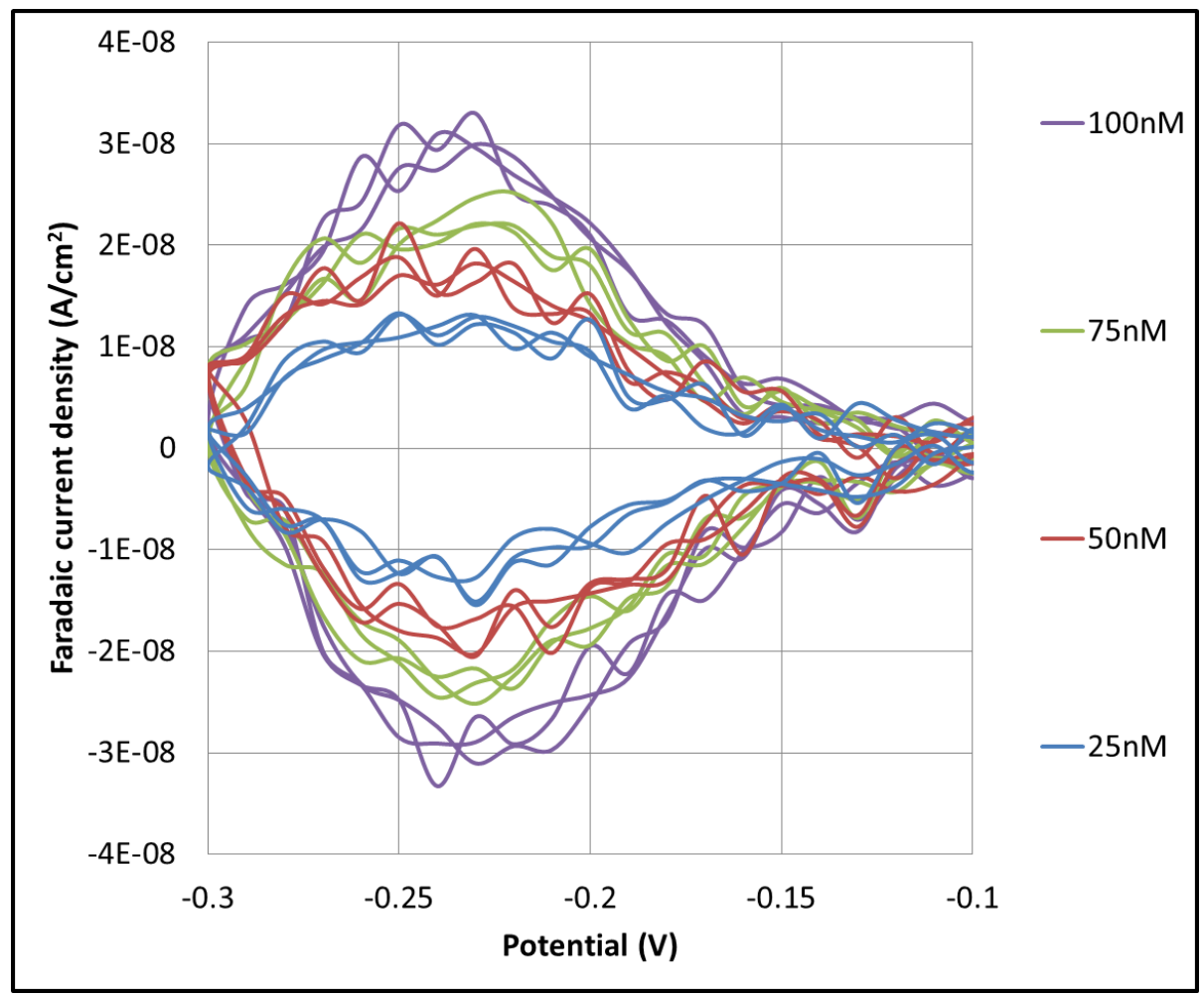

(b)

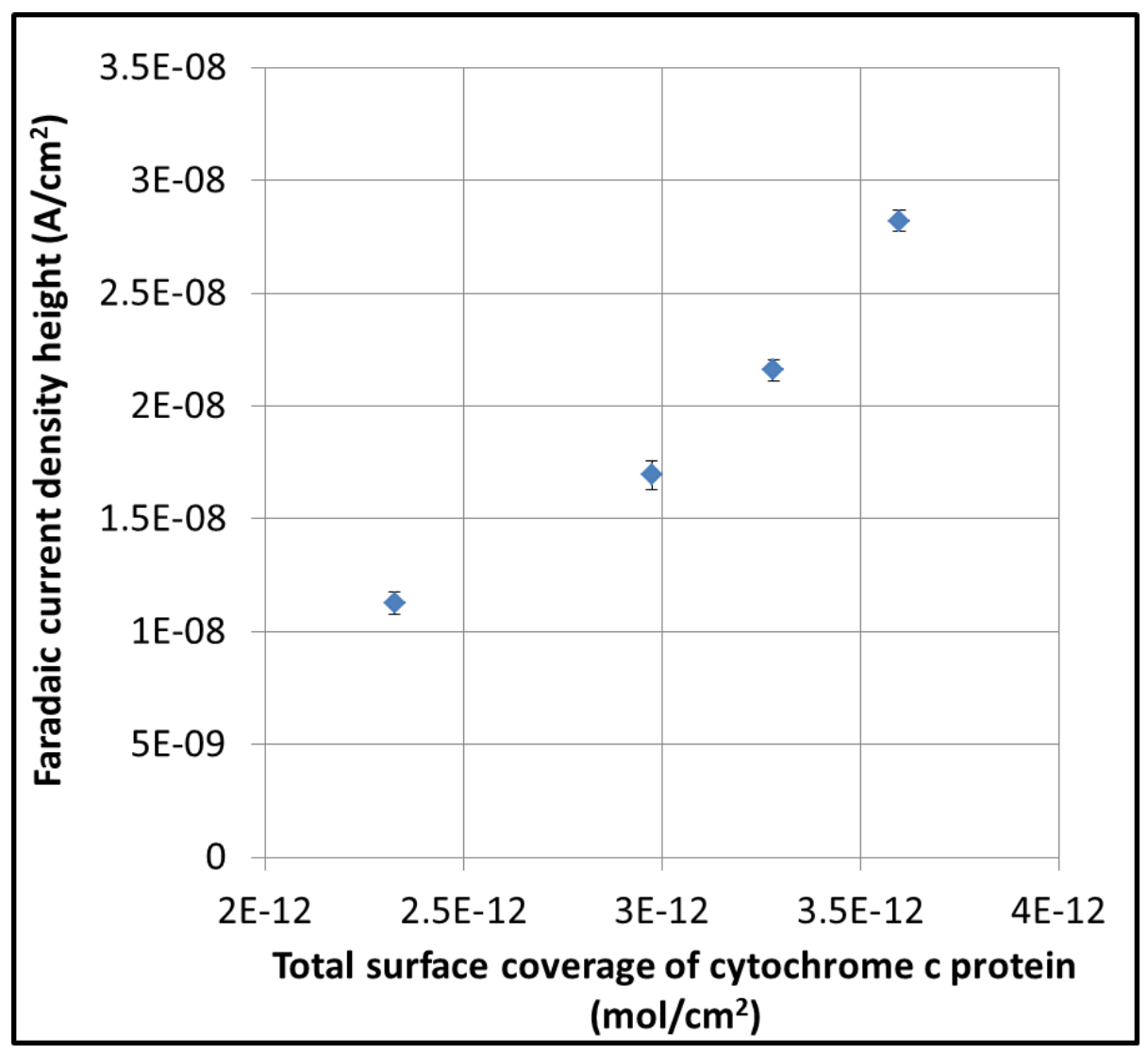

(c) 
Figure 6-4: (a) Absorbance data from $550 \mathrm{~nm}$ wavelength during CV scan for each total surface coverage, (b) reconstructed faradaic current density profiles by using absorbance signals from $550 \mathrm{~nm}$, and (c) average of faradaic current density peaks against total surface coverage under the same CV scan.

The active surface coverage of cytochrome $\mathrm{c}$ was calculated by using the height of average of faradaic current density with Equation 4-5. Based on calculated total surface coverage from $557 \mathrm{~nm}$, the reactive percentage $\left(\Gamma_{a c t} / \Gamma_{\text {tot }}\right)$ of the population the cytochrome c was calculated. In Figure 6-5, the active percentage was plotted against the total surface coverage. The percentage of redox reactive species was not constant. The more cytochrome c proteins on the surface, the more of them were active for the redox reaction.

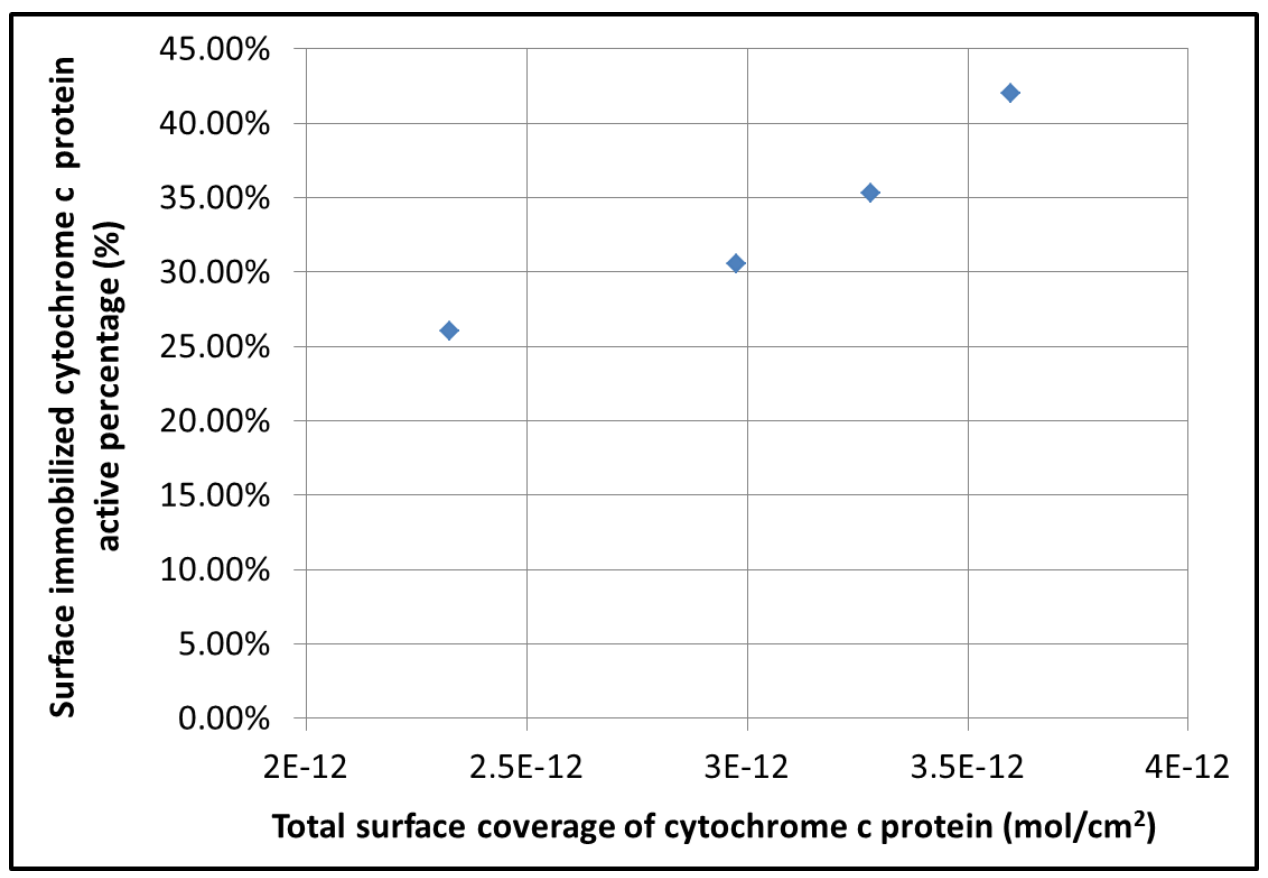


Figure 6-5: Reactive percentage of the surface immobilized cytochrome c against the total surface coverage.

The formal potential and the FWHM were also examined, as shown in Figure 6-6 (a) and (b), respectively. As the standard deviation was rather large for these measurements, no strong correlation of the formal potential or the FWHM with the total surface coverage could be observed.

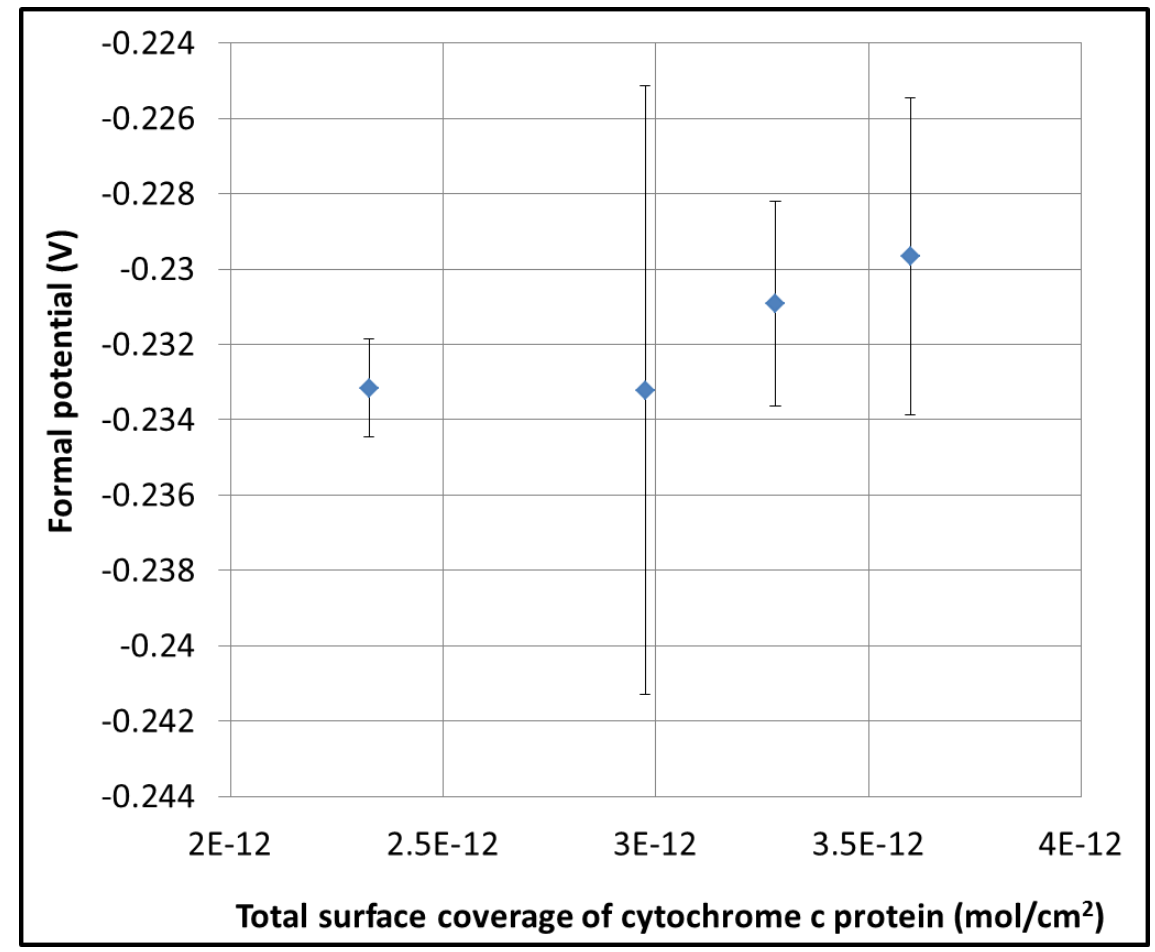

(a) 


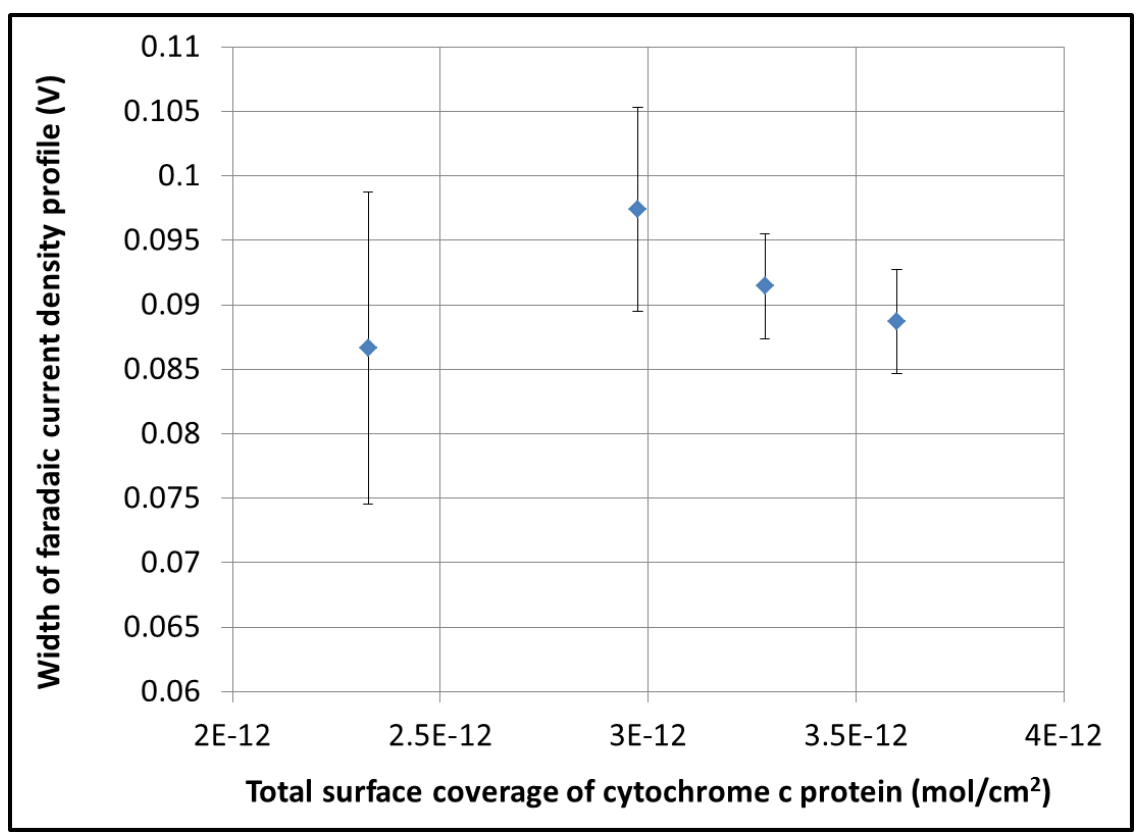

(b)

Figure 6-6: (a) Formal potential and (b) full width at half maximum of the faradaic current density profiles of cytochrome c proteins under different total surface coverage. The average of 6 segments of the CV scan is used with the standard devotion as the error bars.

Results of the optical impedance spectroscopy

For the OIS measurements, the faradaic current density profiles were reconstructed with the $\mathrm{DC}$ and $\mathrm{AC}$ absorbance components from $4 \mathrm{~Hz}$ modulation frequency, as shown in Figure 6-7 (a) and (b) respectively with Gaussian fits. 


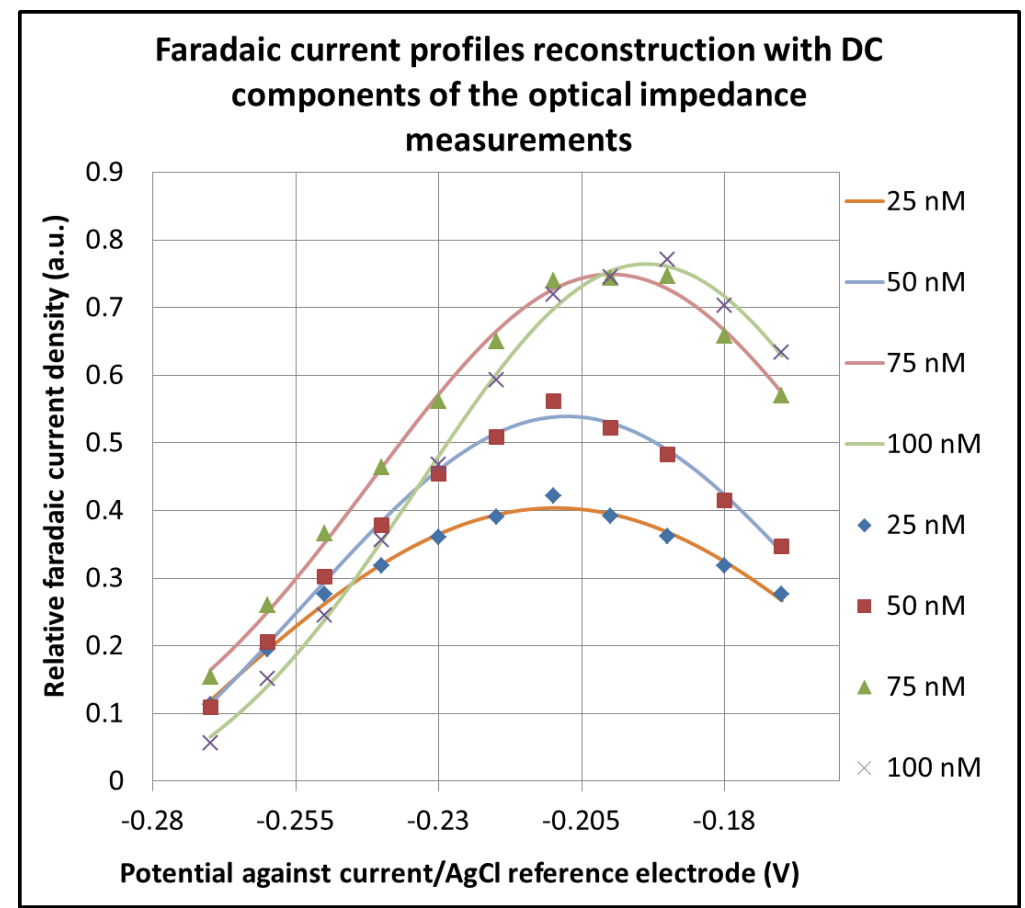

(a)

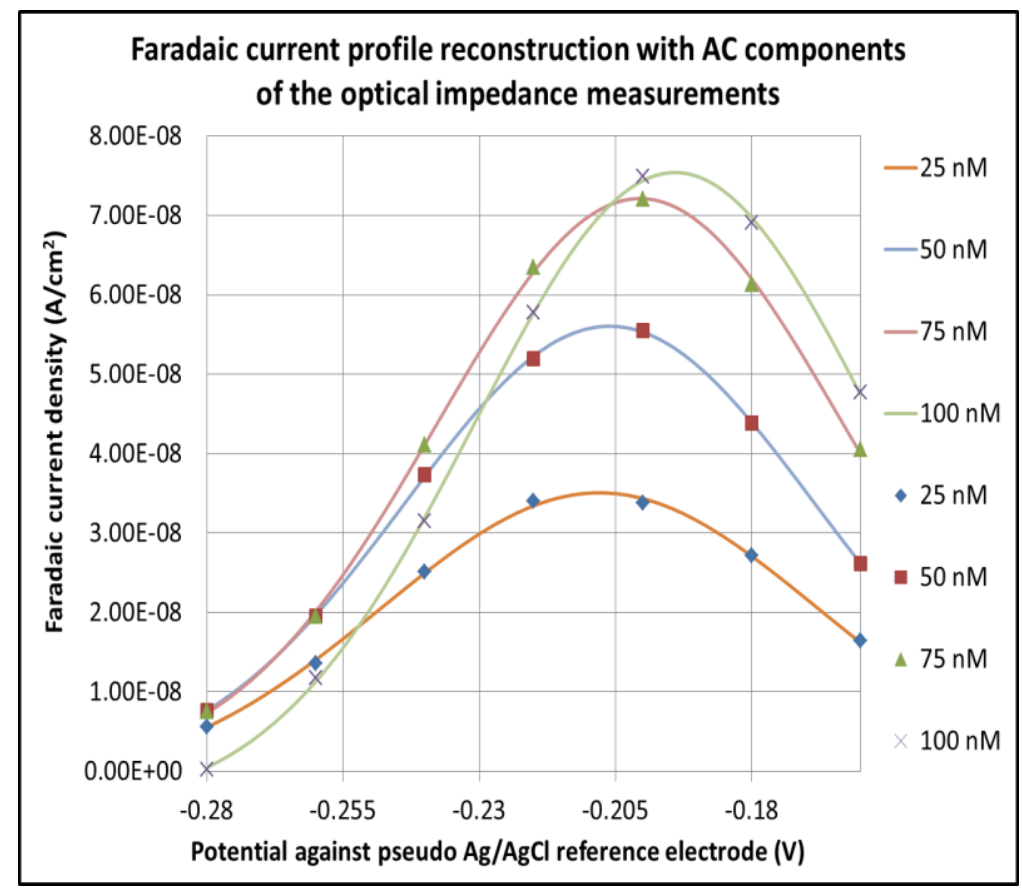

(b)

Figure 6-7: Reconstructed faradaic current density profiles from (a) DC and (b) AC components of absorbance data from OIS measurements. The dotted curves are from experimental results and the solid curves are Gaussian fits. 
With Gaussian fitting results, the formal potential of the faradaic current density profiles reconstructed from the DC and AC components were examined and compared, as shown in Figure 6-8.

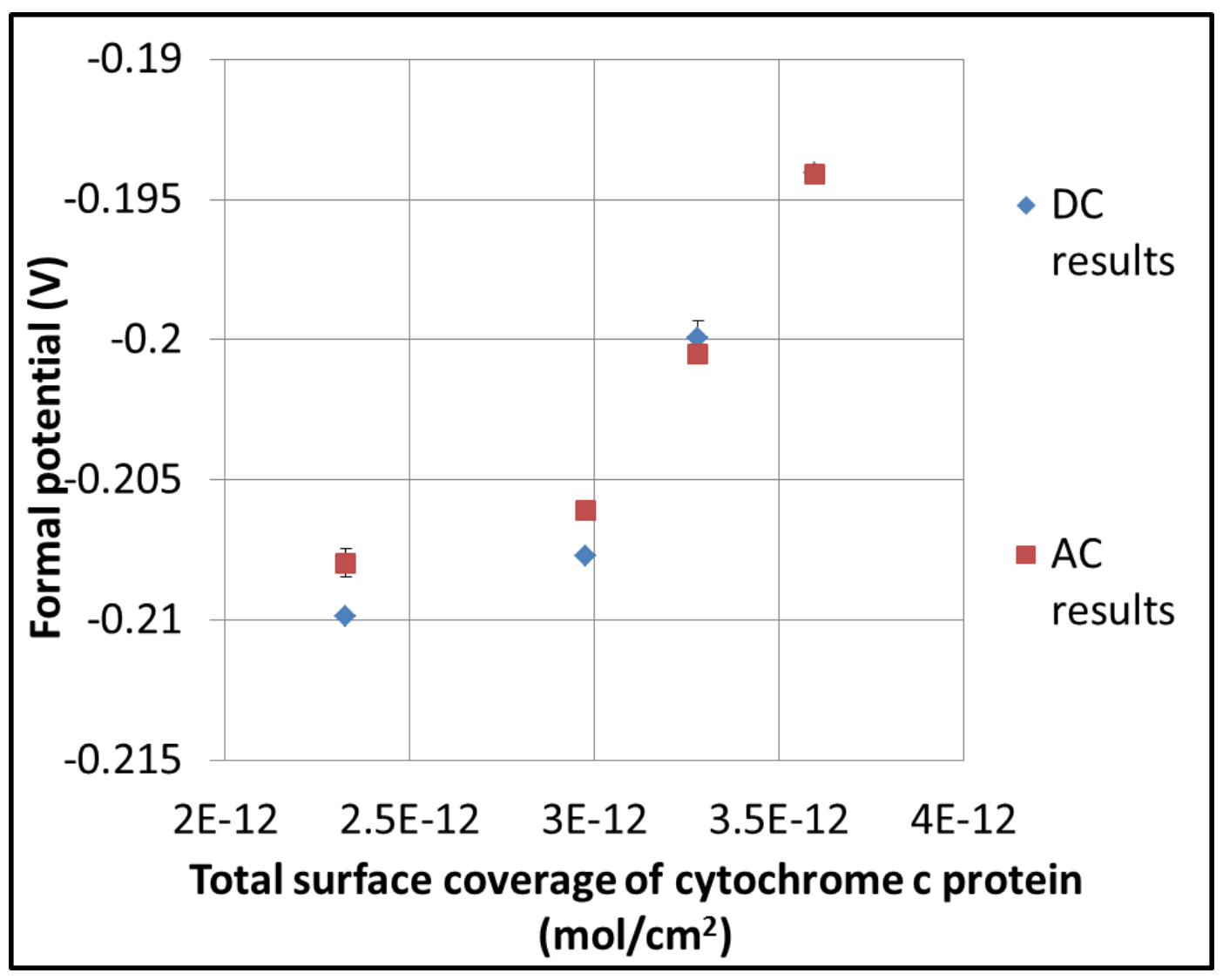

Figure 6-8: Formal potential for cytochrome c proteins redox reactions against total surface coverage. The DC (blue diamond) and AC components (red square) of the optical impedance data at $4 \mathrm{~Hz}$ are used to reconstruct the faradaic current density profile and examined with Gaussian fitting. 
A strong agreement between results from the DC and AC components was observed. In addition, both data showed that the formal potential moved to the positive side when increasing the surface density of cytochrome c proteins on the working electrode, which was consistence with results from reference [200].

Gaussian fitting results for FWHM was examined, as shown in Figure 6-9 (a) for faradaic current density profiles reconstructed from DC components and (b) from AC components. From both plots, we observed that as the surface coverage increased the faradaic current profile became narrower, and these experimental results did not support statements present in reference [200].

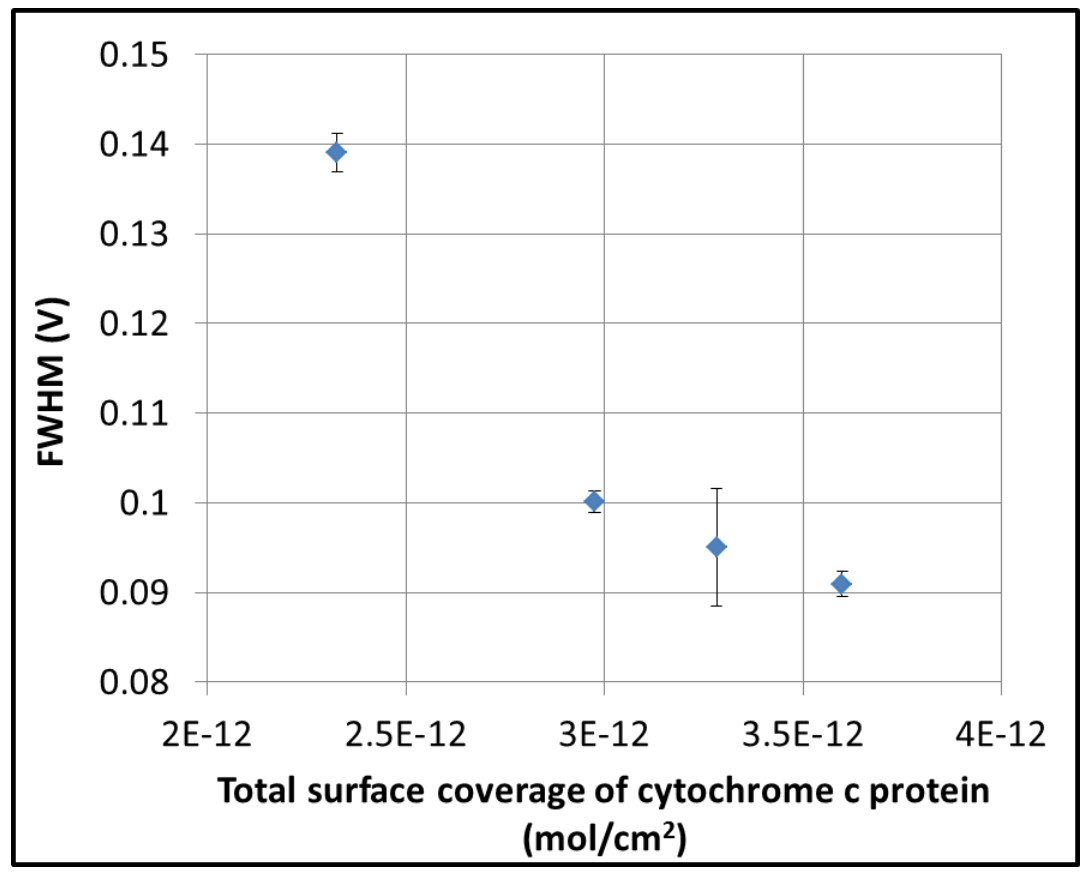

(a) 


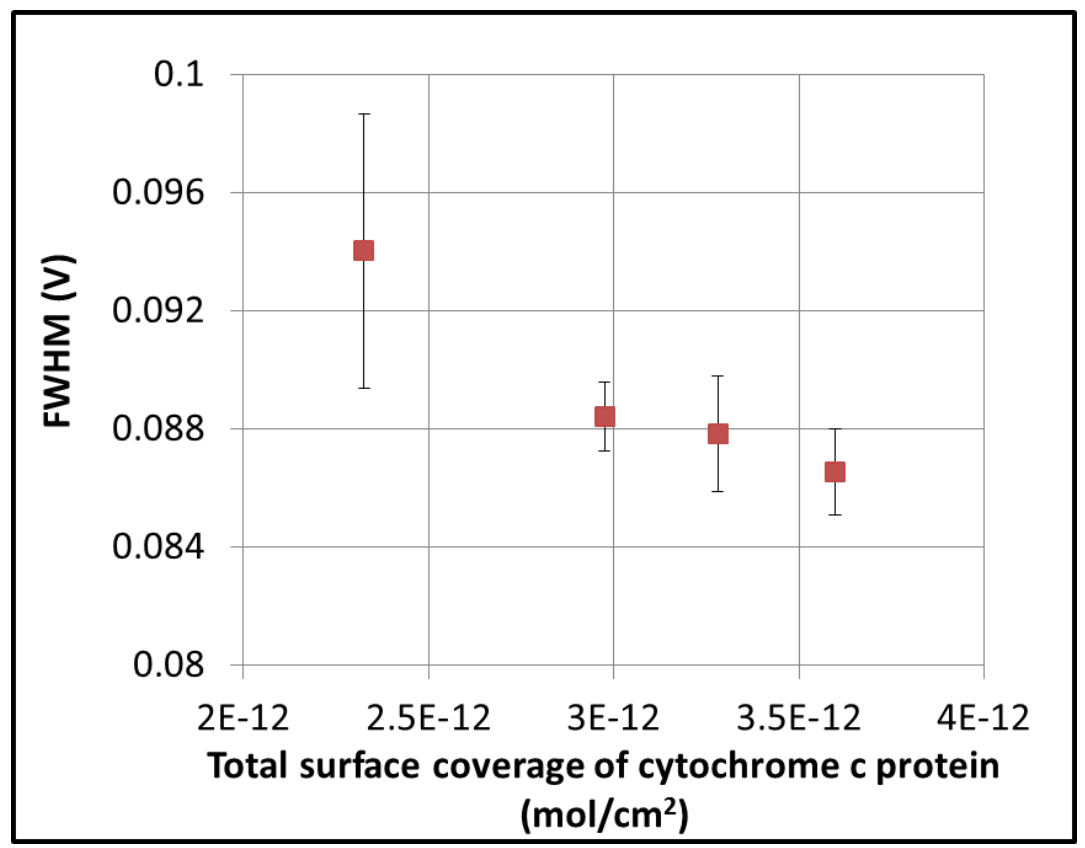

(b)

Figure 6-9: Full width at half maximum of the reconstructed faradaic current profiles from (a) DC and (b) AC components of absorbance data by selecting optical impedance data at $4 \mathrm{~Hz}$ and Gaussian fitting.

Next, the electron transfer rate was calculated based on the novel methodology described in Chapter 5, for each case (total surface coverage). The result is shown in Figure 6-10. 


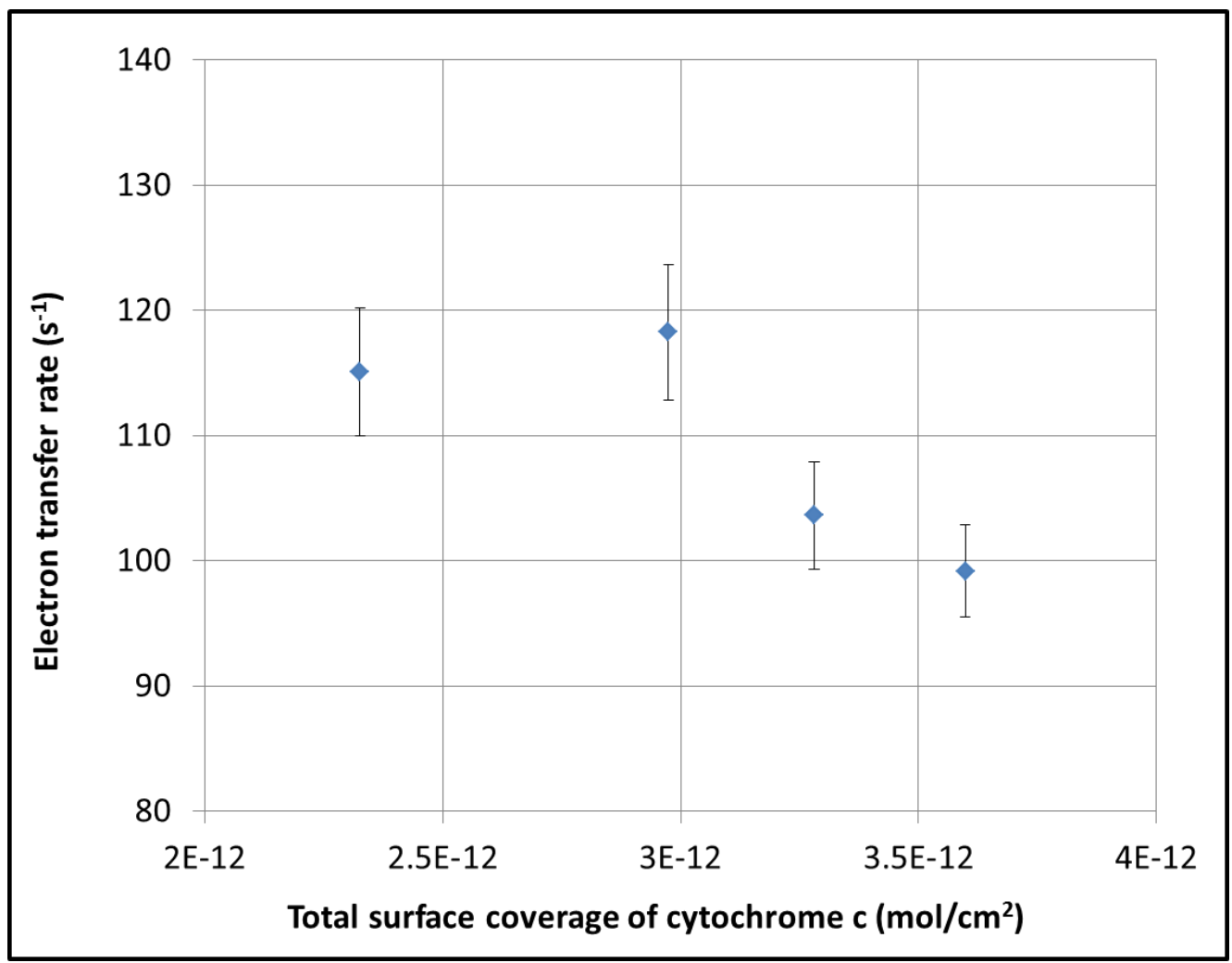

Figure 6-10: Electron transfer rates for different total surface coverage of cytochrome c proteins redox reactions.

When the total surface coverage was low, the electron transfer rate was high and stayed as a constant. When the surface coverage increased, the electron transfer rate was reduced. The same phenomenon has been observed by Edmond F. Bowden and Rose A. Clark [200]. They attributed this result to the different types of binding sites on the ITO surface, strong and weak. According to their hypothesis, a stronger bound site provides faster electron transfer rate. This hypothesis could be used to explain our electron transfer result, which is not likely to be from the protein protein interaction, because the surface coverage of the cytochrome c protein was at 
sub-monolayer level. When the surface concentration is low, cytochrome c molecules are bound to the strong binding sites of the ITO surface. When increasing surface concentration, the strong binding sites are all consumed and the proteins start to bind onto the weaker sites which may create a slower electron transfer rates.

Two reasons could be attributed to explain why a stronger binding site has a faster electron transfer rates. One is a strong bound sites could introduce strong unfolding mechanism to the protein and a reduction in length of electron transfer pathway. With the weak bounding sites, the protein might be partly or not unfolded. The electrons will require additional energy to bridge between the ITO film and the protein heme-center. Another possible explanation is that cytochrome c protein needs time and energy to reorganize its structure for the electron transfer process $[201,202]$. When the protein bounds to the strong sites of the ITO electrode, the structure of the protein is optimized for the electron transfer processes.

\section{Impact of Ionic Strength on Properties of Cytochrome c Adsorbates}

Investigations have been reported in literatures on the adsorption kinetics of the reduced and the oxidized states of cytochrome c proteins on various surfaces under different ionic strength [203-206]. Under the same pH condition, the higher ionic strength, the lower the surface coverage becomes, and the larger equilibrium constant of the adsorption is. Since the attraction force between the cytochrome c protein and the solid surface is primarily an electrostatic force, additional $\mathrm{NaCl}$ ions will directly influence the interaction between cytochrome c proteins and the solid surfaces. The novel methodology of OIS based on an EA-SM-IOW is used to examine 
the ionic strength effects on the electron transfer kinetics of surface immobilized cytochrome c proteins.

\section{Experimental set-up and procedures}

The same experimental set-up in Section 6.1.1 was used, and the wavelength of $570 \mathrm{~nm}$ was selected for the OIS measurements. Ionic strength in buffer solutions was adjusted by concentration the $\mathrm{NaCl}$ salt. The bulk concentration of cytochrome $\mathrm{c}$ proteins in these experiments was fixed at $100 \mathrm{nM}$. The surface of the device was rinsed with DI water, and then cleaned with diluted micro-detergent, rinsed with DI water again and dried by the $\mathrm{N}_{2}$ gas. After the cleaning process, the device was mounted into the electrochemical flow cell. The optical coupling was optimized first, and then a specified $\mathrm{NaCl}$ concentration solution was injected into the electrochemical flow cell. A CV scan from $-0.4 \mathrm{~V}$ to $+0.4 \mathrm{~V}$ was used to stabilize the ITO surface before the optical reference signal was acquired. After this stabilization process, the AC impedance potential modulation was applied and the optical reference signal was recorded. Then a period of 30 minutes was used to stabilize the adsorption of cytochrome c proteins on the surface. Then, the same AC impedance potential modulation was applied to the system and the optical signal was recorded. The same measurement process was applied to all solutions with different $\mathrm{NaCl}$ concentration solutions. For each $\mathrm{NaCl}$ concentration experiment, the same cleaning procedure was used after the previous run to assure that no cytochrome c proteins were left on the surface. 


\section{Electron transfer rate results}

The electron transfer rate was calculated with novel methodology for OIS measurements, and the results were shown in Figure 6-11. For the case of no salt presentation (pure phosphate buffer), the common logarithm of the ratio of the concentration of salt over the bulk concentration of cytochrome $\mathrm{c}$ was set as - 1 . The results showed that when the salt concentration was not sufficiently high enough (from 0 to $1 \mathrm{mM}$ ), the electron transfer rate between the working electrode and cytochrome c proteins was almost as a constant. When the salt concentration was increased (above $10 \mathrm{Mm}$ ), the electron transfer rate was clearly diminished.

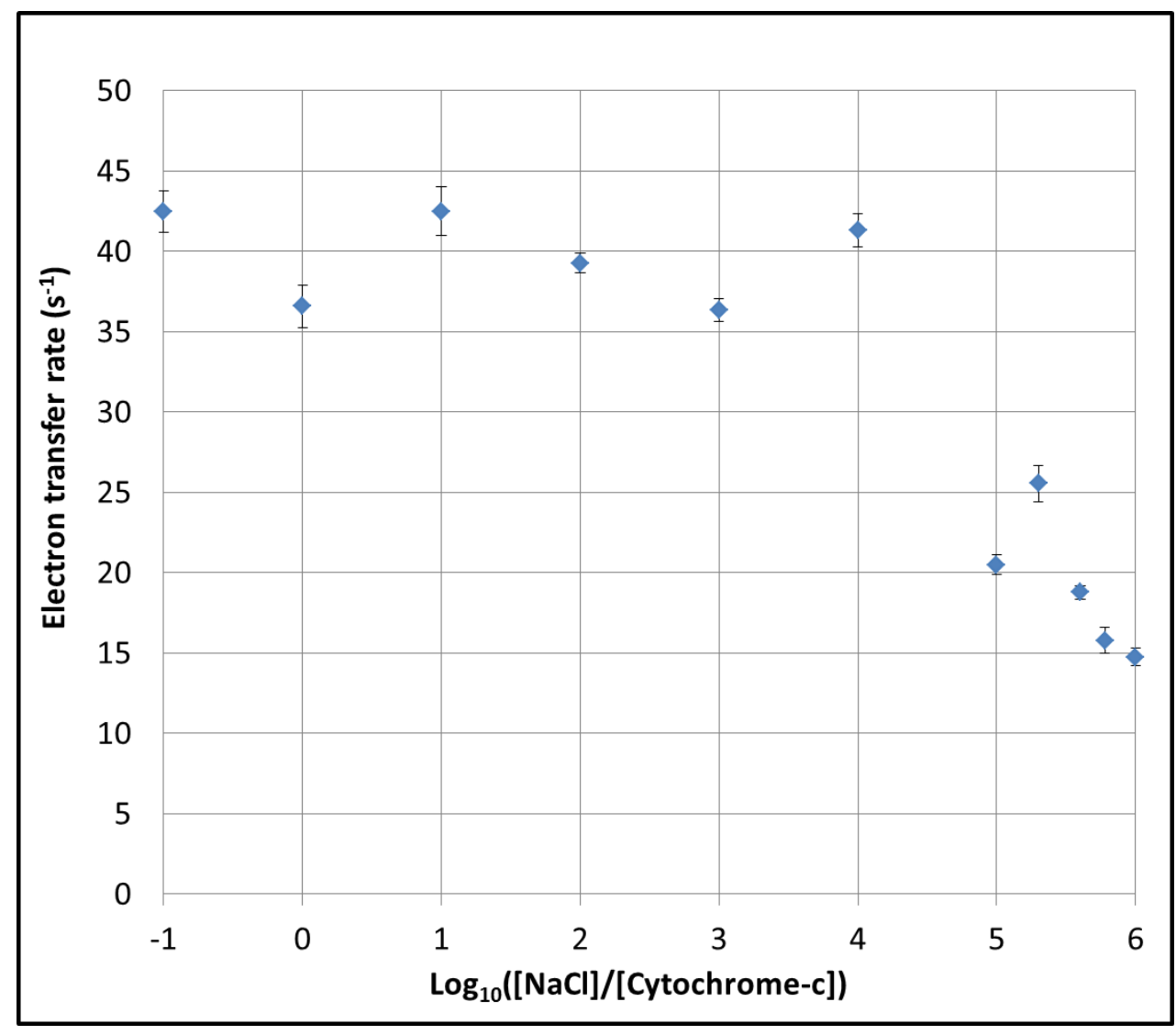


Figure 6-11: Electron transfer rate of cytochrome c protein in different ionic strength solutions. The $\mathrm{x}$-axis is the common logarithm of the ratio the concentration of salt over the bulk concentration of cytochrome $c$. the $\mathrm{x}$ value is set as - 1 for the case that pure phosphate buffer is used.

The observations above can also be understood in terms of the binding strength of the proteins on the ITO surface. Cytochrome c proteins that strongly bind to the ITO surface will feature fast electron transfer rates. When the $\mathrm{NaCl}$ ion strength is not strong enough to break this bound or to strongly shield it, the proteins are not so affected and the electron transfer rate is still high as in buffer with no salt. When the ion strength starts to substantially increase, the salt ions can start to form a shell around the protein and the proteins have less positive net charges. The electrostatic force between the ITO surface and the proteins are reduced. The unfolding of the protein is not at a full degree, and the electron transfer rate starts to decrease. The effects from the ions on cytochrome $\mathrm{c}$ and the ITO surface also are shown in the adoption process. When $\mathrm{NaCl}$ concentration higher is than $100 \mathrm{mM}$, no more surface immobilized cytochrome c can be observed.

\section{Measurements of Electron Transfer Rate with Incident Light at Orthogonal}

\section{Polarization}

Several studies have been done on the orientation of both reduced and oxidized cytochrome c on various surfaces [207-211] and conditions [212-215]. 
Under specially modified surfaces and for specific adsorbates, a net average molecular orientation with a certain order have been created and characterized. Thus, when molecular order is present, light with different polarizations interact optically with different sub-populations of adsorbed species. It has been hypothesized that, if those sub-populations have different electron transfer rates, spectroelectrochemistry with different light polarizations could detect and measure those changes. The experiments described below are aimed to test that hypothesis for the electron transfer rate for the surface immobilized cytochrome c proteins on the bare ITO surface. Polarized light is used for OIS measurements based on an EASM-IOW platform.

\section{Experimental set-up and procedures}

The same experimental set-up was used as in the previous two experiments and the wavelength of $550 \mathrm{~nm}$ was selected. A phase plate was put in front of the super continuum fiber laser source to select the polarization. The same phosphate buffer solution was used to support cytochrome c proteins. When the optimized coupling was achieved, a cyclic voltammetry scan from $-0.4 \mathrm{~V}$ to $0.4 \mathrm{~V}$ was used to stabilize the ITO surface of the device before the optical reference signal was acquired. After this stabilization process, the same AC impedance potential modulations were applied to the electrochemical flow cell and the reference signals with TE and TM polarization were recorded separately. Then a 30 minutes adsorption process was used to stabilize the adsorption of cytochrome c (100 nM bulk concentration) proteins on the surface. After the adsorption, the same AC 
impedance potential modulations were applied to the system with the protein present, and the optical signal data was recorded for both polarized light.

\section{Results of electron transfer rate}

The electron transfer rate was calculated with the novel methodology. The linear fitting results for electron transfer rate for both polarizations were shown in Figure 6-12 (a) and (b).

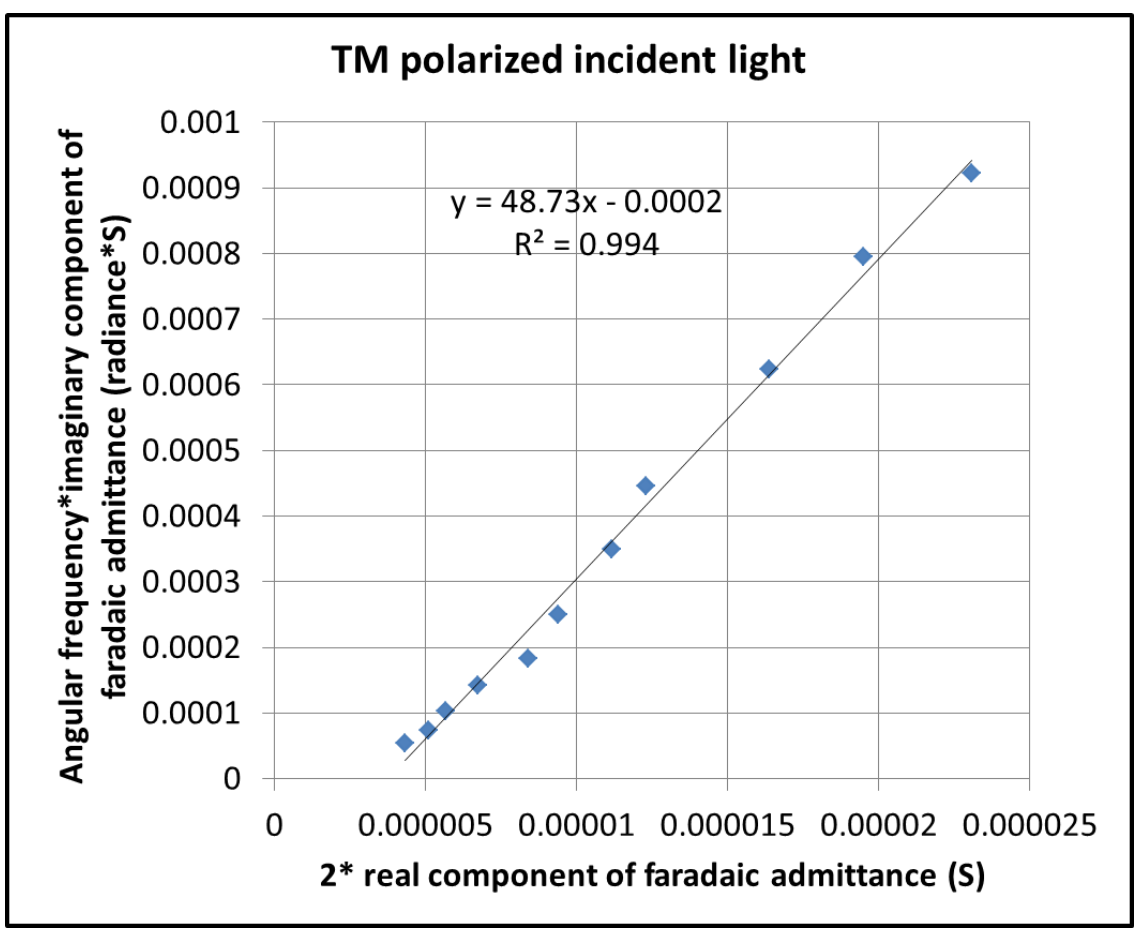

(a) 


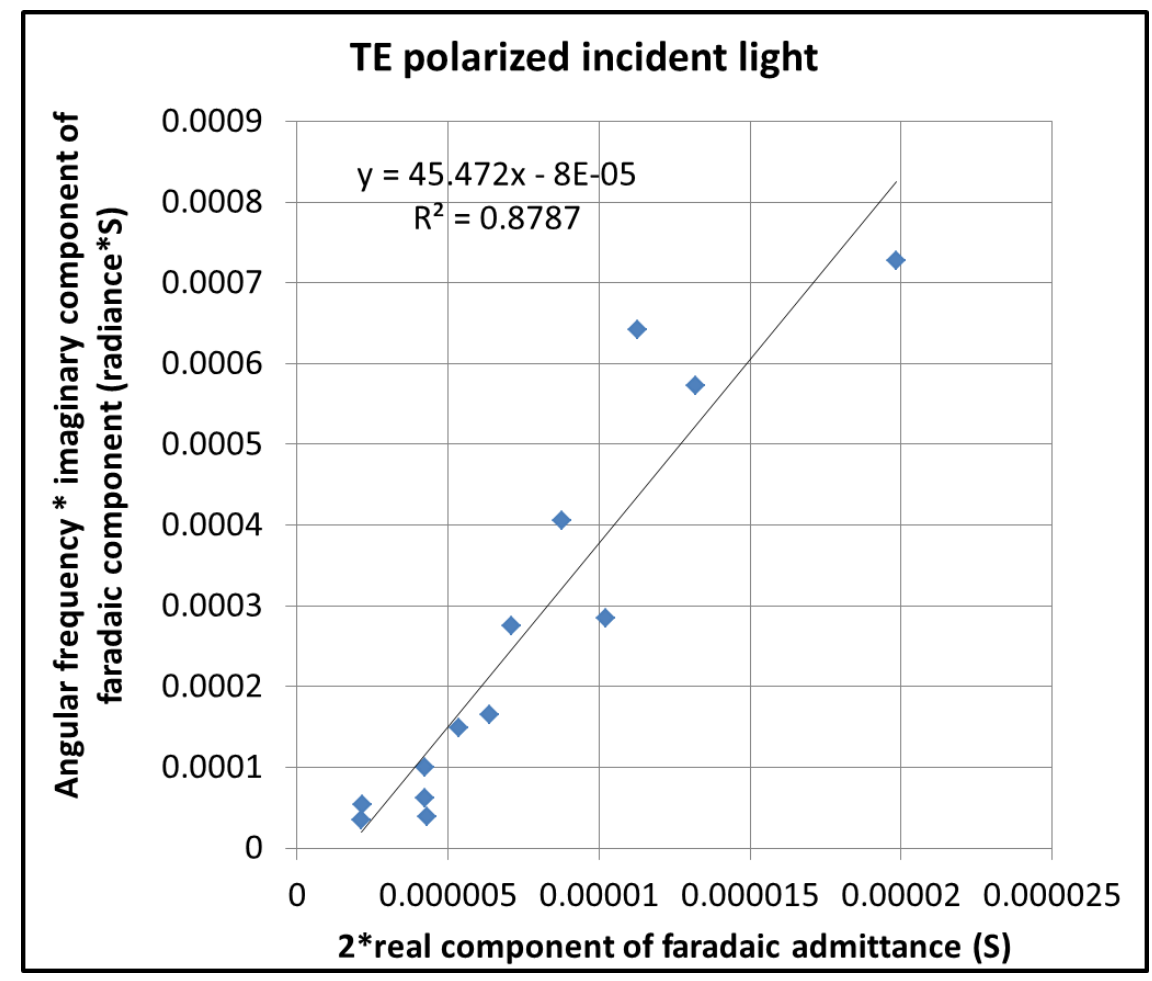

(b)

Figure 6-12: Electron transfer rate calculation for both (a) TM and (b) TE polarized incident light. The electron transfer rate is represented by the slope of the linear fit.

The electron transfer rate was almost the same for TM $\left(49 \mathrm{~s}^{-1}\right)$ and TE $\left(45 \mathrm{~s}^{-1}\right)$ polarizations. This result contradicted previous data published in the literature that claims asymmetry on the electron transfer rate of cytochrome $\mathrm{c}$ on an ITO surface for different polarizations [213]. However our results were consistent with the fact that the molecular order of cytochrome $\mathrm{c}$ on a bare ITO surface is fairly negligible [208].

Although, the experimental results do not show an asymmetry in this particular case, the technique described here is powerful and useful for further 
investigations in the electron transfer kinetics of various molecular assemblies at specific surfaces.

\section{Conclusions}

The EA-SM-IOW was applied in studies of the electron transfer processes for surface adsorbed cytochrome c protein at sub-monolayer level in several different environments. The tested surface densities of redox species were well below the reach of conventional electrochemistry and spectroelectrochemistry techniques, such as the electro-reflectance and potential-modulated transmittance. With the novel methodology for the OIS based on the EA-SM-OW described in Chapter 5, the effects from the total surface coverage and ionic strength on the electron transfer process between cytochrome c proteins and the ITO surface were examined. The higher the surface coverage of the protein, the slower the rate was observed. Similar result was obtained for the effects from ionic strength studies, where the higher the concentration of $\mathrm{NaCl}$ salt in the solution, the slower the electron transfer rate was. These phenomena can be attributed to the bound sites on the ITO surface. Stronger bounding sites facilitate the electron transfer between the protein and the electrode. For weaker bound sites, additional energy is needed for the electron transfer process to go forward. 


\title{
CHAPTER 7
}

\section{COUPLED PLASMON WAVEGUIDE RESONANCE SENSOR: DESIGN AND}

\author{
FABRICATION
}

\author{
Introduction
}

Surface plasmon resonance (SPR) is a highly sensitive analytical technique that is considered by many as a gold standard for the detection of surface binding events. It has been employed in research labs worldwide as a high-end tool for the development of new pharmaceutical drugs. Different variations and methods have been applied to improve its detection limit. One of the modifications is the coupled plasmon waveguide resonance (CPWR) with a dielectric waveguide coated on top of a noble metallic layer. Although theoretical calculations have predicted superior performance of a CPWR over the conventional SPR, the various experimental implementations of CPWR reported in the literatures were not superior to the standard SPR. Either the structure was not properly designed or the quality of the fabricated structure was insufficient to reach a strong optical resonance. A major issue has been the high transparency needed for the dielectric layer to be grown over a noble metal layer. In the following section, the design, fabrication and characterization of a CPWR sensor, based on a high quality of aluminum oxide 
waveguide layer and a silver thin layer, are described and shown to perform better than conventional SPR.

\section{Design of the CPWR Sensor}

A criterion was defined to compare the traditional SPR and CPWR performances in aqueous environment. The best architectures of SPR and CPWR were tested theoretically for bulk refractive index change and molecular thin film adsorption.

\section{Definition of resolution}

A criterion for resolution as the refractive index change, $\Delta n$, is defined in Equation 7-1.

$$
\Delta n=\frac{\Delta \theta}{\partial \theta / \partial n}
$$

A typical reflectivity for a SPR sensor under angular interrogation is shown in Figure $7-1$, where $\Delta \theta$ is the full-width at the half maximum (FWHM) of the resonance, $R_{c}$ is the reflectivity at the critical angle, and $R_{\min }$ is the minimum reflectivity at the resonance angle. When a change of refractive index, $\partial n$, happens next to the device interface, the resonance angle is shifted by $\partial \theta$. For a given refractive index change, a narrower and deeper profile with a large shift in the angle of minimum reflectance is preferred to improve the limit of detection. 


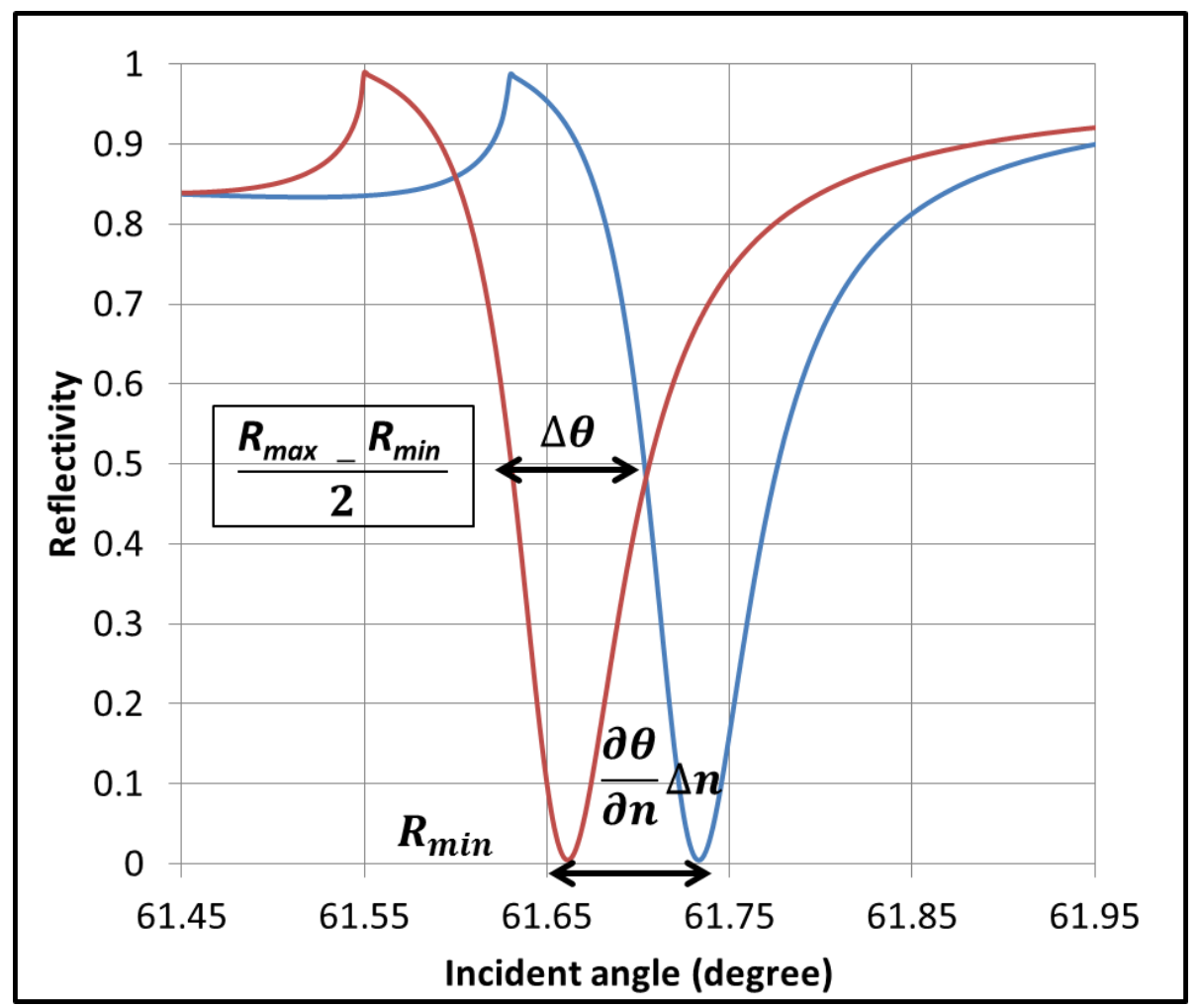

Figure 7-1: Demonstration of the parameters in the definition of resolution for both techniques.

Comparison between CPWR and SPR models

The performance of the CPWR and SPR were compared with their best theoretical configurations. A Mathematic program based on the transfer matrix method was used to simulate the reflectivity versus the incident angle (Appendix 9). A $633 \mathrm{~nm}$ incident light beam was used as the light source for the simulation. For a CPWR, the thickness of each layer and calibrated refractive index are as follows: $t_{\text {silver }}=36 \mathrm{~nm}, n_{\text {silver }}=0.09-4.1 \mathrm{I}, t_{\text {silica }}=5 \mathrm{~nm}, n_{\text {silica }}=1.46$ and $t_{\text {alumina }}=$ $140 \mathrm{~nm}, n_{\text {alumina }}=1.63$. The ideal aluminum oxide thickness was designed just 
above the cutoff thickness to support only the first fundamental TE polarized guided mode. The evanescent field was maximized at the interface when compared to a multimode waveguide. For the best performance for a silver thin film based SPR, $t_{\text {silver }}=52 \mathrm{~nm}$. The CPWR and the SPR were designed for an aqueous environment.

The simulated reflectivity against the incident angle was plotted for both sensors in an aqueous solution $\left(n_{\text {water }}=1.33211\right)$, as shown in Figure 7-2.

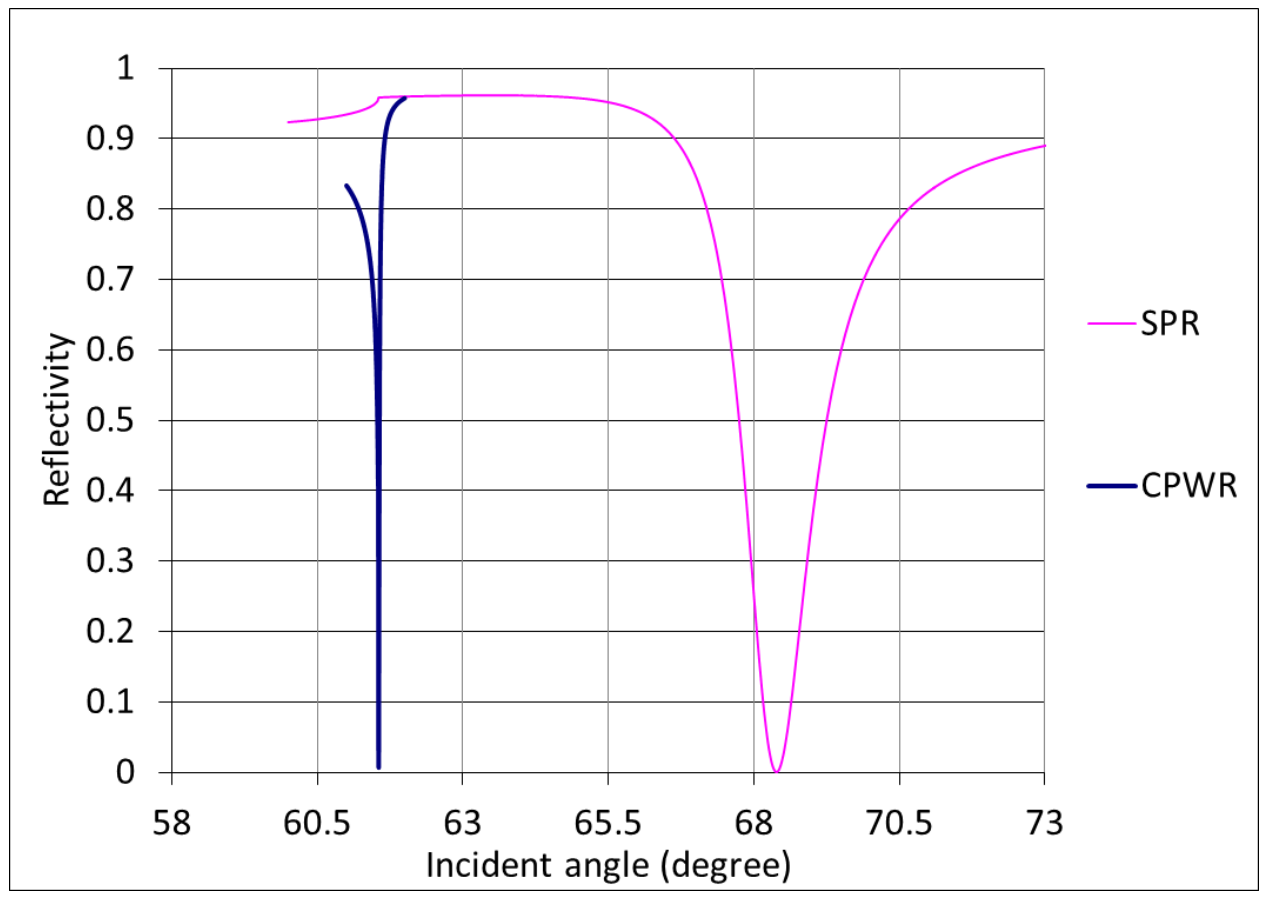

Figure 7-2: Calculated reflectivity curves for CPWR and SPR working in water solution. CPWR results a much narrower curve than the SPR. The best configuration for each sensor is used. 
The angular width of the CPWR is nearly 60 times narrower than that of the SPR. From the definition of the resolution, the resonance angular shift under a refractive index change also plays an important role. Two cases are compared for their performances. One is the bulk refractive index change. And the other one is a refractive index change introduced by a layer of surface immobilized molecular thin film.

\section{Refractive index change in bulk cladding medium}

The bulk solution refractive index change was set from $n_{\text {out }, 1}=1.33211$ to $n_{\text {out }, 2}=1.33311$, which introduced a $\delta n=0.001$ refractive index change Simulations of the reflectivity profiles for the SPR and the CPWR sensor are shown in Figure 7-3 (a) and (b) respectively.

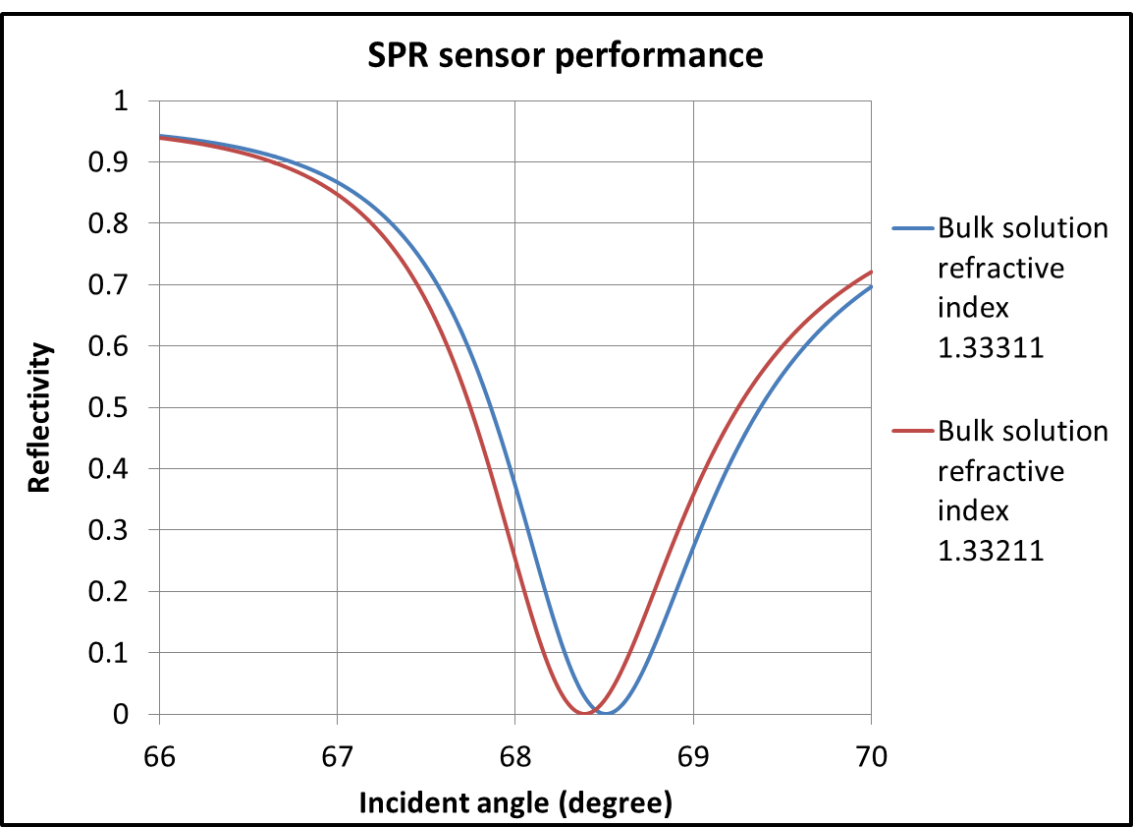

(a) 


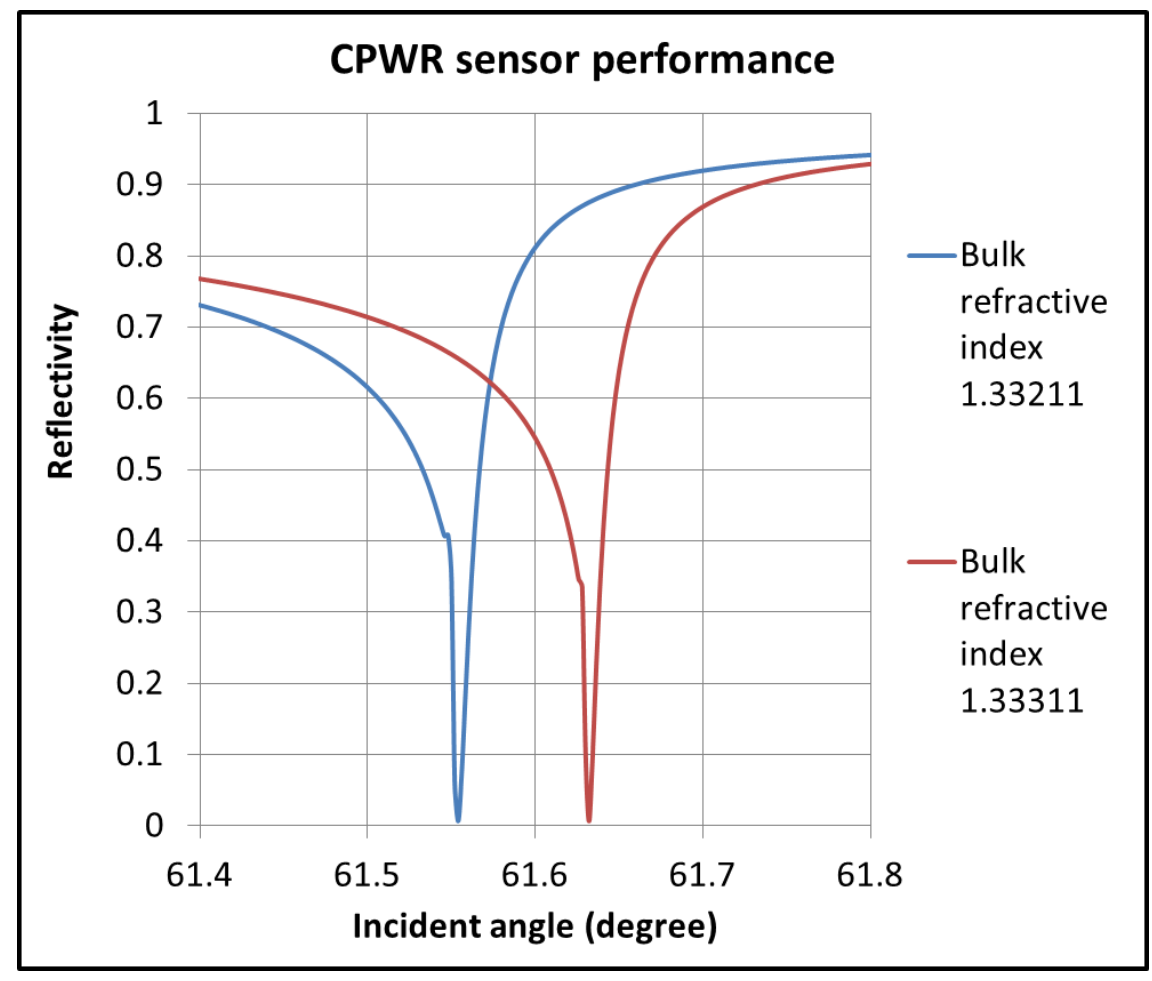

(b)

Figure 7-3: Calculation results of reflectivity against incident angle for (a) SPR and (b) CPWR under a 0.001 refractive index change in bulk solution.

The parameters of the reflectivity profile for the CPWR and the SPR are listed in Table 7-1. The CPWR is 33.3 times better than SPR for the refractive index change in bulk solution.

Table 7-1: Parameters of reflectivity profiles for CPWR and SPR for two bulk solutions having a refractive index change of 0.001 . 


\begin{tabular}{|c|c|c|c|c|c|c|}
\hline Sensor type & resonance & resonance & resonance & FWHM & $\Delta n$ & $\Delta n_{S P R}$ \\
& angle for & angle for & angle shift & $\Delta \theta$ & & \\
& 1.33211 & 1.33311 & & & & \\
\hline CPWR & $61.554^{\circ}$ & $61.632^{\circ}$ & $0.078^{\circ}$ & $0.028^{\circ}$ & 0.00036 & \\
\hline SPR & $68.39^{\circ}$ & $68.51^{\circ}$ & $0.12^{\circ}$ & $1.44^{\circ}$ & 0.012 & \\
\hline
\end{tabular}

Refractive index change from molecular thin film adsorption

For comparing the performance in probing a molecular thin film on the surface of the CPWR and the SPR sensors, the reflectivity curves for an adsorbed layer ( $1 \mathrm{~nm}, 2 \mathrm{~nm}$, and $3 \mathrm{~nm}$ ) having a refractive index 1.46 were simulated. The simulated performances of these two sensors are shown in Figure 7-4 for the (a) SPR and the (b) CPWR. The ratio of the resolutions of these two types of sensors is used as a parameter to show the better performance of CPWR. 


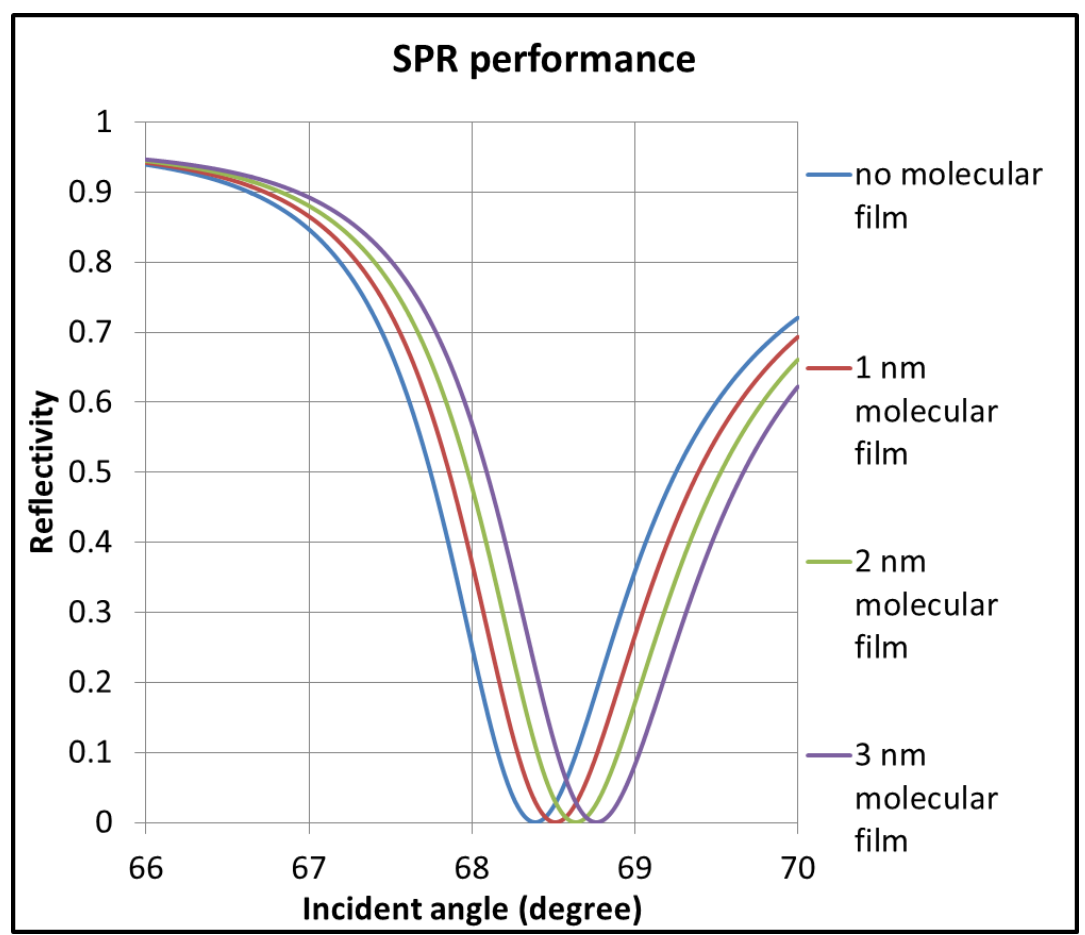

(a)

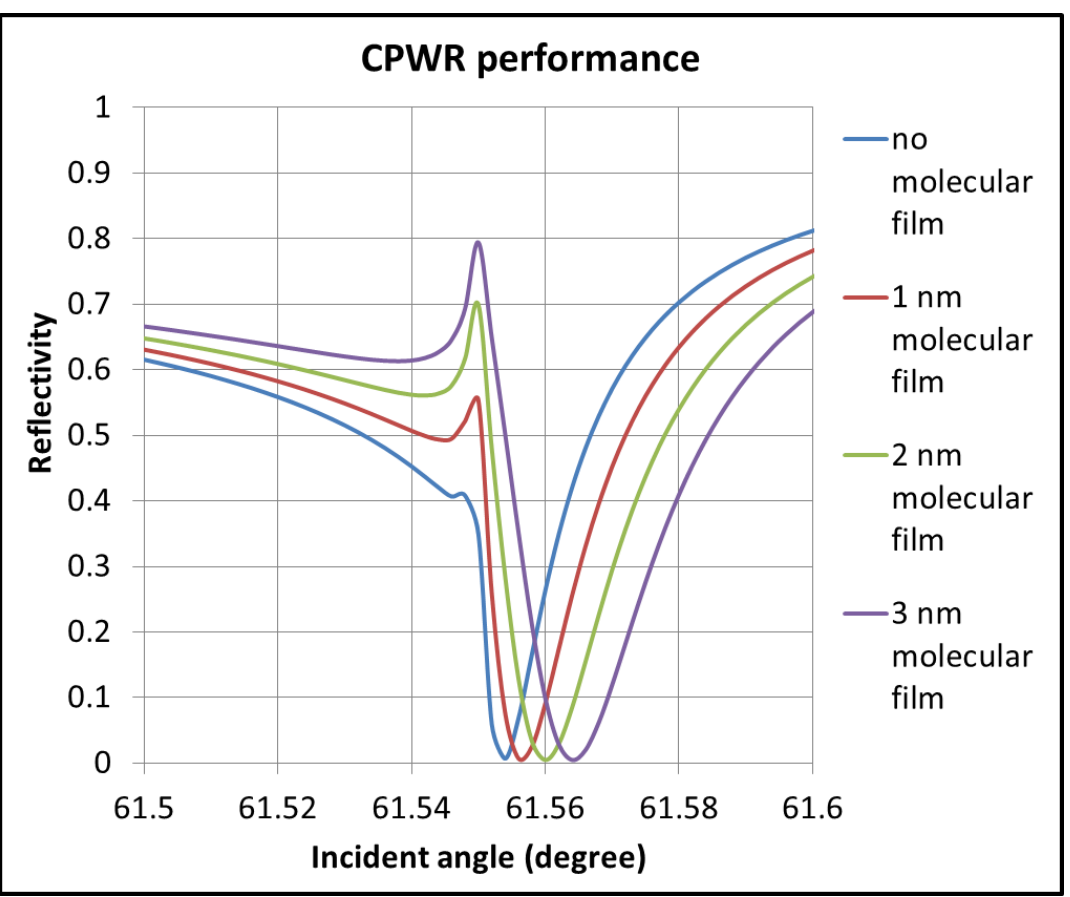

(b)

Figure 7-4: Calculation results of reflectivity curves for $0 \mathrm{~nm}, 1 \mathrm{~nm}, 2 \mathrm{~nm}$ and $3 \mathrm{~nm}$ molecular thin film adsorption on the surface of (a) SPR and (b) CPWR devices. 
The parameters for the reflectivity profiles for both sensors are listed in Table 7-2 for the situation without any molecular film and with a $3 \mathrm{~nm}$ molecular film. As a result, the CPWR sensor has 1.4 times better resolution compared to the SPR sensor.

Table 7-2: Parameters of reflectivity profiles for the CPWR and the SPR for nonmolecular thin film and $3 \mathrm{~nm}$ molecular thin film adsorption.

\begin{tabular}{|l|c|c|c|c|c|}
\hline Sensor & resonance \\
type & angle for 0 nm & resonance & resonance & FWHM $\Delta \theta$ & $\Delta n_{S P R}$ \\
angle for 3 nm & angle shift & & \\
\cline { 1 - 4 } CPWR & $61.554^{\circ}$ & $61.564^{\circ}$ & $0.01^{\circ}$ & $0.028^{\circ}$ & \multirow{2}{*}{1.4} \\
\hline SPR & $68.39^{\circ}$ & $68.76^{\circ}$ & $0.37^{\circ}$ & $1.44^{\circ}$ & \\
\hline
\end{tabular}

In both cases of the refractive index change in bulk solution and a molecular film adsorption, the CPWR has better resolution theoretically compared to the traditional SPR sensor. Based on this configuration (36 nm silver, $5 \mathrm{~nm}$ silicon dioxide and $140 \mathrm{~nm}$ aluminum oxide), the CPWR sensor are fabricated and characterized with the reflectivity curve measurements. 


\section{Fabrication of CPWR}

Microscope slides were used as the substrates. The same examining and cleaning procedures in Appendix 2 were used. A $36 \mathrm{~nm}$ silver film was deposited using the electron beam evaporation under approximately $10^{-6}$ Torr vacuum. Without breaking the vacuum, an additional $5 \mathrm{~nm} \mathrm{SiO} 2$ film was deposited on the surface of the silver film for protection. During the deposition, a layer of a mask was used to cover half of the slide surface to prevent the deposition of silver. The sample was then transferred to an ALD tool, and a $140 \mathrm{~nm}$ of $\mathrm{Al}_{2} \mathrm{O}_{3}$ film was deposited. The reflected intensity of the total reflection from the area without the silver film was used as the reference signal. A picture of the final device is shown in Figure 7-5. The reflectivity curve is generated by taking the ratio of the CPWR sensing signal to the total reflection signal.

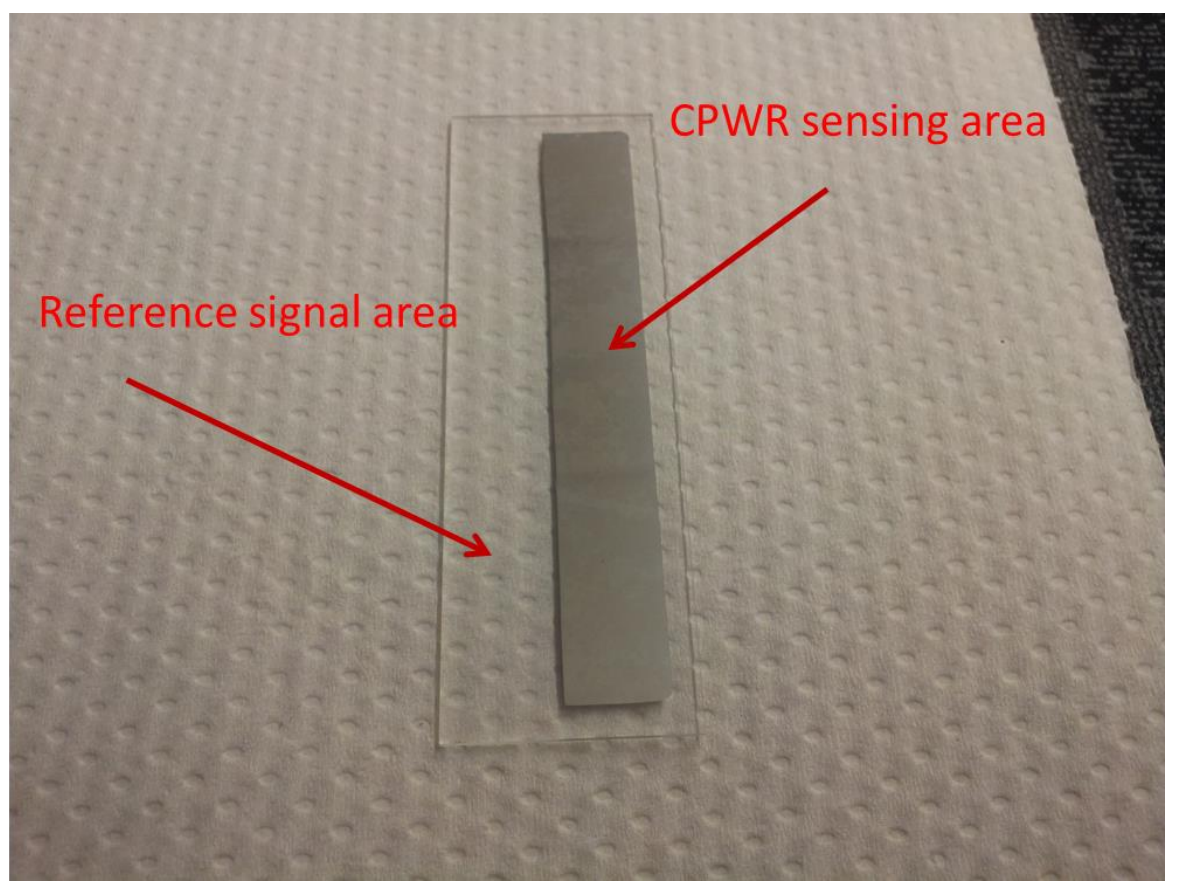


Figure 7-5: Picture of the final CPWR sensor. Half of the area was coated with silver as the CPWR sensing area. The other half area was used to collect the total reflection intensity beyond the critical incident angle.

\section{Sensor Calibration}

After the deposition of each layer, the quality and thickness of this layer were examined. The traditional angular interrogation method was set-up to measure the reflectivity curve, as shown in Figure 7-6. A He-Ne laser was used as the incident beam. A polarizer was used to select the desired polarization. An objective lens was used to focus the beam into a $25 \mu \mathrm{m}$ pin-hole to eliminate higher spatial frequency of the beam. An achromatic doublet was used to collimate the light after the pin-hole. An iris was used to select a desired size of the incident beam. The device was fixed on top of a rotation stage for angle scan measurement. A prism coupler was used to couple the beam into the device to generate the waveguide resonance. The reflected beam intensity was measured by a power meter for each incident angle. The device at different phase of fabrication was examined in air or water solution for characterization. By using a Mathematic program (Appendix 9), simulation result was used to match the measured transmittance and obtain the thickness of each layer. 


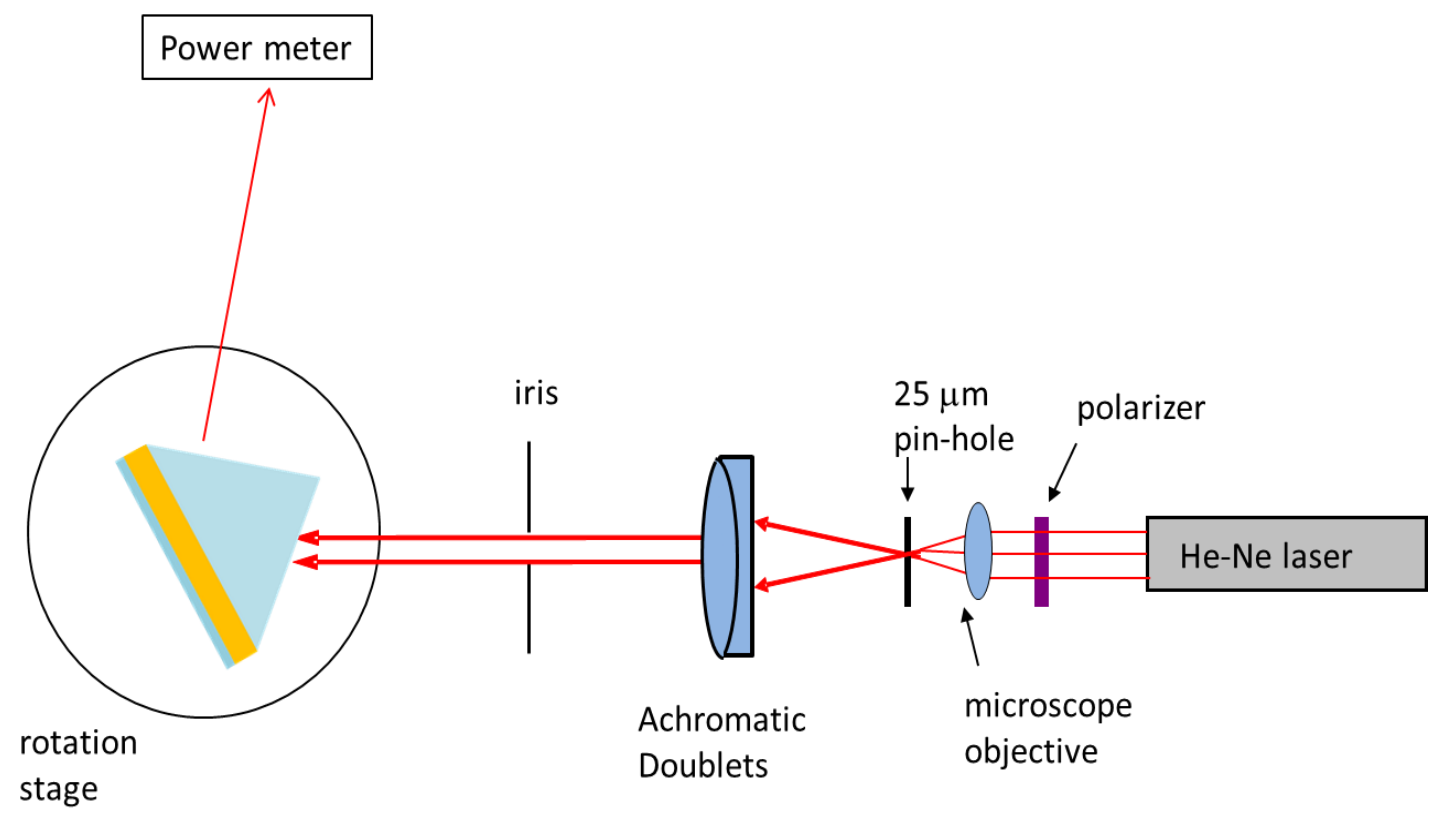

Figure 7-6: Traditional angular interrogation method for the CPWR device performance test.

Thickness of the silver film

The device without an aluminum oxide layer has the conventional SPR structure, and only TM polarized incident light can be used to excite the SP wave, as shown in Figure 7-7. 


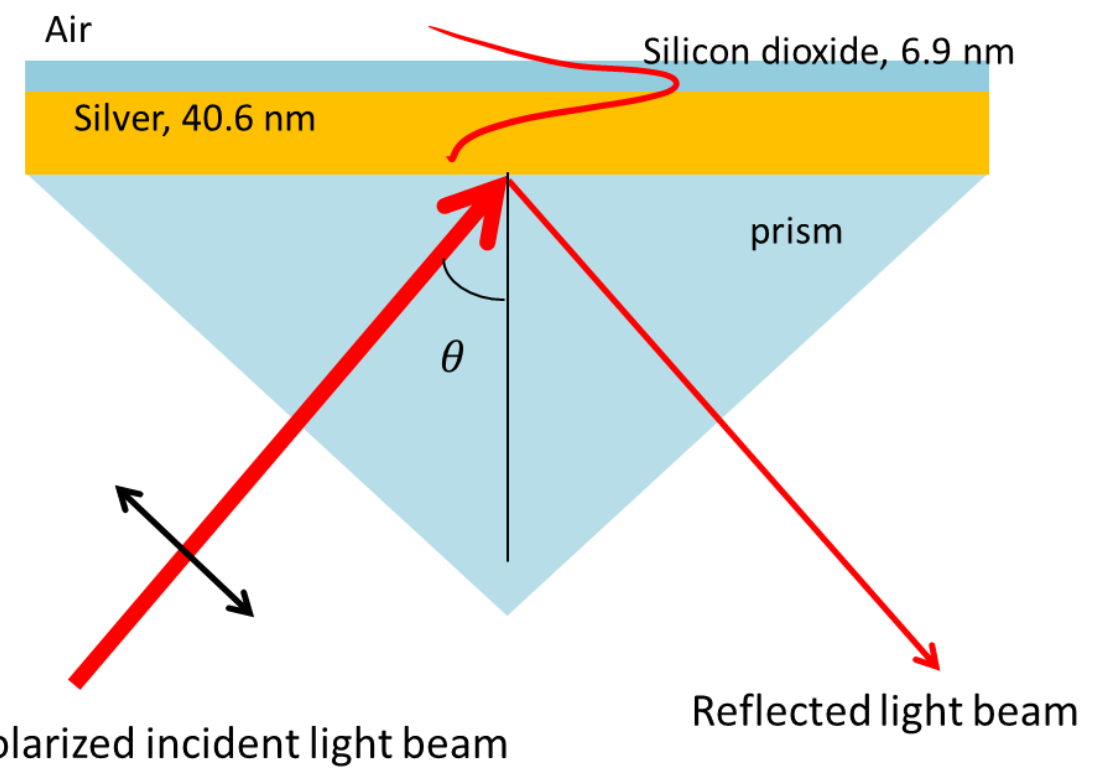

TM polarized incident light beam

Figure 7-7: Thickness examination of silver and silicon dioxide layers. TM polarized incident light is used to excite SP wave.

The reflectivity against the incident angle curve was shown in Figure 7-8 for both the experimental and simulation results. For the simulation, the thickness of silver was $40.6 \mathrm{~nm}$ and the thickness of silicon dioxide was $6.9 \mathrm{~nm}$. The reflectivity at the resonance angle was not as low as the simulation result, as there was a small inaccuracy in the determination of the optical constants and thickness of the films. 


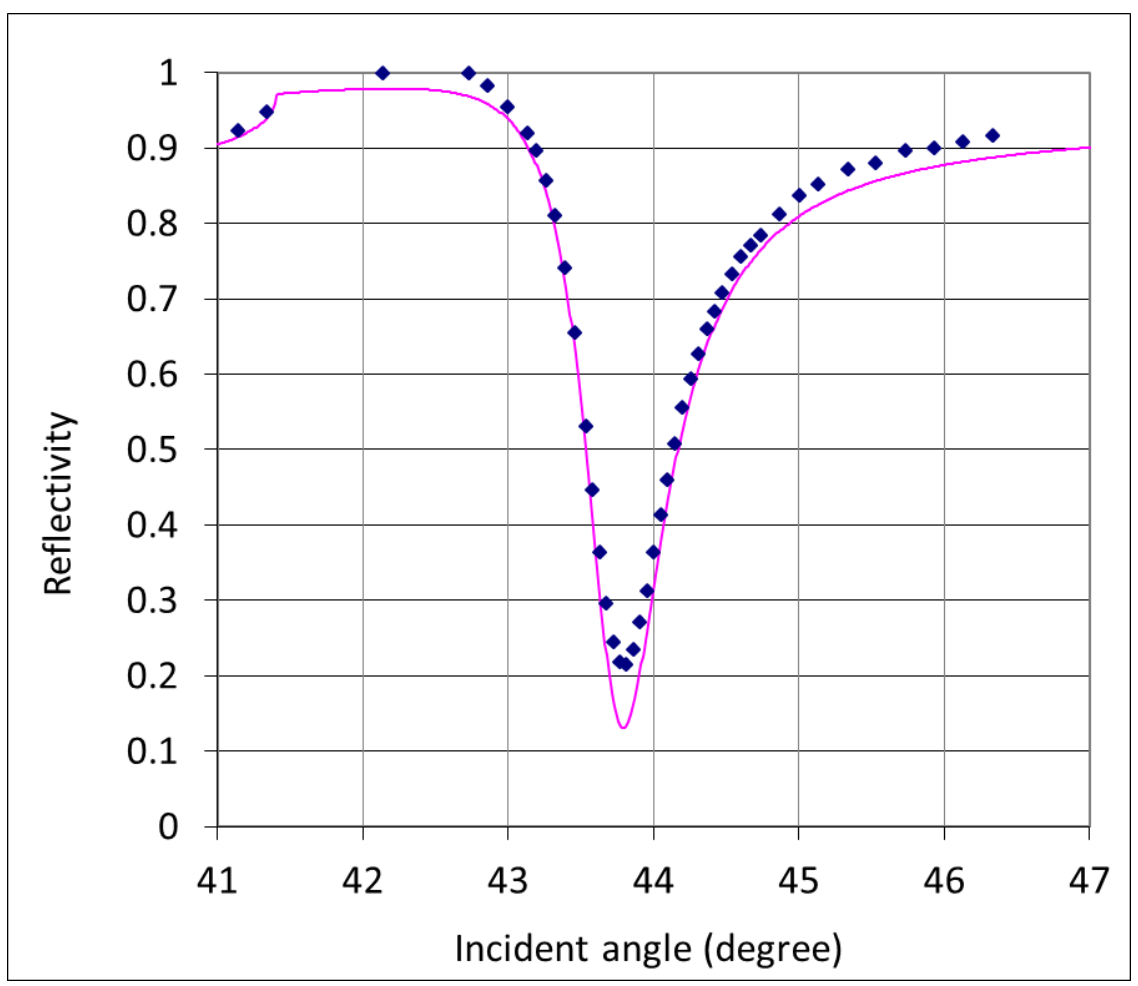

Figure 7-8: The thickness examination for the layer of silver and silicon dioxide. The blue dots are experimental results from the angular interrogation measurement. The pink solid line is simulation result with a $40.6 \mathrm{~nm}$ silver layer and a $6.9 \mathrm{~nm}$ silicon dioxide layer in air.

Thickness of the aluminum oxide layer

After the deposition of the aluminum oxide layer, the reflectivity was measured with the same set-up with a TE polarized incident light, as shown in Figure 7-9. 


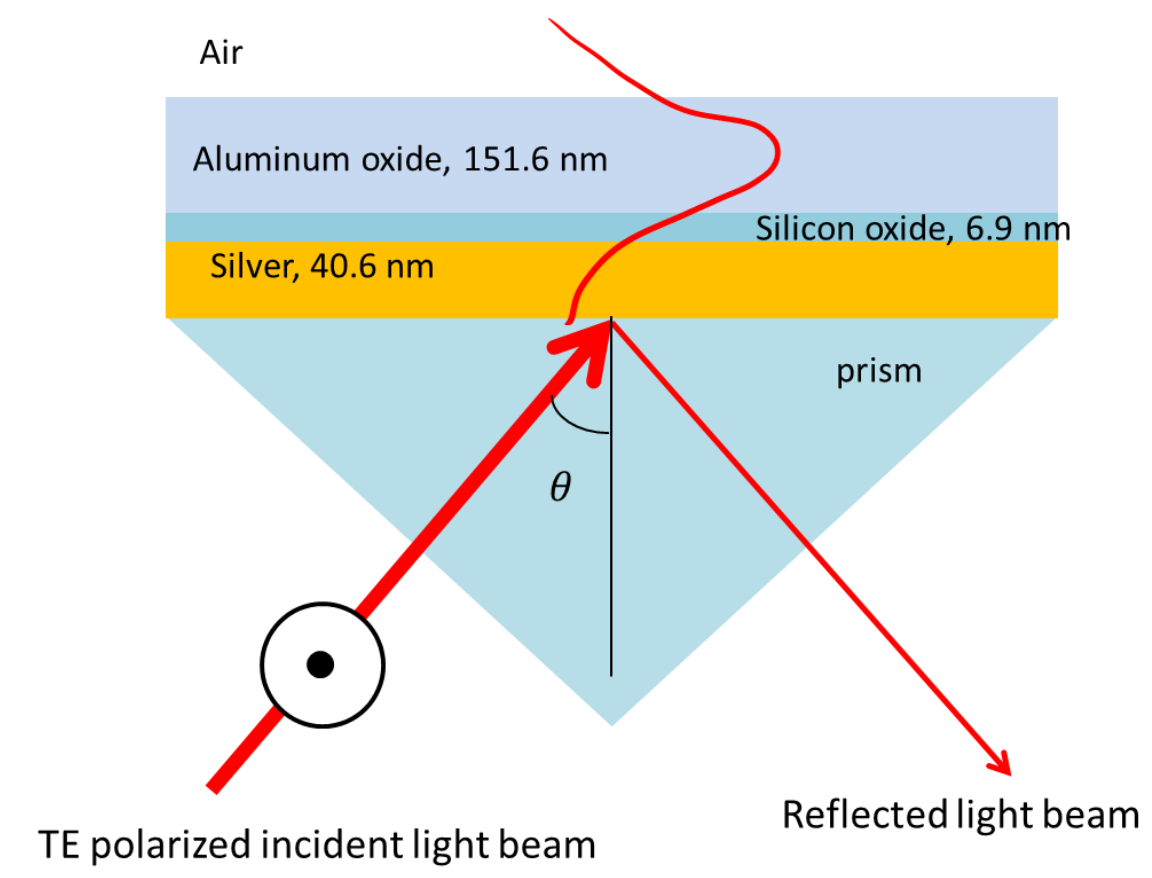

Figure 7-9: Thickness examination of aluminum oxide layer. TE polarized incident light is used to excite waveguide resonance.

The same thicknesses for the silver film and the silicon dioxide film were kept for the reflectivity simulation to examine the thickness of the aluminum oxide layer. The experiment and simulation results were shown in Figure 7-10. Because the configuration of the CPWR was designed to deliver a sharp resonance in the aqueous environment, this particular measurement was collected in air only for the purpose of the device characterization. 


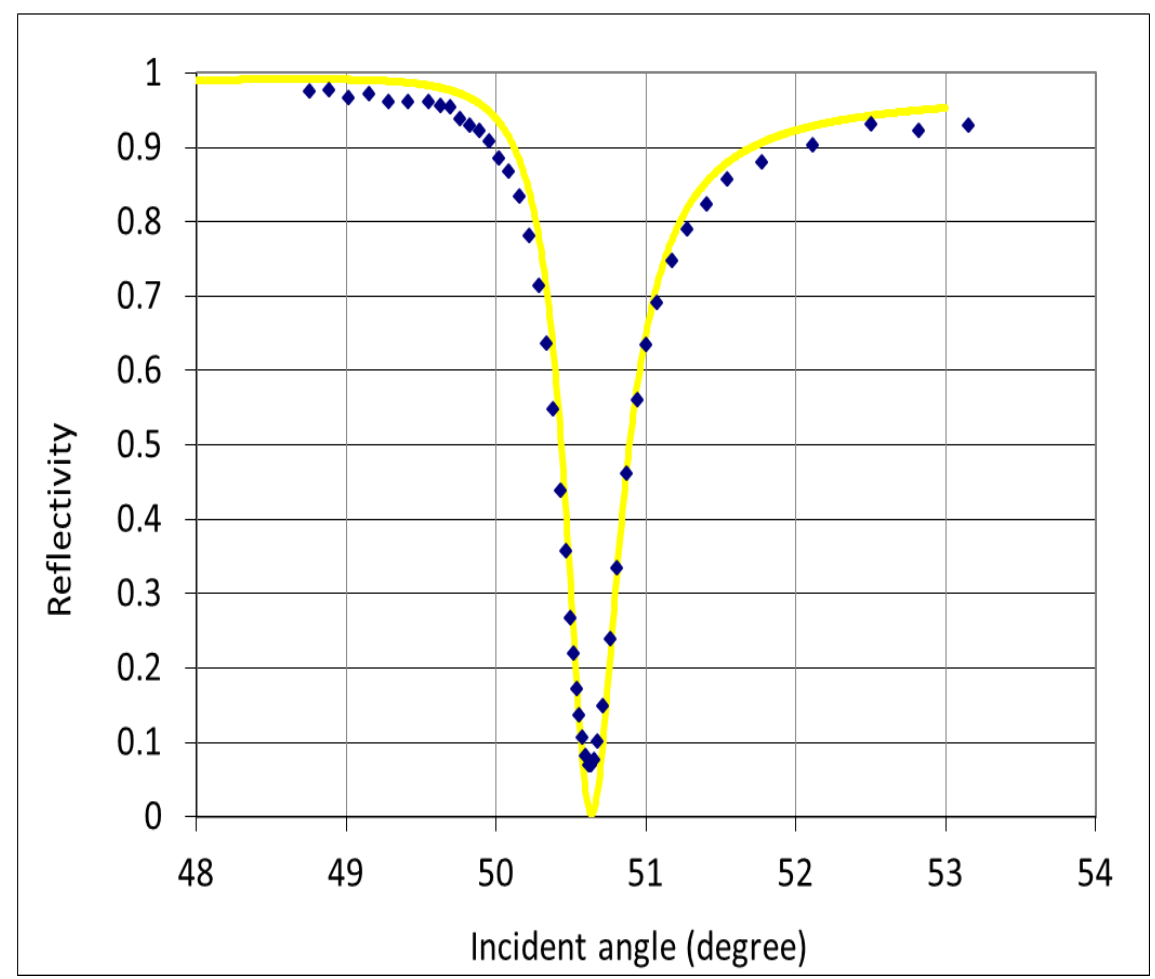

Figure 7-10: Reflectivity against incident angular profile of the CPWR device in air. The blue dots are experimental results from the angular interrogation measurement. And the yellow solid line is the simulation result with a $40.6 \mathrm{~nm}$ silver layer, a 6.9 $\mathrm{nm}$ silicon dioxide layer and a $151.6 \mathrm{~nm}$ aluminum oxide layer in air.

The CPWR device was tested in an aqueous environment. The measured and the simulated reflectivity profiles were shown in Figure 7-11. For the simulation, the calibrated thickness of each layer was kept the same and only the cladding medium was changed from air to water. The angular resonance was much narrower and deeper when compared to the device worked in air. 


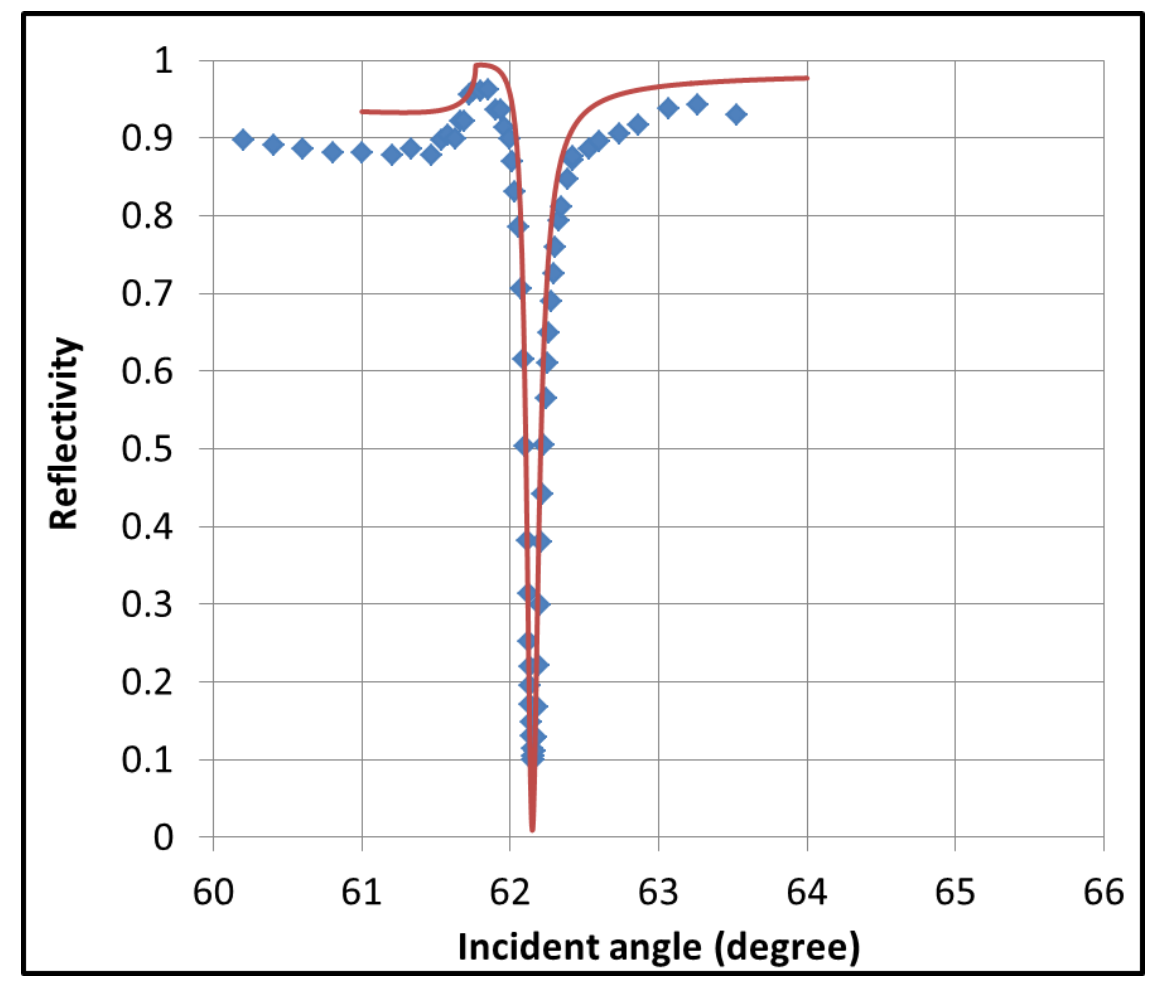

Figure 7-11: Reflectivity against incident angle of CPWR device working in water solution. Blue dots are experimental results from the angular interrogation measurement. And the red solid line is the simulation result with a $40.6 \mathrm{~nm}$ silver layer, a $6.9 \mathrm{~nm}$ silicon dioxide layer and a $151.6 \mathrm{~nm}$ aluminum oxide layer in water.

\section{Conclusions}

We have shown here that CPWR devices need to be properly designed and fabricated in order to materialize a performance improvement over SPR sensors. By using atomic layer deposition to create a high quality optical waveguide over a silver film, we have properly designed and experimentally achieved a CPWR device of 
outstanding performance that surpasses any other CPWR devices reposted so far in literature and reaches superior performance compared to the conventional SPR.

To demonstrate its small resolution, a pump/probe experiment with a realtime fluorescence intensity decay measurement with this CPWR sensor is described in Chapter 8 by using an angle-multiplexed interrogation system. 


\title{
CHAPTER 8
}

\section{APPLICATION OF THE COUPLED PLASMON WAVEGUIDE RESONANCE}

\author{
Introduction
}

The traditional angular interrogation method needs a relatively long time (usually minutes) to scan enough range of incident angles for a surface plasmon resonance (SPR) measurement. Extremely high mechanical and temperature stabilities during the angular scan are needed to resolve small resonance angle shift from a molecular thin film. Experimental set-ups having the real-time detection ability are more desirable to show details of the molecular assembly processes. To achieve the real-time measurement for a molecular thin film studies, an anglemultiplexed system is introduced for the reflectivity measurements. With the small resolution of the CPWR sensors, the kinetics of the fluorescence intensity decay process of a Ru-complex in nano-second scale is examined.

\section{Angle-Multiplexed System}

An angle-multiplexed optical system was built up to have a broadband of incident angles and an ICCD was used to collect the angular spectra information with a nano-second time scale, as shown in Figure 8-1. 


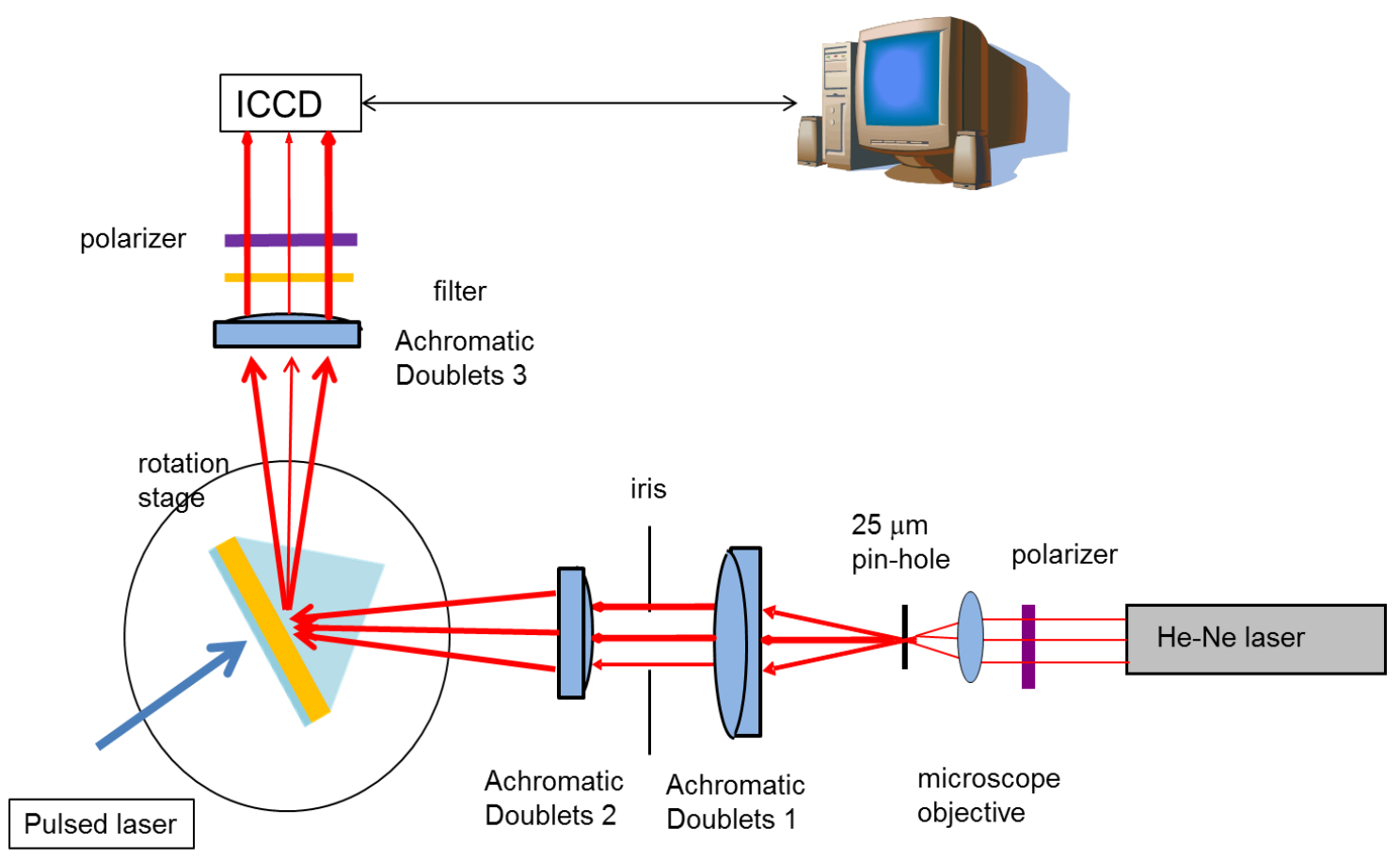

Figure 8-1: Set-up of the angle-multiplexed system.

For the set-up alignment, a He-Ne laser was used as the light source. A polarizer was used to select the polarization. After the polarizer, an objective was used to focus the light into the $25 \mu \mathrm{m}$ pin-hole. Behind the pin-hole an achromatic doublet was used to collimate the beam to achieve uniform distribution of the incident light. An iris was placed after the achromatic doublet to select the size of the incident beam. Since the ICCD detector has an $18 \mathrm{~cm}$ diameter opening, the iris opening was set at the same size. Another achromatic doublet (500 mm effective focal length, Thorlabs AC 254-500-A1) was used to focus the light on the CPWR to excite the waveguide resonance. Another identical achromatic doublet $(500 \mathrm{~mm}$ effective focal length, Thorlabs AC 254-500-A1) was used to collect and collimate the reflected light into the ICCD detector. With the size of the beam $(18 \mathrm{~mm}$ in 
diameter $)$, the number of pixels $(1024 \times 256)$ and the size of a pixel $(26 \mu \mathrm{m} \times 26 \mu \mathrm{m})$ of the ICCD camera, each pixel was related to a particular incident angle. The range of the incident angle was $2.06^{\circ}$ with each pixel corresponding to $0.002^{\circ}$.

After the alignment with the $633 \mathrm{~nm}$ laser beam, the position of the CPWR on the rotation stage was fixed and only the vertical dimension was adjusted to the reference area or the CPWR sensing area. Based on the calibration result from Section 7.4 , the resonant incident angle was at $62.15^{\circ}$ and FWHM was $0.1^{\circ}$. The ICCD camera was set as black-white intensity image mode. When the He-Ne laser beam hit the CPWR sensing area, a dark line would appear in the middle of the image by rotating the rotation stage, as shown in Figure 8-2.

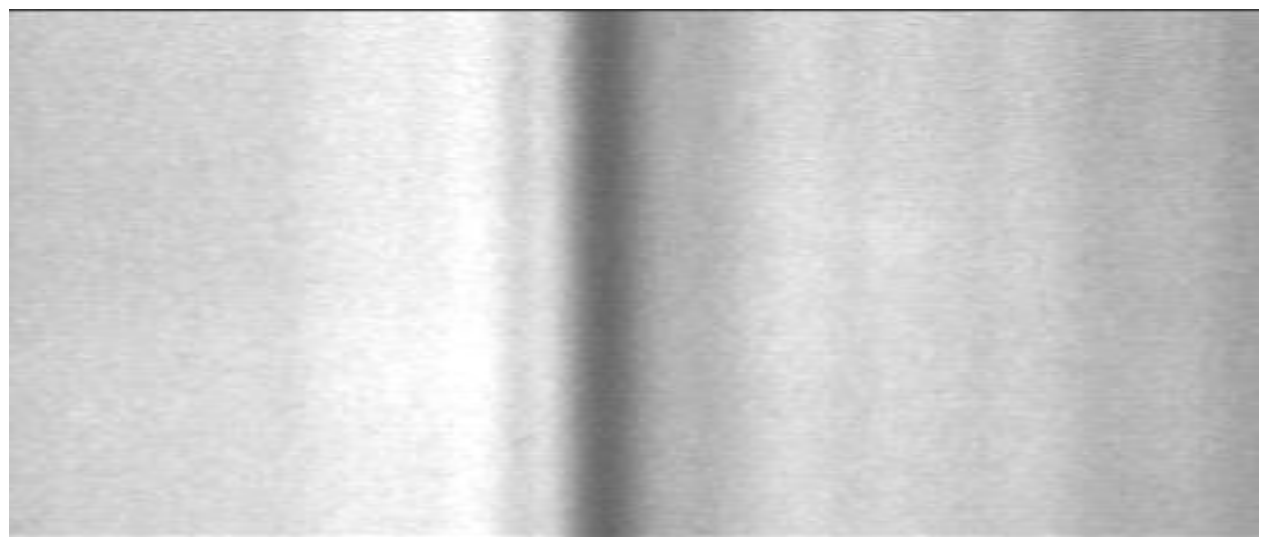

Figure 8-2: Pictures of the ICCD images for the angular resonance when a CPWR sensor working in an aqueous solution.

The same sample calibrated in Section 7.4 was tested with the anglemultiplexed set-up without any excitation laser beam. In Figure 8-3, the raw ICCD 
intensity counts against pixel number was plotted for both the reference signal and the CPWR sensing area.

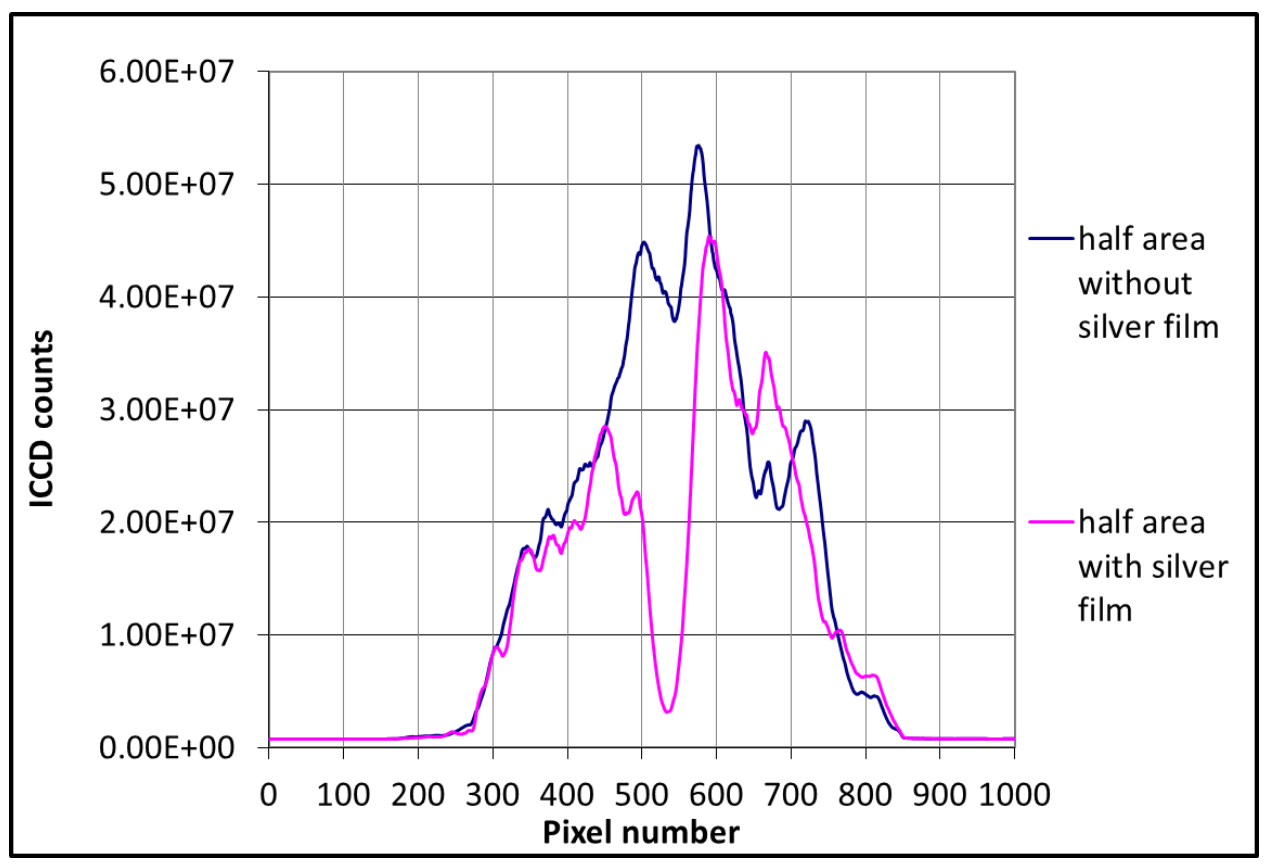

Figure 8-3: Reflected intensity against the pixel number profiles for the CPWR sensing area and the reference area.

In order to calculate the reflectivity for this set-up, Equation 8-1 was used with the dark signal, $I_{D}$ (with $\mathrm{He}-\mathrm{Ne}$ laser off), the reference signal of the total reflection, $I_{T}$, and the CPWR signal, $I_{W}$.

$$
\operatorname{Ref}=\frac{I_{W}-I_{D}}{I_{T}-I_{D}}
$$

The reflectivity curve achieved using the angle-multiplexed approach was compared to the results of the simulation and the traditional scanning angular 
measurements from Section 7.4.2, as shown in Figure 8-4. A consistency was observed among these three curves. With the angle-multiplexed system, the CPWR was capable of the real-time measurements with a small angular resolution.

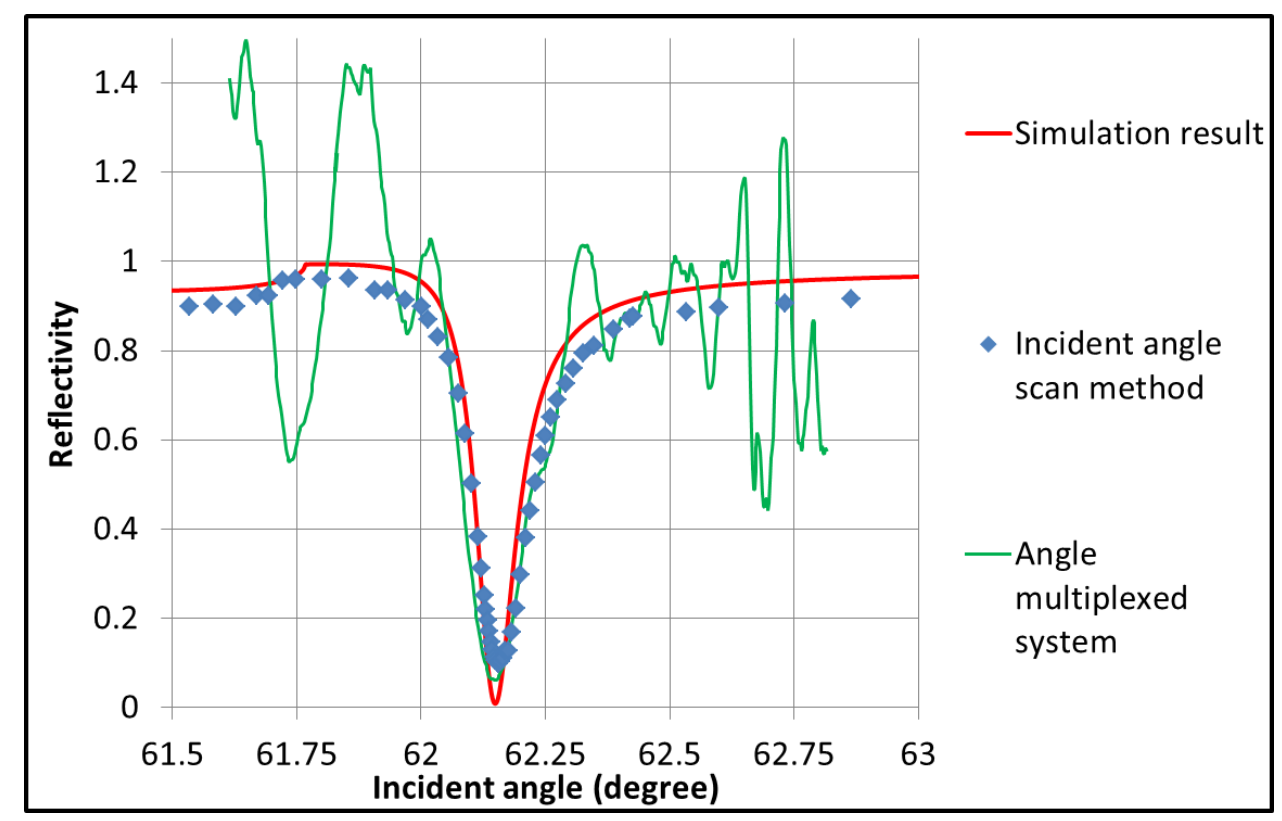

Figure 8-4: Reflectivity curves measured by the angle-multiplexed system compared to the experimental result from the conventional angular interrogation and simulation result for the same CPWR device in an aqueous environment.

\section{Fluorescence Intensity Decay Experiment}

With the angle-multiplexed system, an experiment of fluorescence intensity decay in nano-second scale is used to show the small resolution of the CPWR sensor. The molecules adsorption process is monitored by the He-Ne laser. The fluorescence 
intensities at specific time delay after the pulsed laser excitation are measured [216218].

\section{Experiment preparation}

Because the aluminum oxide film was hydrophobic, to immobilize molecules a $1 \mathrm{~nm}$ layer of PEG silane (2-[methoxy- (polyethyleneoxy)propyl]trimethoxysilane) was functionalized on the $\mathrm{Al}_{2} \mathrm{O}_{3}$ surface by the molecular vapor deposition.

The fluorescent molecule $\mathrm{Ru}(\mathrm{bpy})_{2}$ (mcbpy-O-Su-ester) $\left(\mathrm{PF}_{6}\right)_{2}$ (Ru-complex, molecular weight $1,014.66 \mathrm{~g} / \mathrm{mol}$, formula is $\mathrm{C}_{36} \mathrm{H}_{29} \mathrm{~F}_{12} \mathrm{~N}_{7} \mathrm{O}_{4} \mathrm{P}_{2} \mathrm{Ru}$ ) is used. Its chemical structure is shown in Figure 8-5. The excitation wavelength is $458 \mathrm{~nm}$. The fluorescent emission wavelength is $628 \mathrm{~nm}$, which is close to the wavelength of the He-Ne laser. The lifetime for the fluorescence is approximately $500 \mathrm{~ns}$. The Rucomplex was mixed with anhydrous acetonitrile (99.8\%) to make a stock solution. For the experiment, the stock solution was diluted to a concentration of $8 \mu \mathrm{M}$ with 7 $\mathrm{mM}$ phosphate buffer of and $1 \mathrm{mM} \mathrm{NaCl}$. The $\mathrm{pH}$ value was 7.2. 


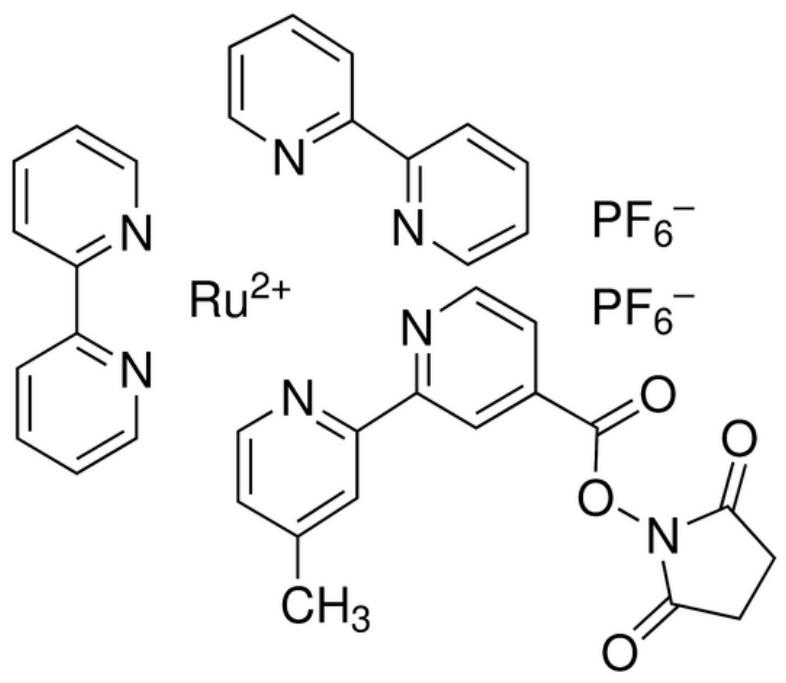

Figure 8-5: Chemical expression of Ru-complex.

To excite the fluorescence, a pulsed laser (Continuum, Panther EX OPO with doubler and Surelite SL I-20) was used from the backside of the flow cell, as shown in Figure 8-6. The excitation beam was aligned with the He-Ne laser beam. The molecules located inside of this excitation beam area were excited, and the fluorescence, having the same wavelength as the He-Ne laser beam, was coupled out through the reflection light path into the ICCD camera. Another polarizer was used in the spectra collection light path, and A $550 \mathrm{~nm}$ long-wave pass filter (Thor Laboratories) was placed in front of the ICCD to eliminate any scattered light of the pulsed laser beam. 


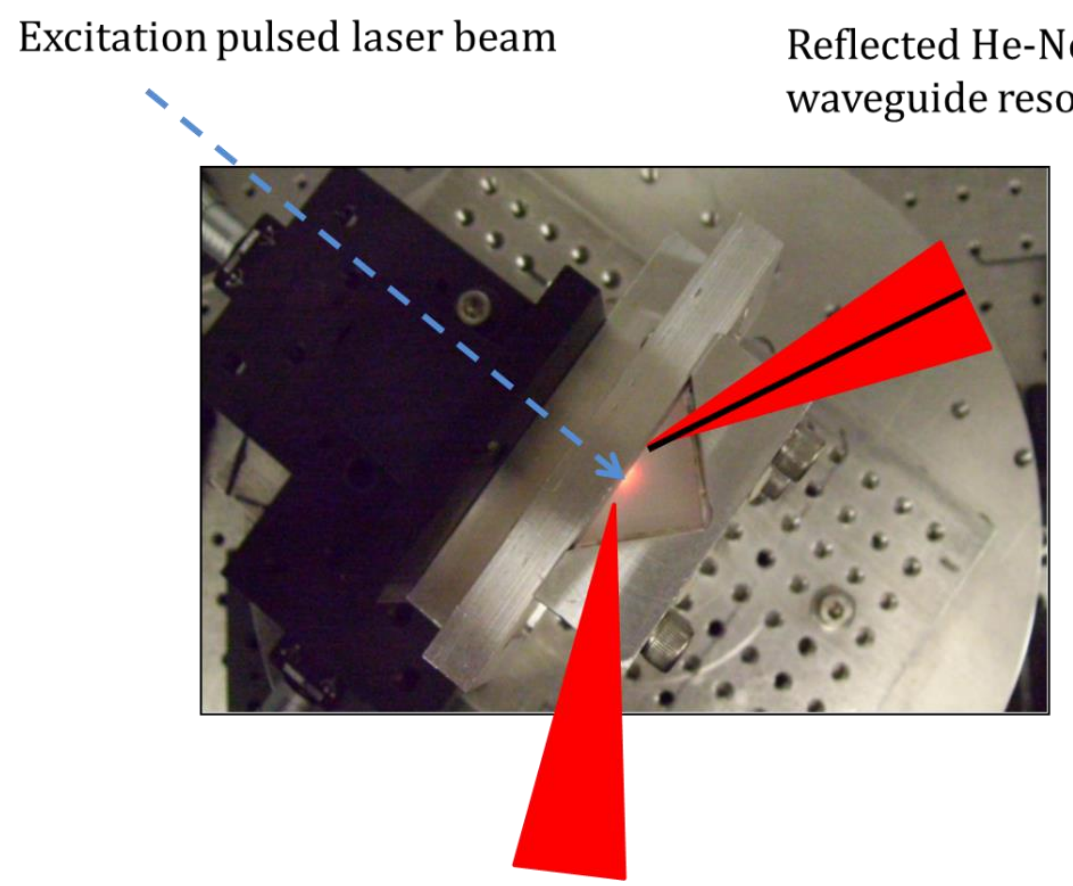

Incident He-Ne laser beam

Figure 8-6: Top view of the flow cell mounted on a rotation stage. The blue arrow represents the excitation pulsed laser beam. This beam is aligned with the He- $\mathrm{Ne}$ laser beam at the same spot on the CPWR sensor.

\section{Reference signals collection}

The He-Ne- laser was on and the buffer solution was injected into the flow cell. A reflected intensity profile from the reference signal area was collected for the total reflection intensity. Then the device was moved vertically to the CPWR sensing area for a reflectivity curve measurement. The set-up for the ICCD of this experiment were 1000 frames of accumulation with no gain and gated mode with $100 \mathrm{~ns}$ exposure time for each frame. 
A group of control signals were collected to show that there was no fluorescence signal from the CPWR device under the excitation laser beam. The HeNe laser was turned off and the tunable pulsed laser was turned on. The pulsed laser beam was centered at $458 \mathrm{~nm}$ with the pulse having approximately $5 \mathrm{~ns}$ duration. The pulses were set with a repetition rate at $20 \mathrm{~Hz}$. An external trigger from the pulsed laser was sent to the ICCD camera. The angular spectrum was taken at the delay of $10 \mathrm{~ns}, 120 \mathrm{~ns}, 230 \mathrm{~ns}, 340 \mathrm{~ns}, 450 \mathrm{~ns}$, and $1000 \mathrm{~ns}$ after the external trigger. The ICCD exposure parameters were set to a $10 \mathrm{~ns}$ exposure time, 15,000 frames accumulation, and a gain of 50. No fluorescence was detected from the CPWR sensor in the buffer solution.

\section{Ru-complex adsorption}

For characterization of the adsorption process of the Ru-complex on the waveguide surface, the He-Ne laser was turned on and the pulsed laser was turned off. The exposure time for the ICCD for this step was set as 1000 frames of accumulation with no gain and $10 \mathrm{~ns}$ gated mode. A reference signal was collected first. The Ru-complex solution was slowly injected into the flow cell. When no further resonance angle shift was observed, the buffer solution was injected into the flow cell to remove non adsorbed Ru-complex molecules. This step was used to prevent the floating ones from being excited by the pulsed laser beam and affecting the signal from the surface immobilized ones. Angular reflectivity profile was measured at different time until a new equilibrium was achieved. Figure 8-7 shows the angular reflectivity traces using the $\mathrm{He}-\mathrm{Ne}$ laser for the pure buffer solution, 6 
minutes after the Ru-complex sample injection, and 4 minutes after the flow cell flushing.

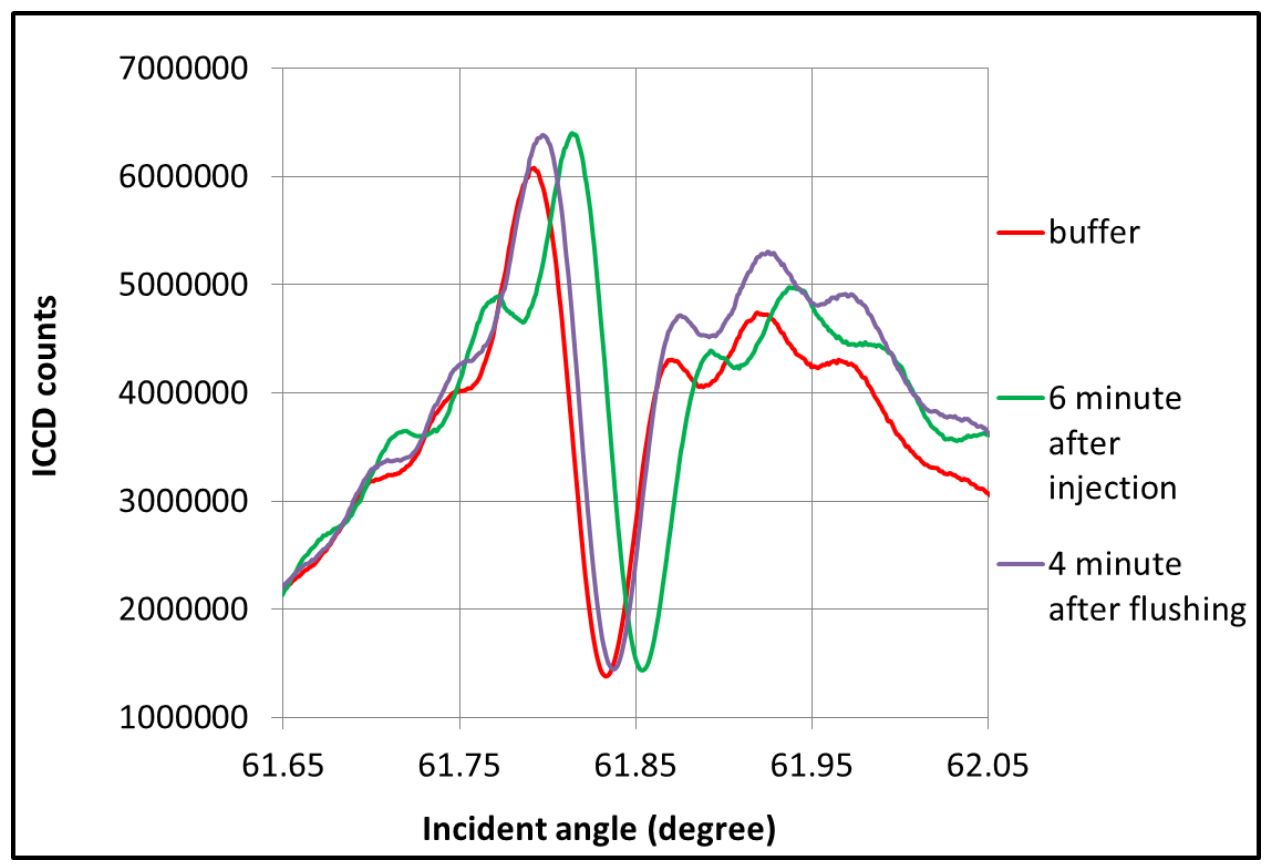

Figure 8-7: Resonance angle shift for the Ru-complex molecule adsorption monitoring.

The data showed that after the adsorption process reached the equilibrium, the angle of resonance increased by $0.021^{\circ}$. When the flow cell was flushed with the buffer solution, the resonant angle shifted backward by $0.018^{\circ}$, indicating desorption of the loosely bound molecules. By comparing the final angular spectrum to the one of pure buffer solution, resonant angle had a small but consistent increase of $0.003^{\circ}$, indicating that $\mathrm{Ru}$-molecules were indeed adsorbed on the surface of the device. 


\section{Kinetics of the fluorescent decay}

After a molecular thin film of Ru-complex was immobilized on the surface of the CPWR device, the He-Ne laser was turned off and the pulsed laser was turned on to excite these Ru-complex molecules. The same set-up for the pulsed laser and delay measurement for the control group signals were used for Ru-complex molecules.

The fluorescence intensity at different time delay was shown in Figure 8-8 for TE polarization. Upon excitation, the adsorbed Ru-complex molecules radiated in every direction, and the light that was partially transmitted through the device and the prism was captured. However, the light emitted at the resonant angle was substantially coupled into the waveguide mode which created a dip in the trace of the angular spectrum, as shown in Figure 8-8. With increasing time delays, the fluorescence emission intensity surrounding this dip was decreased. This conclusion was supported by the fact that the resonance angle location of the dip was the same as the resonant angle, shown in Figure 8-7 when the system was at the equilibrium after the buffer flushing. The reflectivity curve was shifted evenly to show the difference. 


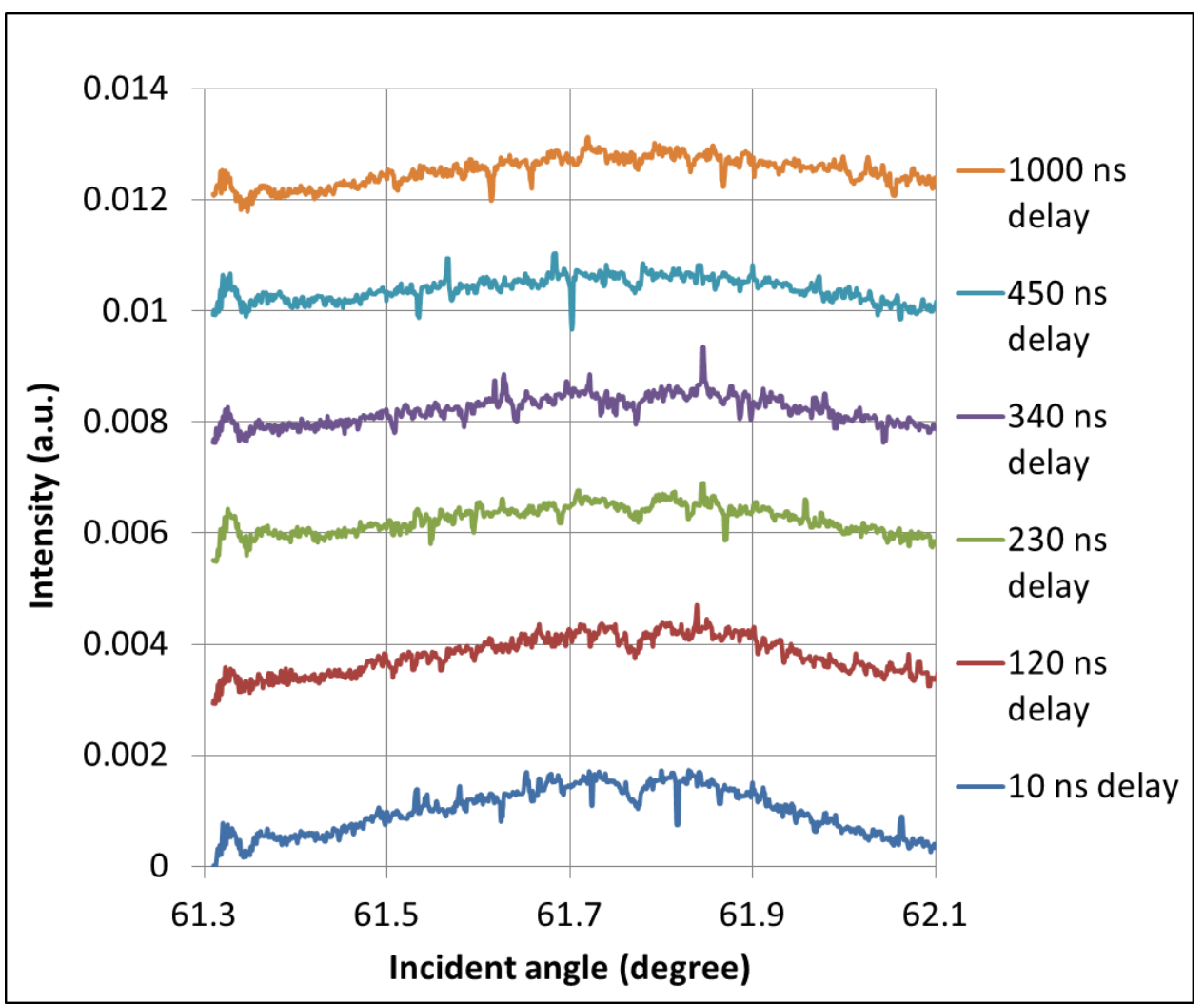

Figure 8-8: Fluorescence intensity profiles for TE polarized incident light at different time delay.

The height of the dips for different time delay was used to calculate the fluorescence lifetime of the Ru-complex film. The result was shown in Figure 8-9 with an exponential fitting. The emission lifetime was 439 ns based on the fitting result, which was consistent with the expected value of $500 \mathrm{~ns}$ (Sigma Aldrich). 


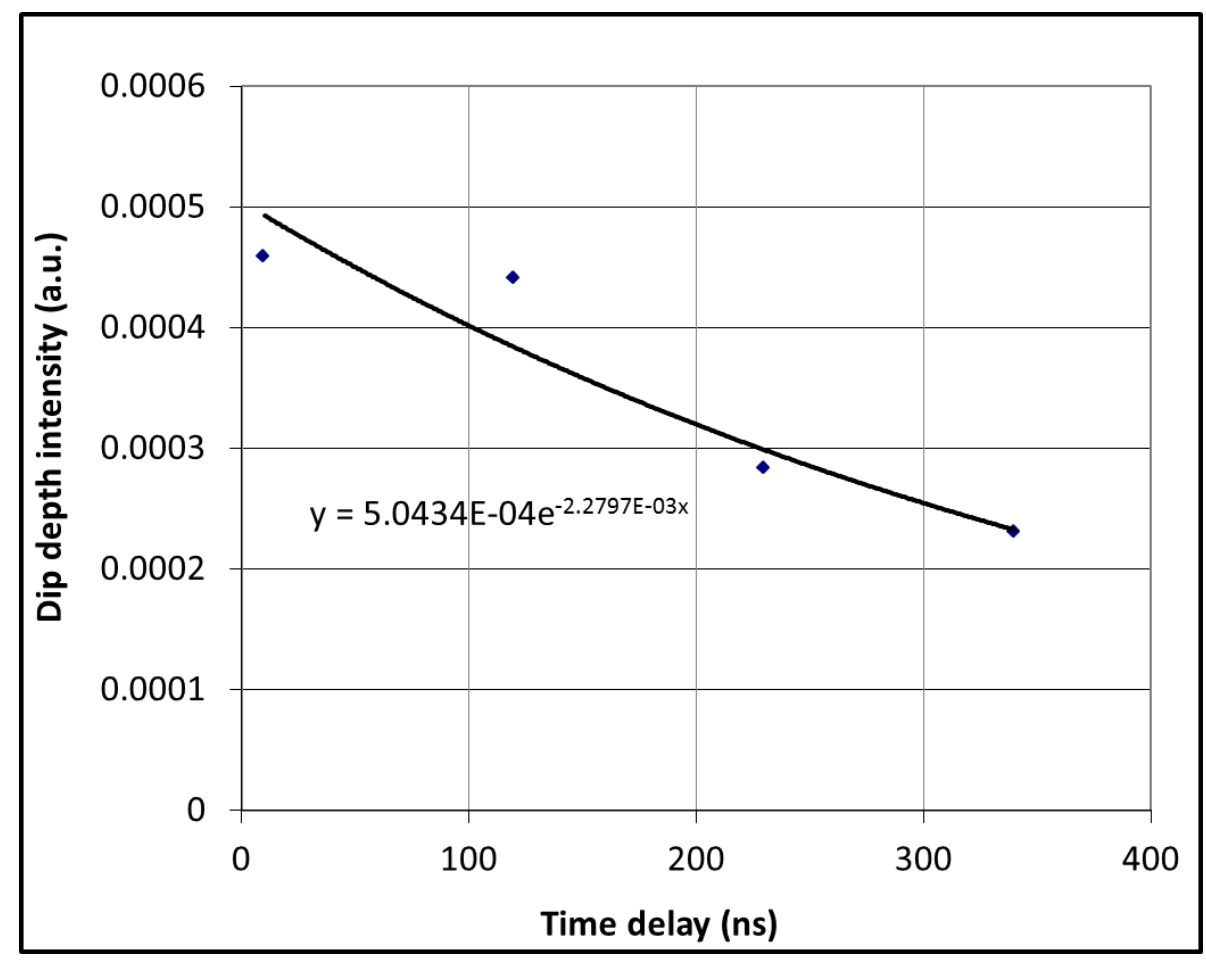

Figure 8-9: Exponential fitting for the time constant of Ru-complex fluorescence decay by using the height of the dip from each time delay.

The same experiments with TM polarized incident light were carried out and the reflected intensity curves were shown in Figure 8-10. As expected, no strong coupling occurred in these measurements because the waveguide layer could not support a TM polarized guided mode. The reflectivity curve was shifted evenly for each time delay. 


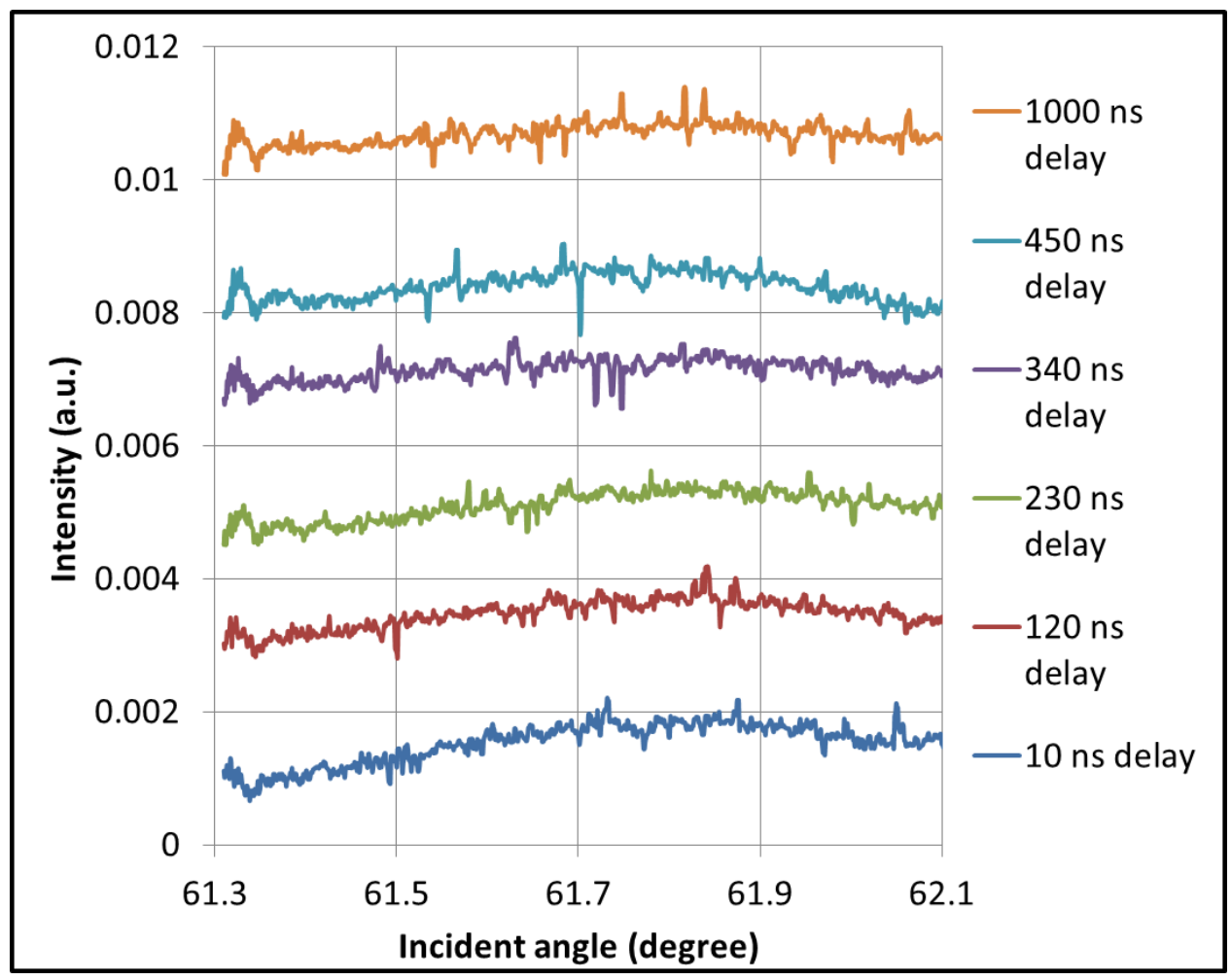

Figure 8-10: Fluorescence intensity profiles for TM polarization at different time delay.

\section{Conclusions}

A small angular resolution CPWR sensor combined with an anglemultiplexed experiment set-up was used to test fluorescence intensity decay process in real-time with nano-second scale. With the ability of detection of a submonolayer in real-time, this combination can be used to detect other processes involving a low surface concentration of molecules and fast evolvements. 


\title{
CHAPTER 9
}

\section{SUMMARY AND OUTLOOKS}

\begin{abstract}
Summary
Two novel optical analytical techniques based on single-mode optical waveguides were designed, fabricated and experimentally characterized for molecular assembly studies.

The electro-active single-mode integrated optical waveguide (EA-SM-IOW) was achieved by creating a transparent and conductive ultra-thin indium tin oxide (ITO) film over a SM-IOW. A precise sputtering deposition process and a combination of different annealing procedures were used to optimize the optical and electrical properties of the ITO electrode. The spectroscopic features of the ultra-thin ITO films under three types of potential modulations were thoroughly investigated. As the results have shown, the imaginary component of the ITO refractive index was the major contributor to the dependence on the applied potential of the out-coupled optical intensity propagating along the EASM-IOW. As such optical signal represented baselines for spectroelectrochemical experiments investigating surface adsorbed redox species, a new analytical strategy was developed for retrieving accurate electrochemical information under alternating
\end{abstract}


current potential modulation in impedance measurements. Experimental demonstration have shown that the EA-SM-IOW platform could detect and characterize surface coverage of redox active species as low as $10^{-15} \mathrm{~mol} / \mathrm{cm}^{2}$. With the extremely high sensitivity of the novel optical analytical platform and the accurate methodology for retrieving electrochemical information, optical impedance spectroscopy measurements were applied to study electron transfer processes of the cytochrome c proteins directly adsorbed to the ITO electrode under different surface concentration and different ionic strength situations. High surface density of redox species and electrolyte solution with strong ionic strength have shown to slower the electron transfer process between the cytochrome c proteins and the ITO electrode surfaces.

A coupled plasmon waveguide resonance (CPWR) platform was developed by creating a high quality single mode waveguide based of a dielectric aluminum oxide film on top of a thin layer of silver. The experimental characterization has proven a deep and narrow resonance for our novel device, as predicted theoretically and superior to any other reports described so far in the literatures. Experimental tests for changes in the refractive index of a bulk solution and the growth of a molecular adsorbate were examined for conventional SPR and CPWR under their best theoretical configurations. CPWR has shown a smaller and therefore a better angular resolution for both cases. An angle-multiplexed system was built up to excite the CPWR with a broadband incident angle. At the resonance angle, a dark line corresponding to the resonance angle was observed. The fluorescent from a surface immobilized Ru-complex molecular assembly was tested with a pulse laser 
excitation source. The kinetics of the fluorescent decay was captured by using an ICCD camera with a nano-second time resolution.

\section{Outlooks}

With these two sensitive optical analytical tools, molecular assemblies at low surface densities can be studied for fundamental research, and novel sensors can be implemented to increase sensing limits.

Based on the optimized optical and electrical properties of the ITO film, EASM-IOW platform creates several opportunities for electrochemical sensing applications.

Sensors for the detection of superoxide radical and characterization of their concentrations influence on antioxidants have attracted great interests. Increasing evident show that superoxide is related to many human diseases. This radical has shown strong activity to proteins, DNA, and lipid layer cell compartments, so it has a short life time typically from millisecond to second. It is a byproduct of a few enzyme reactions, so the physiological concentration is quite low, in the order of $10^{-11} \mathrm{M}$. One of the analytical tools is based on the reduction of the cytochrome c proteins by the superoxide radical. A potential can apply to the electrode to oxidize the cytochrome c proteins, which generates a current proportional to the concentration of the superoxide. The challenge for these sensors has been the low concentration of cytochrome c proteins that can be immobilized on the electrode surface for the current signal generation. Different types of mediator layers have 
been tested to enhance the detection signals [3-6]. With the new OIS methodology and EA-SM-IOW platform, the optical probing strategy described here can be applied to detect superoxide radical in low concentration to overcome those challenges.

The EA-SM-IOW technology can also be applied towards the development of novel optoelectronic materials and devices, as in solar cells[219-221], organic light emitting diodes (OLED) [222] and organic substrates [223]. Taking the solar energy application as an example, there is a strong need for development of novel materials, as the wide band gap of conventional materials is efficient to collect only a small part of the sun light spectrum (in the ultra violet band). Novel synthesized materials are expected to be designed for enhancing optical absorption in the visible and infrared bands for better usage of the solar energy. Understanding the photon induced electron transfer process in a donor-accepter linked system under welldefined conditions is essential for improving devices performances and efficiency. Improving the charge generation efficiency, slowing the recombination processes and increasing the collection efficiency are major tasks of research for these organic materials [224]. The EA-SM-IOW platform can be a useful tool to study the electron transfer process in those inhomogeneous organic materials. 


\section{REFERENCES}

1. Ataka, K. and J. Heberle, Bioenergetics at the gold surface: SEIRAS probes photosynthetic and respiratory reactions at the monolayer level. Biochemical Society transactions, 2008. 36(Pt 5): p. 986-91.

2. Robert H. Callender, R.B.D., Rudolf Gilmanshin, and William H. Woodruff, Fast events in protein folding the time evolution of primary process. Annual review of physical chemistry, 1998. 49: p. 173-202.

3. Jian Chen, U.W., Fred Lisdat, Bixia Ge, Frieder W. Scheller, Superoxide sensor based on hemin modified electrode. sensors and Actuators B: Chemical, 2000. 70: p. 6.

4. Moritz K. Beissenhirtz, F.W.S., and Fred Lisdat, A Superoxide Sensor Based on a Multilayer Cytochrome c Electrode. Analytical chemistry, 2004. 76(16): p. 7.

5. K. Vengatajalabathy Gobi, F.M., Efficient mediatorless superoxide sensors using cytochrome c modified electrodes surface nano-organization for selectrivity and controlled peroxidase activity. Journal of Electroanalytical Chemistry, 2000. 484: p. 10.

6. Hensley, K. and R.A. Floyd, Reactive oxygen species and protein oxidation in aging: a look back, a look ahead. Archives of Biochemistry and Biophysics, 2002. 397(2): p. 377-83. 
7. Li, Y., et al., Highly sensitive fluorescent immunosensor for detection of influenza virus based on Ag autocatalysis. Biosensors \& bioelectronics, 2013. 54C: p. 358-364.

8. Tan, Y., et al., Proximity-dependent protein detection based on enzymeassisted fluorescence signal amplification. Biosensors \& bioelectronics, 2014. 51C: p. $255-260$.

9. Hye Jin Lee, A.W.W., and Robert M. Corn, Creating Advanced Multifunctional Biosensors with Surface Enzymatic Transformations. Langmuir, 2006. 6(22): p. $5241-5250$.

10. Turzhitsky, V., et al., Spectroscopy of scattered light for the characterization of micro and nanoscale objects in biology and medicine. Applied Spectroscopy, 2014. 68(2): p. 133-54.

11. Natelson, D., Y. Li, and J.B. Herzog, Nanogap structures: combining enhanced Raman spectroscopy and electronic transport. Physical chemistry chemical physics : PCCP, 2013. 15(15): p. 5262-75.

12. Park, J., et al., An approach toward SNP detection by modulating the fluorescence of DNA-templated silver nanoclusters. Biosensors and Bioelectronics, 2013.

13. Aoki, P.H., et al., SERS Mapping in Langmuir-Blodgett Films and SingleMolecule Detection. Applied Spectroscopy, 2013.67(5): p. 563-9. 
14. Chirlmin Joo, H.B., Yuji Ishitsuka, Chittanon Buranachai, and Taekjip Ha, Advances in single-molecule fluorescence methods for molecular biology. Annual review of biochemistry, 2008. 77: p. 51-76.

15. Nirschl, M., F. Reuter, and J. Vörös, Review of Transducer Principles for LabelFree Biomolecular Interaction Analysis. Biosensors, 2011. 1(4): p. 70-92.

16. Baird, C.L. and D.G. Myszka, Current and emerging commercial optical biosensors. Journal of molecular recognition : JMR, 2001. 14(5): p. 261-8.

17. Saen-Oon, S., M.F. Lucas, and V. Guallar, Electron transfer in proteins: theory, applications and future perspectives. Physical chemistry chemical physics : PCCP, 2013. 15(37): p. 15271-85.

18. Dronov, R., Multi-component protein films by layer-by-layer assembly and electron transfer. 2007.

19. Dorothee Grieshaber, R.M., Janos Voros and Erik Reimhult, Electrochemical Biosensors - Sensor Principles and Architectures. Sensors, 2008. 8: p. 1400-1458.

20. Fraser A. Armstrong, G.S.W., Recent developments in faradaic bioelectrochemistry. Electrochemica acta, 2000. 45: p. 23.

21. Katsumi Niki, J.R.S., Emanuel Margoliash, Intermolecular biological electron transfer an electrochemical approach. bioelectrochemistry, 2002. 55: p. 37-40.

22. Armstrong, F.A., H.A. Heering, and J. Hirst, Reaction of complex metalloproteins studied by protein-film voltammetry. Chemical Society Reviews, 1997. 26(3): p. 169. 
23. Bertrand, C.L.a.P., Direct Electrochemistry of Redox Enzymes as a Tool for Mechanistic Studies. Chemical Reviews, 2008. 109: p. 2379-2438.

24. A.V. Patil, J.J.D., Molecular scale bioelectrochemistry. Coordination chemistry reviews, 2011. 255: p. 11.

25. Stefan Gritsch, P.N., Fritz Ja"hnig, and Erich Sackmann, Impedance Spectroscopy of Porin and Gramicidin Pores reconstituted into supported lipid bilayers on indium tin oxide electrodes. Langmuir, 1998. 14(11): p. 8.

26. Muzyka, K., Current Trends in the Development of the Electrochemiluminescent Immunosensors. Biosensors and Bioelectronics, 2013.

27. Bian, J., et al., Double-potentiostatic electrodeposition of Ag nanoflowers on ITO glass for reproducible surface-enhanced (resonance) Raman scattering application. Electrochimica Acta, 2012. 67: p. 12-17.

28. Jingdong Zhang, A.M.K., Igor G. Medvedev, Qijin Chi, Tim Albrecht, Palle S. Jensen, and Jens Ulstrup, Single-Molecule Electron Transfer in Electrochemical Environments. chemical reviews, 2008. 109: p. 2737-2791.

29. SERGE G. LEMAY, S.K., KLAUS MATHWIG, AND PRADYUMNA S. SINGH, Single-Molecule Electrochemistry: Present Status and Outlook. Accounts of chemical research, 2012. 46(2): p. 9.

30. Kiguchi, M. and S. Kaneko, Single molecule bridging between metal electrodes. Physical chemistry chemical physics : PCCP, 2012. 
31. J. J. GARCIA-JAREGO, J.J.N., A. F. ROIG, H. ScHoLLt and F. VICENTE, Impedance analysis of Prussian Blue films deposited on ITO electrodes. electrochemica acta, 1995. 40(9): p. 7.

32. Wooster, S.E.C.a.T.T., A New Way of Using ac Voltammetry To Study Redox Kinetics in Electroactive Monolayers. Analytical chemistry, 1998. 70: p. 4257-263.

33. Feinberg, E.T.S.a.B.A., Redox Properties of Several Bacterial Ferredoxins Using Square Wave Voltammetry. the Journal of biological chemistry, 1990. 265(24): p. 6.

34. Parveen and R. Kant, Theory for staircase voltammetry and linear scan voltammetry on fractal electrodes: Emergence of anomalous Randles-Sevik behavior. Electrochimica Acta, 2013. 111: p. 223-233.

35. Dhillon, S. and R. Kant, Theory of Double Potential Step Chronoamperometry at Rough Electrodes: Reversible Redox Reaction and Ohmic Effects. Electrochimica Acta, 2014.

36. Kant, R. and M. Merajul Islam, Theory of single potential step absorbance transient at an optically transparent rough and finite fractal electrode: EC' mechanism. Journal of Electroanalytical Chemistry, 2013.

37. Jadreško, D. and M. Zelić, Cyclic multipulse voltammetric techniques. Part I: Kinetics of electrode processes. Journal of Electroanalytical Chemistry, 2013. 707: p. 20-30. 
38. Jadreško, D. and M. Zelić, Cyclic multipulse voltammetric techniques. Part II: EC mechanism. Journal of Electroanalytical Chemistry, 2014. 714-715: p. 30-37.

39. James L. Anderson, L.A.C., Jr., Johna Leddy, Dynamic Electrochemistry Methodology and Application. Analytical chemistry, 1998. 70(12): p. 72.

40. Chen, S. and Y. Liu, Electrochemistry at nanometer-sized electrodes. Physical chemistry chemical physics : PCCP, 2013. 16(2): p. 635-52.

41. Delphine Schaming, C.R., Ryan T. Tucker, Stéphanie Lau-Truong, Jean Aubard, Michael J. Brett, Véronique Balland, and Benoît Limoges, Spectroelectrochemical Characterization of Small Hemoproteins Adsorbed within Nanostructured Mesoporous ITO Electrodes. Langmuir, 2012. 28: p. 14065-14072.

42. Marken, K.J.M.a.F., Accumulation and Reactivity of the Redox Protein Cytochrome c in Mesoporous Films of TiO 2 Phytate. LANGMUIR 2003. 19(10): p. 5.

43. Astuti, Y., et al., Cyclic voltammetry and voltabsorptometry studies of redox proteins immobilised on nanocrystalline tin dioxide electrodes. Bioelectrochemistry, 2004. 63(1-2): p. 55-9.

44. Pandiaraj, M., et al., Designing label-free electrochemical immunosensors for cytochrome c using nanocomposites functionalized screen printed electrodes. Biosensors \& bioelectronics, 2014. 54: p. 115-21.

45. Jiang, X., L. Zhang, and S. Dong, Assemble of poly(aniline-co-oaminobenzenesulfonic acid) three-dimensional tubal net-works onto ITO electrode 
and its application for the direct electrochemistry and electrocatalytic behavior of cytochrome c. Electrochemistry Communications, 2006. 8(7): p. 1137-1141.

46. Jeronimo Agrisuelas, J.J.G.a.-J.o., * David Gimenez-Romero, and and F. Vicente, Innovative combination of three alternating current relaxation techniques electrical charge, mass, and color impedance spectroscopy part II prussian blue everitts salt process. Journal of Phyiscal Chemistry C, 2009. 113: p. 8438-8446.

47. Hill, C.M., D.A. Clayton, and S. Pan, Combined optical and electrochemical methods for studying electrochemistry at the single molecule and single particle level: recent progress and perspectives. Physical chemistry chemical physics : PCCP, 2013. 15(48): p. 20797-807.

48. Berkes, B.B., S. Vesztergom, and G. Inzelt, Combination of Nanogravimetry and Visible Spectroscopy: a Tool for the Better Understanding of Electrochemical Processes. Journal of Electroanalytical Chemistry, 2014.

49. Tautgirdas Ruzgas, L.W., Adolfas K. Gaigalas, and Vincent L. Vilker, Electron Transfer between Surface-Confined Cytochrome c and an N acetylcysteine modified gold electrode. langmuir, 1998. 14(25): p. 8.

50. Takamasa Sagma, S.I., Hisakuni Sato, and Katsumi Niki, Voltammetric application of electromodulated electroreflection absorption spectroscopy electroreflectance voltammetry as an in situ spectroelectrochemical technique. Langmuir, 1991. 7: p. 1005-1012. 
51. Heberle, K.A.a.J., Functional Vibrational Spectroscopy of a Cytochrome c Monolayer SEIDAS Probes the Interaction with Different Surface-Modified Electrodes. journal of the American Chemical Society, 2004. 126: p. 13.

52. Zhi Qiang Feng, T.S., and Katsumi NiKi, Application of potential modulated UV-Visible reflectance spectroscopy to electron transfer rate measurements for adsorbed species on electrode surfaces. Analytical Chemistry, 1995. 67(19): p. 7.

53. T. Amemiya, K.H., and A. Fujishima, Frequency-resolved faradaic processes in polypyrrole films observed by electromodulation techniques electrochemical impedance and color impedance spectroscopies. journal of physical chemistry, 1993. 97: p. 5.

54. Jain, A., et al., Visible spectroelectrochemical characterization of Geobacter sulfurreducens biofilms on optically transparent indium tin oxide electrode. Electrochimica Acta, 2011. 56(28): p. 10776-10785.

55. Ross, S.E., et al., Spectroelectrochemical sensing: planar waveguides. Electrochimica Acta, 2003. 48(20-22): p. 3313-3323.

56. Darren R. Dunphy, S.B.M., S. Scott Saavedra, and Neal R. Armstrong, The Electroactive Integrated Optical Waveguide Ultrasensitive Spectroelectrochemistry of Submonolayer Adsorbates Analytical chemistry, 1997. 69: p. 9.

57. Busalmen, J.P., et al., ATR-SEIRAs characterization of surface redox processes in G. sulfurreducens. Bioelectrochemistry, 2010. 78(1): p. 25-9. 
58. Beam, B.M., Waveguide sensor platforms: A) Development of the electroactive fiber-optic chip and B) Attenuated total reflectance spectroscopy of new molecular materials in Chemistry. 2008, University of Arizona.

59. Yining Shi, A.F.S., Carl J. Seliskar, and William R. Heineman, Spectroelectrochemical Sensing Based on Multimode Selectivity Simultaneously Achievable in a Single Device. 1. Demonstration of Concept with Ferricyanide. Analytical chemistry, 1997. 69: p. 3679-3686.

60. Zeynep Ozkan Araci, C.R.S., Neal R. Armstrong, and S. Scott Saavedra, Potential-Modulated Attenuated Total Reflectance Characterization of Charge Injection Processes in Monolayer-Tethered CdSe Nanocrystals. the Journal of Physical Chemistry Letters, 2010. 1: p. 6.

61. Matsuda, N., et al., Spectroelectrochemical studies on surface immobilized cytochrome c on ITO electrode by slab optical waveguide spectroscopy. Thin Solid Films, 2003. 438-439: p. 403-406.

62. Lei, C., D. Hu, and E.J. Ackerman, Single-molecule fluorescence spectroelectrochemistry of cresyl violet. Chem Commun (Camb), 2008(43): p. 54902.

63. Wang, X., et al., A new transfer function in electrochemistry: Dynamic coupling between Raman spectroscopy and electrochemical impedance spectroscopy. Electrochimica Acta, 2010. 55(21): p. 6299-6307.

64. Jiang, X., et al., Surface-enhanced resonance Raman spectroscopy and spectroscopy study of redox-induced conformational equilibrium of cytochrome c 
adsorbed on DNA-modified metal electrode. Biosensors \& bioelectronics, 2006. 22(1): p. 49-55.

65. Stockburger, P.H.a.M., Cytochrome c at Charged Interfaces. 1. Conformational and Redox Equilibria at the electrode electrolyte interface probed by surfaceenhanced resonance raman specroscopy. biochemistry, 1989. 28(12).

66. Królikowska, A., Surface-enhanced resonance Raman scattering (SERRS) as a tool for the studies of electron transfer proteins attached to biomimetic surfaces:, Case of cytochrome c. Electrochimica Acta, 2013.

67. Grützke, S., et al., Detection of DNA hybridization using electrochemical impedance spectroscopy and surface enhanced Raman scattering. Electrochemistry Communications, 2012. 19: p. 59-62.

68. Rahber Thariani, P.Y., Imaging of Surfaces by Concurrent Surface Plasmon Resonance and Surface Plasmon Resonance-Enhanced Fluorescence. PLoS ONE, 2010.5(3): p. 4.

69. Fujimaki, M., et al., The design of evanescent-field-coupled waveguide-mode sensors. Nanotechnology, 2008. 19(9): p. 095503.

70. Oh, Y., et al., Self-aligned colocalization of 3D plasmonic nanogap arrays for ultra-sensitive surface plasmon resonance detection. Biosensors \& bioelectronics, 2014. 51C: p. 401-407. 
71. Schneider, M., et al., Following fast adsorption processes with surface plasmon spectroscopy: reflectivity versus mismatch tracking. Sensors and Actuators B: Chemical, 2005. 104(2): p. 276-281.

72. Shao, Y., et al., Wavelength-multiplexing phase-sensitive surface plasmon imaging sensor. Optics Letters, 2013. 38(9): p. 1370.

73. Marek Piliarik, H.Š., Pavel Kvasnička, Nicolle Galler, Joachim R. Krenn, and Jiří Homola, High-resolution biosensor based on localized surface plasmons. optics express, 2012. 20(1): p. 672-680.

74. Jae Heon Ahn, T.Y.S., Won Mok Kim, Taek Sung Lee, Inho Kim, and and K.-S. Lee, Fiber-optic waveguide coupled surface plasmon resonance sensor. optics express, 2012. 20(19): p. 10.

75. Yan, H., et al., Research on Surface Plasmon Resonance Sensor Based on Wavelength Modulation by Using Theoretical Simulation Resonance Sensor Based on Wavelength Modulation by Using Theoretical Simulation. 2011: p. 83-86.

76. Chien, F.C., et al., Coupled waveguide-surface plasmon resonance biosensor with subwavelength grating. Biosensors \& bioelectronics, 2007. 22(11): p. 2737-42.

77. Sereda, A., et al., High performance multi-spectral interrogation for surface plasmon resonance imaging sensors. Biosensors \& bioelectronics, 2014. 54: p. 17580. 
78. Colin J. Alleyne, P.J.R.R., Sandrine Filion-Côté, and Andrew G. Kirk, Analysis of surface plasmon spectro-angular reflectance spectrum real time measurement, resolution limits, and applications to biosensing. optics Letters, 2011. 36(1): p. 3.

79. J. Homola, H.B.L.a.S.S.Y., Dual-channel surface plasmon resonance sensor with spectral discrimination of sensing channels using a dielectric overlayer. electronics letters, 1999. 35(13): p. 2.

80. H.P.Ho, S.Y.W., A.C.Cheung, Application of white light-emitting diode to surfaceplasmonresonance sensors. sensors and Actuators B: Chemical, 2001. 80: p. 6.

81. Huang Yan, G.L.-X., Su Wen-ling, Li Song-quan, and Ye Hong-an. Realization of Surface Plasmon Resonance Sensor Based on Wavelength Modulation by using 50 nm broadband light source. in IEEE. 2010.

82. Bouhelier, A. and G. Wiederrecht, Excitation of broadband surface plasmon polaritons: Plasmonic continuum spectroscopy. Physical Review B, 2005. 71(19).

83. HAN ZHANG, K.S.O., HIROMI TAKAHASHI, and S. SCOTT SAAVEDRA, Broadband Plasmon Waveguide Resonance Spectroscopy for Probing Biological Thin Films. Applied Spectroscopy, 2009. 63(9): p. 6.

84. Zhi-mei Qi, S.X., Mingdeng Wei, Hirofumi Matsuda, and Haoshen Zhou, Systematic characterization of spectral surface plasmon resonance sensors with absorbance measurement. Applied optics, 2007. 46(32): p. 7. 
85. A-P. Blanchard-Dionne, L.G., S. Patskovsky, R. Gordon, and M. Meunier, Intensity based surface plasmon resonance sensor using a nanohole rectangular array. opitcs express, 2011.19(16): p. 6.

86. Moirangthem, R.S., et al., Surface plasmon resonance ellipsometry based sensor for studying biomolecular interaction. Biosensors \& bioelectronics, 2010. 25(12): p. 2633-8.

87. Zheng Zheng, Y.W., Xin Zhao, and Jinsong Zhu, Spectral interferometric measurement of wavelength-dependent phase response for surface plasmon resonance sensors. Applied optics, 2009. 48(13): p. 5.

88. Zhang, H.Q., S. Boussaad, and N.J. Tao, High-performance differential surface plasmon resonance sensor using quadrant cell photodetector. Review of Scientific Instruments, 2003. 74(1): p. 150.

89. Homola, J., Present and future of surface plasmon resonance biosensors. Analytical and bioanalytical chemistry, 2003. 377(3): p. 528-39.

90. De Leon, I. and P. Berini, Theory of surface plasmon-polariton amplification in planar structures incorporating dipolar gain media. Physical Review B, 2008. 78(16).

91. Berini, I.D.L.a.P., Amplification of long-range surface plasmons by a dipolar gain medium. Nature Photonics, 2010. 4: p. 6.

92. Kasry, A. and W. Knoll, Long range surface plasmon fluorescence spectroscopy. Applied Physics Letters, 2006. 89(10): p. 101106. 
93. Huang, C.J., J. Dostalek, and W. Knoll, Optimization of layer structure supporting long range surface plasmons for surface plasmon-enhanced fluorescence spectroscopy biosensors. Journal of Vacuum Science \& Technology B: Microelectronics and Nanometer Structures, 2010. 28(1): p. 66.

94. Kwang-Hyon Kim, A.H., and Joachim Herrmann, Theory of plasmonic femtosecond pulse generation by mode-locking of long-range surface plasmon polariton lasers. optics express, 2012. 20(1): p. 12.

95. Malte C. Gather, K.M., Norbert Danz and Kristjan Leosson, Net optical gain in a plasmonic waveguide embedded in a fluorescent polymer. Nature Photonics, 2010.

96. Tollin, Z.S.a.G., Optical Anisotropy in Lipid Bilayer Membranes: Coupled Plasmon-Waveguide Resonance Measurements of Molecular Orientation, Polarizability, and Shape. Biophysical journal, 2001. 80: p. 11.

97. Zdzislaw Salamon, H.A.M., and Gordon Tollin, Coupled Plasmon-Waveguide Resonators: A New Spectroscopic Tool for Probing Proteolipid Film Structure and Properties. Biophysical journal, 1997. 73: p. 7.

98. Shigeru Toyama, N.D., Atsumu Shoji, Yoshihito Ikariyama, Design and fabrication of a waveguide-coupled prism device for surface plasmon resonance sensor. sensors and Actuators B: Chemical, 2000. 65: p. 3.

99. Chien, F.C. and S.J. Chen, A sensitivity comparison of optical biosensors based on four different surface plasmon resonance modes. Biosensors \& bioelectronics, 2004. 20(3): p. 633-42. 
100. Darvill, D., A. Centeno, and F. Xie, Plasmonic fluorescence enhancement by metal nanostructures: shaping the future of bionanotechnology. Physical chemistry chemical physics : PCCP, 2013. 15(38): p. 15709-26.

101. Garcia, H. and R. Sureshkumar, Self-consistent determination of plasmonic resonances in ternary nanocomposites. Physical Review B, 2007. 75(4).

102. Jain, P.K., et al., Review of Some Interesting Surface Plasmon Resonanceenhanced Properties of Noble Metal Nanoparticles and Their Applications to Biosystems. Plasmonics, 2007. 2(3): p. 107-118.

103. Gandra, N., et al., Plasmonic planet-satellite analogues: hierarchical selfassembly of gold nanostructures. Nano letters, 2012. 12(5): p. 2645-51.

104. Stobiecka, M., Novel plasmonic field-enhanced nanoassay for trace detection of proteins. Biosensors \& bioelectronics, 2013. 55C: p. 379-385.

105. Y. Zhang, T.Q.J., S.A. Zhang, D.H. Feng, and Z. Z. Xu, Dipole, quadrupole and octupole plasmon resonance modes in non-concentric nanocrescent nanodisk structure local field enhancement. optics express, 2012. 20(3): p. 8.

106. Yuan-Fong Chau, Y.-J.L.a.D.P.T., Enhanced surface plasmon resonance based on the silver nanoshells connected by the nanobars. opitcs express, 2012. 18(4): p. 9.

107. Krohne-Nielsen, P., et al., Surface-enhanced Raman microscopy of hemispherical shells stripped from templates of anodized aluminum. Journal of Raman Spectroscopy, 2012. 43(7): p. 834-341. 
108. Wong, C.L., et al., Colorimetric surface plasmon resonance imaging (SPRI) biosensor array based on polarization orientation. Biosensors \& bioelectronics, 2013. 47: p. 545-52.

109. A.A. Kruchinin, Y.G.V., Surface plasmon resonance monitoring by means of polarization state measurement in reflected light as the basis of a DNA-probe biosensor. sensors and Actuators B: Chemical, 1996. 30: p. 77-80.

110. Salamon, Z., H.A. Macleod, and G. Tollin, Coupled plasmon-waveguide resonators: A new spectroscopic tool for probing proteolipid film structure and properties. Biophysical Journal, 1997. 73(5): p. 2791-2797.

111. Zhang, H., et al., Broadband Plasmon Waveguide Resonance Spectroscopy for Probing Biological Thin Films. Applied Spectroscopy, 2009. 63(9): p. 1062-1067.

112. Hruby, V.J., et al., Use of plasmon waveguide resonance (PWR) spectroscopy for examining binding, signaling and lipid domain partitioning of membrane proteins. Life Sciences, 2010. 86(15-16): p. 569-574.

113. Aslan, M.M., et al., Low-Loss Optical Waveguides for the Near Ultra-Violet and Visible Spectral Regions with $\mathrm{Al}(2) \mathrm{O}(3)$ Thin Films from Atomic Layer Deposition. Thin Solid Films, 2010. 518(17): p. 6.

114. Fujishima, K.I.a.A., An Application Of Optical Wave-Guides To Electrochemistry - Construction Of Optical Wave-Guide Electrodes. the journal of physical chemistry, 1988. 92(25): p. 3. 
115. Hiroyuki Tomonaga, T.M., Indium tin oxide coatings via chemical solution deposition. Thin Solid Films, 2001. 392: p. 6.

116. Jeffrey W. Elam, D.A.B., Alex B. F. Martinson, Michael J. Pellin, and and J.T. Hupp, Atomic Layer Deposition of Indium Tin Oxide Thin Films Using Nonhalogenated presursors. journal of physical chemistry C, 2008. 112(6): p. 8.

117. T.C. Gorjanca, D.L., C. Py , D. Roth, Room temperature deposition of ITO using r.f. magnetron sputtering. Thin Solid Films, 2002. 413: p. 5.

118. Chung, C.-H., et al., Radio frequency magnetron sputter-deposited indium tin oxide for use as a cathode in transparent organic light-emitting diode. Thin Solid Films, 2005. 491(1-2): p. 294-297.

119. Keran Zhang, F.Z., U, C.H.A. Huan, A.T.S. Wee, Indium tin oxide films prepared by radio frequency magnetron sputtering method at a low processing temperature. Thin Solid Films, 2000. 376: p. 9.

120. Lee, S.K.C.a.J.I., Effect of film density on electrical properties of indium tin oxide films deposited by dc magnetron reactive sputtering. Journal of Vacuum Science \& Technology A: Vacuum, Surfaces, and Films, 2001. 19(5): p. 2043.

121. C. May, J.S., ITO coating by reactive magnetron sputtering-comparison of properties from DC and MF processing. Thin Solid Films, 1999. 351: p. 5.

122. S. Ishibashi, Y.H., Y. Ota, and K. Nakamura, Low resistivity indium-tin oxide transparent conductive films. II. Effect of sputtering voltage on electrical property of 
films. journal of Vacuum Science \& Technology A: Vacuum, Surfaces, and Films, 1989. 8(3): p. 4.

123. M. Bendera, W.S., C. Daube, H. Frankenberger, B. Ocker, J. Stollenwerk, Dependence of oxygen flow on optical and electrical properties of DC-magnetron sputtered ITO films. Thin Solid Films, 1998. 326: p. 6.

124. Cruz, L.R., et al., Influence of pressure and annealing on the microstructural and electro-optical properties of RF magnetron sputtered ITO thin films. Materials Research Bulletin, 2004. 39(7-8): p. 993-1003.

125. John C. C. Fan, F.J.B., and George H. Foley, Effect of 02 pressure during deposition on properties of rf-sputtered Sn-doped In203 films. Applied Physics Letters, 1977. 31(11): p. 3.

126. Herrero, C.G.a.J., Structure, optical, and electrical properties of indium tin oxide thin films prepared by sputtering at room temperature and annealed in air or nitrogen. Journal of Applied Physics, 2007. 101(7): p. 073514.

127. Paine, D.C., et al., A study of low temperature crystallization of amorphous thin film indium-tin-oxide. Journal of Applied Physics, 1999. 85(12): p. 8445.

128. Swanepoel, R., Determination of the thickness and optical constants of amorphous silicon. Journal of Physics E: Scientific Instruments, 1983. 16: p. 10.

129. Guo, E.-J., et al., Structure and characteristics of ultrathin indium tin oxide films. Applied Physics Letters, 2011. 98(1): p. 011905. 
130. Ow-Yang, C.W., et al., A time-resolved reflectivity study of the amorphous-tocrystalline transformation kinetics in dc-magnetron sputtered indium tin oxide. Journal of Applied Physics, 1998. 83(1): p. 145.

131. Liang, C.-H., et al., Influence of film thickness on the texture, morphology and electro-optical properties of indium tin oxide films. Thin Solid Films, 2010. 519(1): p. 345-350.

132. Pereira, M.B., J.S. Craven, and S.B. Mendes, Solid immersion lens at the aplanatic condition for enhancing the spectral bandwidth of a waveguide grating coupler. Optical Engineering, 2010. 49(12): p. 124601.

133. John Thomas Bradshaw, S.B.M., and S. Scott Saavedra, A Simplified Broadband Coupling Approach Applied to Chemically Robust Sol-Gel, Planar Integrated Optical Wveguides. Analytical chemistry, 2002. 74(8): p. 9.

134. Sergio B. Mendes, L.L., and James J. Burke, Broad-Band Attenuated Total Reflection Spectroscopy of a Hydrated Protein Film on a Single Mode Planar Waveguide. Langmuir 1996. 12: p. 3.

135. Saavedra, S.B.M.a.S.S., Comparative analysis of absorbance calculations for integrated optical waveguide configurations by use of the ray opticas model and the electromagnetic wave theory. Applied optics, 2000. 39(4): p. 10.

136. Darren Dunphy, S.M., S.Scott Saavedra and Neal R. Armstrong, spectroelectrochemistry of monolayer and submonolayer films using an electroactive integrated optical waveguide. 1999. 
137. Sergio B. Mendes, S.S.S., and Neal R. Armstrong, Broadband Spectroelectrochemical Interrogation of Molecular Thin Films by Single-Mode Electro-Active Integrated Optical Waveguides, in Optical Guided-wave Chemical and Biosensors. 2009.

138. Armstrong, N.R., et al., Interface modification of ITO thin films: organic photovoltaic cells. Thin Solid Films, 2003. 445(2): p. 342-352.

139. J. Pla , M.T., R. Rizzoli, M. Losurdo, E. Centurioni, C. Summonte, F. Rubinelli, Optimization of ITO layers for applications in a-Si c-Si heterojunction solar cells. Thin Solid Films, 2003. 425: p. 8.

140. Taylor, D.M. and C.A. Mills, Memory effect in the current-voltage characteristic of a low-band gap conjugated polymer. Journal of Applied Physics, 2001.90(1): p. 306.

141. Granqvist, C.G., Transparent conductive electrodes for electrochromic devices a review. Applied Physics A, 1993. 57: p. 6.

142. Yoon-Heung Taka, K.-B.K., Hyoung-Guen Parka, Kwang-Ho Leeb, Jong-Ram Lee, Criteria for ITO (indium-tin-oxide) thin film as the bottom electrode of an organic light emitting diode. Thin Solid Films, 2002. 411: p. 5.

143. Besbes, S., et al., Injection modifications by ITO functionalization with a selfassembled monolayer in OLEDs. Synthetic Metals, 2003. 138(1-2): p. 197-200. 
144. D Vaufrey, M.B.K., J Tardy, C Ghica, M G Blanchin, C Sandu and J A Roger, ITOon-top organic light-emitting devices a correlated study of opto-electronic and structural characteristics. Semiconductor Science and Technology, 2003. 18: p. 9.

145. Park, M., et al., Ultrafast laser ablation of indium tin oxide thin films for organic light-emitting diode application. Optics and Lasers in Engineering, 2006. 44(2): p. 138-146.

146. Tarwal, N.L. and P.S. Patil, Enhanced photoelectrochemical performance of $\mathrm{Ag}-\mathrm{ZnO}$ thin films synthesized by spray pyrolysis technique. Electrochimica Acta, 2011. 56(18): p. 6510-6516.

147. Zhengmao Yin, X.L., Yongzhong $\mathrm{Wu}$, Xiaopeng Hao, and Xiangang $\mathrm{Xu}$, Enhancement of light extraction in GaN-based light-emitting diodes using rough beveled ZnO nanocone arrays. Optics Express, 2011. 20(2): p. 9.

148. Jana, S.K., T. Majumder, and S. Banerjee, Enhanced photoelectrochemical property of gold nanoparticle sensitized Ti02 nanotube: A crucial investigation at electrode-electrolyte interface. Journal of Electroanalytical Chemistry, 2014. 727: p. 99-103.

149. Minami, T., Transparent conducting oxide semiconductors for transparent electrodes. Semiconductor Science and Technology, 2005. 20(4): p. S35-S44.

150. Cheng, H.-C., C.-F. Chen, and C.-Y. Tsay, Transparent ZnO thin film transistor fabricated by sol-gel and chemical bath deposition combination method. Applied Physics Letters, 2007. 90(1): p. 012113. 
151. Zudans, I., et al., Electrochemical and optical evaluation of noble metal- and carbon-ITO hybrid optically transparent electrodes. Journal of Electroanalytical Chemistry, 2004. 565(2): p. 311-320.

152. VANMAEKELBERGH, W.P.G.a.D., Impedance spectroscopy at semiconductor electrodes Review and recent developments. Electrochimica Acta, 1996. 41(7/8): p. 7.

153. C.G. Granqvist, A.H.k., Transparent and conducting ITO films new developments and applications. Thin Solid Films, 2002. 411: p. 5.

154. Byung Kee Lee, Y.H.S., and Jun Bo Yoon, Indium Tin Oxide (ITO) Transparent MEMS Switches, in IEEE. 2009.

155. B. Jonathan Luff, J.S.W., and Guido Perrone, Indium tin oxide overlayered waveguides for sensor applications. applied optics 1997.36(27): p. 7.

156. Vaishnav, V.S., P.D. Patel, and N.G. Patel, Indium Tin Oxide thin film gas sensors for detection of ethanol vapours. Thin Solid Films, 2005. 490(1): p. 94-100.

157. Betz, U., et al., Thin films engineering of indium tin oxide: Large area flat panel displays application. Surface and Coatings Technology, 2006. 200(20-21): p. 5751-5759.

158. Yu, J.W.L.a.J.S., Indium tin oxide subwavelength nanostructures with surface antireflection and superhydrophilicity for high-efficiency Si-based thin film solar cells. optics express, 2011. 20(53): p. 10. 
159. Ngamsinlapasathian, S., et al., Doubled layered ITO/SnO2 conducting glass for substrate of dye-sensitized solar cells. Solar Energy Materials and Solar Cells, 2006. 90(14): p. 2129-2140.

160. Jeong, J.-A. and H.-K. Kim, Low resistance and highly transparent ITO-Ag-ITO multilayer electrode using surface plasmon resonance of Ag layer for bulkheterojunction organic solar cells. Solar Energy Materials and Solar Cells, 2009. 93(10): p. 1801-1809.

161. Nüesch, F., et al., Importance of indium tin oxide surface acido basicity for charge injection into organic materials based light emitting diodes. Journal of Applied Physics, 2000. 87(11): p. 9.

162. Kim, J.S., et al., Indium-tin oxide treatments for single- and double-layer polymeric light-emitting diodes: The relation between the anode physical, chemical, and morphological properties and the device performance. Journal of Applied Physics, 1998. 84(12): p. 6859.

163. Arunandan Kumar, R.S., M. N. Kamalasanan, and Dalip Singh Mehta, Enhancement of light extraction efficiency of organic light emitting diodes using nanostructured indium tin oxide. opitcs express, 2012. 37(4): p. 3.

164. Psuja, P., D. Hreniak, and W. Strek, Synthesis and characterization of indiumtin oxide nanostructures. Journal of Physics: Conference Series, 2009. 146: p. 012033. 
165. S. Lauxa, N.K., A. ZoÈllerb, R. GoÈtzelmannb, H. Lauthc, H. Bernitzki, Roomtemperature deposition of indium tin oxide thin films with plasma ion-assisted evaporation. Thin Solid Films, 1998. 335: p. 5.

166. Hamberg, I., et al., Band-gap widening in heavily Sn-doped $\operatorname{In}_{-}\{2\} 0_{-}\{3\}$. Physical Review B, 1984. 30(6): p. 3240-3249.

167. Nüesch, F., et al., A photoelectron spectroscopy study on the indium tin oxide treatment by acids and bases. Applied Physics Letters, 1999. 74(6): p. 880.

168. Matveeva, E., Electrochemistry of the Indium-Tin Oxide Electrode in $1 \mathrm{M}$ NaOH Electrolyte. Journal of The Electrochemical Society, 2005. 152(9): p. H138.

169. Sharon E. Koh, K.D.M., David H. Holt, and Charles S. Dulcey, Phenylphosphonic Acid Functionalization of Indium Tin Oxide: surface chemistry and work function. Langmuir, 2006. 22: p. 7.

170. Dai Kato, G.X., Yuzuru Iwasaki, Yoshiki Hirata, Ryoji Kurita, and Osamu Niwa, Heavy Phosphate Adsorption on Amorphous ITO Film Electrodes Nano-Barrier Effect for Highly Selective Exclusion of Anionic Species. langmuir 2007. 23(16): p. 6.

171. Michael Brumbach, P.A.V., F. Saneeha Marrikar, Thomas Schulmeyer, and W.X. Adam Simmonds, Paul Lee, and Neal R. Armstrong, Surface Composition and Electrical and Electrochemical Properties of Freshly Deposited and Acid-Etched Indium Tin Oxide Electrodes. Langmuir, 2007. 23: p. 11. 
172. Bohn, A.L.S.a.P.W., Effect of the Interfacial Chemical Environment on In-Plane Electronic Conduction of Indium Tin Oxide Role of Surface Charge, Dipole Magnitude, and Carrier Injection. langmuir, 2004. 20: p. 9.

173. Cattarin, S., et al., Impedance Response of Resistive ITO Electrodes. Journal of The Electrochemical Society, 2011. 158(7): p. F135.

174. Harvey, S.P., et al., Surface versus bulk electronic/defect structures of transparent conducting oxides: I. Indium oxide and ITO. Journal of Physics D: Applied Physics, 2006. 39(18): p. 3959-3968.

175. Ramsden, S.S.a.J.J., Voltage-dependent coupling of light into ITO-covered waveguides. Journal of Physics D: Applied Physics, 2002. 35: p. 4.

176. Michelle A. Brusatori, P.R.V.T., Biosensing under an applied voltage using optical waveguide lightmode spectroscopy. Biosensors and Bioelectronics, 2003. 18(10): p. 1269-1277.

177. J. He, M.L., X. Zhou, J.R. Cao, K.L. Wang, L.S. Liao, Z.B. Deng, X.M. Ding, X.Y. Hou, S.T. Lee, Damage study of ITO under high electric field. The solid films, 2000. 363: p. 4.

178. H. Michel, J.B., A. Harrenga, and A. Kannt, CYTOCHROME C OXIDASE: structure and spectroscopy. annual review of biophysics and biomolecular structure, 1998. 27: p. 29.

179. LEMBERG, M.R., Cytochrome Oxidase. physiological reviews, 1969. 49(1). 
180. Alan S. Haas, D.L.P., Konda S. Reddy, Gerald T. Babcock, Christopher C. Moser, J. Kent Blasie, and P. Leslie Dutton, Cytochrome c and Cytochrome c Oxidase Monolayer Assemblies and Catalysis. JOURNAL OF PHYsical chemistry B, 2001. 105: p. 12.

181. Francis A. Haskins, A.T., Herschell K. Mitchell and Mary B. Mitchell, Cytochromes and the Succinic Acid Oxidase System of Poky Strains of Neurospora. Journal of Biological Chemistry, 1953. 200(2): p. 819-826.

182. Wang, L., The Denaturation of Cytochrome $\mathrm{c}$ and Cytochrome $\mathrm{c}$ as Peroxidase. 2006, UNIVERSITY OF PITTSBURGH.

183. Melin, F., et al., Direct electrochemistry of cytochrome bo3 oxidase at a series of gold nanoparticles-modified electrodes. Electrochemistry Communications, 2013. 26: p. 105-108.

184. Tautgirdas Ruzgas, A.G., Lo Gorton, Diffusionless electron transfer of microperoxidase-11 on gold electrodes. journal of Electroanalytical Chemistry, 1999. 469: p. 123-131.

185. Hildebrandt, D.H.M.a.P., Redox and redox-coupled processes of heme proteins and enzymes at electrochemical interfaces. Physical Chemistry Chemical Physics, 2005. 7: p. 3773-3748.

186. Huttemann, M., et al., The multiple functions of cytochrome $\mathrm{c}$ and their regulation in life and death decisions of the mammalian cell: From respiration to apoptosis. Mitochondrion, 2011. 11(3): p. 369-81. 
187. Ow, Y.P., et al., Cytochrome c: functions beyond respiration. Nature reviews. Molecular cell biology, 2008. 9(7): p. 532-42.

188. BALL, F.L.R.A.E.G., OXIDATION-REDUCTION POTENTIALS OF THE CYTOCHROME c SYSTEM. 1949.

189. Wen Jin, U.W., Eva Kargel, Wolf-Hagen Schunck, Frieder W. Scheller, Electrochemical investigations of the intermolecular electron transfer between cytochrome c and NADPH-cytochrome P450-reductase. journal of Electroanalytical Chemistry, 1997. 433: p. 135-139.

190. C. A. Yu, L.Y.a.T.E.K., Kinetics of Electron Transfer between Cardiac Cytochrome c1 and c. journal of biological chemistry, 1973. 248: p. 528-533.

191. Mohsin, M.A., et al., Electrochemical Impedance Spectroscopy for Assessing the Recognition of Cytochrome c by Immobilized Calixarenes. Electroanalysis, 2011. 23(5): p. 1229-1235.

192. HILDEBRANDT, D.H.M.A.P., Electron-transfer processes of cytochrome $\mathrm{c}$ at interfaces. New insights by surface-enhanced resonance Raman spectroscopy. Acc. Chem. Res, 2004. 37(8).

193. Margoliash, W.H.K.a.E., The asymmetric distribution of charges on the surface of horse cytochrome c. Functional implications. The Journal of biological chemistry, 1982. 257(8): p. 12.

194. Craig W. Herbold, J.H.M., Steven C. Goheen, Cytochrome c unfolding on an anionic surface. Chromatography A, 1999. 863: p. 10. 
195. Bowden, M.C.a.E.F., UV-Visible Spectroscopy of Adsorbed Cytochrome c on Tin oxide electrode. Analytical Chemistry, 1992. 64(13): p. 7.

196. Shin-ichiro Imabayashi, T.M., and Takashi Kakiuchi, Effect of the Electrostatic Interaction on the Redox Reaction of Positively Charged Cytochrome c Adsorbed on the Negatively Charged Surfaces of Acid-Terminated Alkanethiol Monolayers on a $\mathrm{Au}(111)$ Electrode. langmuir, 2005. 21: p. 5.

197. FROHWIRT, E.M.A.N., Spectrum of Horse-Heart Cytochrome c. biochemical journal, 1959. 71(3): p. 2.

198. T. Sagara, K.N., A. Sone, C. Hinnen, and K. Niki, Redox Reaction-Mechanism Of Cytochrome-C At Modified Gold Electrodes. Langmuir, 1990. 6: p. 9.

199. Allen J. Bard, L.R.F., Electrochemical methods fundamentals and applications. 2001.

200. Bowden, R.A.C.a.E.F., Voltammetric Peak Broadening for Cytochrome c Alkanethiolate Monolayer Structures Dispersion of Formal Potentials. Langmuir, 1997. 13: p. 7.

201. Hildebrandt, D.H.M.a.P., Electrostatic-Field Dependent Activation Energies Modulate Electron Transfer of Cytochrome c. journal of physical chemistry B, 2002. 106: p. 12814-12819.

202. Lee, J.C., H.B. Gray, and J.R. Winkler, Cytochrome c' folding triggered by electron transfer: fast and slow formation of four-helix bundles. Proceedings of the 
National Academy of Sciences of the United States of America, 2001. 98(14): p. $7760-4$

203. Casey M. Kraning, T.L.B., Kayla S. Bloome, Gregory C. Campanello,, et al., Determination of Surface Coverage and Orientation of Reduced Cytochrome c on a Silica Surface with Polarized ATR Spectroscopy. journal of physical chemistry C, 2007.111(35): p. 6.

204. DiCarlo, C.M., et al., Bioelectrocatalysis in ionic liquids. Examining specific cation and anion effects on electrode-immobilized cytochrome c. Bioelectrochemistry, 2006. 68(2): p. 134-43.

205. A. Szucs, M.N., Stable and reversible electrochemistry of cytochrome c on bare electrodes Part 1. Effect of ionic strength. journal of Electroanalytical Chemistry, 1995. 383: p. 10.

206. Shabir H. Qureshi, B.M., Sushma Yadav, and Faizan Ahmad, Conformational and Thermodynamic Characterization of the Molten Globule State Occurring during Unfolding of Cytochromes-c by Weak Salt Denaturants. biochemistry, 2003. 42: p. 12. 207. Saavedra, Y.-Z.D.a.S.S., Molecular Orientation Distributions in Protein Films. V. cytochrome c adsorbed on a sulonate-terminated, self-aseembled monolayer. Langmuir, 2004. 19: p. 6.

208. Anne F. Runge, N.C.R., S. Scott Saavedra, and Sergio B. Mendes, Determination of Anisotropic Optical Constants and Surface Coverage of Molecular Films Using Polarized Visible ATR Spectroscopy. Application to Adsorbed Cytochrome c Films. journal of physical chemistry B, 2005. 109: p. 8. 
209. Anne F. Runge, S.B.M., and S. Scott Saavedra, Order parameters and orientation distributions of solution adsorbed and microcontact printed cytochrome c protein films on glass and ITO. journal of physical chemistry B, 2006. 110: p. 8.

210. Caesar, C.E., et al., Assigning membrane binding geometry of cytochrome C by polarized light spectroscopy. Biophysical journal, 2009. 96(8): p. 3399-411.

211. J. G. E. M. Fraaije, J.M.K., M. van der Graaf, and J. C. Dijt, Orientation of adsorbed cytochrome $\mathrm{c}$ as a function of the electrical potential of the interface studied by total internal reflection fluorescence. biophysics, 1990. 57: p. 11.

212. Y.-Y. Cheng, S.H.L., H.-C. Chang, andM.-C. Su, Probing adsorption, orientation and conformational changes of cytochrome $\mathrm{c}$ on fused silica surfaces with the soret band. Journal of Physical Chemistry A, 2003. 107: p. 8.

213. Zeynep Ozkan Araci, A.F.R., Walter J. Doherty III, and S. Scott Saavedra, Correlating molecular orientation distributions and electrochemical kinetics in subpopulation of an immoblized protein film. journal of the American Chemical Society, 2007. 130: p. 2.

214. Bowden, J.X.a.E.F., Determination of the Orientation of Adsorbed Cytochrome c on Carboxyalkanethiol Self-Assembled Monolayers by In Situ Differential Modification. journal of the American Chemical Society, 2006. 128: p. 10.

215. Takamasa Sagara, Y.K., and Keizo Hiraishi, Estimation of the Orientation of Heme in Cytochrome c Immobilized on a Carboxylate-Terminated Alkanethiol Monolayer on a Au Electrode by the Use of Electroreflectance Spectroscopy with Polarized Light Incidence. journal of physical chemistry B, 2006. 110: p. 9. 
216. Lakowicz, J.R., Radiative decay engineering 5: metal-enhanced fluorescence and plasmon emission. Analytical biochemistry, 2005. 337(2): p. 171-94.

217. Gryczynski, I., et al., Radiative decay engineering 4. Experimental studies of surface plasmon-coupled directional emission. Analytical biochemistry, 2004. 324(2): p. 170-182.

218. Lakowicz, J.R., Radiative decay engineering 3. Surface plasmon-coupled directional emission. Analytical biochemistry, 2004. 324(2): p. 153-169.

219. Stolar, M. and T. Baumgartner, Organic n-type materials for charge transport and charge storage applications. Physical chemistry chemical physics : PCCP, 2013. 15(23): p. 9007-24.

220. Chein-Yie Chung, T.-C.W., A. Gopalan, Identification of electrochromic sites in poly(diphenylamine) using a novel absorbance-potential-wavelength profile. Electrochimica Acta, 2001. 47: p. 9.

221. J. Agrisuelas, C.G., J. J. Garcı́a-Jareno, D. Gimenez-Romero, H. Perrot, and F. Vicente, Spectroelectrochemical Identification of the Active Sites for Protons and Anions Insertions into Poly-(Azure A) Thin Polymer Films. journal of physical chemistry c, 2007.111: p. 14230-14237.

222. Tyan, Y.-S., Organic light-emitting-diode lighting overview. Journal of Photonics for Energy, 2011. 1(1): p. 011009.

223. M. Fahland, P.K., C. Charton, Low resisitivity transparent electrodes for displays on polymer substrates. Thin Solid Films, 2001. 392: p. 4. 
224. Hiroshi Imahori, M.K., Kohei Hosomizu, Tomoo Sato, Tae Kyu Ahn, Seong Keun Kim, Dongho Kim, YoshinobuNi shimura, Iwao Yamazaki, Yasuyuki Araki, Osamulto, and Shunichi Fukuzumi, Vectorial Electron Relay at ITO Electrodes Modified with Self-Assembled Monolayers of Ferrocene-Porphyrin-Fullerene Triads and Porphyrin-Fullerene Dyads for Molecular Photovoltaic Devices. Europen Journal of chemistry, 2004. 10: p. 12.

225. Lifeng Li, M.X., George I. Stegeman, and Colin T. Seaton. Fabrication of photoresist masks for submicrometer surface relief gratings. in SPIE. 1987. 


\section{APPENDIX}

Appendix 1: Mathematica program for the guided waveguide mode, effective refractive index, sensitivity and electric field distribution across the layers for a multi-layer structure with TE polarization incident light

Clear $[\lambda]$;

$\lambda=550$;

$n_{c}=1.33211$

$n_{1}=1.9 ; t_{1}=13$;

$n_{2}=1.51 ; t_{2}=16$;

$\mathrm{a}=1.64576 ; \mathrm{b}=42.89898 ; \mathrm{c}=308958233.142$;

$n_{2}=\mathrm{a}+\frac{\mathrm{b}}{\lambda^{2}}+c / \lambda^{4} ; t_{3}=400 ;$

$n_{s}=1.51$;

$\alpha_{c}=\sqrt{n_{c}^{2}-\beta^{2}} ;$

$\alpha_{1}=\sqrt{n_{1}^{2}-\beta^{2}}$;

$\alpha_{2}=\sqrt{n_{2}^{2}-\beta^{2}}$;

$\alpha_{3}=\sqrt{n_{3}^{2}-\beta^{2}}$;

$\alpha_{s}=\sqrt{n_{s}^{2}-\beta^{2}}$;

$\delta_{1}=\frac{2 \pi}{\lambda} \alpha_{1} t_{1}$;

$\delta_{2}=\frac{2 \pi}{\lambda} \alpha_{2} t_{2} ;$

$\delta_{3}=\frac{2 \pi}{\lambda} \alpha_{3} t_{3} ;$

$p_{c}=1 ; p_{c}=1 ; p_{c}=1 ; p_{c}=1 ; p_{c}=1 ;$

$q_{c}=-\alpha_{c} ; q_{1}=-\alpha_{1} ; q_{2}=-\alpha_{2} ; q_{3}=-\alpha_{3} ; q_{s}=-\alpha_{s} ;$ 


$$
\begin{aligned}
& M_{1}=\left(\begin{array}{cc}
\operatorname{Cos}\left[\delta_{1}\right] & \frac{-i p_{1}}{q_{1}} \operatorname{Sin}\left[\delta_{1}\right] \\
\frac{-i q_{1}}{p_{1}} \operatorname{Sin}\left[\delta_{1}\right] & \operatorname{Cos}\left[\delta_{1}\right]
\end{array}\right) ; \\
& M_{2}=\left(\begin{array}{cc}
\operatorname{Cos}\left[\delta_{2}\right] & \frac{-i p_{2}}{q_{2}} \operatorname{Sin}\left[\delta_{2}\right] \\
\frac{-i q_{2}}{p_{2}} \operatorname{Sin}\left[\delta_{2}\right] & \operatorname{Cos}\left[\delta_{2}\right]
\end{array}\right) ; \\
& M_{3}=\left(\begin{array}{cc}
\operatorname{Cos}\left[\delta_{3}\right] & \frac{-i p_{3}}{q_{3}} \operatorname{Sin}\left[\delta_{3}\right] \\
\frac{-i q_{3}}{p_{3}} \operatorname{Sin}\left[\delta_{3}\right] & \operatorname{Cos}\left[\delta_{3}\right]
\end{array}\right) ; \\
& D_{c}=\left(\begin{array}{cc}
p_{c} & p_{c} \\
q_{c} & -q_{c}
\end{array}\right) ; \\
& D_{s}=\left(\begin{array}{cc}
p_{s} & p_{s} \\
q_{s} & -q_{s}
\end{array}\right) ; \\
& M_{t}=\operatorname{Inverse}\left[D_{c}\right] \cdot M_{1} \cdot M_{2} \cdot M_{3} \cdot D_{s} ; \\
& m_{11}=\operatorname{Abs}\left[M_{t}[[1,1]]\right] ;
\end{aligned}
$$

$\operatorname{Plot}\left[m_{11},\left\{\beta, n_{s}, n_{3}\right\}\right]$;

FindRoot $\left[m_{11}==0,\left\{\beta, n_{s}+0.001, \operatorname{Max}\left[n_{1}, n_{2}, n_{3}\right]-0.001\right\}\right]$;

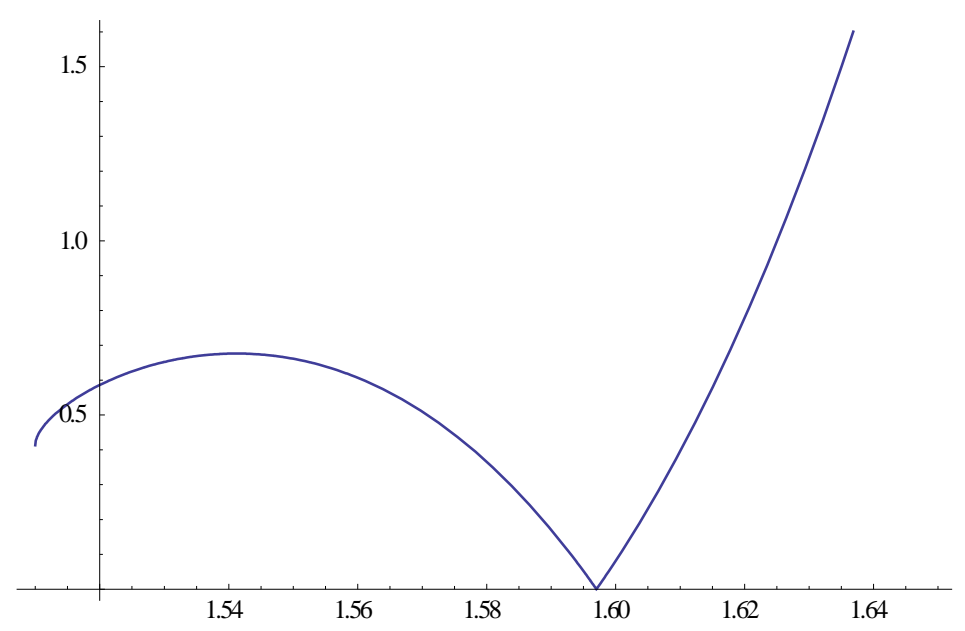

$\{\beta \rightarrow 1.59702\}$

Clear [z]

$\beta=1.5978$;

$\lambda=550$;

$n_{c}=1.33211$; 


$$
\begin{aligned}
& n_{1}=1.9 ; t_{1}=13 ; \\
& n_{2}=1.46 ; t_{2}=15 \text {; } \\
& \mathrm{a}=1.64576 ; \mathrm{b}=42.89898 ; \mathrm{c}=308958233.142 \text {; } \\
& n_{3}=\mathrm{a}+\frac{\mathrm{b}}{\lambda^{2}}+c / \lambda^{4} \text {; } \\
& t_{3}=15 \text {; } \\
& n_{s}=1.51 \\
& \eta_{c}=\sqrt{n_{c}^{2}-\beta^{2}} ; \\
& \eta_{1}=\sqrt{n_{1}^{2}-\beta^{2}} \text {; } \\
& \eta_{2}=\sqrt{n_{2}^{2}-\beta^{2}} \text {; } \\
& \eta_{3}=\sqrt{n_{3}^{2}-\beta^{2}} \text {; } \\
& \eta_{s}=\sqrt{n_{s}^{2}-\beta^{2}} \text {; } \\
& \delta_{1}=\frac{2 \pi}{\lambda} \alpha_{1} t_{1} \text {; } \\
& \delta_{2}=\frac{2 \pi}{\lambda} \alpha_{2} t_{2} \text {; } \\
& \delta_{3}=\frac{2 \pi}{\lambda} \alpha_{3} t_{3} ; \\
& M_{1}=\left(\begin{array}{cc}
\operatorname{Cos}\left[\delta_{1}\right] & \frac{i}{\eta_{1}} \operatorname{Sin}\left[\delta_{1}\right] \\
i \eta_{1} \operatorname{Sin}\left[\delta_{1}\right] & \operatorname{Cos}\left[\delta_{1}\right]
\end{array}\right) ; \\
& M_{2}=\left(\begin{array}{cc}
\operatorname{Cos}\left[\delta_{2}\right] & \frac{i}{\eta_{2}} \operatorname{Sin}\left[\delta_{2}\right] \\
i \eta_{2} \operatorname{Sin}\left[\delta_{2}\right] & \operatorname{Cos}\left[\delta_{2}\right]
\end{array}\right) ; \\
& M_{3}=\left(\begin{array}{cc}
\operatorname{Cos}\left[\delta_{3}\right] & \frac{i}{\eta_{2}} \operatorname{Sin}\left[\delta_{3}\right] \\
i \eta_{2} \operatorname{Sin}\left[\delta_{3}\right] & \operatorname{Cos}\left[\delta_{3}\right]
\end{array}\right) \text {; } \\
& Y_{s}=\left(\begin{array}{c}
1 \\
-\eta_{s}
\end{array}\right) \operatorname{Exp}\left[-i \frac{2 \pi}{\lambda} a_{s} z\right] \text {; } \\
& Y_{3}=\left(\begin{array}{c}
1 \\
-\eta_{s}
\end{array}\right) M_{3} \text {; } \\
& Y_{2}=\left(\begin{array}{c}
1 \\
-\eta_{s}
\end{array}\right)\left(\begin{array}{cc}
\operatorname{Cos}\left[\frac{2 \pi}{\lambda} a_{3} t_{3}\right] & \frac{i}{\eta_{3}} \operatorname{Sin}\left[\frac{2 \pi}{\lambda} a_{3} t_{3}\right] \\
i \eta_{3} \operatorname{Sin}\left[\frac{2 \pi}{\lambda} a_{3} t_{3}\right] & \operatorname{Cos}\left[\frac{2 \pi}{\lambda} a_{3} t_{3}\right]
\end{array}\right) M_{2} \\
& Y_{1}=M_{1}\left(\begin{array}{c}
1 \\
-\eta_{5}
\end{array}\right)\left(\begin{array}{cc}
\operatorname{Cos}\left[\frac{2 \pi}{\lambda} a_{2} t_{2}\right] & \frac{i}{\eta_{2}} \operatorname{Sin}\left[\frac{2 \pi}{\lambda} a_{2} t_{2}\right] \\
i \eta_{2} \operatorname{Sin}\left[\frac{2 \pi}{\lambda} a_{2} t_{2}\right] & \operatorname{Cos}\left[\frac{2 \pi}{\lambda} a_{2} t_{2}\right]
\end{array}\right)\left(\begin{array}{cc}
\operatorname{Cos}\left[\frac{2 \pi}{\lambda} a_{3} t_{3}\right] & \frac{i}{\eta_{3}} \operatorname{Sin}\left[\frac{2 \pi}{\lambda} a_{3} t_{3}\right] \\
i \eta_{3} \operatorname{Sin}\left[\frac{2 \pi}{\lambda} a_{3} t_{3}\right] & \operatorname{Cos}\left[\frac{2 \pi}{\lambda} a_{3} t_{3}\right]
\end{array}\right)
\end{aligned}
$$




$$
\begin{aligned}
& Y_{c}=\operatorname{Exp}\left[i \frac{2 \pi}{\lambda} a_{s} z\right]\left(\begin{array}{c}
1 \\
-\eta_{s}
\end{array}\right)\left(\begin{array}{cc}
\operatorname{Cos}\left[\frac{2 \pi}{\lambda} a_{1} t_{1}\right] & \frac{i}{\eta_{2}} \operatorname{Sin}\left[\frac{2 \pi}{\lambda} a_{1} t_{1}\right] \\
i \eta_{1} \operatorname{Sin}\left[\frac{2 \pi}{\lambda} a_{1} t_{1}\right] & \operatorname{Cos}\left[\frac{2 \pi}{\lambda} a_{1} t_{1}\right]
\end{array}\right) \\
& \left(\begin{array}{cc}
\operatorname{Cos}\left[\frac{2 \pi}{\lambda} a_{2} t_{2}\right] & \frac{i}{\eta_{2}} \operatorname{Sin}\left[\frac{2 \pi}{\lambda} a_{2} t_{2}\right] \\
i \eta_{2} \operatorname{Sin}\left[\frac{2 \pi}{\lambda} a_{2} t_{2}\right] & \operatorname{Cos}\left[\frac{2 \pi}{\lambda} a_{2} t_{2}\right]
\end{array}\right)\left(\begin{array}{cc}
\operatorname{Cos}\left[\frac{2 \pi}{\lambda} a_{3} t_{3}\right] & \frac{i}{\eta_{3}} \operatorname{Sin}\left[\frac{2 \pi}{\lambda} a_{3} t_{3}\right] \\
i \eta_{3} \operatorname{Sin}\left[\frac{2 \pi}{\lambda} a_{3} t_{3}\right] & \operatorname{Cos}\left[\frac{2 \pi}{\lambda} a_{3} t_{3}\right]
\end{array}\right)
\end{aligned}
$$

depth $=500$;

height $=2 \times 10^{7} / 500$;

normalization $=$ NIntegrate $\left[\left(\operatorname{Re}\left[Y_{S}[[1]]\right]\right)^{\wedge} 2,\{z,-\right.$ depth, 0$\left.\}\right]$

+ NIntegrate $\left[\left(\operatorname{Re}\left[Y_{3}[[1]]\right]\right)^{\wedge} 2,\left\{z, 0, t_{3}\right\}\right]$

+NIntegrate $\left[\left(\operatorname{Re}\left[Y_{2}[[1]]\right]\right)^{\wedge} 2,\left\{z, 0, t_{2}\right\}\right]$

+ NIntegrate $\left[\left(\operatorname{Re}\left[Y_{1}[[1]]\right]\right)^{\wedge} 2,\left\{z, 0, t_{1}\right\}\right]$

+NIntegrate $\left[\left(\operatorname{Re}\left[Y_{c}[[1]]\right]\right)^{\wedge} 2,\{z, 0, \operatorname{depth}\}\right]$;

$Y_{0}$

$$
\begin{aligned}
& =\left(\begin{array}{c}
1 \\
-\eta_{s}
\end{array}\right)\left(\begin{array}{cr}
\operatorname{Cos}\left[\frac{2 \pi}{\lambda} a_{1} t_{1}\right] & \frac{i}{\eta_{2}} \operatorname{Sin}\left[\frac{2 \pi}{\lambda} a_{1} t_{1}\right] \\
i \eta_{1} \operatorname{Sin}\left[\frac{2 \pi}{\lambda} a_{1} t_{1}\right] & \operatorname{Cos}\left[\frac{2 \pi}{\lambda} a_{1} t_{1}\right]
\end{array}\right)\left(\begin{array}{cc}
\operatorname{Cos}\left[\frac{2 \pi}{\lambda} a_{2} t_{2}\right] & \frac{i}{\eta_{2}} \operatorname{Sin}\left[\frac{2 \pi}{\lambda} a_{2} t_{2}\right] \\
i \eta_{2} \operatorname{Sin}\left[\frac{2 \pi}{\lambda} a_{2} t_{2}\right] & \operatorname{Cos}\left[\frac{2 \pi}{\lambda} a_{2} t_{2}\right]
\end{array}\right) \\
& \left(\begin{array}{cc}
\operatorname{Cos}\left[\frac{2 \pi}{\lambda} a_{3} t_{3}\right] & \frac{i}{\eta_{3}} \operatorname{Sin}\left[\frac{2 \pi}{\lambda} a_{3} t_{3}\right] \\
i \eta_{3} \operatorname{Sin}\left[\frac{2 \pi}{\lambda} a_{3} t_{3}\right] & \operatorname{Cos}\left[\frac{2 \pi}{\lambda} a_{3} t_{3}\right]
\end{array}\right)
\end{aligned}
$$

$\mathrm{g}_{s}=$ ParametricPlot $\left[\left\{z,\left(1 \times 10^{7} /\right.\right.\right.$ normalization $\left.)\left(\operatorname{Re}\left[Y_{s}[[1]]\right]\right)^{\wedge} 2\right\},\{z,-$

depth, 0$\}$,PlotRange $\rightarrow\left\{\left\{\right.\right.$ depth,depth $\left.+t_{1}+t_{2}+t_{3}\right\},\{0$, height $\left.\}\right\}$,AspectRatio $\rightarrow$ Full,PlotLa bel $\rightarrow$ field intensity distribution];

$\mathrm{g}_{3}=$ ParametricPlot $\left[\left\{z,\left(1 \times 10^{7} /\right.\right.\right.$ normalization $)\left(\operatorname{Re}\left[Y_{3}\right.\right.$

$\left.[[1]]])^{\wedge} 2\right\},\left\{z, 0, t_{3}\right\}$, PlotRange $\rightarrow\{\{-$

depth,depth $\left.+t_{1}+t_{2}+t_{3}\right\},\{0$, height $\left.\}\right\}$,AspectRatio $\rightarrow$ Full,PlotLabel $\rightarrow$ field intensity distribution];

$\mathrm{g}_{2}=$ ParametricPlot $\left[\left\{z+\mathrm{t}_{3},\left(1 \times 10^{7} /\right.\right.\right.$ normalization $)\left(\operatorname{Re}\left[Y_{2}\right.\right.$

$\left.[[1]]])^{\wedge} 2\right\},\left\{z, 0, t_{2}\right\}$, PlotRange $\rightarrow\{\{-$

depth,depth $\left.+t_{1}+t_{2}+t_{3}\right\},\{0$, height $\left.\}\right\}$,AspectRatio $\rightarrow$ Full,PlotLabel $\rightarrow$ field intensity distribution];

$\mathrm{g}_{1}=$ ParametricPlot $\left[\left\{z+\mathrm{t}_{2}+\mathrm{t}_{3},\left(1 \times 10^{7} /\right.\right.\right.$ normalization $)\left(\operatorname{Re}\left[Y_{1}\right.\right.$

$\left.[[1]]])^{\wedge} 2\right\},\left\{z, 0, t_{1}\right\}$, PlotRange $\rightarrow\{\{-$

depth,depth $\left.+t_{1}+t_{2}+t_{3}\right\},\{0$, height $\left.\}\right\}$,AspectRatio $\rightarrow$ Full,PlotLabel $\rightarrow$ field intensity distribution];

$\mathrm{gc}=$ ParametricPlot $\left[\left\{z+\mathrm{t}_{1}+\mathrm{t}_{2}+\mathrm{t}_{3},\left(1 \times 10^{7} /\right.\right.\right.$ normalization $)\left(\operatorname{Re}\left[Y_{c}\right.\right.$ $\left.[[1]]])^{\wedge} 2\right\},\{z, 0$, depth $\}$, PlotRange $\rightarrow\{\{-$ 
depth,depth $\left.+t_{1}+t_{2}+t_{3}\right\},\{0$,height $\left.\}\right\}$, AspectRatio $\rightarrow$ Full,PlotLabel $\rightarrow$ field intensity distribution];

$\mathrm{g}_{0}=$ ParametricPlot $\left[\left\{z+t_{1}+t_{2}+t_{3},\left(1 \times 10^{7} /\right.\right.\right.$ normalization $\left.)\left(\operatorname{Re}\left[Y_{0}[[1]]\right]\right)^{\wedge} 2\right\},\{z,-$ $10,10\}$,PlotRange $\rightarrow\left\{\left\{\right.\right.$-depth,depth $\left.+\mathrm{t}_{1}+\mathrm{t}_{2}+\mathrm{t}_{3}\right\},\{0$, height $\left.\}\right\}$, AspectRatio $\rightarrow$ Full, PlotStyle $\rightarrow$ Red,PlotLabel $\rightarrow$ field intensity distribution];

Show $\left[\mathrm{g}_{s}, \mathrm{~g}_{3}, \mathrm{~g}_{2}, \mathrm{~g}_{1}, \mathrm{~g}_{c}, \mathrm{~g}_{0}\right]$

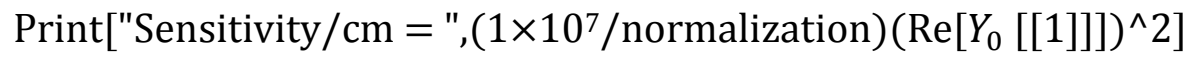

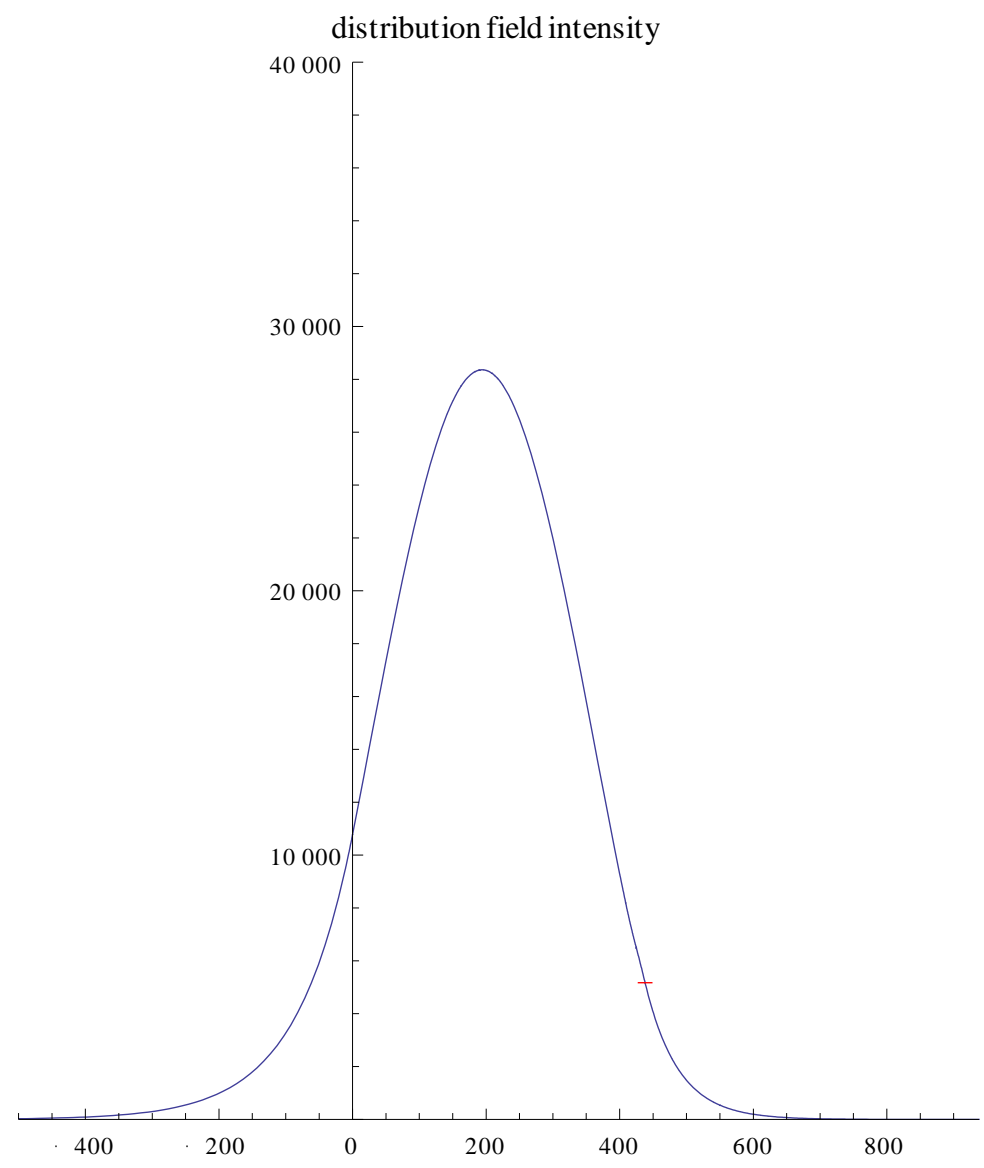

Sensitivity $/ \mathrm{cm}=\{5176.2\}$ 
Appendix 2: Surface relief grating fabrication

Micro glass slides (VWR) were used as the substrates. To avoid any propagation loss from the surface scratches, the glass slides were examined first. Qualified glass slides were put in the heated $\left(60^{\circ} \mathrm{C}\right)$ micro-detergent solution for 10 minutes of sonication and then wiped with cotton tips. The same step was repeated and followed by a DI water rinse. The slides were then put into heated $\left(90^{\circ} \mathrm{C}\right)$ piranha solution for 10 minutes and then rinsed with DI water. The slides were put into heated $\left(60^{\circ} \mathrm{C}\right)$ methanol solution for 10 minutes of sonication and blown dry with $\mathrm{N}_{2}$ gas. After this cleaning procedure, both organic and inorganic contaminations were cleaned. Cleaned slides were put into an oven for 1 hour to minimize surface moisture for photoresist (1805) spinning process. Another heating process in oven was applied to promote the photoresist adhesion to the glass surface after the spinning. A layer of black paint was applied on the back side of the glass slide to minimize the backside reflection during the UV exposure. The holography set-up to expose photoresist for the grating pattern was described in Appendix Figure 1(a). The exposure source was a He-Cd laser with $442 \mathrm{~nm}$. An objective lens was used to focus the laser beam into a $10 \mu \mathrm{m}$ pin-hole to cut the high spatial frequency. A lens was used to collimate the beam. An iris was put in front of the collimating lens to prevent the beam hitting the edge of the lens. Another iris (16 $\mathrm{cm}$ in diameter opening) was put after the collimating lens to select the size of the exposure area. With the photoresist side facing the laser beam, the slide was put on top of a rotation stage, right behind a 90 degree prism. 


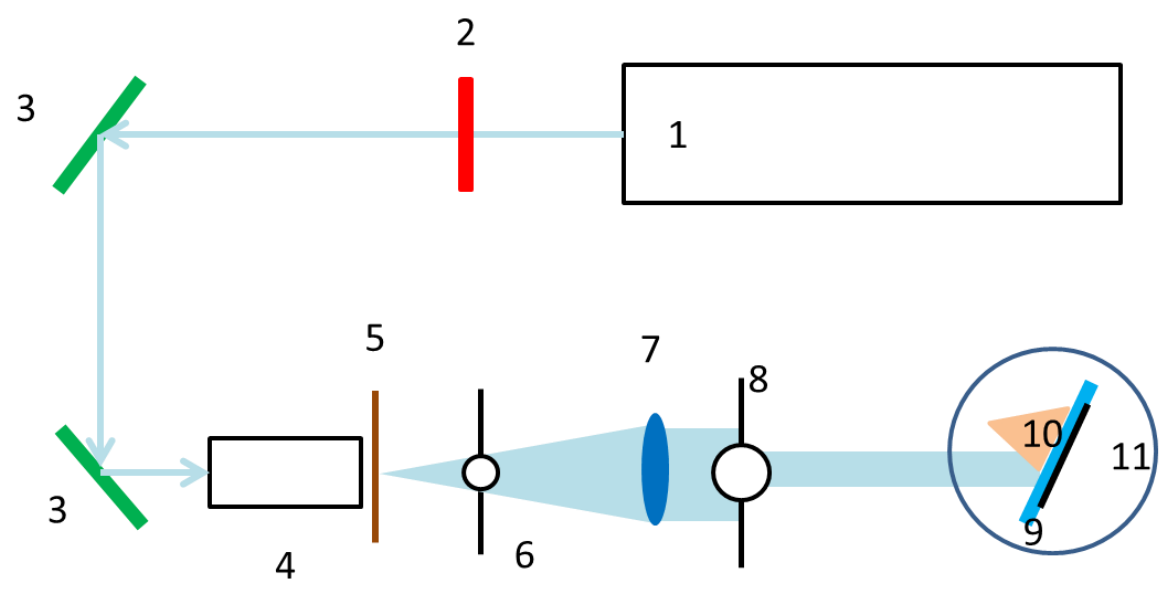

(a)

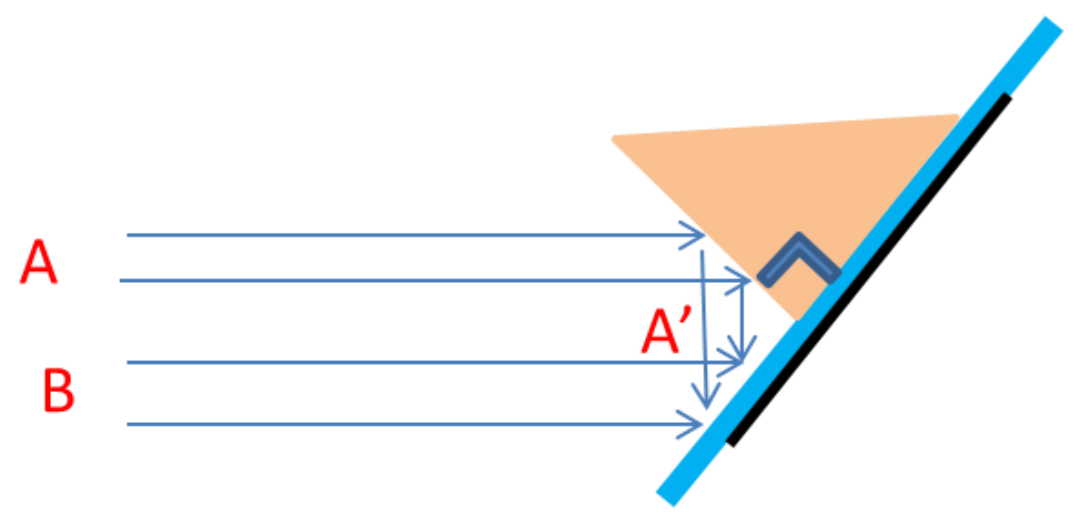

(b)

Appendix Figure 1: (a) Schematic figure of holography set-up for generation of the grating pattern and photoresist exposure. 1: He-Cd laser, 2: shutter, 3: mirror, 4: objective, 5: $10 \mu \mathrm{m}$ pin hole, 6: iris, 7: collimating lens, 8: iris, 9: photoresist coated glass slide with black paint covered back side, 10: right angle prism, and 11: rotation stage. (b) Interference pattern. Beam A' is the reflection of beam A from the prism surface. Beam A' interferes with beam B and generates interference pattern on photoresist layer. 
One half of the enlarged beam (A) hit the prism and was reflected by the surface $\left(A^{\prime}\right)$. The other half (B) hit the photoresist coated glass slide. Beam $A^{\prime}$ and beam B generate an interference pattern on the photoresist layer, as shown in Appendix Figure 1 (b). By rotating the stage, the interference pattern could be changed which modify the period of the grating. By changing the timer on the shutter, a suitable exposure time (approximately 5 seconds) was selected based on the power (approximately $2.4 \mathrm{~mW}$ ) of the laser beam after passing through all the optical elements. One side of the slide was exposed first, then the slide was flipped and the other side was exposed.

After the exposure, the slide was put into a beaker containing diluted 351 chemical developer solution (351 developer: DI water $=1: 4$ ) to develop the exposed photoresist. The beaker was put on top of a stirring plate with a mild stirring level. Littrow configuration was used to monitor the development process with a $543 \mathrm{~nm}$ incident laser beam, and a power meter was used to measure the - 1 order of transmission ( $\mathrm{T}_{-1}$ ) intensity [225] , as shown in Appendix Figure 2 (a). In Littrow configuration, the -1 order of reflection (R-1) was aligned with incident light. When $\mathrm{T}_{-1}$ was at maximum as shown in Appendix Figure 2 (b), the exposed photoresist was totally developed with an aspect ratio of 50:50. Then the slide was immediately taken out of the beaker, rinsed with DI water and blown dry with $\mathrm{N}_{2}$. 


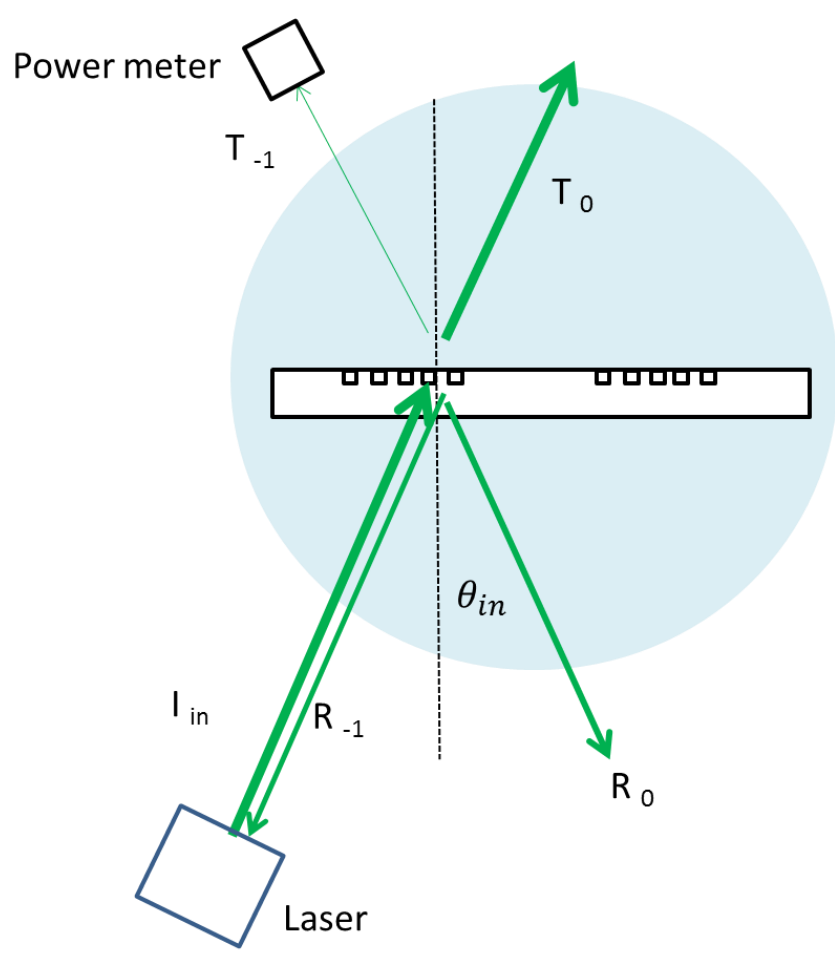

(a)

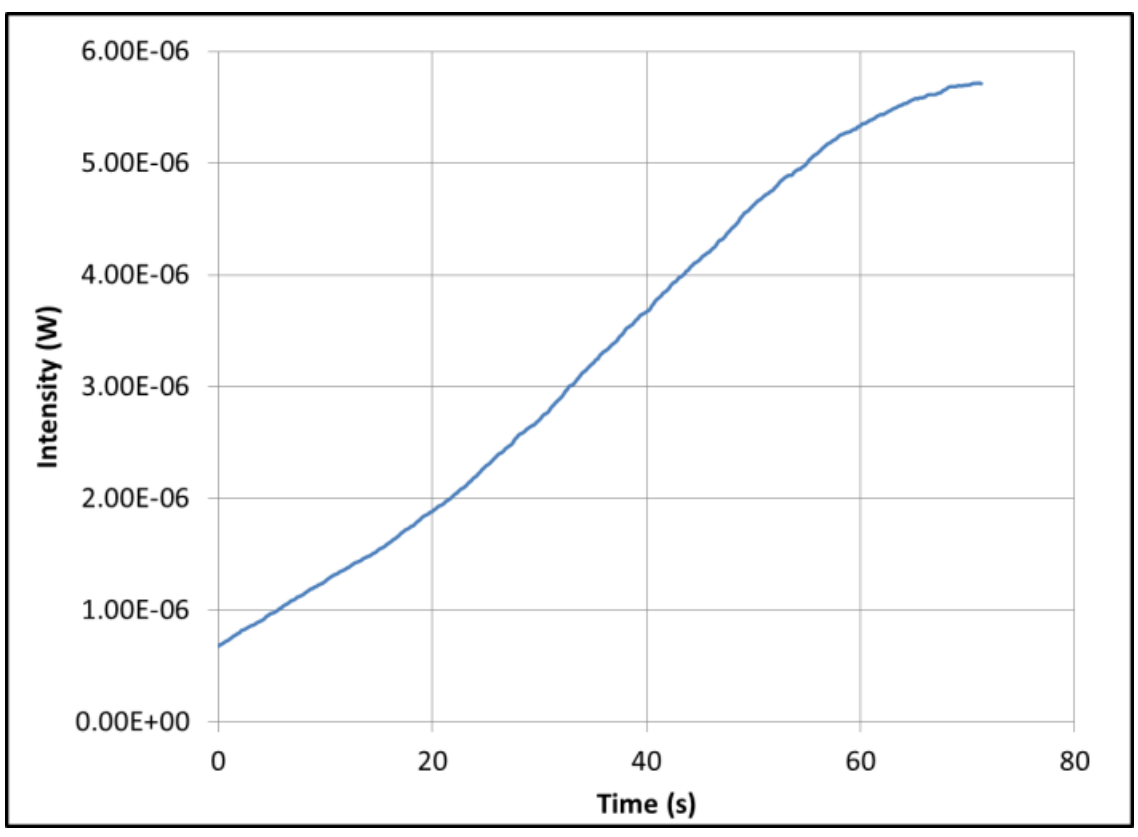

(b)

Appendix Figure 2: (a) Littrow configuration for the diffraction intensity monitoring in chemical development. (b) Intensity of the diffracted beam during a chemical developing process. 
Ion milling was used to transfer the grating pattern from the photoresist layer to the glass slide with 3 minutes of $\mathrm{CF}_{4}$ and 7 minutes of Ar plasma in sequence. The same cleaning a micro glass slide was used to remove undeveloped photoresist. SEM image of one grating sample was shown in Appendix Figure 3. The pitch size of this grating was $315 \mathrm{~nm}$ as measured.

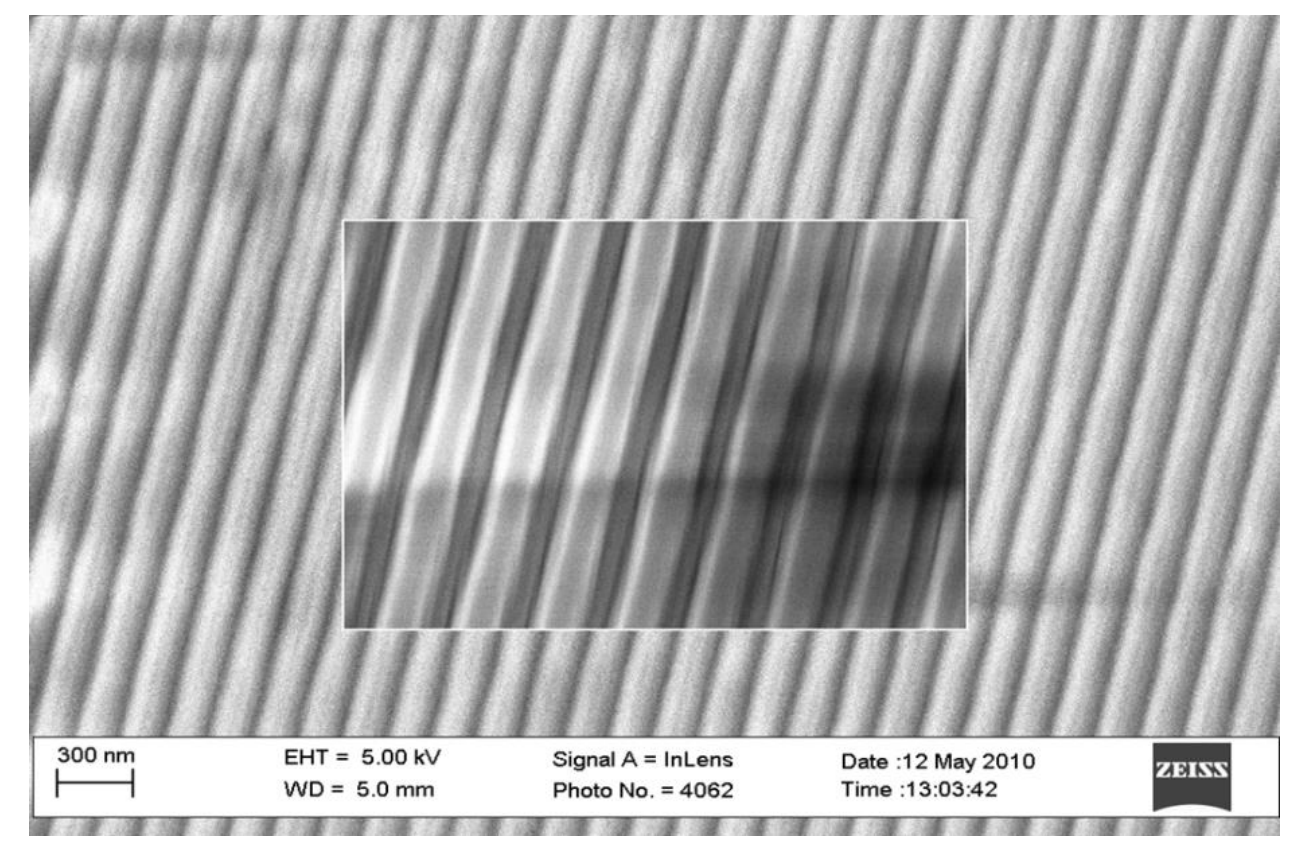

Appendix Figure 3: SEM image for a surface relief grating sample.

Before coating the aluminum oxide waveguide layer on top of the surface relief grating substrate, the slides were examined again for scratches and contamination that possibly generated during previous fabrication processes. The grating diffraction efficiency also was examined with Littrow configuration. The 
incident angle can be calculated with Equation Appendix 1-1 under the condition of $\theta_{\text {out }}=\theta_{\text {in }},-n_{\text {out }}=n_{\text {in }}=1$, and $m=-1$. For $543 \mathrm{~nm}$ incident light and $323 \mathrm{~nm}$ pitch size grating, $\theta_{i n}$ is 57.2 degree theoretically. With this incident angle, possible diffraction orders are only $0,-1$. The diffraction efficiency measurement is shown in Appendix Figure 4. A beam splitter is used to measure $R_{-1}$ beam indirectly through $R_{-1}^{\prime}$.

$$
\begin{aligned}
& n_{\text {out }} \cdot \sin \theta_{\text {out }}=n_{\text {in }} \cdot \sin \theta_{\text {in }}+m \frac{\lambda}{\Lambda} \\
& -2 n_{\text {in }} \cdot \sin \theta_{\text {in }}=-\frac{\lambda}{\Lambda}
\end{aligned}
$$

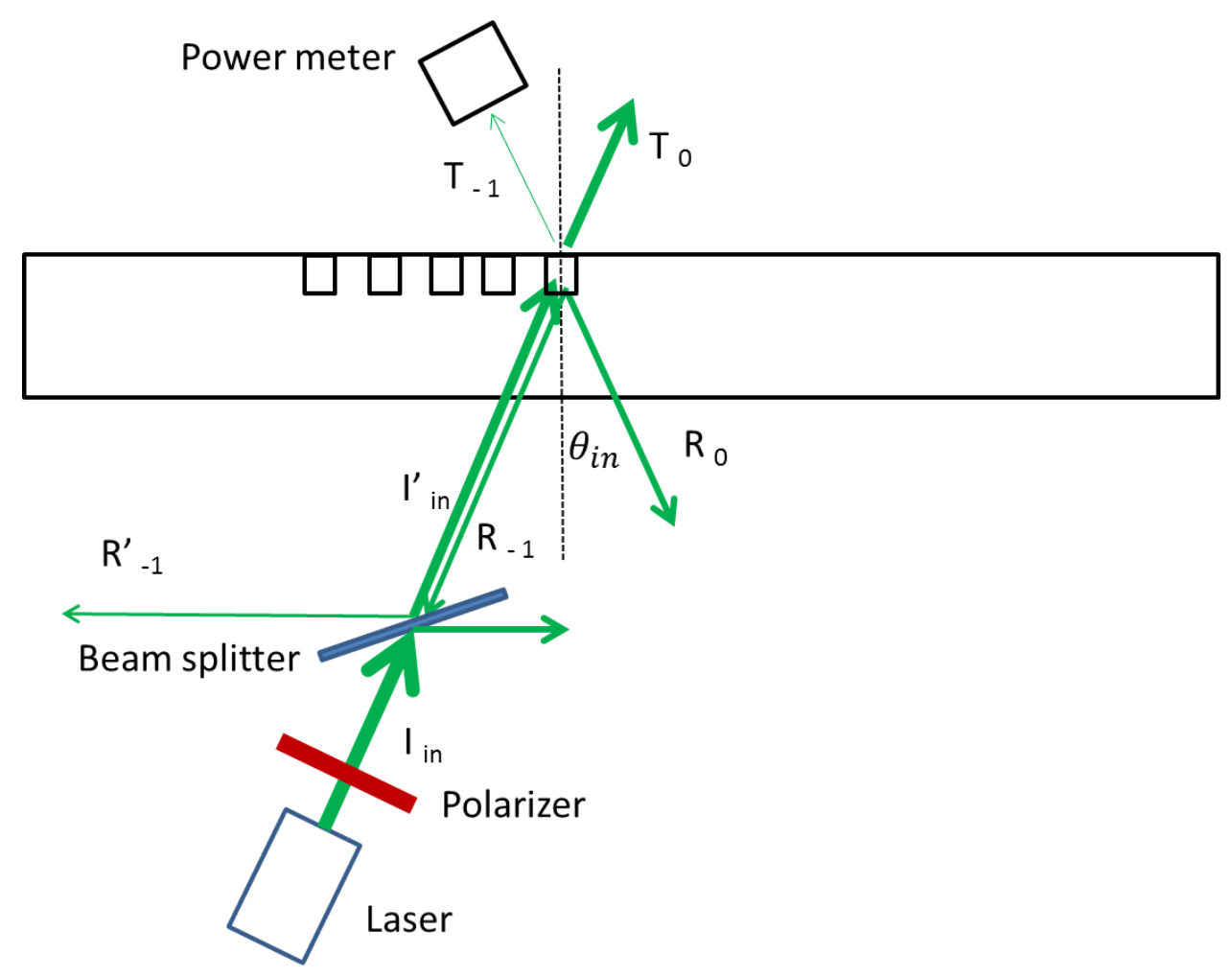

Appendix Figure 4: Littrow configuration for the diffraction efficiency measurement. 
In Appendix table 1, the measured diffraction efficiencies were shown for both left and right side gratings of one sample. The measured incident angles were close to the theoretical calculation. The summation of intensities of all the diffracted and reflected beams was close to the incident beam, and no other diffraction orders were observed.

Appendix table 1: Diffraction efficiency measurement

\begin{tabular}{|c|c|c|c|c|c|c|c|}
\hline & $I_{\text {in }}(\mu W)$ & $R_{0}(\mu W)$ & $T_{0}(\mu W)$ & $R_{-1}(\mu W)$ & $T_{-1}(\mu W)$ & $\begin{array}{c}\text { Total } \\
\text { percentage }\end{array}$ & $\theta_{\text {in }}$ \\
\hline $\begin{array}{c}\text { Left } \\
\text { grating }\end{array}$ & 1152.8 & 154.4 & 912 & $\begin{array}{l}36.61 \\
(3.2 \%)\end{array}$ & $\begin{array}{c}44.7 \\
(3.9 \%)\end{array}$ & $96.7 \%$ & $2.3^{\circ}$ \\
\hline $\begin{array}{l}\text { Right } \\
\text { grating }\end{array}$ & 1200 & 149.6 & 930.5 & $\begin{array}{c}52.12 \\
(4.3 \%)\end{array}$ & $\begin{array}{c}65.3 \\
(5.4 \%)\end{array}$ & $99.79 \%$ & $2.1^{\circ}$ \\
\hline
\end{tabular}


Appendix 3: Mathematica program based the transfer-matrix method for the transmittance calculation for a multi-layer structure

$$
\begin{aligned}
& \text { Clear }\left[\theta, \lambda, \mathrm{n}_{0}, \mathrm{n}_{m}, \mathrm{~N}_{e}, \mathrm{M}, \mathrm{V}_{s}, \mathrm{~V}_{p}, \mathrm{U}_{s}, \mathrm{U}_{p}\right] \\
& \theta=0 \text {; } \\
& \mathrm{M}=600 \text {; } \\
& \lambda_{i}=300 ; \lambda_{f}=900 \text {; } \\
& \mathrm{n}_{0}=1.00 \text {; } \\
& \mathrm{t}_{1}=7 ; \mathrm{t}_{2}=16 ; \mathrm{t}_{3}=409 \text {; } \\
& \mathrm{n}_{m}=1.51 \text {; } \\
& \mathrm{i}=0 \text {; } \\
& \text { Label[aa]; } \\
& \lambda=\lambda_{f}-\mathrm{i}\left(\lambda_{f}-\lambda_{i}\right) / \mathrm{M} \text {; } \\
& \mathrm{N}_{e}=\mathrm{n}_{0} \operatorname{Sin}[\pi \theta / 180] \text {; } \\
& \mathrm{n}_{1}=2.1-0.00011 \mathrm{I} \text {; } \\
& \mathrm{n}_{2}=1.46-0.00012 \mathrm{I} \text {; } \\
& \mathrm{n}_{3}=\left(1.64576+42.89898 / \lambda^{2}+308958233.142 / \lambda^{4}\right)-0.0 \mathrm{I} ; \\
& \chi_{0}=\operatorname{Abs}\left[\operatorname{Re}\left[\sqrt{n_{0}^{2}-N_{e}^{2}}\right]\right]-\operatorname{Abs}\left[\operatorname{Im}\left[\sqrt{n_{0}^{2}-N_{e}^{2}}\right]\right] \mathrm{I} ; \\
& s \eta_{0}=\chi_{0} ; \\
& \mathrm{p}_{0}=\mathrm{n}_{0}^{2} / \chi_{0} \text {; } \\
& \chi_{1}=\operatorname{Abs}\left[\operatorname{Re}\left[\sqrt{n_{1}^{2}-N_{e}^{2}}\right]\right]-\operatorname{Abs}\left[\operatorname{Im}\left[\sqrt{n_{1}^{2}-N_{e}^{2}}\right]\right] \mathrm{I} \text {; } \\
& s \eta_{1}=\chi_{1} \\
& \mathrm{p} \eta_{1}=\mathrm{n}_{1}^{2} / \chi_{1} \text {; } \\
& \delta_{1}=\frac{2 \pi}{\lambda} \mathrm{t}_{1} \chi_{1} \text {; } \\
& \chi_{2}=\operatorname{Abs}\left[\operatorname{Re}\left[\sqrt{n_{2}^{2}-N_{e}^{2}}\right]\right]-\operatorname{Abs}\left[\operatorname{Im}\left[\sqrt{n_{2}^{2}-N_{e}^{2}}\right]\right] \mathrm{I} \text {; } \\
& s \eta_{2}=\chi_{2} ; \\
& \mathrm{p} \eta_{2}=\mathrm{n}_{2}^{2} / \chi_{2} \text {; } \\
& \delta_{2}=\frac{2 \pi}{\lambda} \mathrm{t}_{2} \chi_{2} \text {; } \\
& \chi_{3}=\operatorname{Abs}\left[\operatorname{Re}\left[\sqrt{n_{3}^{2}-N_{e}^{2}}\right]\right]-\operatorname{Abs}\left[\operatorname{Im}\left[\sqrt{n_{3}^{2}-N_{e}^{2}}\right]\right] \mathrm{I} \text {; } \\
& s \eta_{3}=\chi_{3} ;
\end{aligned}
$$




$$
\begin{aligned}
& \mathrm{p \eta}_{3}=\mathrm{n}_{3}^{2} / \chi_{3} \\
& \delta_{3}=\frac{2 \pi}{\lambda} \mathrm{t}_{3} \chi_{3} \text {; } \\
& \chi_{m}=\operatorname{Abs}\left[\operatorname{Re}\left[\sqrt{n_{m}^{2}-N_{e}^{2}}\right]\right]-\operatorname{Abs}\left[\operatorname{Im}\left[\sqrt{n_{m}^{2}-N_{e}^{2}}\right]\right] \mathrm{I} \text {; } \\
& s \eta_{m}=\chi_{m} ; \\
& \mathrm{p \eta}_{m}=\mathrm{n}_{m}^{2} / \chi_{m} \\
& M_{s}=\left(\begin{array}{cc}
\operatorname{Cos}\left[\delta_{1}\right] & \mathrm{I} \frac{\operatorname{Sin}\left[\delta_{1}\right]}{\mathrm{s} \eta_{1}} \\
\mathrm{I} \mathrm{s} \eta_{1} \operatorname{Sin}\left[\delta_{1}\right] & \operatorname{Cos}\left[\delta_{1}\right]
\end{array}\right)\left(\begin{array}{cc}
\operatorname{Cos}\left[\delta_{2}\right] & \mathrm{I} \frac{\operatorname{Sin}\left[\delta_{2}\right]}{\mathrm{s} \eta_{2}} \\
\mathrm{I} \operatorname{s\eta }_{2} \operatorname{Sin}\left[\delta_{2}\right] & \operatorname{Cos}\left[\delta_{2}\right]
\end{array}\right) \\
& \left(\begin{array}{cc}
\operatorname{Cos}\left[\delta_{3}\right] & \mathrm{I} \operatorname{Sin}\left[\delta_{3}\right] / s \eta_{2} \\
\mathrm{I} \eta_{2} \operatorname{Sin}\left[\delta_{3}\right] & \operatorname{Cos}\left[\delta_{3}\right]
\end{array}\right) \text {; } \\
& M_{p}=\left(\begin{array}{cc}
\operatorname{Cos}\left[\delta_{1}\right] & \mathrm{I} \frac{\operatorname{Sin}\left[\delta_{1}\right]}{\mathrm{p} \eta_{1}} \\
\mathrm{I} \eta_{1} \operatorname{Sin}\left[\delta_{1}\right] & \operatorname{Cos}\left[\delta_{1}\right]
\end{array}\right)\left(\begin{array}{cc}
\operatorname{Cos}\left[\delta_{2}\right] & \mathrm{I} \frac{\operatorname{Sin}\left[\delta_{2}\right]}{\mathrm{p} \eta_{2}} \\
\mathrm{I} \eta_{2} \operatorname{Sin}\left[\delta_{2}\right] & \operatorname{Cos}\left[\delta_{2}\right]
\end{array}\right) \\
& \left(\begin{array}{cc}
\operatorname{Cos}\left[\delta_{3}\right] & \mathrm{I} \operatorname{Sin}\left[\delta_{3}\right] / \mathrm{p \eta}_{2} \\
\mathrm{I} \mathrm{p} \eta_{2} \operatorname{Sin}\left[\delta_{3}\right] & \operatorname{Cos}\left[\delta_{3}\right]
\end{array}\right) \text {; } \\
& V_{s}=\left(\begin{array}{c}
1 \\
s \eta_{m}
\end{array}\right) \text {; } \\
& U_{s}=M_{s} V_{s} \\
& V_{p}=\left(\begin{array}{c}
1 \\
\mathrm{p} \eta_{m}
\end{array}\right) \text {; } \\
& U_{p}=M_{p} V_{p} \\
& B_{s}=U_{s}[1,1] \\
& C_{s}=U_{s}[2,1] \text {; } \\
& B_{p}=U_{p}[1,1] \\
& C_{p}=U_{p}[2,1] \\
& R_{S}=\operatorname{Re}\left[\frac{\mathrm{s} \eta_{0} B_{S}-C_{S}}{s \eta_{0} B_{S}+C_{S}} \text { conjugate }\left(\frac{\mathrm{s} \eta_{0} B_{S}-C_{S}}{s \eta_{0} B_{S}+C_{S}}\right)\right] ; \\
& T_{S}=\operatorname{Re}\left[\frac{4 \mathrm{~s} \eta_{0} R e\left[s \eta_{m}\right]}{s \eta_{0} B_{S}+C_{S}} \text { conjugate }\left(\frac{1}{s \eta_{0} B_{S}+C_{S}}\right)\right] ; \\
& A_{s}=\operatorname{Re}\left[\frac{4 \mathrm{~s} \eta_{0} R e\left[B_{S} * \text { conjugate }\left[C_{S}\right]-s \eta_{m}\right]}{s \eta_{0} B_{S}+C_{S}} \text { conjugate }\left(\frac{1}{s \eta_{0} B_{S}+C_{S}}\right)\right] ; \\
& R_{p}=\operatorname{Re}\left[\frac{\mathrm{p} \eta_{0} B_{p}-C_{p}}{\mathrm{p}_{0} B_{p}+C_{p}} \text { conjugate }\left(\frac{\mathrm{p} \eta_{0} B_{p}-C_{p}}{\mathrm{p} \eta_{0} B_{p}+C_{p}}\right)\right] ; \\
& T_{p}=\operatorname{Re}\left[\frac{4 \eta_{0} R e\left[\mathrm{p}_{m}\right]}{\mathrm{p \eta}_{0} B_{p}+C_{p}} \text { conjugate }\left(\frac{1}{\mathrm{p}_{0} B_{p}+C_{p}}\right)\right] ;
\end{aligned}
$$




$$
\begin{aligned}
& A_{p}=\operatorname{Re}\left[\frac{4 \mathrm{p} \eta_{0} R e\left[B_{p} * \text { conjugate }\left[C_{p}\right]-s \eta_{m}\right]}{\mathrm{p}_{0} B_{p}+C_{p}} \text { conjugate }\left(\frac{1}{\mathrm{p}_{0} B_{p}+C_{p}}\right)\right] ; \\
& \text { Lambda }[\mathrm{i}]=\lambda \text {; } \\
& T_{s}[\mathrm{i}]=T_{s} \text {; } \\
& T_{p}[\mathrm{i}]=T_{p} ; \\
& \operatorname{Print}[\lambda] \text {; } \\
& \operatorname{Print}\left[\frac{4 \mathrm{n}_{m} \mathrm{n}_{0}}{\left(\mathrm{n}_{m}+\mathrm{n}_{0}\right)^{2}} T_{S}\right] \text {; } \\
& \mathrm{i}+=1 \text {; } \\
& \text { If }[\mathrm{i}<(\mathrm{M}+1) \text {, Goto[aa]]; } \\
& \text { saf0 }=\text { Table }\left[\left\{\text { Lambda }[\mathrm{i}], \frac{4 \mathrm{n}_{m} \mathrm{n}_{0}}{\left(\mathrm{n}_{m}+\mathrm{n}_{0}\right)^{2}} T_{s}[\mathrm{i}]\right\},\{\mathrm{i}, 0, \mathrm{M}, 1\}\right] ; \\
& \text { Export["saf0.xls",saf0]; }
\end{aligned}
$$


Appendix 4: Normalized transmittance curves for TE and TM polarization incident light, and for different thicknesses of ultra-thin ITO films under CV potential scan

The spectroscopic features of the ultra-thin ITO layers under the CV potential scans in neutral phosphate buffer were examined for different thicknesses films and different polarized incident light.

Both $13 \mathrm{~nm}$ and $8 \mathrm{~nm}$ ultra-thin ITO films were tested, and the calculated normalized transmittance profiles were plotted for different laser beams as shown in Appendix Figure 5. For the CV potential scan, a range from $+0.8 \mathrm{~V}$ to $-0.4 \mathrm{~V}$ was used with $0.02 \mathrm{~V} / \mathrm{s}$ speed. The internal trigger for the ICCD camera was set as $2 \mathrm{~Hz}$. The exposure time for each frame was $200 \mathrm{~ms}$. No strong difference could be observed for $13 \mathrm{~nm}$ and $8 \mathrm{~nm}$ ITO film under the same CV potential scan. The normalized curve was shifted by one unit to show the difference. 


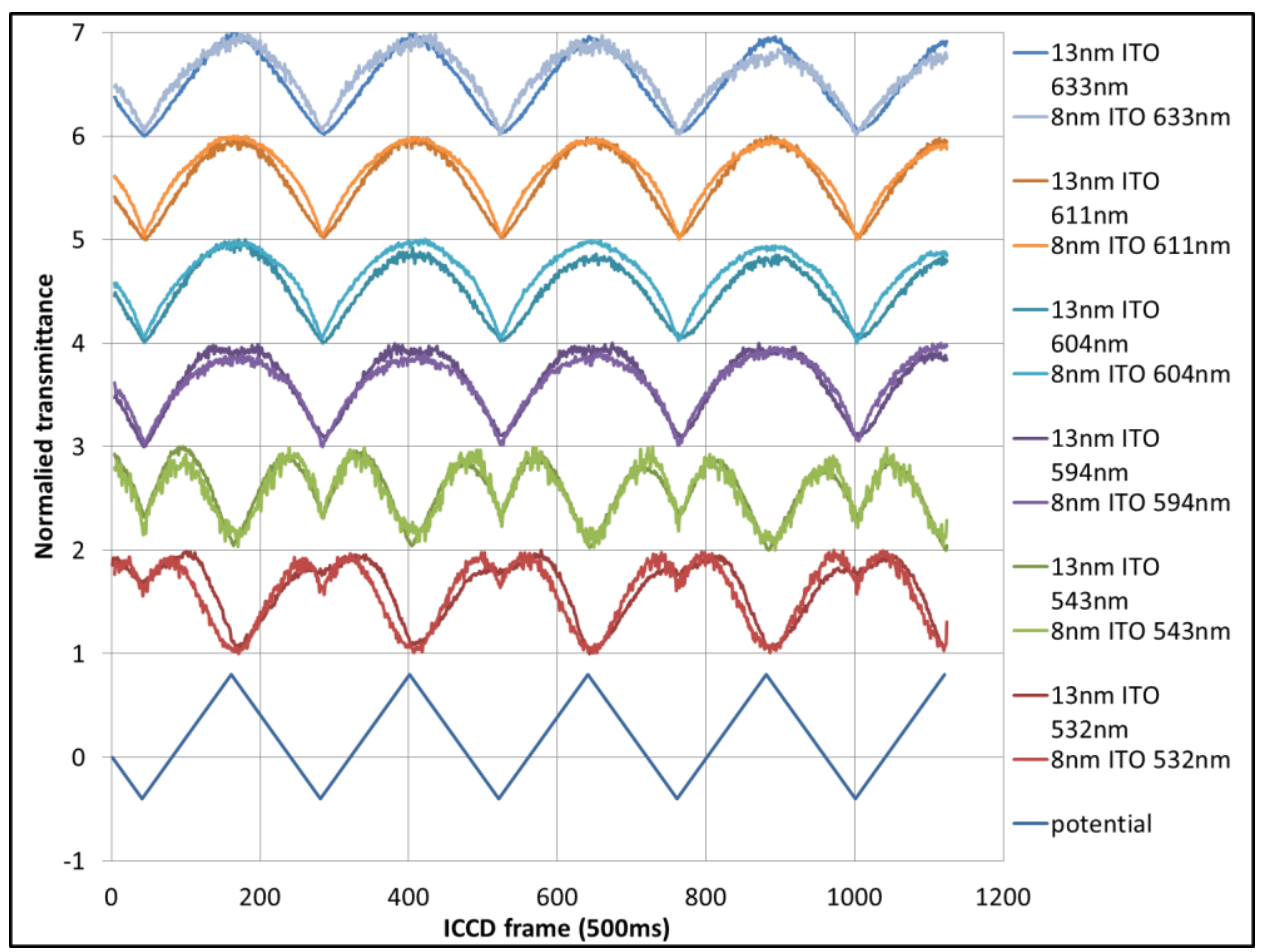

Appendix Figure 5: Normalized transmittance profiles for $13 \mathrm{~nm}$ and $8 \mathrm{~nm}$ ITO films under the same $\mathrm{CV}$ potential scan. The potential modulation is plotted.

Both TE and TM polarize incident light were used to test a $13 \mathrm{~nm}$ ultra-thin ITO films, and the calculated normalized transmittance profiles were plotted for different laser beams as shown in Appendix Figure 6. The same CV potential scans were used for each laser beam and there was no difference could be observed for the two polarized incident light. The normalized curve was shifted by one unit to show the difference. 


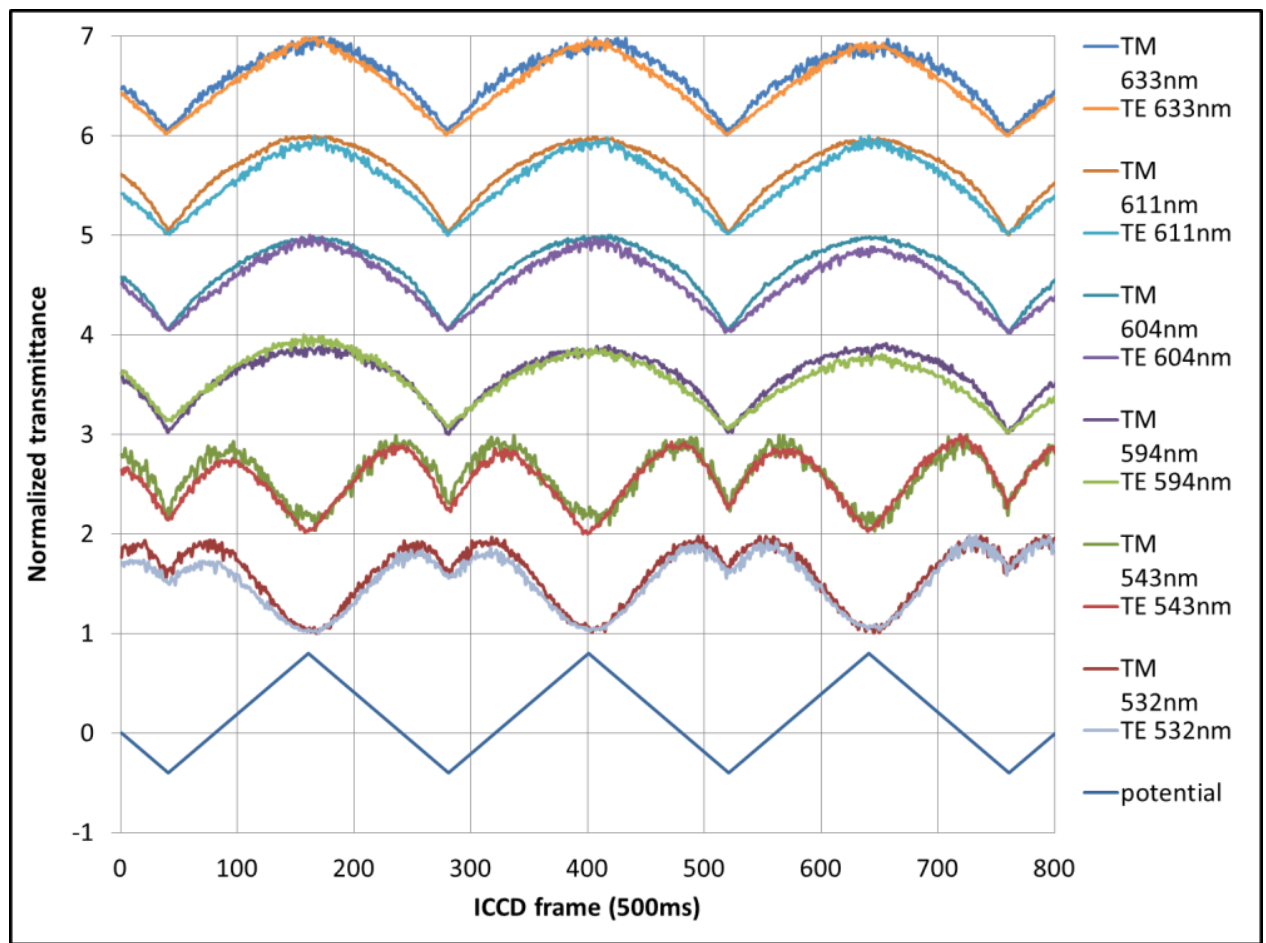

Appendix Figure 6: Normalized transmittance profiles with different polarized incident light for a $13 \mathrm{~nm}$ ITO film under the CV potential scan.

The similar spectroscopic features of ultra-thin ITO films under the same CV potential scan were observed among cases of different thickness of ITO layer and different polarized incident light. 
Appendix 5: Out-coupled intensity against the incident angle profiles for the ultrathin ITO film under the constant potential step from different probing laser beams

The spectroscopic features of the ultra-thin ITO layers under the constant potential step in a neutral phosphate buffer were examined with different laser beams. In Appendix Figure 7, the results of out-coupled intensity against incident angle profiles from $502 \mathrm{~nm}, 543 \mathrm{~nm}$ and $633 \mathrm{~nm}$ were shown along with the comparison to the $\mathrm{CV}$ potential scan results in normalized transmittance. From all these three laser beams, the same phenomenon as $514 \mathrm{~nm}$ and $594 \mathrm{~nm}$ laser beams was observed. The coupling angle did not shift severely between the potential steps. The absorbance oscillations between the potential steps were dominant, and the oscillations trend was decided by the probing wavelength.

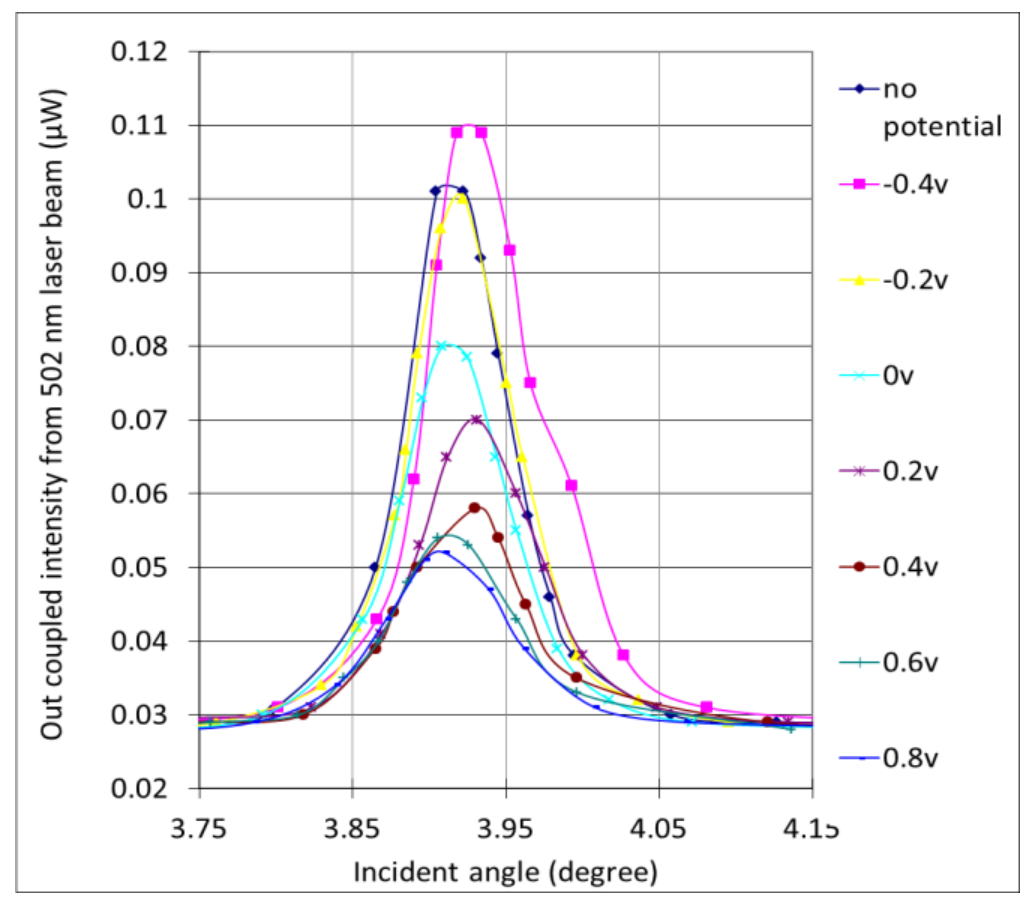




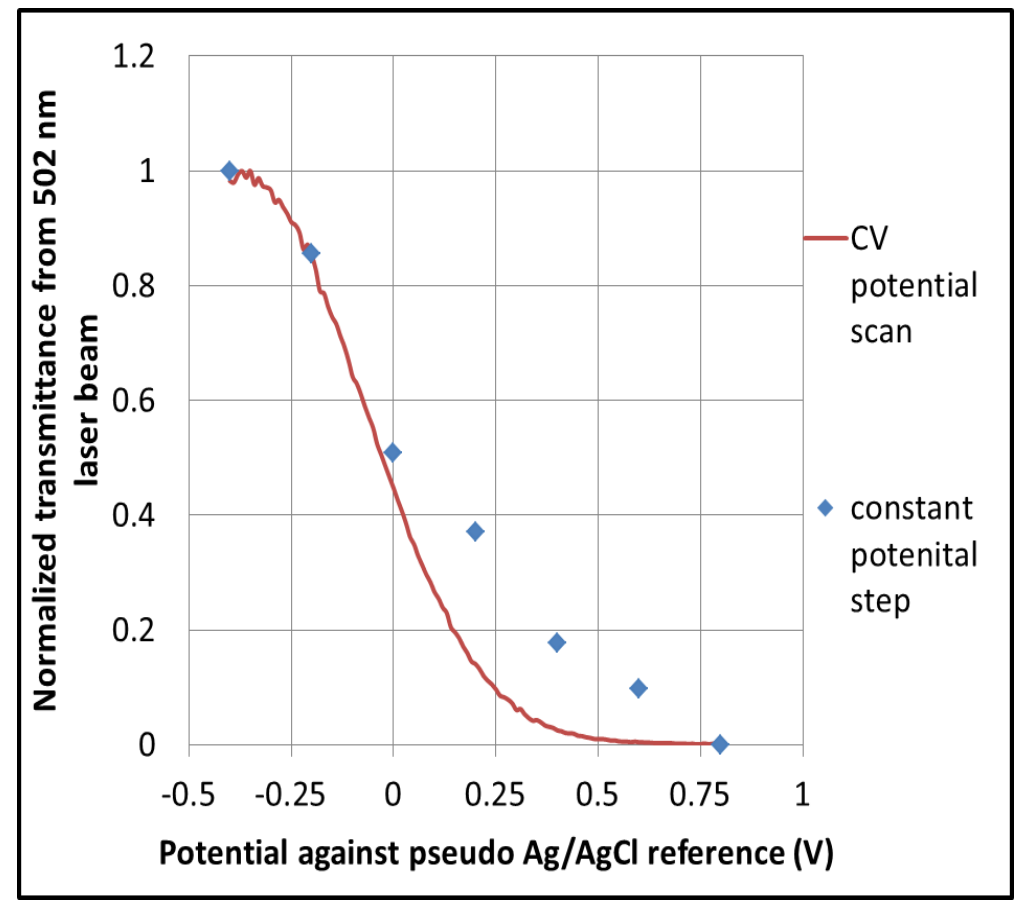

(a)

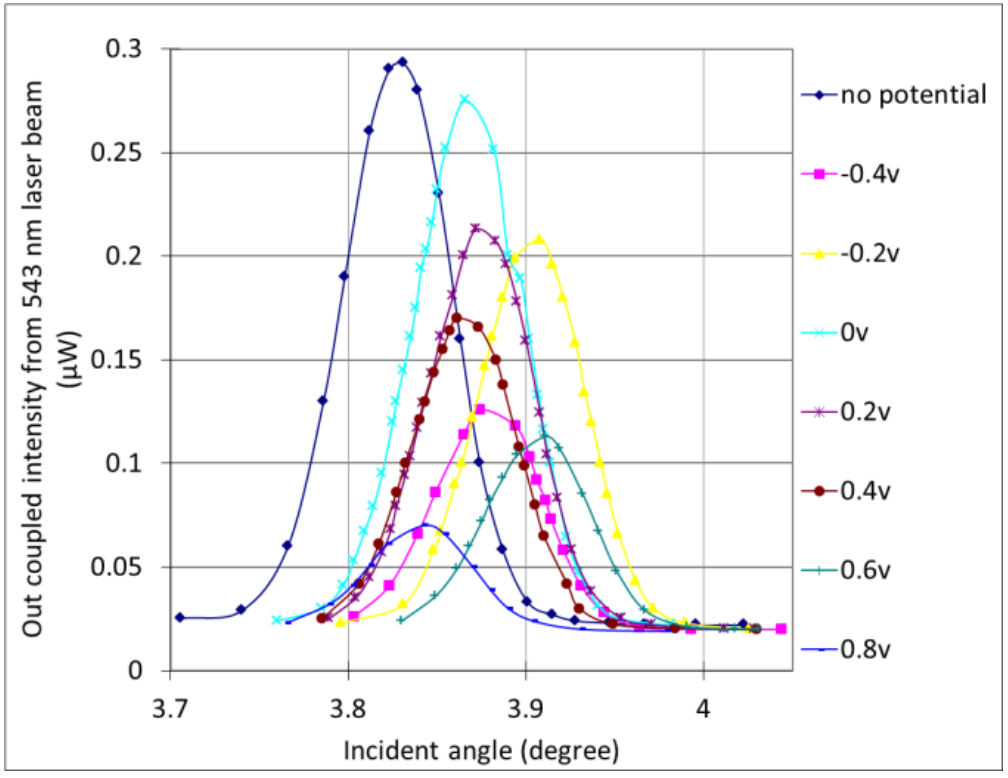




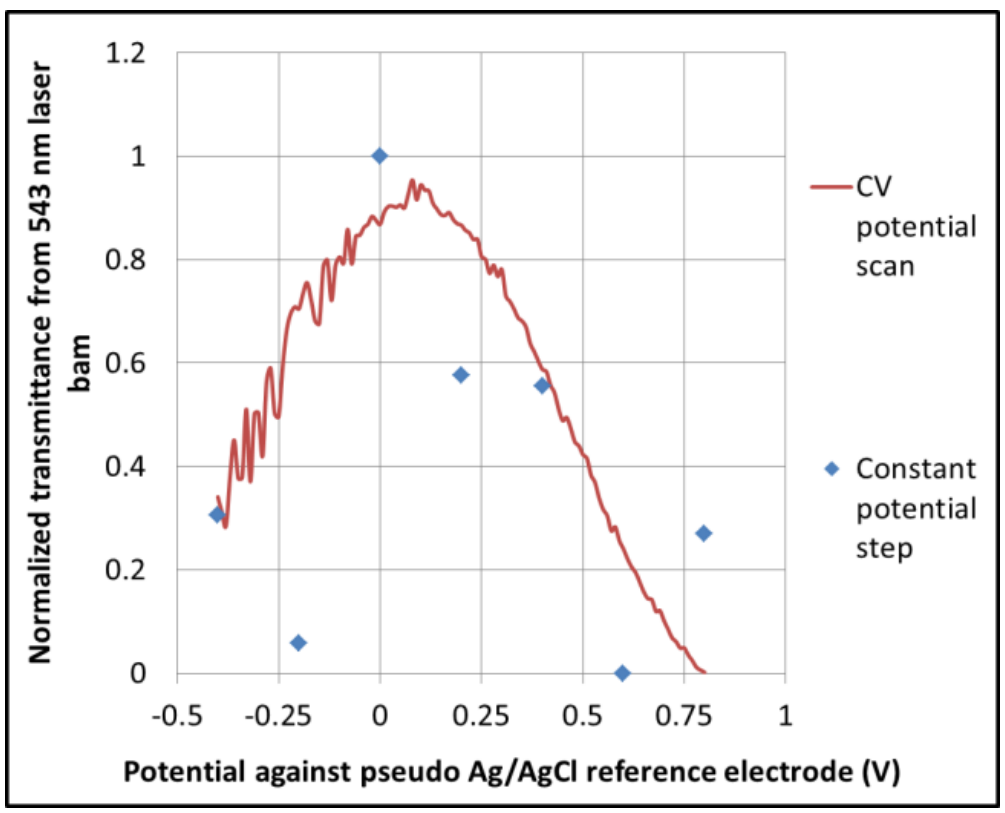

(b)

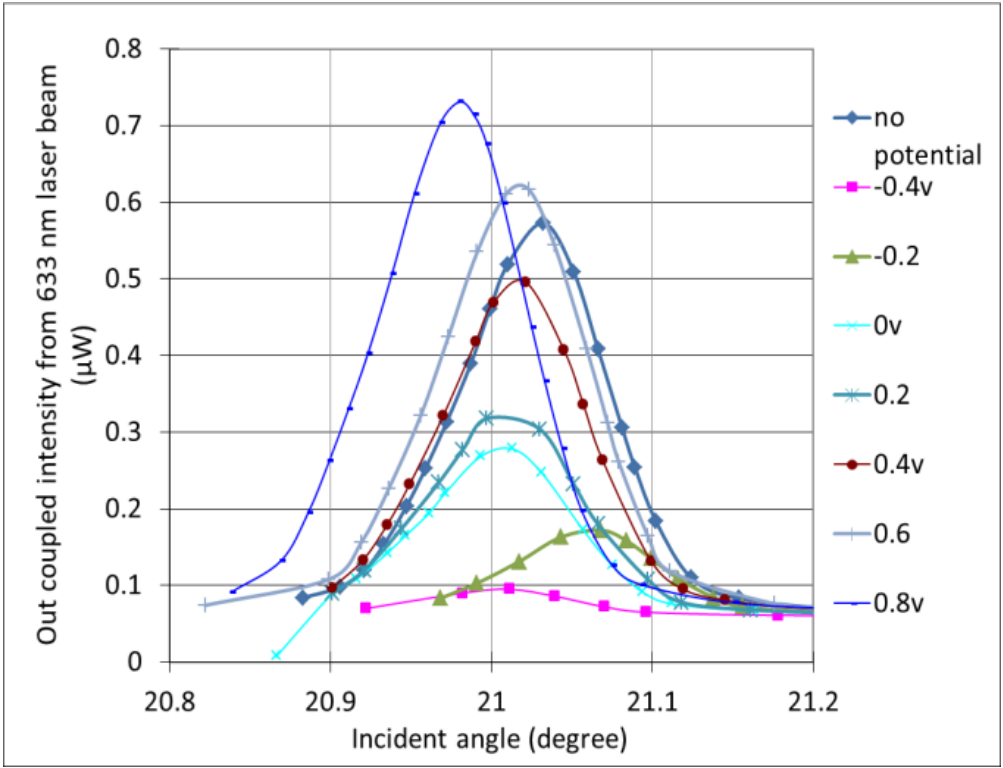




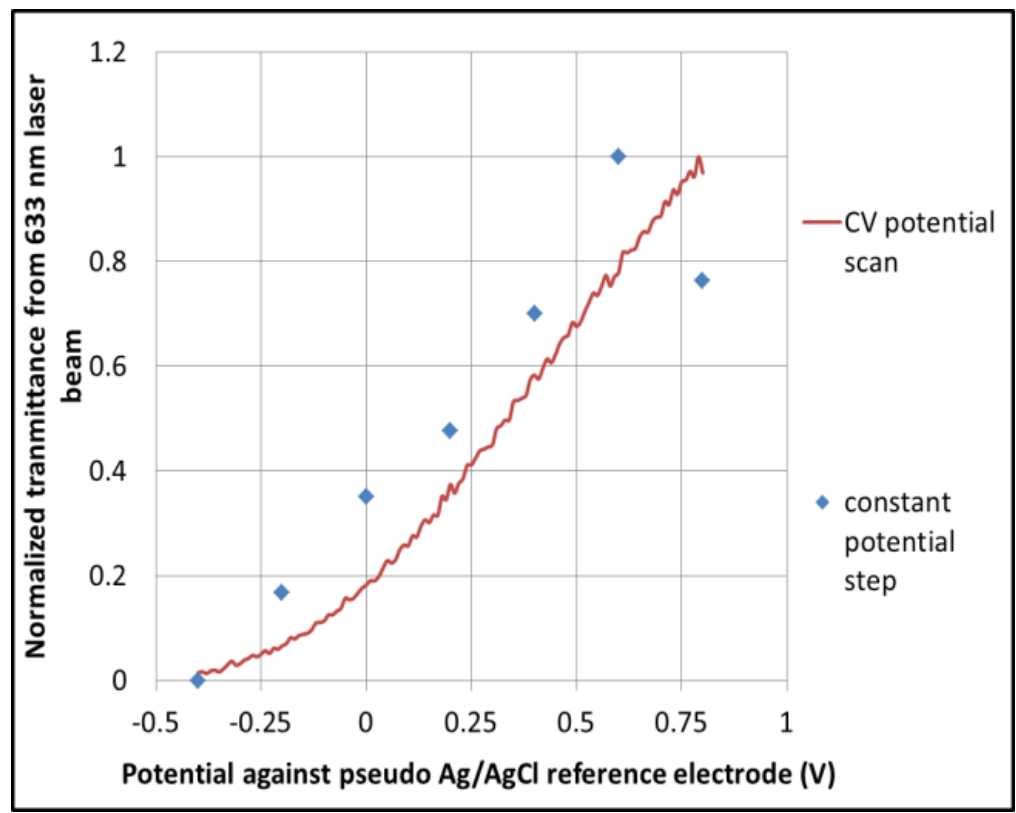

(c)

Appendix Figure 7: The out-coupled intensity against incident angle is shown as the top graph, and the comparison of the normalized transparence between the constant potential step and the CV potential scan is shown on the bottom side graph for each laser beam (a) $502 \mathrm{~nm}$, (b) $543 \mathrm{~nm}$ and (c) $633 \mathrm{~nm}$. 
Appendix 6: Examination of the ultra-thin ITO film under AC impedance potential modulation with TE and TM polarized light

The spectroscopic features of the ultra-thin ITO layers under the AC impedance potential modulation were examined with both TE and TM polarized incident light. In Appendix Figure 8, the ratios of the real AC component over the DC component and the ratios of the imaginary $\mathrm{AC}$ component over the $\mathrm{DC}$ component were plotted in the format of complex planes for both polarization incident lights. The potential modulation frequency increased in the clockwise direction. No difference could be observed.

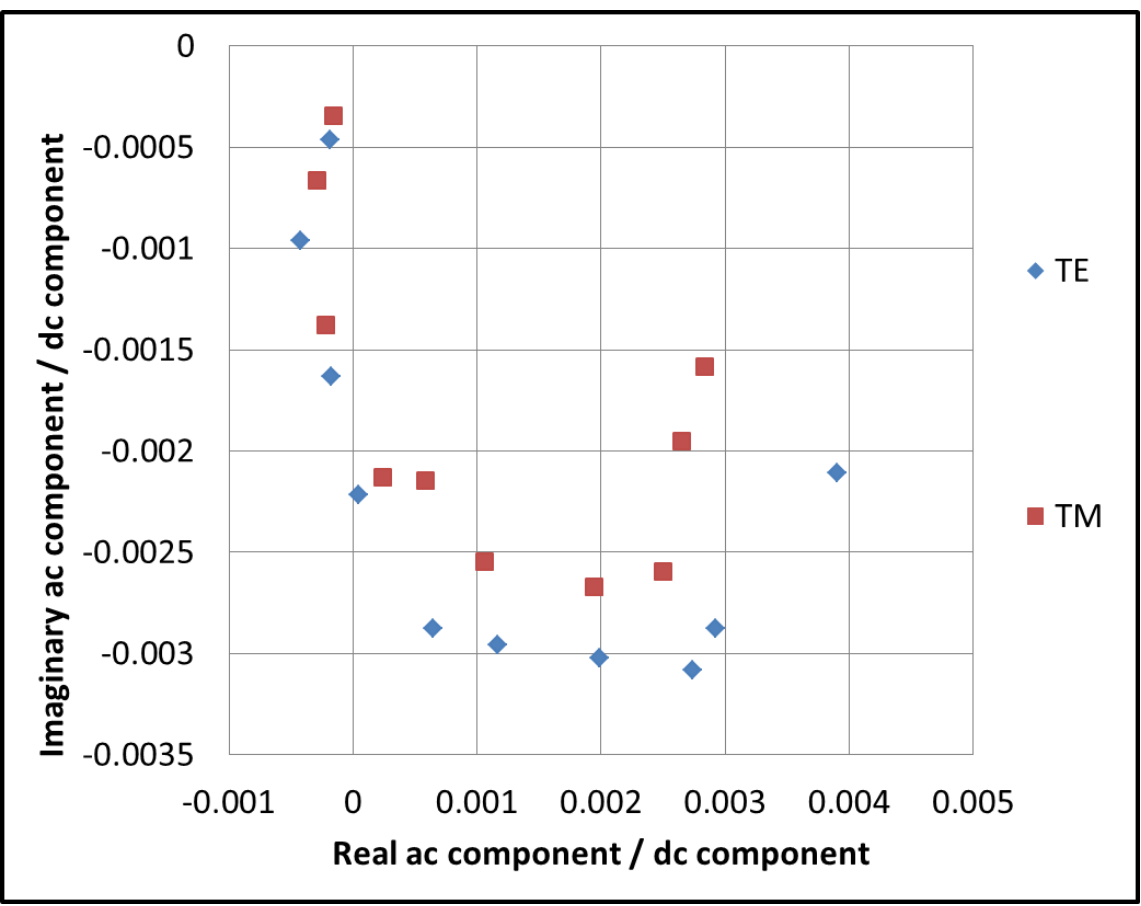

Appendix Figure 8: Ratio of AC component over DC component in complex planes for both polarized incident lights. 
Appendix 7: Potential different calibration between the homemade pseudo $\mathrm{Ag} / \mathrm{AgCl}$ reference electrode and the standard $\mathrm{Ag} / \mathrm{AgCl}$ reference electrode

A homemade pseudo $\mathrm{Ag} / \mathrm{AgCl}$ wire was used as a reference electrode, as mentioned in Chapter 3. For the purpose to obtain the accurate information of the formal potential for a redox reaction, the potential difference of the pseudo reference electrode in a buffer solution $\left(5 \mathrm{mM} \mathrm{NaH}_{2} \mathrm{PO}_{4}\right)$ was calibrated with the standard $\mathrm{Ag} / \mathrm{AgCl}$ reference electrode $(\mathrm{CHI})$ in a $1 \mathrm{M} \mathrm{KCl}$ solution. The standard $\mathrm{Ag} / \mathrm{AgCl}$ electrode has a potential of $+0.197 \mathrm{~V}$ difference when compared to the standard hydrogen reference electrode.

First, a directly potential difference was measured by a potential-meter between the pseudo $\mathrm{Ag} / \mathrm{AgCl}$ reference electrode and the standard $\mathrm{Ag} / \mathrm{AgCl}$ reference electrode. With the standard electrode connected to the positive side, and the pseudo electrode connected to the negative side, the potential difference was $0.0793 \mathrm{~V}$.

Second, the open circuit potentials were compared with a three-electrode system. An EA-SM-IOW device was used as a working electrode, and a Pt wire was used as the counter electrode. Different working electrodes (EA-SM-IOW devices) were used. The average of the potential difference between the standard and the pseudo $\mathrm{Ag} / \mathrm{AgCl}$ reference electrodes was - $0.0828 \mathrm{~V}$.

The third test was carried out in comparing cytochrome c protein redox reaction formal potentials under a $\mathrm{CV}$ potential scans with $0.2 \mathrm{~V} / \mathrm{s}$ speed from $-0.4 \mathrm{~V}$ 
to $0.4 \mathrm{~V}$. An extremely high bulk concentration $(100 \mu \mathrm{M})$ of original oxidized cytochrome c was tested. As shown in Appendix Figure 9, the red current curve was from using the standard reference electrode, and the formal potential of the redox reaction was $0.0420 \mathrm{~V}$ by taking the middle point of two faradaic current peak potentials. And it was close to the reference results for cytochrome c protein in a solution environment under the same condition. The blue current curve was from using the pseudo reference electrode, and the formal potential was $-0.0515 \mathrm{~V}$. The difference was $-0.0935 \mathrm{~V}$. The purpose of using this high bulk concentration of cytochrome $\mathrm{c}$ and this high potential scan speed was to make the electrical faradaic current peaks easily identified.

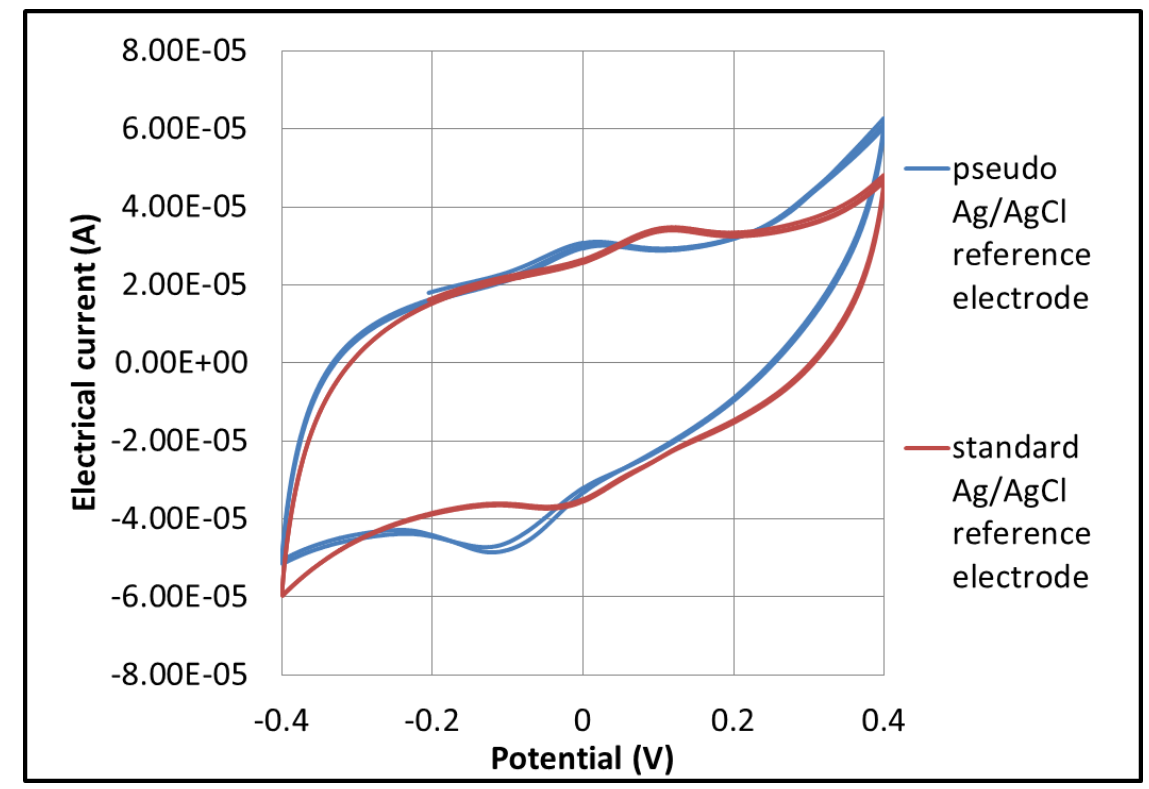

Appendix Figure 9: The formal potential of the redox reaction from high bulk concentration of cytochrome c proteins. 
Taking all three tests into account, the average of the potential difference between the pseudo and the standard $\mathrm{Ag} / \mathrm{AgCl}$ reference electrode is $-0.0852 \mathrm{~V}$. 
Appendix 8: Examination of cytochrome c proteins redox reaction under different AC potential amplitude

Different amplitudes of AC impedance modulations were used to test the EASM-IOW working electrode performance. $5 \mathrm{mV}, 10 \mathrm{mV}$, and $15 \mathrm{mV}$ were tested. The active surface coverage was plotted against the angular frequency at - $0.1 \mathrm{~V} \mathrm{DC}$ bias, as shown in Appendix Figure 10. As expected, the lower AC amplitude, the fewer cytochrome c proteins under the redox reaction were detected. The relationship of the active surface coverage and the AC potential amplitude was examined by selecting data from the slowest oscillation angular frequency, as shown in the inset. A linear fit was used. The active surface coverage from the $15 \mathrm{mV} \mathrm{AC}$ potential amplitude was in the linear range. 


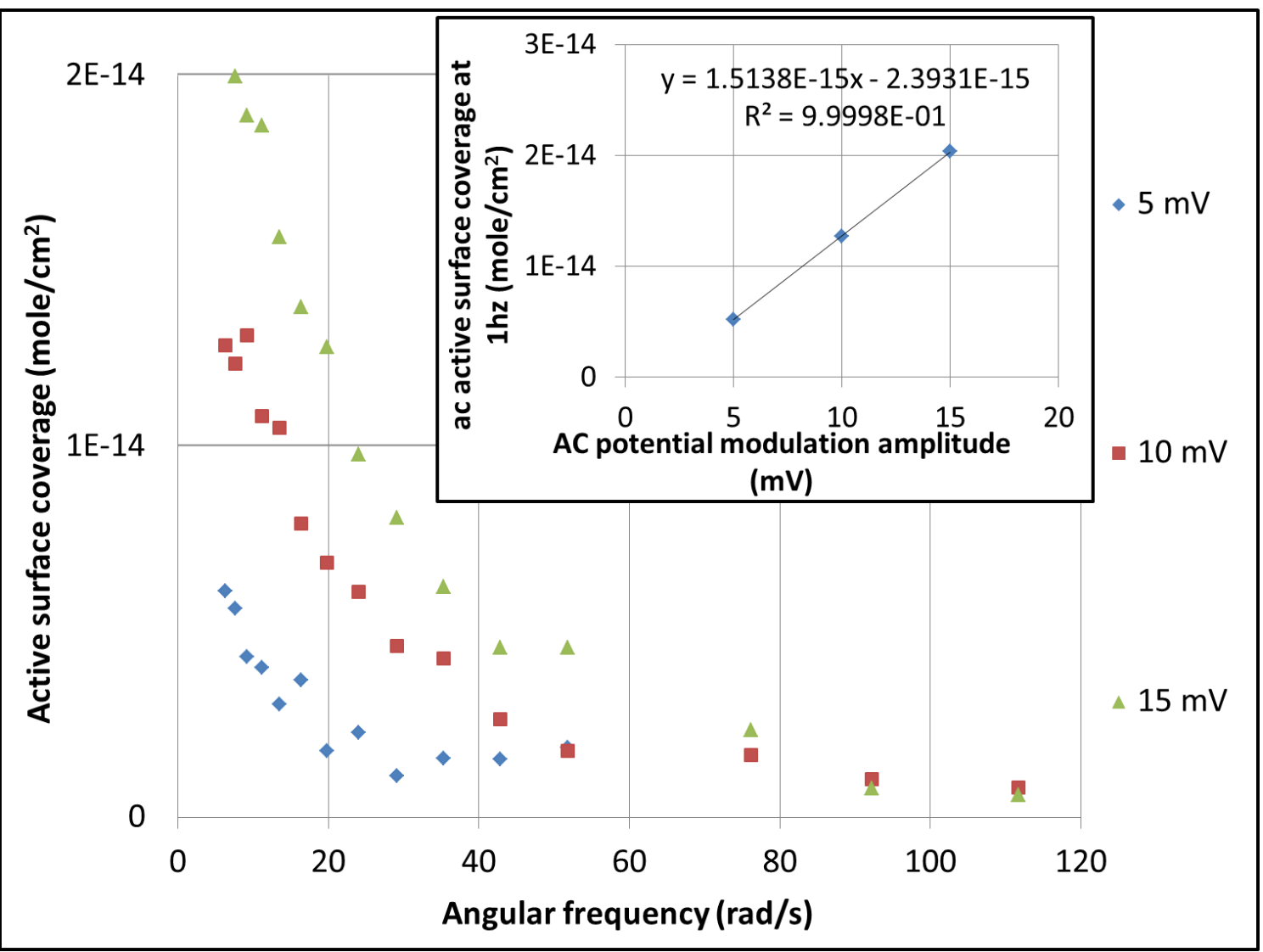

Appendix Figure 10: Optical impedance results of active surface coverage under different AC potential amplitude. A linear fit of the surface coverage and AC potential amplitude is shown in the inset. 
Appendix 9: Mathematica program for the SPR and the CPWR reflectivity simulation

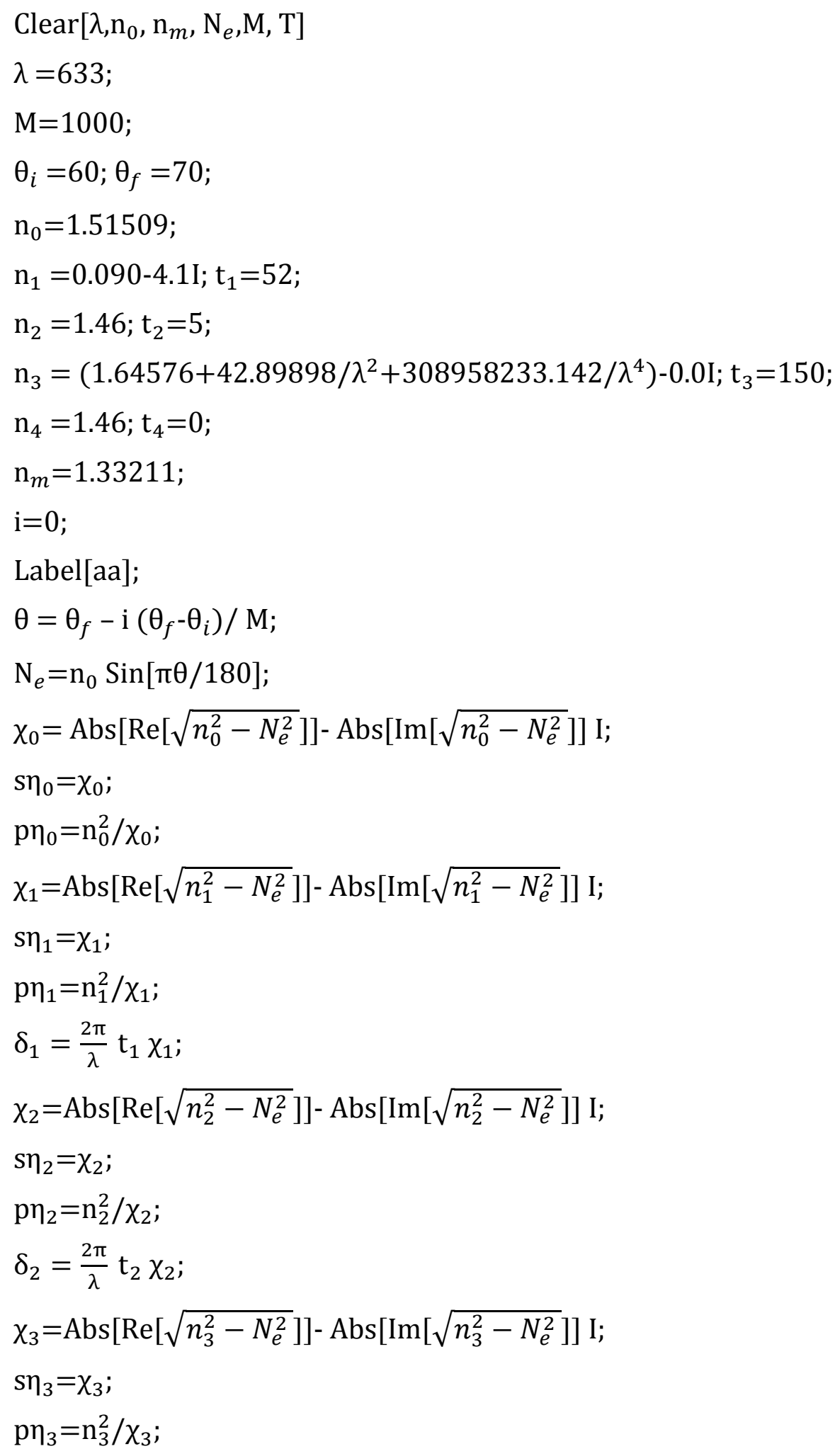




$$
\begin{aligned}
& \delta_{3}=\frac{2 \pi}{\lambda} \mathrm{t}_{3} \chi_{3} ; \\
& \chi_{4}=\operatorname{Abs}\left[\operatorname{Re}\left[\sqrt{n_{4}^{2}-N_{e}^{2}}\right]\right]-\operatorname{Abs}\left[\operatorname{Im}\left[\sqrt{n_{4}^{2}-N_{e}^{2}}\right]\right] \mathrm{I} \text {; } \\
& \mathrm{s \eta}_{4}=\chi_{4} ; \\
& \mathrm{p \eta}_{4}=\mathrm{n}_{4}^{2} / \chi_{4} \text {; } \\
& \delta_{4}=\frac{2 \pi}{\lambda} \mathrm{t}_{4} \chi_{4} \\
& \chi_{m}=\operatorname{Abs}\left[\operatorname{Re}\left[\sqrt{n_{m}^{2}-N_{e}^{2}}\right]\right]-\operatorname{Abs}\left[\operatorname{Im}\left[\sqrt{n_{m}^{2}-N_{e}^{2}}\right]\right] \mathrm{I} ; \\
& \mathrm{s \eta} \eta_{m}=\chi_{m} ; \\
& \mathrm{p} \eta_{m}=\mathrm{n}_{m}^{2} / \chi_{m} \\
& M_{s}=\left(\begin{array}{cc}
\operatorname{Cos}\left[\delta_{1}\right] & \mathrm{I} \frac{\operatorname{Sin}\left[\delta_{1}\right]}{\mathrm{s} \eta_{1}} \\
\mathrm{I} \operatorname{s} \eta_{1} \operatorname{Sin}\left[\delta_{1}\right] & \operatorname{Cos}\left[\delta_{1}\right]
\end{array}\right)\left(\begin{array}{cc}
\operatorname{Cos}\left[\delta_{2}\right] & \mathrm{I} \frac{\operatorname{Sin}\left[\delta_{2}\right]}{\mathrm{s} \eta_{2}} \\
\mathrm{I} \operatorname{s\eta } \eta_{2} \operatorname{Sin}\left[\delta_{2}\right] & \operatorname{Cos}\left[\delta_{2}\right]
\end{array}\right) \\
& \left(\begin{array}{ccc}
\operatorname{Cos}\left[\delta_{3}\right] & \mathrm{I} \operatorname{Sin}\left[\delta_{3}\right] / s \eta_{2} \\
\mathrm{I} \operatorname{s\eta } & \operatorname{Sin}\left[\delta_{3}\right] & \operatorname{Cos}\left[\delta_{3}\right]
\end{array}\right)\left(\begin{array}{cc}
\operatorname{Cos}\left[\delta_{4}\right] & \mathrm{I} \operatorname{Sin}\left[\delta_{4}\right] / s \eta_{2} \\
\mathrm{I} \mathrm{s} \eta_{2} \operatorname{Sin}\left[\delta_{4}\right] & \operatorname{Cos}\left[\delta_{4}\right]
\end{array}\right) ;
\end{aligned}
$$

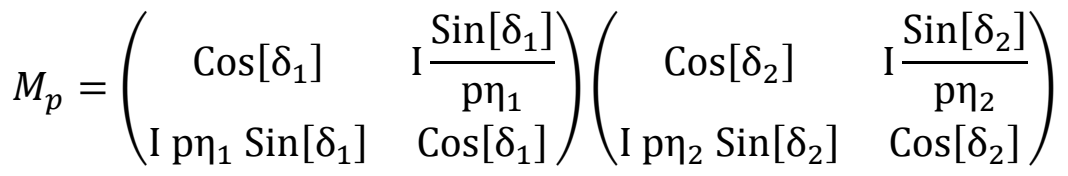

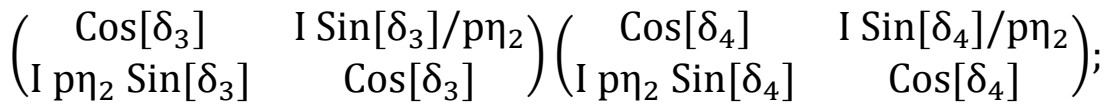

$$
\begin{aligned}
& V_{s}=\left(\begin{array}{c}
1 \\
s \eta_{m}
\end{array}\right) ; T_{s}=M_{s} V_{s} \text {; } \\
& V_{p}=\left(\begin{array}{c}
1 \\
\mathrm{p} \eta_{m}
\end{array}\right) \text {; } \\
& T_{p}=M_{p} V_{p} \text {; } \\
& B_{s}=T_{s}[1,1] ; C_{s}=T_{s}[2,1] ; \\
& B_{p}=T_{p}[1,1] ; C_{p}=T_{p}[2,1] ; \\
& R_{S}=\operatorname{Re}\left[\frac{s \eta_{0} B_{S}-C_{S}}{s \eta_{0} B_{S}+C_{s}} \text { conjugate }\left(\frac{s \eta_{0} B_{S}-C_{S}}{s \eta_{0} B_{S}+C_{S}}\right)\right] \text {; } \\
& T_{S}=\operatorname{Re}\left[\frac{4 \eta_{0} R e\left[s \eta_{m}\right]}{s \eta_{0} B_{S}+C_{S}} \text { conjugate }\left(\frac{1}{s \eta_{0} B_{S}+C_{S}}\right)\right] ; \\
& A_{s}=\operatorname{Re}\left[\frac{4 s \eta_{0} R e\left[B_{s} \text { conjugate }\left[C_{S}\right]-s \eta_{m}\right]}{s \eta_{0} B_{S}+C_{S}} \text { conjugate }\left(\frac{1}{s \eta_{0} B_{S}+C_{S}}\right)\right] ; \\
& R_{p}=\operatorname{Re}\left[\frac{\mathrm{p} \eta_{0} B_{p}-C_{p}}{\mathrm{p}_{0} B_{p}+C_{p}} \text { conjugate }\left(\frac{\mathrm{p} \eta_{0} B_{p}-C_{p}}{\mathrm{p} \eta_{0} B_{p}+C_{p}}\right)\right] ; \\
& T_{p}=\operatorname{Re}\left[\frac{4 \eta_{0} R e\left[\mathrm{p}_{m}\right]}{\mathrm{p} \eta_{0} B_{p}+C_{p}} \text { conjugate }\left(\frac{1}{\mathrm{p}_{0} B_{p}+C_{p}}\right)\right] ;
\end{aligned}
$$


$A_{p}=\operatorname{Re}\left[\frac{4 \mathrm{p} \eta_{0} R e\left[B_{p} \text { conjugate }\left[C_{p}\right]-\mathrm{s} \eta_{m}\right]}{\mathrm{p} \eta_{0} B_{p}+C_{p}}\right.$ conjugate $\left.\left(\frac{1}{\mathrm{p}_{0} B_{p}+C_{p}}\right)\right] ;$

Theta $[\mathrm{i}]=\theta$;

$R_{s}[\mathrm{i}]=R_{s} ; R_{p}[\mathrm{i}]=R_{p} ;$

$\operatorname{Print}[\theta]$;

$\operatorname{Print}\left[\frac{4 \mathrm{n}_{m} \mathrm{n}_{0}}{\left(\mathrm{n}_{m}+\mathrm{n}_{0}\right)^{2}} T_{s}\right]$

$\mathrm{i}+=1$;

If $[\mathrm{i}<(\mathrm{M}+1)$, Goto[aa]];

saf0=Table[\{Theta[i], $\left.\left.R_{S}[\mathrm{i}], R_{p}[\mathrm{i}]\right\},\{\mathrm{i}, 0, \mathrm{M}, 1\}\right]$;

Export["saf0.xls",saf0]; 


\section{APPENDIX 10: Permissions}

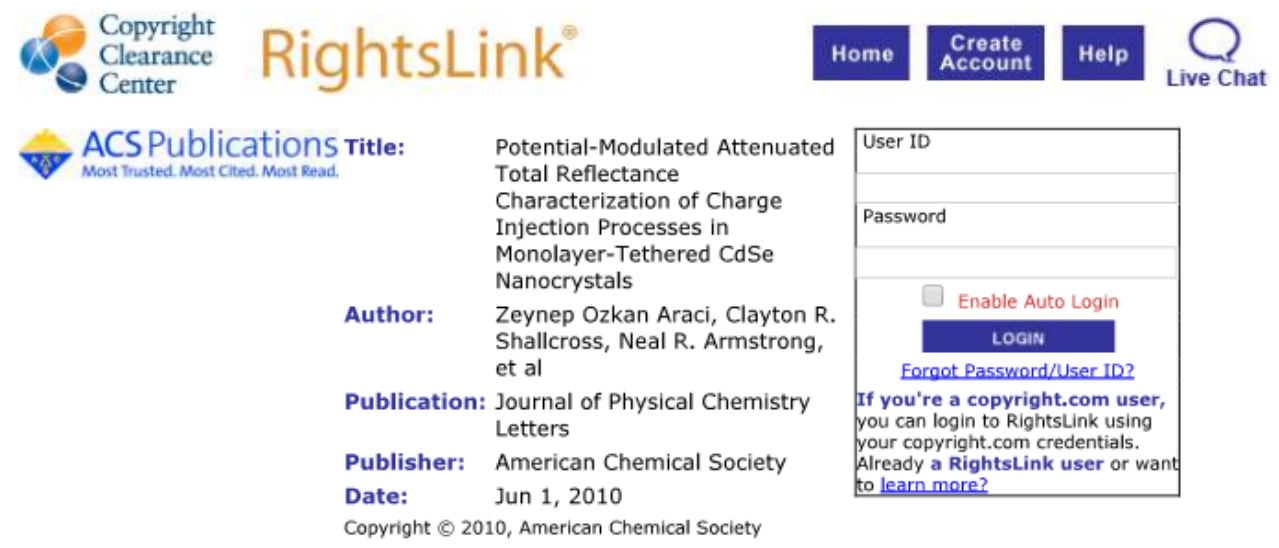

PERMISSION/LICENSE IS GRANTED FOR YOUR ORDER AT NO CHARGE

This type of permission/license, instead of the standard Terms \& Conditions, is sent to you because no fee is being charged for your order. Please note the following:

- Permission is granted for your request in both print and electronic formats, and translations.

- If figures and/or tables were requested, they may be adapted or used in part.

- Please print this page for your records and send a copy of it to your publisher/graduate school.

- Appropriate credit for the requested material should be given as follows: "Reprinted (adapted) with permission from (COMPLETE REFERENCE CITATION). Copyright (YEAR) American Chemical Society." Insert appropriate information in place of the capitalized words.

- One-time permission is granted only for the use specified in your request. No additional uses are granted (such as derivative works or other editions). For any other uses, please submit a new request.

If credit is given to another source for the material you requested, permission must be obtained from that source.

\section{BACK CLOSE WINDOW}

Copyright (c) 2014 Copyright Clearance Center. Inc. All Rights Reserved. Privacy statement Comments? We would like to hear from you. E-mail us at customercare@copyriaht.com 


Copyright $\begin{aligned} & \text { Clearance } \\ & \text { Center }\end{aligned}$
$\begin{array}{ll}\text { ACSPublications Title: } & \begin{array}{l}\text { Angle-Multiplexed Waveguide } \\ \text { Resonance of High Sensitivity } \\ \text { and Its Application to } \\ \text { Nansted. Mostcited. Most Read. } \\ \text { Molecular Assemblies }\end{array} \\ \text { Publication: } & \begin{array}{l}\text { Courtney L. Byard, Xue Han, } \\ \text { Sergio B. Mendes }\end{array} \\ \text { Publisher: } & \text { American Chemical Society } \\ \text { Date: } & \text { Nov } 1,2012 \\ \text { Copyright (c) 2012, American Chemical Society }\end{array}$

\begin{tabular}{|c|}
\hline User ID \\
\hline Password \\
\hline Enable Auto Login \\
\hline Locin \\
Eorgot Password/User ID? \\
If you're a copyright.com user, \\
you can login to RightsLink using \\
your copyright.com credentials. \\
Already a RightsLink user or want \\
to learn more?
\end{tabular}

\section{PERMISSION/LICENSE IS GRANTED FOR YOUR ORDER AT NO CHARGE}

This type of permission/license, instead of the standard Terms \& Conditions, is sent to you because no fee is being charged for your order. Please note the following:

- Permission is granted for your request in both print and electronic formats, and translations.

- If figures and/or tables were requested, they may be adapted or used in part.

- Please print this page for your records and send a copy of it to your publisher/graduate school.

- Appropriate credit for the requested material should be given as follows: "Reprinted (adapted) with permission from (COMPLETE REFERENCE CITATION). Copyright (YEAR) American Chemical Society." Insert appropriate information in place of the capitalized words.

- One-time permission is granted only for the use specified in your request. No additional uses are granted (such as derivative works or other editions). For any other uses, please submit a new request.

\section{BACK}

CLOSE WINDOW 


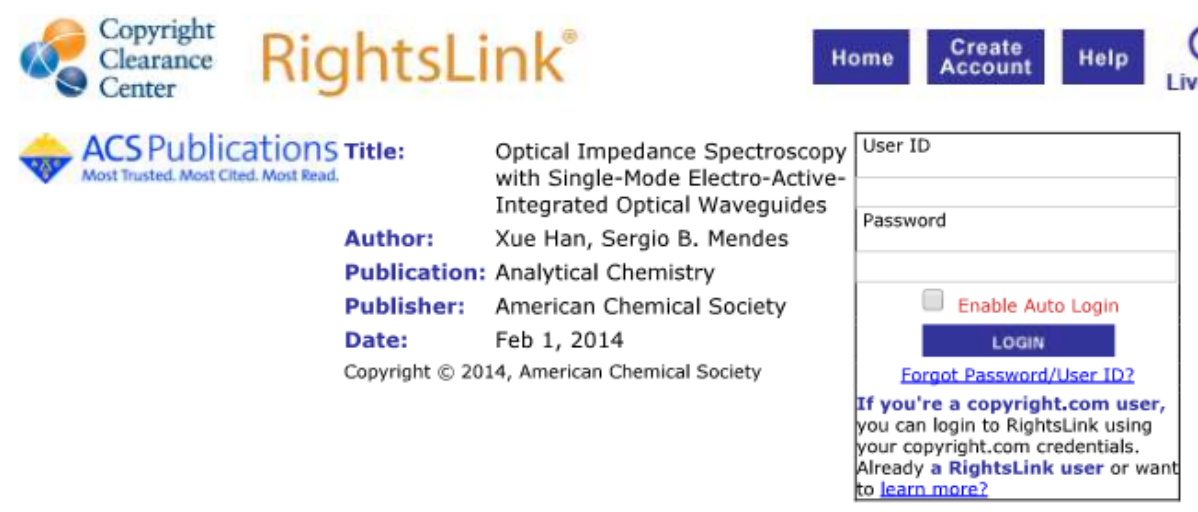

\section{PERMISSION/LICENSE IS GRANTED FOR YOUR ORDER AT NO CHARGE}

This type of permission/license, instead of the standard Terms \& Conditions, is sent to you because no fee is being charged for your order. Please note the following:

- Permission is granted for your request in both print and electronic formats, and translations.

- If figures and/or tables were requested, they may be adapted or used in part.

- Please print this page for your records and send a copy of it to your publisher/graduate school.

- Appropriate credit for the requested material should be given as follows: "Reprinted (adapted) with permission from (COMPLETE REFERENCE CITATION). Copyright (YEAR) American Chemical Society." Insert appropriate information in place of the capitalized words.

- One-time permission is granted only for the use specified in your request. No additional uses are granted (such as derivative works or other editions). For any other uses, please submit a new request.

\section{BACK}

CLOSE WINDOW

Copyright (c) 2014 Copyright Clearance Center. Inc. All Rights Reserved. Privacy statement. Comments? We would like to hear from you. E-mail us at customercare@copyright.com 


\title{
CURRICULUM VITAE
}

\author{
Xue Han, PhD
}

xuehan0mia@gmail.com

EDUCATION

- $\quad$ Master of Science (Physics), University of Louisville, GPA 3.925/4, May 2014

- $\quad$ Master of Science (Optics), Dalian University of Technology (China), GPA 3.64/4, July 2009

- Bachelor of Science (Optical Information of Science and Technology), Dalian University of Technology (China), Rank 14/150, July 2006

PROFESSIONAL RESEARCH EXPERIENCE

- $\quad$ Research Assistant in Photonics Lab, 2009 - 2014, University of Louisville

- $\quad$ Research Assistant in Institute of Near-Field Optics \& Nano Technology, 2007 - 2009, Dalian University of Technology

\section{GRANTS AND AWARDS}

- $\quad$ The Graduate Dean's Citation, presented to no more than $10 \%$ of a program's graduates, University of Louisville, 2014

- $\quad$ Dissertation completion award, University of Louisville, 2014 
- $\quad$ Sponsored research tuition award, University of Louisville, 2013

- $\quad$ Travel grant, The Optical Society, Advanced Photonics Congress, July 2013, Puerto Rico

- $\quad$ Sponsored research tuition award, University of Louisville, 2010

- $\quad$ Outstanding Undergraduate Student Award, Dalian University of Technology, China, 2006

- $\quad$ Third Grade Comprehensive Scholarship, Dalian University of Technology, China, 2006

- Third Grade Comprehensive Scholarship, Dalian University of Technology, China, 2005

- $\quad$ Third Grade Comprehensive Scholarship, Dalian University of Technology, China, 2004

- Scholarship of Individual Event, Dalian University of Technology, China, 2003 PUBLICATIONS

- $\quad$ Optical Impedance Spectroscopy with Single-Mode Electro-Active Integrated Optical Waveguides. Xue Han, and Sergio B. Mendes. Analytical Chemistry, DOI:10.1021/ac4030736.

- Studies of Redox Reactions in Electro-Active Proteins using Optical Impedance Spectroscopy at Single-Mode Waveguides. Xue Han and Sergio B. Mendes. Proc. of SPIE Vol. 8812 881207-1; DOI: 10.1117/12.2024106; 2013

- $\quad$ Spectroscopic Studies on Ultra-Thin Films of Indium Tin Oxide under ElectroChemical Modulation. Han Xue ; Sergio B. Mendes . Proc. of SPIE Vol. 8459 
845911-1; DOI:10.1117/12.2000142; 2012

- Angle-Multiplexed Waveguide Resonance of High Sensitivity and Its Application to Nanosecond Dynamics of Molecular Assemblies. Courtney L. Byard, Xue Han , and Sergio B. Mendes. Analytical chemistry, 2012, 84 (22), pp 9762-9767; DOI: 10.1021/ac302812u

- $\quad$ Force Calculation of Cells Pushed by Patch Clamp micropipette Using Optical Tweezers. The First National Conference on the Application of Optical Tweezers, 2008, volume 1, pp 23.

\section{PRESENTATIONS}

- $\quad$ The Optical Society, Optical Sensors, SeTh 3Bb.3, July 2014, Spain

- $\quad$ American Chemical Society, Chemistry in Motion, ANYL 237, September 2013, Indianapolis, Indiana

- $\quad$ Kentucky Nano-Symposium, August 2013, Louisville, Kentucky

- $\quad$ SPIE, Optics and Photonics, 8812-6, August 2013, San Diego, California

- $\quad$ The Optical Society, Advanced Photonics Congress, JT3A.34, July 2013, Puerto Rico

- Electron, Ion, and Photon Beam Technology and Nanofabrication, P13-03, May 2013, Nashville, Tennessee

- $\quad$ SPIE, Optics and Photonics, 8459-38, August 2012, San Diego, California

- $\quad$ 17th Annual KY EPSCoR Conference, May 2012, Lexington, Kentucky

- $\quad$ 16th Annual KY EPSCoR Conference, May 2011, Lexington, Kentucky

- $\quad$ 15th Annual KY EPSCoR Conference, May 2010, Lexington, Kentucky 